\title{
Design of an Optical Fiber-Coupled Sensor for Ambient Methane Measurement
}

by

\section{Stephen Barry Schoonbaert}

\begin{abstract}
A thesis submitted to the Faculty of Graduate and Postdoctoral Affairs in partial fulfillment of the requirements for the degree of
\end{abstract}

\section{Doctor of Philosophy}

In

\section{Mechanical Engineering}

Ottawa-Carleton Institute of Mechanical and Aerospace Engineering Carleton University

Ottawa, Ontario

(C) 2015

Stephen Barry Schoonbaert 


\section{Abstract}

A tunable diode laser system was developed for remote monitoring of ambient methane using fiber optically connected all-optical sensor heads. Experiments were performed to quantitatively evaluate the influence of selected system hardware and software configurations, with the further goal of enabling measurements of ambient methane concentrations over an intrinsically-safe fiber optic network at sufficient precision and sensitivity to detect unknown fugitive emission sources. The designed system could switch between the sweep integration (SI) and wavelength modulation spectroscopy (WMS) detection methods, which inferred the methane volume mixing ratio from an absorption or second-harmonic (2f) feature respectively. Signals controlling the laser injection current were optimized to balance trade-offs between measurement precision and system sensitivity, and fiber optic components were thermally stabilized to reduce system drift. Starting from this base system, experiments were performed to evaluate the effectiveness of theory-based and experimental calibration methods, software and dual laser approaches to estimating the absorption-free intensity, different signal processing approaches to suppress effects of residual amplitude modulation (RAM) of the laser output intensity, and methods to reduce system drift. Tests also considered effects of varying fiber lengths between the central laser control hardware and the remotely located optical sensor heads. Finally, long-term stability was evaluated by quantifying bias (drift) and precision uncertainty in tests up to 16 months after initial calibration. The best measurement performance was achieved using the WMS method with thermally stabilized optical components within the central control hardware combined with a theory-based calibration, automated daily calibration supported by concurrent softwarebased estimation of the absorption-free intensity, and pair-wise averaging of the $2 f$ feature maxima in each sweep period to suppress effects of RAM. At an averaging time of $1 \mathrm{~s}$ and methane concentrations between 1.0 and $50 \mathrm{ppm}_{\mathrm{v}}$, the system implementing the $2 f$-WMS method surpassed the methane volume mixing ratio measurement targets with a precision of $1.6 \mathrm{ppm}_{\mathrm{v}}$ (absorbance of $6.57 \times 10^{-7} \mathrm{~cm}^{-1}$ ) and lower detection limit (LDL) of $1.5 \mathrm{ppm}_{\mathrm{v}}\left(6.13 \times 10^{-7} \mathrm{~cm}^{-1}\right)$. In achieving these values, thermal stabilization was responsible for a 93\% reduction in the precision uncertainty; adding the concurrent software-based absorption-free intensity estimate netted a further reduction of $73 \%$; and implementing RAM suppression via pair-wise averaging of the resolved $2 f$ features within each sweep period technique allowed a further reduction of $9 \%$. 


\section{Acknowledgements}

I would like to sincerely thank my supervisor Matthew Johnson for the guidance, support, and patience he gave me over the course of my graduate studies. I am fortunate to have worked with such a dedicated teacher and engineer.

Also, I would like to thank Jacques Albert for sharing his time, knowledge, and insight during our impromptu meetings. His indispensable advice and guidance helped me see problems from a different perspective and identify solutions which were not immediately apparent.

I would also like to thank David Tyner for his advice and technical support over the past few years. His patience, dedication, and positive attitude made daunting tasks seem possible. I am a better person to have worked with him.

I would also like to thank my parents and siblings for their unwavering support and encouragement which helped me persevere through trying times. Thank you.

Finally, I would like to thank my wife Heidi for her immeasurable and continuing support and patience. Her abundance of love and encouragement continues to amaze me.

Thank you. 


\section{Table of Contents}

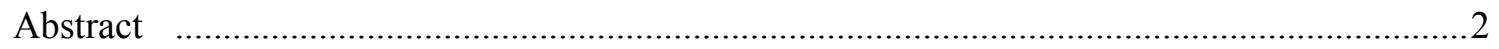

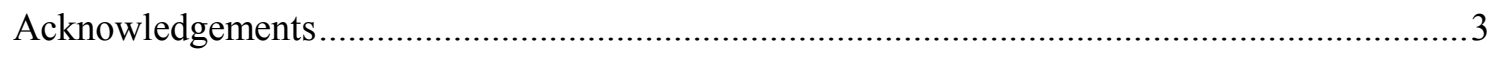

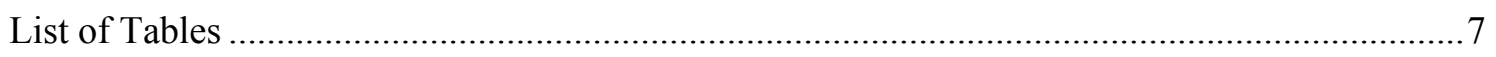

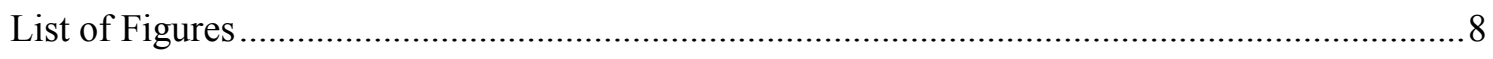

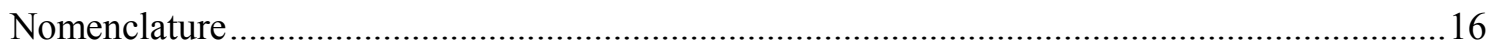

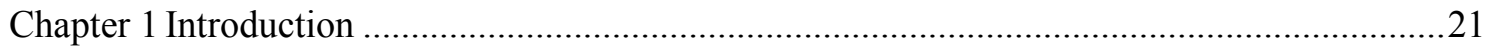

1.1 Motivation - Fugitive Emission Detection in Gas-Processing plants .........................21

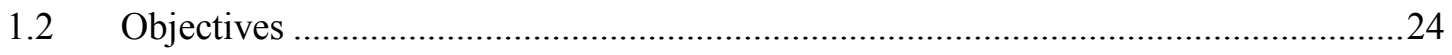

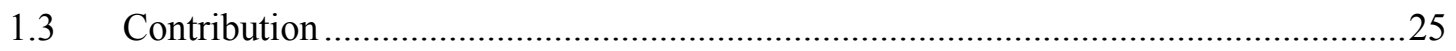

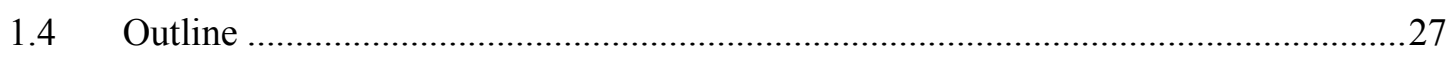

Chapter 2 Background and Absorption Spectroscopy Theory .................................................28

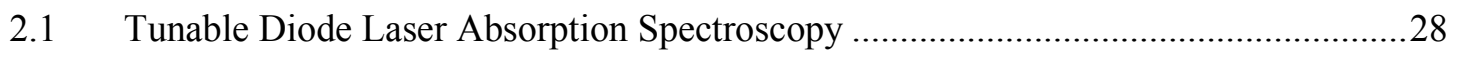

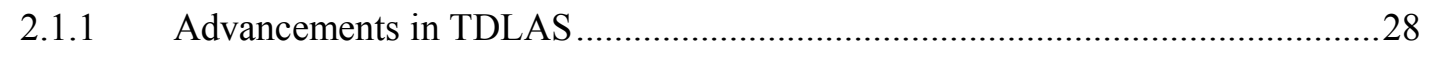

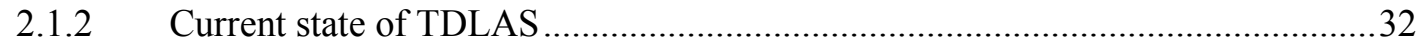

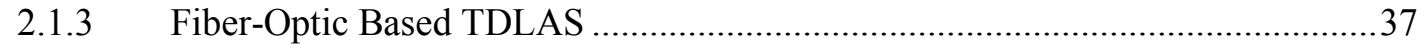

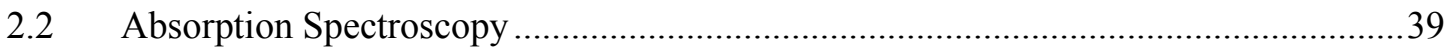

2.2.1 Laser Driving Signals and Associated Spectroscopic Techniques.......................43

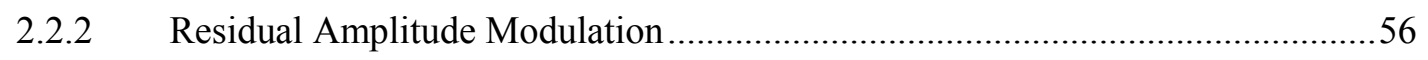

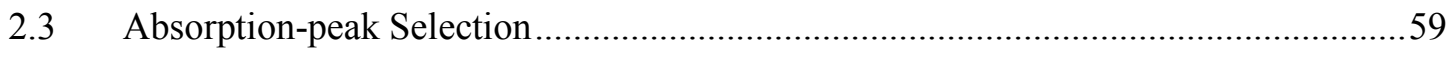

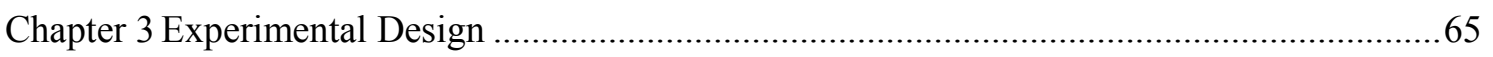

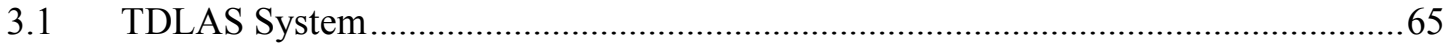

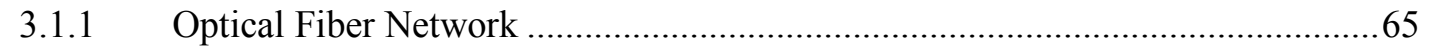

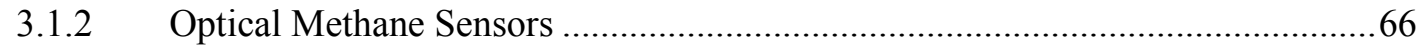

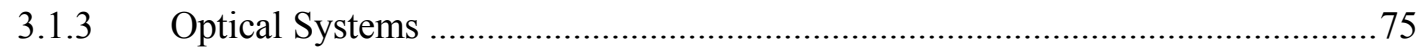

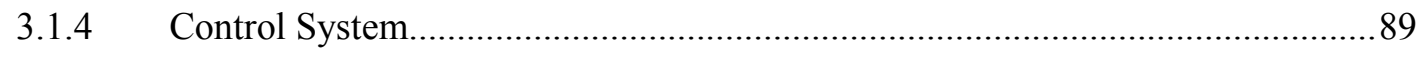

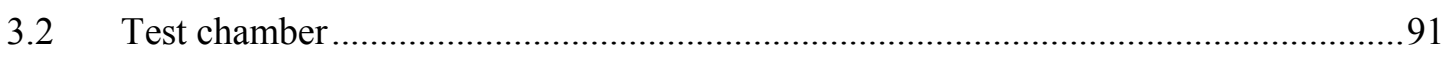

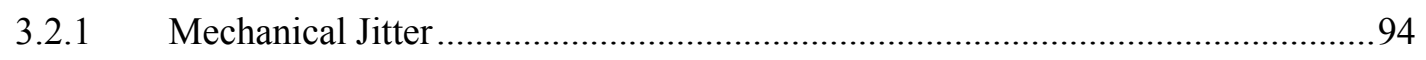

Chapter 4 Signal Processing and Control........................................................................ 98

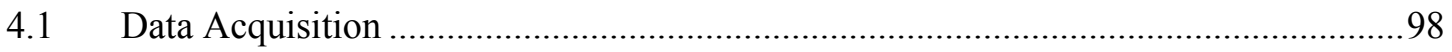

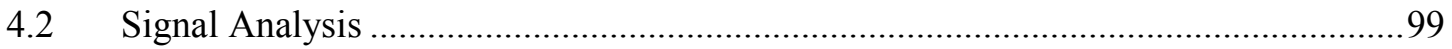




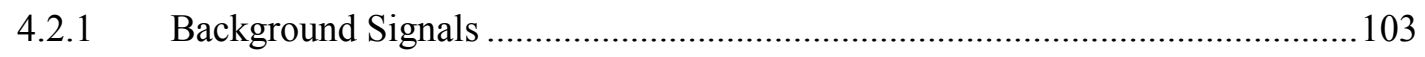

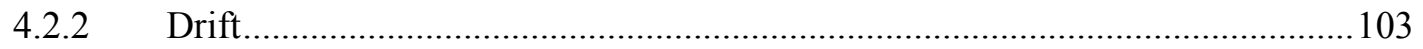

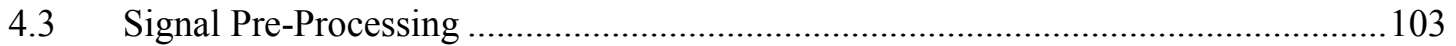

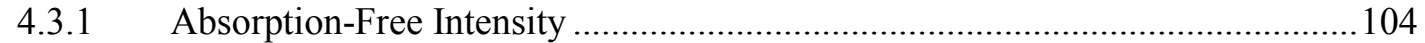

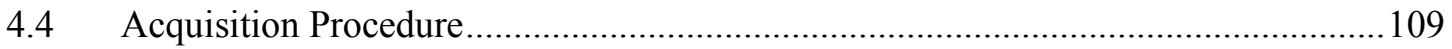

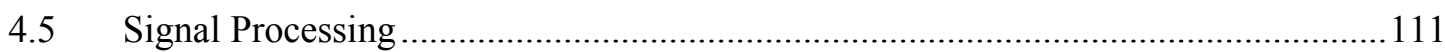

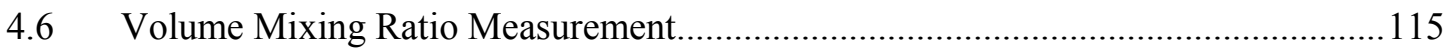

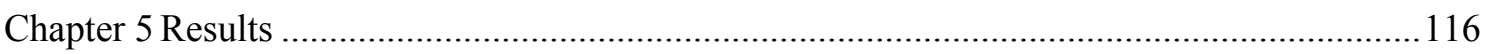

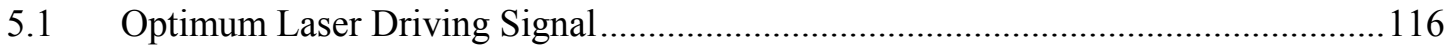

5.1.1 Optimum Laser Driving Sweep Frequency and Amplitude for SI Method ........118

5.1.2 Optimum WMS Laser Driving Signal Frequencies and Amplitudes..................120

5.2 Software Analysis Algorithms to Maximize Measurement Precision ........................ 124

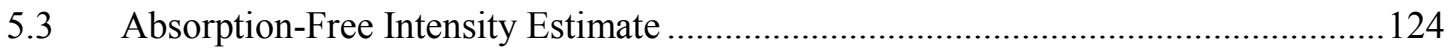

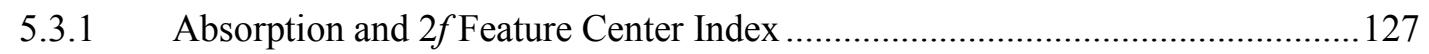

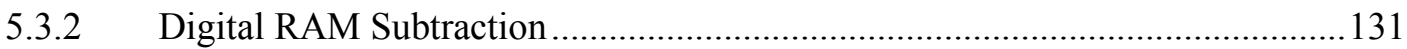

5.4 Hardware Configurations to Maximize Measurement Precision ................................ 135

5.4.1 Mitigation of Polarization-Dependant Intensity Drift....................................... 135

5.4.2 Effects of Fiber-Length on Measurement Accuracy ….....................................138

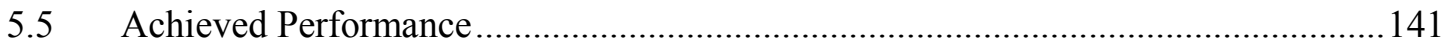

5.5.1 Quantified Precision and Lower Detection Limits........................................... 141

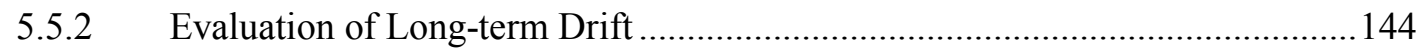

5.5.3 Final Results and Comparison to Literature....................................................... 147

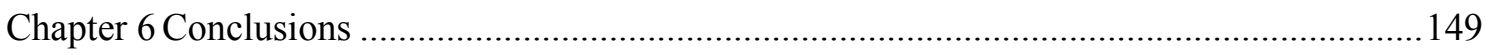

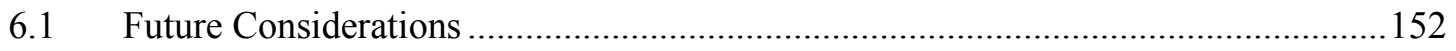

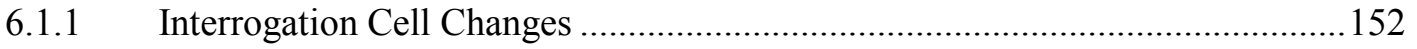

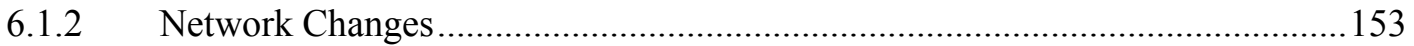

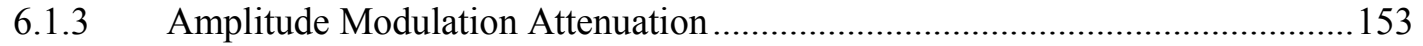

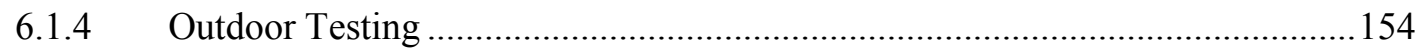

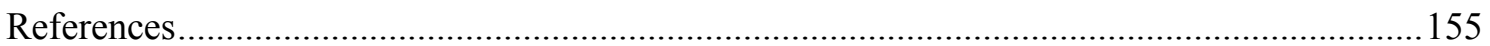

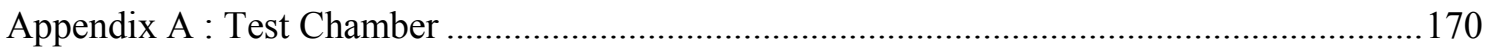

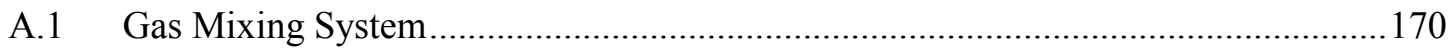

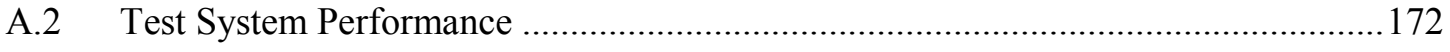

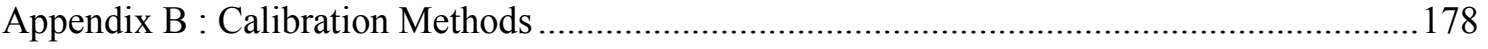




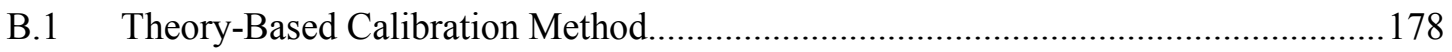

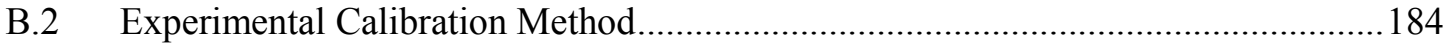

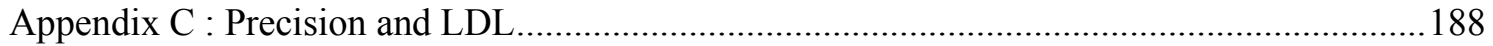

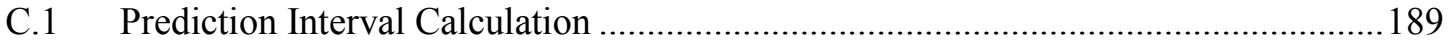

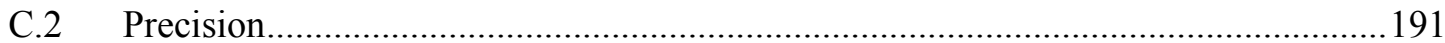

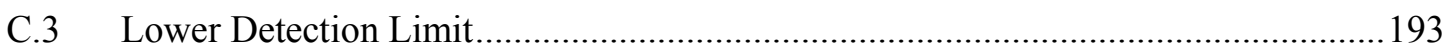




\section{List of Tables}

Table 2.1a: Details and Performance Characteristics of Current TDLAS Systems ....................... 33

Table 2.2: Performance Characteristics of Fiber-Based TDLAS Systems ................................... 38

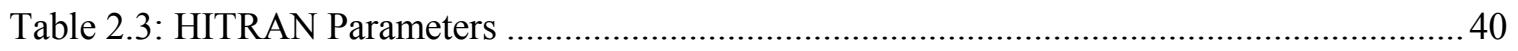

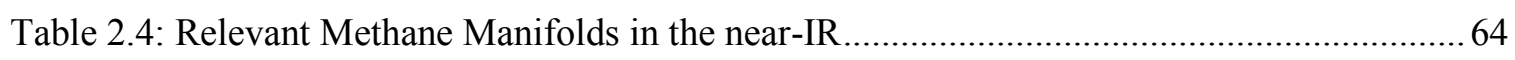

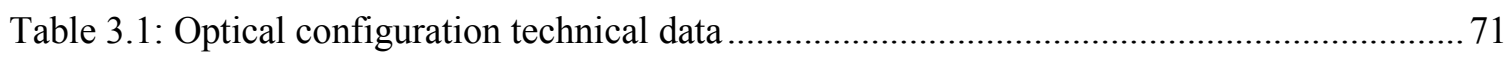

Table 3.2: TDLAS System Detector Specifications .................................................................. 79

Table 3.3: Laser Diode Output Characteristics ........................................................................ 83

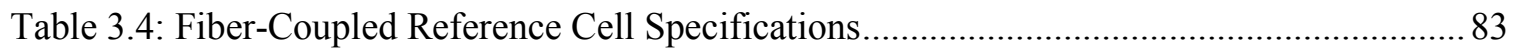

Table 3.5: Analog Signal Digitization Specifications............................................................... 90

Table 3.6: Atmospheric Sensing Component Specifications ..................................................... 91

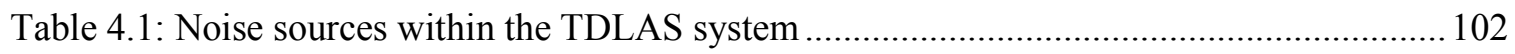

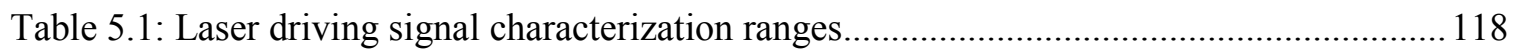

Table 5.2: Optimum laser driving signal amplitudes and frequencies for the SI and WMS

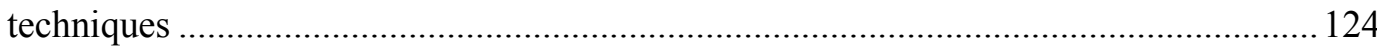

Table 5.3: SI measurement precision at 2 and $50 \mathrm{ppm}_{\mathrm{v}}$ for various calibration and absorption-free

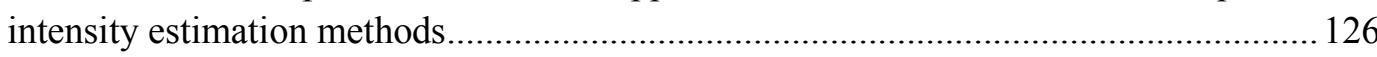

Table 5.4: WMS measurement precision at 2 and $50 \mathrm{ppm}_{\mathrm{v}}$ for various calibration and absorption-

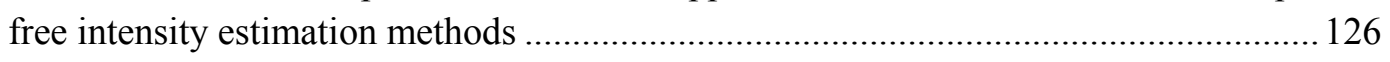

Table 5.5: Methane measurement performance of the baseline SI and WMS system configurations compared to the required performance

Table 5.6: Performance Characteristics of Fiber-Based TDLAS Systems Including Measurement

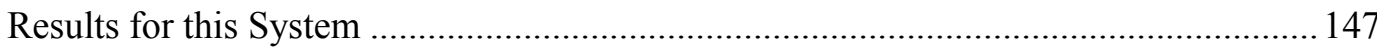

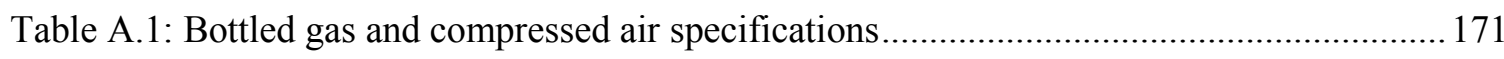

Table A.2: Mass-flow controller specifications .................................................................... 171

Table A.3: Fourier Transform Infrared Spectroscopy System Specifications ............................. 172

Table B.1: Look-up table parameters and resolution .......................................................... 179 


\section{List of Figures}

Figure 1.1: Total emissions by activity in Canada's Oil and Gas Sector. Data obtained from the 1990-2013 National Inventory Report (Environment Canada, 2014), which assumes a

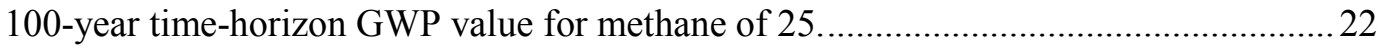

Figure 2.1: System schematic illustrating a low-frequency sweep injection current resulting in a low-frequency intensity and wavelength output.

Figure 2.2: Measured intensity (red) and wavelength (green) SI signals resulting from an injection current sweep (blue).

Figure 2.3: Simulated intensity (Red) and wavenumber (green) SI signal components and the corresponding laser characteristics (derived from experimental results).

Figure 2.4: Simulated absorption-free return signal (black) and simulated and experimental (1-second average) SI intensity signals after absorption due to $200 \mathrm{ppm}_{\mathrm{v}}$ methane in nitrogen. Simulated signals were calculated at the experimental conditions (at ambient pressures and temperatures, and an optical path length of $5.79 \mathrm{~m}$ ), with $\boldsymbol{v}=1653.73 \mathrm{~nm}$ $\left(6046.94 \mathrm{~cm}^{-1}\right), a_{s} \approx 0.015 \mathrm{~nm}\left(\sim 0.009 \mathrm{~cm}^{-1}\right)$.

Figure 2.5: Simulated and experimental (1-second average) SI absorption signals with $200 \mathrm{ppm}_{\mathrm{v}}$ methane in nitrogen. Simulated signals were calculated at the experimental conditions (at ambient pressures and temperatures, and an optical path length of $5.79 \mathrm{~m})$, with $\boldsymbol{v}=1653.73 \mathrm{~nm}\left(6046.94 \mathrm{~cm}^{-1}\right), a_{s} \approx 0.015 \mathrm{~nm}\left(\sim 0.009 \mathrm{~cm}^{-1}\right)$.

Figure 2.6: System schematic illustrating a summed low-frequency 'sweep' and high-frequency laser diode injection current modulation, resulting in a dual-frequency modulated output

Figure 2.7: Raw WMS intensity absorption-free intensity (red) and wavelength (green) signals resulting from an injection current modulation (blue)

Figure 2.8: Simulated WMS characterization signals for the low- (a) and high-frequency

(b) simulated absorption-free intensity (Red) and wavenumber (green), highlighting the corresponding laser characteristics

Figure 2.9: Simulated absorption-free intensity (black), and simulated (grey) and experimental (red) WMS intensity signals generated with $200 \mathrm{ppm}_{\mathrm{v}}$ methane in nitrogen, calculated using an optical path-length of $5.79 \mathrm{~m}$, temperature of $23^{\circ} \mathrm{C}$, and pressure of $101.325 \mathrm{kPa}$, with $\boldsymbol{v}=1653.73 \mathrm{~nm}\left(6046.94 \mathrm{~cm}^{-1}\right), a_{s} \approx 0.015 \mathrm{~nm}\left(\sim 0.009 \mathrm{~cm}^{-1}\right)$, and, $a_{m} \approx 0.032 \mathrm{~nm}\left(\sim 0.020 \mathrm{~cm}^{-1}\right)$.

Figure 2.10: Simulated and experimental (1-second average) WMS $2 f$ signals with $200 \mathrm{ppm}_{\mathrm{v}}$ methane in nitrogen. Simulated signals were calculated at the experimental conditions (at ambient pressures and temperatures, and an optical path length of $5.79 \mathrm{~m})$, with $\boldsymbol{v}$ $=1653.73 \mathrm{~nm}\left(6046.94 \mathrm{~cm}^{-1}\right), a_{s} \approx 0.015 \mathrm{~nm}\left(\sim 0.009 \mathrm{~cm}^{-1}\right)$, and,$a_{m} \approx 0.032 \mathrm{~nm}$ $\left(\sim 0.020 \mathrm{~cm}^{-1}\right)$. 
Figure 2.11: Simulated WMS $2 f$ Signals and Residuals with and without RAM Subtraction: (a) $2 \mathrm{ppm}_{\mathrm{v}}$, (b) $200 \mathrm{ppm}_{\mathrm{v}}$ with an optical path-length of $5.79 \mathrm{~m}$, at $23^{\circ} \mathrm{C}$ and $101.325 \mathrm{kPa}$.

Figure 2.12: Percent-difference of simulated $2 f$ values obtained at the $2 v_{3} \mathrm{R}(3)$ methane absorption manifold with and without RAM-subtraction, evaluated against the ideal WMS signal without RAM (no sweep) using an optical path-length of $5.79 \mathrm{~m}$, at $23^{\circ} \mathrm{C}$ and $101.325 \mathrm{kPa}$.

Figure 2.13: Percent-difference of simulated methane volume mixing ratio at the $2 v_{3} R(3)$ methane manifold with and without RAM-subtraction, evaluated against the ideal WMS signal without RAM (no sweep) using an optical path-length of $5.79 \mathrm{~m}$, at $23^{\circ} \mathrm{C}$ and $101.325 \mathrm{kPa}$.

Figure 2.14: Comparison of methane spectral absorption bands (grey shaded regions) to standard 9/125 $\mu \mathrm{m}$ telecommunications fiber optic attenuation and semiconductor detector responsivity in the Near-IR. Adapted from (Schubert, 2003; Roberson and Cook, 2011).

Figure 2.15: Spectral lines of significant atmospheric absorbers within this spectral region. Data from HITRAN2012 (Rothman et al., 2013).

Figure 2.16: Absorption strength of near-IR $2 v_{3} \mathrm{Q}$ and $\mathrm{R}$ methane absorption bands; including prominent atmospheric absorbers for reference. Calculated using a Lorentz profile at $20^{\circ} \mathrm{C}$ and $101.325 \mathrm{kPa}$, using a $5.79 \mathrm{~m}$ optical path-length.

Figure 2.17: Combined absorption of all significant standard atmospheric absorbers (i.e. $1.7 \mathrm{ppm}_{\mathrm{v}}$ $\mathrm{CH}_{4}, 15,000 \mathrm{ppm}_{\mathrm{v}} \mathrm{H}_{2} \mathrm{O}, 365 \mathrm{ppm}_{\mathrm{v}} \mathrm{CO}_{2}, 0.32 \mathrm{ppm}_{\mathrm{v}} \mathrm{N}_{2} \mathrm{O}$, and $0.1 \mathrm{ppm}_{\mathrm{v}} \mathrm{CO}$ ) around the $\mathrm{R}(3), \mathrm{R}(4)$, and $\mathrm{Q}(6)$ methane manifolds. Plots were calculated at $20^{\circ} \mathrm{C}$ and $101.325 \mathrm{kPa}$, using a $5.79 \mathrm{~m}$ optical path-length.

Figure 3.1: Fiber-optic multiplexing techniques; space-, frequency-, and wavelength-division multiplexing

Figure 3.2: Generic optical routing configurations in and out of an interrogation region. a) straight through; b) fiber-in, detector out; c) dual fiber-in/-out; d) single fiber-in/-out.

Figure 3.3: Lens collimators tested for use in the optical sensor. a) custom $\varnothing 4.0 \mathrm{~mm}$ beam, b) small $\varnothing 1.6 \mathrm{~mm}$ beam, c) doublet collimator $Ø 7.0 \mathrm{~mm}$ beam, micro laser systems FC20 $\varnothing 10.6 \mathrm{~mm}$ beam

Figure 3.4: Component configurations used to test the influence of optical path length and components on return intensity and absorption signal strength .70

Figure 3.5: Effects of methane volume mixing ratio and optical path length on SI (absorption) and WMS (second-harmonic, $2 f$ ) feature SNR, and return intensity. The SI results were obtained at a sweep frequency of $300 \mathrm{~Hz}$, while the WMS results were obtained using a sweep frequency of $100 \mathrm{~Hz}$ and a modulation frequency of $20 \mathrm{kHz}$. Data was acquired at the $2 v_{3} \mathrm{R}(3)$ methane absorption manifold, ambient pressures and temperatures, and with the various optical sensor heads coupled to the base station with a $6-\mathrm{m} 9 / 125 \mu \mathrm{m}$ fiber optic cable.

Figure 3.6: Optical fiber-coupled 5.79 meter open-path multipass optical sensor head prototype; demonstrated here with the removable test chamber support structures on the ends ........74 
Figure 3.7: Illustration of the laser beam path though the fiber coupled optical sensor head........ 74

Figure 3.8: Optical sub-system schematic; (A) 2-by-2 switch and controller, (B) AVOA, (C) taps, laser reference $(\mathrm{C} 1)$, reference cell $(\mathrm{C} 2)$, FRR $(\mathrm{C} 3)$, and multiplexing $(\mathrm{C} 4)$ routes ......... 76

Figure 3.9: Thermally stabilized enclosure exterior and cross-section (A-A) ..............................78

Figure 3.10: InGaAs and extended InGaAs detector responsivity curves. Adapted from (Laser Components, 2015a; Laser Components, 2015b)

Figure 3.11: Simulated SI (a) and WMS (b) methane measurements, including the $95 \%(2 \sigma)$ measurement precision for a signal averaged for 10 seconds. Computed at the $2 v_{3} \mathrm{R}(3)$ absorption manifold, at $23^{\circ} \mathrm{C}$ and $101.325 \mathrm{kPa}$, with a $5.79 \mathrm{~m}$ optical path-length, and experimentally-obtained laser and noise intensity characteristics ................................. 81

Figure 3.12: Thermal spectral tuning ranges of Laser 1 and Laser 2 ......................................... 82

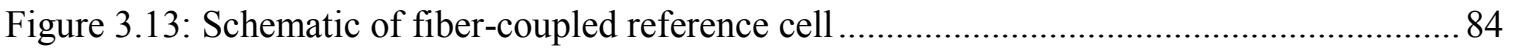

Figure 3.14: Inferred remote optical cell $2 f$ value from reference cell $2 f$ signal maximum........... 84

Figure 3.15: Extremes of the simulated transmittance through the fiber-coupled reference cell for the methane $2 v_{3} \mathrm{R}(3)$ absorption manifold, calculated using Lorentzian profiles and the $95 \%$ uncertainties from the reference cell specifications ( $p=1$ bar \pm 0.05 bar, methane volume mixing ratio $=55,000 \mathrm{ppm}_{\mathrm{v}} \pm 5,500 \mathrm{ppm}_{\mathrm{v}} \mathrm{CH}_{4}$ in $\mathrm{N}_{2}$, and path-length $=16.0 \mathrm{~mm}$ $\pm 1.0 \mathrm{~mm})$

Figure 3.16: Schematic of the fiber-ring resonator (FRR), with an input signal of varying intensity and wavelength, and the resultant output interference signal 86

Figure 3.17: Raw FRR calibration signals computed using the $2 v_{3}$ Q-branch for the methane reference cell containing $5.5 \% \mathrm{CH}_{4}$ in $\mathrm{N}_{2}$ at a pressure of 1.0 bar and an optical pathlength of $16 \mathrm{~cm}$.

Figure 3.18: Best-fit of the simulated and experimental reference cell absorption signals for the $\mathrm{Q}(3)-\mathrm{Q}(6)$ (left to right) methane absorption manifolds within the near-IR $2 v_{3}$ branch. The reference cell contained $5.5 \% \mathrm{CH}_{4}$ in $\mathrm{N}_{2}$ at a pressure of 1 bar and an optical pathlength of $16 \mathrm{~cm}$

Figure 3.19: FRR calibration signals; linearized FRR signal (grey), and absorption signal (red) from the reference cell containing $5.5 \% \mathrm{CH}_{4}$ in $\mathrm{N}_{2}$ at a pressure of 1.0 bar and an optical path-length of $16 \mathrm{~cm}$.

Figure 3.20: Control system routing diagram. LF and HF denote the low-frequency sweep and the high-frequency modulation signal generators respectively.

Figure 3.21: Test chamber cross-section.

Figure 3.22: Illustrated cross-section of the test chamber injection section ....

Figure 3.23: Test-section configurations; a) $5.79 \mathrm{~m}$ remote optical sensor, and b) component configuration test board. Note the flow direction and location of the thermistor

Figure 3.24: Fan location relative to exhaust end of test chamber. Note the location of the fan on the plywood support structure and the direction of the airflow, away from the test chamber. 
Figure 3.25: Absorption and $2 f$ value standard $1 \sigma$ deviation (a) at the $2 v_{3} \mathrm{R}(3)$ methane manifold peak with (red) and without (blue) the computer fan, and (b) the difference between the deviation with and without the fan. Data acquired using a $6 \mathrm{~m}$ optical fiber between the base station and $5.79 \mathrm{~m}$ sensor head and at ambient pressures and temperatures. 96

Figure 4.1: Effect of sample rate on measured interrogation cell absorption and $2 f$ values at the optimum SI and WMS laser modulation signals. Data acquired at ambient pressures and temperatures with the $5.79 \mathrm{~m}$ multipass sensor head.

Figure 4.2: Fast Fourier transform for 10 seconds of SI laser reference, reference cell, and interrogation cell signals highlighting the measured SI signals at integer multiples of 300 Hz. Signals were acquired at a test chamber methane volume mixing ratio of $100 \mathrm{ppm}_{\mathrm{v}}$ at ambient temperatures and pressures using the $5.79 \mathrm{~m}$ multipass sensor head coupled to

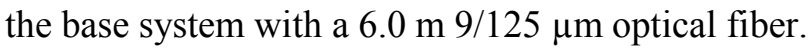

Figure 4.3: Fast Fourier transform for 10 seconds of WMS laser reference, reference cell, and interrogation cell signals highlighting the measured second-harmonic ( $2 f$ ) WMS signals for the combined modulation and sweep frequencies of $20 \mathrm{kHz}$ and $100 \mathrm{~Hz}$ respectively. Signals were acquired at a test chamber methane volume mixing ratio of $100 \mathrm{ppm}_{\mathrm{v}}$ at ambient temperatures and pressures using the 5.79 m multipass sensor head coupled to

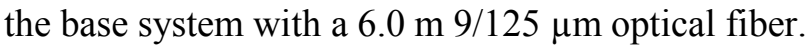

Figure 4.4: Linear estimates of the absorption-free temperature dependant laser characteristics responding to an input-current sweep signal of $100 \mathrm{~Hz}$ with an amplitude of $0.075 \mathrm{~V}$ $(\sim 1.5 \mathrm{~mA})$. Mean sweep intensity is denoted using circles, the first- and second-order harmonic characteristics (intensity amplitude and wavelength/intensity phase-shift) are denoted by squares and triangles respectively.

Figure 4.5: Laser output drift over 5700 separate data-acquisition 'runs'. Plotted is the resultant mean intensity for the combined diode temperature and injection current offset to stay centered on the $2 v_{3} \mathrm{R}(3)$ methane manifold. Data acquired at ambient pressures, temperatures, and methane concentrations, with an optical path length of $5.79 \mathrm{~m}$.

Figure 4.6: Relationship between reference (L1) and absorption (L2) mean laser intensities using the basic system configuration, at ambient temperature and pressure, and at ambient methane volume mixing ratios. 108

Figure 4.7: The procedure to acquire a calibration data point ('run') 110

Figure 4.8: 'Real-time' WMS-based measurements (blue) overlaid on the requested test chamber methane volume mixing ratio measurement results (red) including 95\% uncertainties . 111

Figure 4.9: Sweep integration signal processing schematic. 113

Figure 4.10: Wavelength modulation spectroscopy signal processing schematic

Figure 5.1: Laser driving signal amplitude and frequency operation envelope as determined by hardware specification data

Figure 5.2: Surfaces indicating the normalized values of a) SNR, b) sensitivity, and c) the difference between the theory-based and measured absorption values using the SI technique. Data was obtained using the $2 v_{3} \mathrm{R}(3)$ methane manifold for a methane at a volume mixing ratio of $10 \mathrm{ppm}_{\mathrm{v}}$ in nitrogen, at standard atmospheric conditions. High contour values (red) indicate regions of desirable signal attributes. 
Figure 5.3: Surface indicating the equally-weighted sum of the normalized analysis parameters demonstrating the optimum SI sweep frequency and amplitude of $300 \mathrm{~Hz}$ and $1.0 \mathrm{~V}$. High contour values (highlighted in red) indicate regions of desirable signal attributes.

Figure 5.4: Surfaces indicating the normalized values of a) SNR, b) sensitivity, and c) the difference between the theory-based and measured $2 f$ values for the WMS technique (with no wavelength sweep). Data was obtained using the $2 v_{3} \mathrm{R}(3)$ methane manifold for a methane at a volume mixing ratio of $10 \mathrm{ppm}_{\mathrm{v}}$ in nitrogen, at standard atmospheric conditions. High contour values (highlighted in red) indicate regions of desirable signal attributes.

Figure 5.5: Surface indicating the equally-weighted sum of the normalized analysis parameters, demonstrating the optimum WMS signal frequency and amplitude at low frequencies and high amplitudes. High contour values (highlighted in red) indicate regions of desirable signal attributes.

Figure 5.6: Surface indicating $2 f$ value SNR for a one-second average over a range of WMS sweep signal frequencies and amplitudes. Data obtained with the test chamber purged with nitrogen at standard atmospheric conditions. High contour values (highlighted in red) indicate regions of desirable signal attributes.

Figure 5.7: Percent change in sensor head return intensity with time, relative to the pre-run calibration intensity. Data obtained with test chamber purged with nitrogen (at near standard ambient pressures and temperatures) while containing the $5.79 \mathrm{~m}$ sensor head, connected to the base station with a 5-m long, 9/125 $\mu \mathrm{m}$ fiber optic cable.

Figure 5.8: SI (a) and WMS (b) optical sensor head measurement deviation versus averaging time for various methods of estimating the index of the reference cell absorption or $2 f$ feature maximum; and their difference relative to using the (baseline) reference cell maximum value. Results calculated using over 100,000 sweep periods acquired over 12-hours, with the $5.79 \mathrm{~m}$ optical sensor head in the test chamber containing $25 \mathrm{ppm}_{\mathrm{v}}$ methane in air, at standard atmospheric temperature and pressure.

Figure 5.9: SI (top) and WMS (bottom) 1-second averaged optical sensor head measurement precision $(2 \sigma)$ and LDL for various combined methods of calibration and estimating the index of the reference cell $2 f$ or absorption feature maximum. Parameters calculated using over 600 measurements obtained at test chamber methane-in-air concentrations between 2.0 and $50.0 \mathrm{ppm}_{\mathrm{v}}$, acquired over a time-period spanning 8 hours

Figure 5.10: The difference in optical sensor head measurement deviation $(2 \sigma)$ for the leading $2 f$ and absorption features with and without digital RAM subtraction for the WMS (a) and SI (b) measurement techniques. This dataset is comprised of at least 800 seconds of absorption and $2 f$ data at each methane volume mixing ratio, each acquired over a 12hour period.

Figure 5.11: Inherent RAM suppression when using the leading and lagging portions of the returning intensity signal

Figure 5.12: Influence of RAM correction on measurement precision and LDL $(2 \sigma)$ for various calibration and absorption-free intensity estimation methods. Data acquired with the 
$5.79 \mathrm{~m}$ sensor head and test chamber methane concentrations between 2 and $100 \mathrm{ppm}_{\mathrm{v}}$ at ambient atmospheric temperature and pressure. 134

Figure 5.13: Measured absorption value deviation with and without intensity stabilization....... 136

Figure 5.14: Measured $2 f$ value deviation with and without intensity stabilization .................... 136

Figure 5.15: Plot of normalized a) SI absorption and b) WMS $2 f$ values with methods of mitigating polarization-dependant intensity fluctuations. Data obtained with test chamber methane concentrations between 2 and $25 \mathrm{ppm}_{\mathrm{v}}$ (at near standard ambient pressures and temperatures) while containing the $5.79 \mathrm{~m}$ sensor head, connected to the base station with

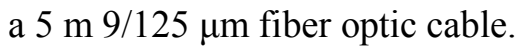
138

Figure 5.16: Influence of $9 / 125 \mu \mathrm{m}$ fiber-length on measured methane volume mixing ratio precision and mean drift for the SI method, using the concurrent method for estimating absorption-free intensity, at the $2 v_{3} \mathrm{R}(3)$ methane manifold at atmospheric temperature and pressure.

Figure 5.17: Influence of 9/125 $\mu \mathrm{m}$ fiber-length on measured methane volume mixing ratio precision and mean drift for the WMS method, using the concurrent and reference laser methods for estimating absorption-free intensity, at the $2 v_{3} \mathrm{R}(3)$ methane manifold at atmospheric temperature and pressure.

Figure 5.18: Measurement performance improvements for a SI-based system for various hardware and software system enhancements, for the $2 v_{3} \mathrm{R}(3)$ methane manifold at ambient temperature and pressure.

Figure 5.19: Measurement performance improvements for a WMS-based system for various hardware and software system enhancements, for the $2 v_{3} \mathrm{R}(3)$ methane manifold at ambient temperature and pressure.

Figure 5.20: Long-term system methane volume mixing ratio measurement precision and mean drift for the SI modulation scheme, using the concurrent method for estimating absorption-free intensity, for the $2 v_{3} \mathrm{R}(3)$ methane manifold at ambient temperature and pressure.

Figure 5.21: Long-term system methane volume mixing ratio measurement precision and mean drift for the WMS modulation scheme, using the concurrent and reference laser methods for estimating absorption-free intensity, for the $2 v_{3} \mathrm{R}(3)$ methane manifold at ambient temperature and pressure. 146

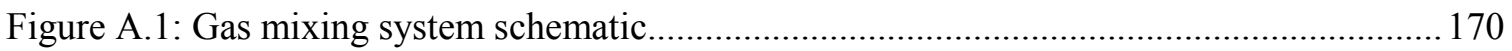

Figure A.2: Gas Mixing System Methane Concentration and Flow Rate Operating Range ....... 171

Figure A.3: Compressed air methane concentration observed over three days ......................... 173

Figure A.4: Residual between FTIR methane volume mixing ratio (VMR) measurements and requested methane VMR from the gas mixing system MFCs (averaged for 1 second) at a constant flow rate of 400 SLPM at ambient temperature and pressure. a) 0.5 SLPM MFC

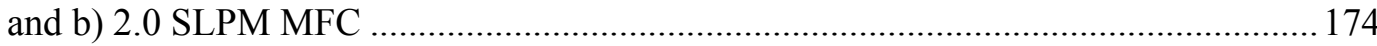

Figure A.5: Mixed gas methane volume mixing ratio uncertainty …...................................... 175

Figure A.6: $2 f$ Peak height response to step changes in requested concentration ........................ 176 
Figure A.7: Measured $2 f$ value settling time for requested concentration step-changes of 100 and $50 \mathrm{ppm}_{\mathrm{v}}$ at $200 \mathrm{SLPM}$

Figure B.1: SI look-up table interpolation error between 1 and $200 \mathrm{ppm}_{\mathrm{v}} \ldots \ldots \ldots \ldots \ldots \ldots \ldots \ldots \ldots \ldots \ldots . . . . . . . . . . . . . . . .179$

Figure B.2: WMS look-up table interpolation error between 1 and $200 \mathrm{ppm}_{\mathrm{v}}$

Figure B.3: Comparison of the methane volume mixing ratio predicted using the WMS and SI look-up tables (red and blue points respectively) with actual methane volume mixing ratios in the test chamber. The reference dashed black line is indicates perfect correspondence. Plotted data were obtained with test chamber pressures between $98 \mathrm{kPa}$ and $103 \mathrm{kPa}$, temperatures between $21^{\circ} \mathrm{C}$ and $25^{\circ} \mathrm{C}$, and with the $5.79 \mathrm{~m}$ optical sensor head coupled to the base station with a $6-\mathrm{m} 9 / 125 \mu \mathrm{m}$ fiber optic cable.

Figure B.4: Calibration residuals for SI method 1-second averaged methane volume mixing ratio values obtained using the theory-based calibration approach (which includes application of the empirical correction). Dashed and dotted lines indicate $95 \%$ prediction and confidence intervals of the final calibration. Plotted data were collected for ambient pressures and temperatures in the test chamber between $98 \mathrm{kPa}$ and $103 \mathrm{kPa}$ and $21^{\circ} \mathrm{C}$ and $25^{\circ} \mathrm{C}$. The thermal enclosure temperature was fixed at $20.1^{\circ} \mathrm{C}$ and data were acquired using the $5.79 \mathrm{~m}$ optical sensor head connected to the base station with a $5-\mathrm{m}$ long, $9 / 125 \mu \mathrm{m}$ fiber.

Figure B.5: Calibration residuals for WMS method 1-second averaged methane volume mixing ratio values obtained using the theory-based calibration approach (which includes application of the empirical correction). Dashed and dotted lines indicate $95 \%$ prediction and confidence intervals of the final calibration. Plotted data were collected for ambient pressures and temperatures in the test chamber between $98 \mathrm{kPa}$ and $103 \mathrm{kPa}$ and $21^{\circ} \mathrm{C}$ and $25^{\circ} \mathrm{C}$. The thermal enclosure temperature was fixed at $20.1^{\circ} \mathrm{C}$ and data were acquired using the $5.79 \mathrm{~m}$ optical sensor head connected to the base station with a $5-\mathrm{m}$ long, $9 / 125 \mu \mathrm{m}$ fiber.

Figure B.6: Calibration residuals for WMS method 1-second averaged methane volume mixing ratio values obtained using the experimental calibration approach. Dashed lines indicate 95\% prediction and confidence intervals of the final calibration function. Plotted data were collected for ambient pressures and temperatures in the test chamber between $98 \mathrm{kPa}$ and $103 \mathrm{kPa}$ and $21^{\circ} \mathrm{C}$ and $25^{\circ} \mathrm{C}$. The thermal enclosure temperature was fixed at $20.1^{\circ} \mathrm{C}$ and data were acquired using the $5.79 \mathrm{~m}$ optical sensor head connected to the base station with a 5-m long, 9/125 $\mu \mathrm{m}$ fiber. 185

Figure B.7: Calibration residuals for WMS method 1-second averaged methane volume mixing ratio values obtained using the experimental calibration approach. Dashed lines indicate $95 \%$ prediction and confidence intervals of the final calibration function. Plotted data were collected for ambient pressures and temperatures in the test chamber between $98 \mathrm{kPa}$ and $103 \mathrm{kPa}$ and $21^{\circ} \mathrm{C}$ and $25^{\circ} \mathrm{C}$. The thermal enclosure temperature was fixed at $20.1^{\circ} \mathrm{C}$ and data were acquired using the $5.79 \mathrm{~m}$ optical sensor head connected to the base station with a 5-m long, 9/125 $\mu \mathrm{m}$ fiber. 186

Figure B.8: Calibration residuals for WMS method 1-second averaged methane volume mixing ratio values obtained using the intensity-compensating experimental calibration approach. Dashed lines indicate $95 \%$ prediction and confidence intervals of the final calibration 
function. Plotted data were collected for ambient pressures and temperatures in the test chamber between $98 \mathrm{kPa}$ and $103 \mathrm{kPa}$ and $21^{\circ} \mathrm{C}$ and $25^{\circ} \mathrm{C}$. The thermal enclosure temperature was fixed at $20.1^{\circ} \mathrm{C}$ and data were acquired using the $5.79 \mathrm{~m}$ optical sensor head connected to the base station with a $5-\mathrm{m}$ long, $9 / 125 \mu \mathrm{m}$ fiber

Figure C.1: Measured variance of 1-second averaged values obtained at test-chamber methane-inair volume mixing ratios between 1 and $200 \mathrm{ppm}_{\mathrm{v}}$, with a polynomial best-fit. Data were acquired using the $2 v_{3} \mathrm{R}(3)$ methane manifold with test chamber pressures between $98 \mathrm{kPa}$ and $103 \mathrm{kPa}$, temperatures between $21^{\circ} \mathrm{C}$ and $25^{\circ} \mathrm{C}$, and with the $5.79 \mathrm{~m}$ optical sensor head coupled to the base station with a $6-\mathrm{m} 9 / 125 \mu \mathrm{m}$ fiber optic cable.

Figure C.2: Comparison of precision as determined by the $95 \%$ prediction interval of methane VMR measurements averaged for $1 \mathrm{~s}$ at test chamber methane volume mixing ratios between 2 and $50 \mathrm{ppm}_{\mathrm{v}}$, and long-run $2.5 \%$ and $97.5 \%$ percentiles at constant test chamber volume mixing ratios of $0,4.5,9.0,24.0$, and $48.5 \mathrm{ppm}_{\mathrm{v}}$ methane in air. Data were acquired using the $2 v_{3} \mathrm{R}(3)$ methane manifold with test chamber pressures between $98 \mathrm{kPa}$ and $103 \mathrm{kPa}$, temperatures between $21^{\circ} \mathrm{C}$ and $25^{\circ} \mathrm{C}$, and with the $5.79 \mathrm{~m}$ optical sensor head coupled to the base station with a $6-\mathrm{m} 9 / 125 \mu \mathrm{m}$ fiber optic cable.

Figure C.3: Schematic illustrating the Lower Detection Limit (LDL) and Limit of Detection (LOD) using the prediction and confidence intervals of a linear calibration function ... 193 


\section{Nomenclature}

Latin

First Use

\begin{tabular}{|c|c|c|c|c|}
\hline Symbol & Description & Units & Eq. & Pg. \\
\hline$A$ & Species-independent instrument-specific absorbance & $\mathrm{cm}^{-1}$ & 2.8 & 41 \\
\hline $\bar{A}$ & Laser current offset & $\mathrm{mA}$ & & 106 \\
\hline$a_{m}$ & Spectral modulation depth, a.k.a. modulation amplitude & $\mathrm{cm}^{-1}$ & 2.20 & 50 \\
\hline$a_{s}$ & Spectral sweep depth, a.k.a. sweep amplitude & $\mathrm{cm}^{-1}$ & 2.16 & 45 \\
\hline$b$ & Harmonic integer & - & & 45 \\
\hline$c$ & Speed of light in a vacuum, $c=299,792,458 \mathrm{~m} / \mathrm{s}$ & $\mathrm{m} / \mathrm{s}$ & 2.2 & 39 \\
\hline$d$ & Fiber-ring resonator (FRR) fiber-loop length & $\mathrm{m}$ & & 86 \\
\hline$E_{L}$ & Lower state energy - spectral constant & $\mathrm{cm}^{-1}$ & 2.4 & 40 \\
\hline$f$ & Frequency & $\mathrm{Hz}$ & & 102 \\
\hline$f_{m}$ & Modulation signal frequency & $\mathrm{Hz}$ & 2.19 & 102 \\
\hline$f_{s}$ & Sweep signal frequency & $\mathrm{Hz}$ & 2.15 & 102 \\
\hline$f_{u n}$ & Unwanted signal frequency & $\mathrm{Hz}$ & & 102 \\
\hline$g$ & Spectral line-shape function & $\mathrm{cm}$ & 2.5 & 41 \\
\hline$g_{G}$ & Gaussian line-shape function & $\mathrm{cm}$ & 2.10 & 42 \\
\hline$g_{L}$ & Lorentz line-shape function & $\mathrm{cm}$ & 2.11 & 42 \\
\hline$g_{V}$ & Voigt line-shape function & $\mathrm{cm}$ & 2.9 & 42 \\
\hline$G^{\prime}$ & $\begin{array}{l}\text { Sensitivity, the change in a measured absorption or } 2 \mathrm{f} \text { value } \\
\text { for a given change in methane volume mixing ratio }\end{array}$ & & & 116 \\
\hline$h$ & Planck's constant, $h=6.626 \times 10^{-34} \mathrm{~J} \cdot \mathrm{s}$ & $\mathrm{J} \cdot \mathrm{s}$ & 2.4 & 40 \\
\hline$H_{b}$ & $\begin{array}{l}\text { Fourier coefficients used to describe the transmittance for a } \\
\text { WMS signal }\end{array}$ & - & 2.26 & 53 \\
\hline$I$ & Measured light intensity with absorption & $\mathrm{V}$ & 2.1 & 28 \\
\hline$I_{0}$ & Measured light intensity without absorption & $\mathrm{V}$ & 2.1 & 28 \\
\hline$\overline{I_{0}}$ & $\begin{array}{l}\text { Mean light absorption-free intensity (evaluated over at least } \\
\text { one sweep period) }\end{array}$ & $\mathrm{V}$ & 2.15 & 45 \\
\hline$I_{0}{ }^{*}$ & $\begin{array}{l}\text { Estimate of the light intensity without absorption, } \\
\text { "absorption-free intensity" }\end{array}$ & $\mathrm{V}$ & 4.1 & 104 \\
\hline $\bar{I}_{0}^{*}$ & $\begin{array}{l}\text { Estimated mean absorption-free light intensity (evaluated } \\
\text { over at least one sweep period) }\end{array}$ & $\mathrm{V}$ & & 105 \\
\hline$I_{m}$ & $\begin{array}{l}\text { Return intensity in response to a high-frequency } \\
\text { modulation of the laser injection current signal with } \\
\text { absorption }\end{array}$ & $\mathrm{V}$ & & 56 \\
\hline$I_{0}$ WMS & $\begin{array}{l}\text { Absorption-free intensity in response to a high-frequency } \\
\text { modulation of the laser injection current signal }\end{array}$ & $\mathrm{V}$ & 2.21 & 50 \\
\hline$I_{\mathrm{WMS}}$ & $\begin{array}{l}\text { Return intensity in response to a combined sweep and high- } \\
\text { frequency modulation of the laser injection current signal } \\
\text { and absorption in the sensor head }\end{array}$ & $\mathrm{V}$ & & 52 \\
\hline $\bar{I}_{\mathrm{WMS}} \bar{I}_{S}$ & $\begin{array}{l}\text { Mean light intensity (evaluated over at least one } \\
\text { modulation period) }\end{array}$ & V & 4.3 & 107 \\
\hline${\overline{I_{0}}}_{\mathrm{WMS}}{ }^{*}$ & $\begin{array}{l}\text { Estimated mean light absorption-free intensity (evaluated } \\
\text { over at least one modulation period) }\end{array}$ & V & 4.3 & 107 \\
\hline
\end{tabular}


Latin

First Use

\begin{tabular}{|c|c|c|c|c|}
\hline Symbol & Description & Units & Eq. & Pg. \\
\hline$I_{s} I_{0_{S}}$ & $\begin{array}{l}\text { Return intensity in response to a low-frequency sweep of } \\
\text { the laser injection current signal with absorption }\end{array}$ & $\mathrm{V}$ & 2.17 & 47 \\
\hline $\bar{I}_{s}$ & $\begin{array}{l}\text { Mean return intensity in response to a low-frequency sweep } \\
\text { of the laser injection current signal with absorption } \\
\text { (evaluated over at least one sweep period) }\end{array}$ & $\mathrm{V}$ & 4.2 & 107 \\
\hline${\overline{I_{0}}}^{*}$ & $\begin{array}{l}\text { Estimated mean light absorption-free intensity (evaluated } \\
\text { over at least one sweep period) }\end{array}$ & $\mathrm{V}$ & 4.2 & 107 \\
\hline$I_{\text {RAM }}$ & Intensity due to residual amplitude modulation & V & & 56 \\
\hline$I_{0_{s}}$ & $\begin{array}{l}\text { Absorption-free intensity in response to a low-frequency } \\
\text { sweep of the laser injection current signal }\end{array}$ & V & 2.15 & 45 \\
\hline$i_{0_{m}}$ & $\begin{array}{l}\text { Light intensity amplitude of the of the modulation signal } \\
\text { first harmonic, normalized by } \bar{I}_{0}\end{array}$ & - & 2.19 & 50 \\
\hline$i_{0_{s}}$ & $\begin{array}{l}\text { Light intensity amplitude of the of the sweep signal first } \\
\text { harmonic, normalized by } \bar{I}_{0}\end{array}$ & - & 2.15 & 45 \\
\hline$i_{0}{ }^{*}$ & $\begin{array}{l}\text { Estimated light intensity amplitude of the of the modulation } \\
\text { signal first harmonic, normalized by } \overline{I_{0}}\end{array}$ & - & & 105 \\
\hline$i_{0_{s}}$ & $\begin{array}{l}\text { Absorption-free intensity in response to a low-frequency } \\
\text { "sweep" of the laser injection current signal }\end{array}$ & $\mathrm{V}$ & 2.15 & 45 \\
\hline$i_{2 m}$ & $\begin{array}{l}\text { Light intensity amplitude of the of the modulation signal } \\
\text { second harmonic, normalized by } \overline{I_{0}}\end{array}$ & - & 2.19 & 50 \\
\hline$i_{2_{s}}$ & $\begin{array}{l}\text { Light intensity amplitude of the of the sweep signal second } \\
\text { harmonic, normalized by } \bar{I}_{0}\end{array}$ & - & 2.15 & 45 \\
\hline$i_{2}{ }^{*}$ & $\begin{array}{l}\text { Estimated light intensity amplitude of the of the modulation } \\
\text { signal second harmonic, normalized by } \overline{I_{0}}\end{array}$ & - & & 105 \\
\hline $\bar{I}_{0}{ }_{\mathrm{L} 1}$ & $\begin{array}{l}\text { Mean estimated absorption-free light intensity for Laser } 1 \\
\text { (evaluated over at least one sweep period) }\end{array}$ & V & 4.4 & 108 \\
\hline $\bar{I}_{0_{\mathrm{L} 1}}$ & $\begin{array}{l}\text { Mean absorption-free light intensity for Laser } 1 \text { (evaluated } \\
\text { over at least one sweep period) }\end{array}$ & V & 4.4 & 109 \\
\hline $\bar{I}_{0}{ }_{\mathrm{L} 2}$ & $\begin{array}{l}\text { Mean estimated absorption-free light intensity for Laser } 2 \\
\text { (evaluated over at least one sweep period) }\end{array}$ & V & 4.4 & 108 \\
\hline $\bar{I}_{0 \mathrm{~L} 2}$ & $\begin{array}{l}\text { Mean absorption-free light intensity for Laser } 2 \text { (evaluated } \\
\text { over at least one sweep period) }\end{array}$ & $\mathrm{V}$ & 4.4 & 109 \\
\hline$\widehat{\bar{I}}_{0 \mathrm{~L} 2}$ & $\begin{array}{l}\text { Intercept of absorption-free estimate function relating } \\
\text { return intensity of Laser } 1 \text { to Laser } 2\end{array}$ & V & 4.4 & 108 \\
\hline$k$ & Boltzmann's constant, $k=1.380658 \times 10^{-19}\left(\mathrm{~kg} \cdot \mathrm{cm}^{2}\right) /\left(\mathrm{s}^{2} \cdot \mathrm{K}\right)$ & $\left(\mathrm{kg} \cdot \mathrm{cm}^{2}\right) /\left(\mathrm{s}^{2} \cdot \mathrm{K}\right)$ & 2.1 & 28 \\
\hline$L$ & $\begin{array}{l}\text { Optical path-length of laser beam through interrogation } \\
\text { region within optical sensor head }\end{array}$ & $\mathrm{m}$ & 2.1 & 28 \\
\hline$m$ & Mass of absorbing species & $\mathrm{g} / \mathrm{mole}$ & 2.12 & 42 \\
\hline$n$ & Index of refraction & - & & 86 \\
\hline$p$ & Pressure & $\mathrm{Pa}$ & 2.1 & 28 \\
\hline$p_{0}$ & HITRAN reference pressure, $p_{0}=101,325 \mathrm{~Pa}$ & $\mathrm{~Pa}$ & 2.3 & 40 \\
\hline$q$ & Species volume mixing ratio & $\mathrm{ppm}_{\mathrm{v}}$ & 2.1 & 28 \\
\hline$Q_{R V}$ & $\begin{array}{l}\text { Total internal partition sum function (TIPS) describing } \\
\text { statistical weightings of energy state levels for the molecule } \\
\text { of interest }\end{array}$ & - & 2.4 & 40 \\
\hline$R_{2 \mathrm{f}}$ & $\begin{array}{l}\text { Simulated } 2 f \text { signal from the wavelength modulation } \\
\text { spectroscopic measurement technique }\end{array}$ & - & 2.31 & 53 \\
\hline$S$ & $\begin{array}{l}\text { HITRAN parameter: Line strength describing the strength } \\
\text { of an absorption feature of a particular spectral line for an }\end{array}$ & $\begin{array}{l}\mathrm{cm}^{-1} \cdot \\
\left(\text { molecule } \cdot \mathrm{cm}^{-2}\right)^{-1}\end{array}$ & 2.4 & 40 \\
\hline
\end{tabular}




\begin{tabular}{|c|c|c|c|c|}
\hline Symbol & Description & Units & Eq. & Pg. \\
\hline$\overline{S_{0}}$ & $\begin{array}{l}\text { HITRAN constant: reference line strength of an absorption } \\
\text { feature at } p_{0} \text { and } T_{0}\end{array}$ & $\begin{array}{c}\mathrm{cm}^{-1} \cdot \\
\left(\text { molecule } \cdot \mathrm{cm}^{-2}\right)^{-1}\end{array}$ & 2.4 & 40 \\
\hline$s_{x}$ & $\begin{array}{l}\text { Standard-deviation of experimentally measured absorption } \\
\text { or } 2 f \text { values }\end{array}$ & - & & 116 \\
\hline$t_{s}$ & Sweep-period center time, corresponding to a sample index & $\mathrm{s}$ & 2.29 & 107 \\
\hline$T$ & Temperature of the species within the interrogation region & K & 2.1 & 28 \\
\hline$T_{0}$ & HITRAN reference temperature, $T_{0}=296 \mathrm{~K}$ & K & 2.4 & 40 \\
\hline$T_{c}$ & $\begin{array}{l}\text { Laser diode temperature which yields a mean output } \\
\text { wavelength at } v_{p}\end{array}$ & ${ }^{\circ} \mathrm{C}$ & & 105 \\
\hline$x_{2 f}$ & Value of $R_{2 f}\left(v_{p}\right)$ & - & & 56 \\
\hline$x_{\mathrm{abs}}$ & Value of $\alpha\left(v_{p}\right)$ & - & 2.18 & 47 \\
\hline $\bar{x}$ & $\begin{array}{l}\text { Mean of the measured absorption or } 2 f \text { values within a } \\
\text { dataset }\end{array}$ & - & & 116 \\
\hline$X_{2 f}$ & Even component of the simulated WMS signal & - & 2.27 & 53 \\
\hline$Y_{2 f}$ & Odd component of the simulated WMS signal & - & 2.28 & 54 \\
\hline
\end{tabular}

\begin{tabular}{|c|c|c|c|c|}
\hline \multirow{2}{*}{$\begin{array}{l}\text { Greek } \\
\text { Symbol }\end{array}$} & \multirow[b]{2}{*}{ Description } & \multirow[b]{2}{*}{ Units } & \multicolumn{2}{|c|}{ First Use } \\
\hline & & & Eq. & Pg. \\
\hline$\alpha$ & Absorption & - & 2.7 & 41 \\
\hline$\alpha_{L_{f}}^{0}$ & $\begin{array}{l}\text { HITRAN parameter: Air-broadened Lorentz half-width of a } \\
\text { particular spectral line for an absorbing species, at } T_{0}\end{array}$ & $\mathrm{~cm}^{-1} / \mathrm{atm}$ & 2.13 & 40 \\
\hline$\alpha_{L_{S}}^{0}$ & $\begin{array}{l}\text { HITRAN parameter: Self-broadened Lorentz half-width of } \\
\text { a particular spectral line for an absorbing species, at } T_{0}\end{array}$ & $\mathrm{~cm}^{-1} / \mathrm{atm}$ & 2.13 & 40 \\
\hline$\delta$ & $\begin{array}{l}\text { HITRAN parameter: Pressure-shift coefficient of a } \\
\text { particular spectral line for an absorbing species, at } T_{0}\end{array}$ & $\mathrm{~cm}^{-1} / \mathrm{atm}$ & 2.3 & 40 \\
\hline$\eta$ & Grouping of terms: $2 \pi f_{m} t$ & radians & 2.24 & 53 \\
\hline$\varphi$ & $\begin{array}{l}\text { Distance between reflective surfaces introducing optical } \\
\text { interference fringes }\end{array}$ & $\mathrm{m}$ & & 94 \\
\hline$\gamma$ & $\begin{array}{l}\text { HITRAN parameter: Lorentz half-width temperature } \\
\text { dependence coefficient }\end{array}$ & - & 2.13 & 40 \\
\hline$\lambda$ & Wavelength, $c / v$, often stated in $\mathrm{nm}$ & $\mathrm{m}$ & 2.2 & 39 \\
\hline$v$ & Wavenumber, $10^{-3} / \lambda$ & $\mathrm{cm}^{-1}$ & & 28 \\
\hline$v^{0}$ & $\begin{array}{l}\text { HITRAN parameter: Zero-pressure line-center of a } \\
\text { particular spectral line for an absorbing species, at } T_{0}\end{array}$ & $\mathrm{~cm}^{-1}$ & 2.3 & 40 \\
\hline $\bar{v}$ & $\begin{array}{l}\text { Average laser output wavenumber over a sweep or } \\
\text { modulation period }\end{array}$ & $\mathrm{cm}^{-1}$ & & 45 \\
\hline$v_{c}$ & Center wavenumber of a spectral absorption line & $\mathrm{cm}^{-1}$ & 2.4 & 28 \\
\hline$v_{p}$ & Wavenumber of the absorption manifold peak & $\mathrm{cm}^{-1}$ & 2.18 & 47 \\
\hline$v_{m}$ & $\begin{array}{l}\text { Laser output wavenumber resulting from modulation of } \\
\text { laser injection current }\end{array}$ & $\mathrm{cm}^{-1}$ & 2.20 & 50 \\
\hline$v_{s}$ & $\begin{array}{l}\text { Laser output wavenumber resulting from sweep of laser } \\
\text { injection current }\end{array}$ & $\mathrm{cm}^{-1}$ & 2.16 & 45 \\
\hline$v_{\mathrm{WMS}}$ & $\begin{array}{l}\text { Laser output wavenumber resulting from combined sweep } \\
\text { and modulation of laser injection current }\end{array}$ & $\mathrm{cm}^{-1}$ & 2.22 & 50 \\
\hline
\end{tabular}


Greek

First Use

\begin{tabular}{|c|c|c|c|c|}
\hline Symbol & Description & Units & Eq. & Pg. \\
\hline$\overline{\hat{\phi}}$ & $\begin{array}{l}\text { Relationship between the absorption (L1) and reference } \\
\text { (L2) laser return intensities. }\end{array}$ & - & 4.4 & 108 \\
\hline$\psi_{1 m}$ & $\begin{array}{l}\text { Phase-shift between the first-order modulation intensity and } \\
\text { wavelength signals }\end{array}$ & radians & 2.19 & 50 \\
\hline$\psi_{1_{s}}$ & $\begin{array}{l}\text { Phase-shift between the first-order sweep intensity and } \\
\text { wavelength signals }\end{array}$ & radians & 2.15 & 45 \\
\hline$\psi_{1}^{*}$ & $\begin{array}{l}\text { Estimated phase-shift between the first-order sweep or } \\
\text { modulation intensity and wavelength signals }\end{array}$ & radians & & 105 \\
\hline$\psi_{2 m}$ & $\begin{array}{l}\text { Phase-shift between the second-order modulation intensity } \\
\text { and wavelength signals }\end{array}$ & radians & 2.19 & 50 \\
\hline$\psi_{2_{s}}$ & $\begin{array}{l}\text { Phase-shift between the second-order sweep intensity and } \\
\text { wavelength signals }\end{array}$ & radians & 2.15 & 45 \\
\hline$\psi_{2}^{*}$ & $\begin{array}{l}\text { Estimated phase-shift between the first-order sweep or } \\
\text { modulation intensity and wavelength signals }\end{array}$ & radians & & 105 \\
\hline$\sigma_{v_{c}}$ & Absorption cross-section of an absorption line, at $v_{c}$ & $\mathrm{~cm}^{2} \cdot$ molecule & 2.1 & 28 \\
\hline$\tau$ & $\begin{array}{l}\text { Transmittance, the spectrally-dependant transmitted light } \\
\text { intensity }\end{array}$ & - & 2.1 & 28 \\
\hline
\end{tabular}

\begin{tabular}{|c|c|c|}
\hline Acronyms & Description & $\begin{array}{l}\text { Use } \\
\text { Pg. }\end{array}$ \\
\hline CEAS & Cavity-Enhanced Absorption Spectroscopy & 29 \\
\hline CRDS & Cavity Ring Down Spectroscopy & 29 \\
\hline DA & Direct Absorption & 30 \\
\hline DAQ & Data Acquisition System & 89 \\
\hline $\mathrm{FC} / \mathrm{APC}$ & Face-Centered/Angled Physical Contact & 75 \\
\hline FRR & Fiber-Ring Resonator & 77 \\
\hline FSR & Free Spectral Range & 86 \\
\hline GHG & Greenhous Gas & 21 \\
\hline GWP & Global Warming Potential & 21 \\
\hline HITRAN & High-Resolution Transmission Molecular Absorption Database & 37 \\
\hline HWHM & Half-Width Half-Maximum & 42 \\
\hline InGaAs & Indium gallium Arsenide & 79 \\
\hline IR & Infrared & 31 \\
\hline L1 & Laser 1 (reference laser) & 108 \\
\hline L2 & Laser 2 (absorption laser) & 108 \\
\hline LIF & Laser-Induced Fluorescence & 31 \\
\hline MEMS & Microelectromechanical Systems & 76 \\
\hline MFC & Mass-Flow Controller & 109 \\
\hline NI & National Instruments & 89 \\
\hline NICE-OHMS & Noise-Immune Cavity-Enhanced Optical-Heterodyne Molecular Spectroscopy & 30 \\
\hline PA & Photo-acoustic & 31 \\
\hline ppmv & Parts-per-million by volume & 28 \\
\hline RAM & Residual Amplitude Modulation & 56 \\
\hline SI & Sweep Integration & 30 \\
\hline SM & Single-Mode & 65 \\
\hline SNR & Signal-to-Noise Ratio & 71 \\
\hline
\end{tabular}


Use

\begin{tabular}{lll} 
Acronyms & Description & Pg. \\
\hline TDLAS & Tunable Diode Laser Absorption Spectroscopy & 25 \\
TEC & Thermoelectric Cooling & 43 \\
TIPS & Total Internal partition Sum Function & 40 \\
UNFCCC & United Nations Framework Convention on Climate Change & 21 \\
VOCs & Volatile Organic Compounds & 23 \\
WM & Wavelength Modulation & 49 \\
WMS & Wavelength Modulation Spectroscopy & 30
\end{tabular}

\begin{tabular}{lll} 
Chemical & & Use \\
Formulae & Description & Pg. \\
\hline $\mathrm{CO}_{2}$ & Carbon Dioxide & 21 \\
$\mathrm{H}_{2} \mathrm{O}$ & Water & 25 \\
$\mathrm{~N}_{2}$ & Nitrogen & 85
\end{tabular}




\section{Chapter 1 Introduction}

\subsection{Motivation - Fugitive Emission Detection in Gas-Processing plants}

This thesis research is motivated by the need for detection of fugitive methane emissions within oil and gas processing facilities. Fugitive emissions, defined as unintended or irregular leaks of gases and vapours generally from pressurized equipment and fittings, are an important source of air pollution that is difficult to monitor and control (US EPA, 2006). Within industrial facilities such as oil and gas processing plants, fugitive methane emissions can be a significant source of greenhouse gas (GHG) emissions (Kirchgessner et al., 1997; Chambers et al., 2008). In terms of climate-forcing potential, methane emission mitigation remains at the forefront of global efforts to reduce GHG emissions (IPCC, 2007; Ragnauth et al., 2013; GMI, 2010).

Greenhouse gas data reported by Annex I countries to the United Nations Framework Convention on Climate Change (UNFCCC) suggest that fugitive emissions from the upstream and downstream oil and natural gas sector accounted for $\sim 4.3 \%$ of global GHG emissions in 2012 (UNFCCC, 2014). In Canada, this same sector was estimated to contribute 177.3 Mt ( $\mathrm{CO}_{2}$ eq.) in 2013 (Environment Canada, 2014), which represents $24.4 \%$ of Canada's reported GHG emissions. Reported fugitive emissions (primarily emitted as methane and not including emissions from flaring and venting) accounted for more than $11.1 \%$ of the sector total, as illustrated in Figure 1.1. The estimated 19.7 Mt. of $\mathrm{CO}_{2}$ equivalent fugitive emissions represented $2.7 \%$ of Canada's total GHG emissions in 2013. It should be noted that these official estimates assume a 100-year time-horizon global warming potential (GWP) value for methane of 25 (Carras et al., 2006). If the more up-to-date GWP value of 36 (100-year time-horizon) or 87 (20year time-horizon) were used, as specified in the most recent International Panel on Climate Change (IPCC) assessment report (Myhre et al., 2013), the contributions of fugitive emission to overall global warming potential would be even more significant. In 
addition to these daunting statistics, fugitive emissions of flammable methane may pose safety concerns and the loss of saleable gas can result in lost revenues.

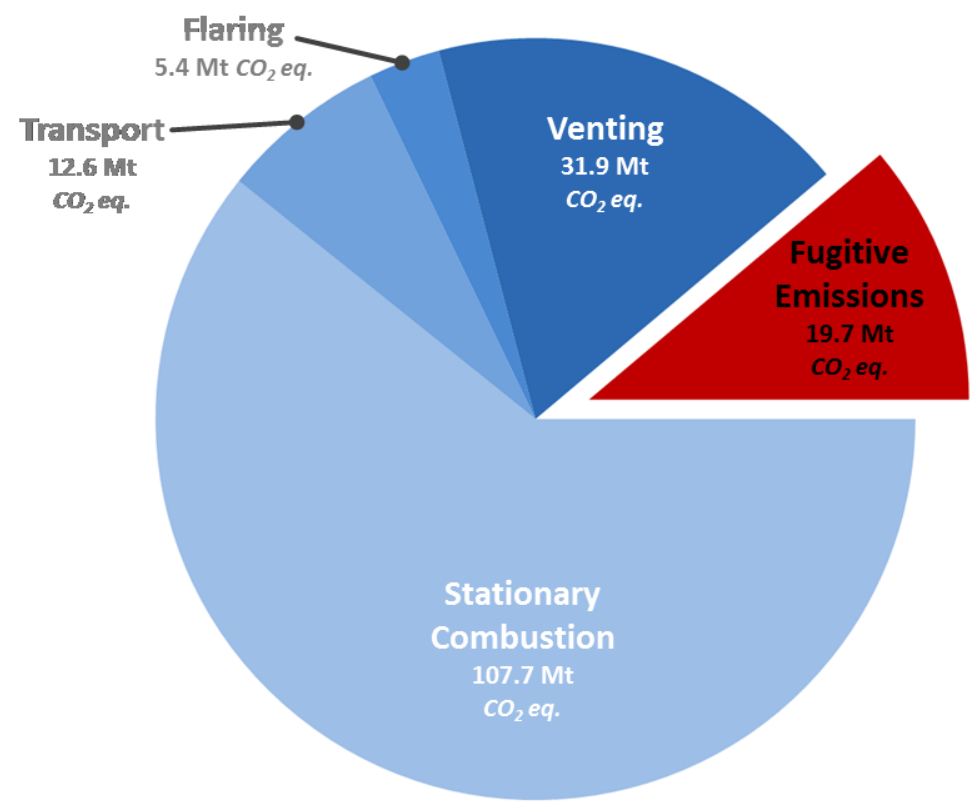

Figure 1.1: Total emissions by activity in Canada's Oil and Gas Sector. Data obtained from the 1990-2013 National Inventory Report (Environment Canada, 2014), which assumes a 100-year time-horizon GWP value for methane of 25.

Detection of fugitive methane is often difficult because the gaseous plumes emanating from individual sources are often intermittent and at low concentrations relative to ambient values of $\sim 1.7$ ppm $_{\mathrm{v}}$ (CAPP, 2007; Carras et al., 2006). Current methods of locating fugitive gas leaks most often involve qualitative manual methods, which can be tedious and costly, and can yield sporadic results. The most common qualitative detection techniques include:

- Surveys using infrared cameras, which enable visualization of infrared light absorption by the plume (US EPA, 2008);

- Manual application of soap-bubble solution to potential leak sources where creation of bubbles by escaping gas indicates the presence of a leak (EPA, 1995; Picard et al., 1998); 
- Use of "gas sniffers" generally based on metal oxide, catalytic, or electrochemical sensors which measure gas concentrations via resistance or current changes through the reacting surfaces (Gas Measurement Instruments Ltd., 2014) for which gas concentrations above a certain threshold (typically 10,000 ppm (US EPA, 2015)) are said to indicate the presence of a leak; and

- Use of open-path hand-held optical measurement devices generally based on infrared (IR) spectroscopy, which launch an IR laser beam thorough a plume and estimate gas concentration by observing reflected light from a nearby surface (Heath US, 2013).

Quantitative leak measurements can also be made by collecting leaks into bags and timing the fill rate or by using a Hi-Flow Sampler ${ }^{\mathrm{TM}}$, which attempts to suck the entire plume of a leak through a vacuum system and uses measured total flow rate and methane concentration to compute the leak rate (Bacharach Inc., 2002).

These methods are generally situation-specific and are not always directly suitable for fugitive emissions monitoring in an oil and gas processing facility. Often care has to be taken to limit interference from other gases, which may lead to false-readings or damaged sensors. The optimum solution for detecting fugitive emissions in a gas processing facility would be to combine the accuracy of laboratory-based techniques with the persistence, reliability, and safety associated with in situ measurement methods (Linnerud et al., 1998).

The two predominant in situ methane detection techniques are electrochemical and spectroscopic. Electrochemical or solid-state sensors have the advantage of being compact, reliable, and inexpensive. However, electrochemical sensors tend to have poor selectivity, are prone to contamination by other molecules, and may require considerable electrical power to heat the reactive surface - making them potentially dangerous to use in environments containing high concentrations of volatile organic compounds (VOCs) (Ameer and Adeloju, 2005; Chavali et al., 2008; Faglia et al., 1999; Lawrence, 2006; Opekar and Stulik, 2002; Ruiz-Garcia et al., 2009). Spectroscopic techniques are particularly sensitive and selective to many molecules that absorb energy within the 
visible and infrared ranges, but often require costly equipment and have not been widely embraced for large-scale, remote, in situ measurements (Uehara et al., 1997; Iseki, 2003; D'Amato, 2006).

A potentially viable method of monitoring fugitive emissions within an industrial facility would be to construct an array of sensors around the site, monitor ambient $\left(\sim 1.7 \mathrm{ppm}_{\mathrm{v}}\right)$ methane concentrations using spectroscopic techniques, and permit the estimation of fugitive leak location and emission rate by way of statistical and/or backtracking techniques (Brereton and Johnson, 2012; Joynes and Johnson, 2014). Recent simulations of this approach suggest that a measurement standard deviation of $2 \mathrm{ppm}_{\mathrm{v}}$ (equivalent to an absorbance of $8.1 \times 10^{-7} \mathrm{~cm}^{-1}$ for the $2 v_{3} \mathrm{R}(3)$ methane manifold at standard atmospheric pressure and temperatures) is sufficient to locate and potentially quantify fugitive methane sources using statistical methods (Brereton and Johnson, 2012), and possibly as high as $29 \mathrm{ppm}_{\mathrm{v}}$ using adjoint-based inverse dispersion modeling (Joynes, 2013).

\subsection{Objectives}

This thesis reports on the development and quantitative testing of a fiber-coupled, optically networked detection system designed for measuring in situ ambient methane concentrations within an industrial environment, such as an oil and gas processing plant. The system performance is assessed by:

- Quantifying volume mixing ratio (VMR) measurement accuracy and precision by measuring known-VMR methane-air and methane-nitrogen mixtures in a controlled laboratory setting (outlined in Sections 3.2, 4.6, 5.5), and

- Determining achievable lower detection limits by quantifying the minimum discernable spectroscopic signals (described in Sections 5.5).

The results and analysis also consider techniques for overcoming various interference, noise, and drift limitations common to spectroscopic systems, including: 
- Interference of other common infrared absorbing atmospheric absorbing gases (i.e. $\mathrm{H}_{2} \mathrm{O}, \mathrm{CO}_{2}$ ) (Section 2.3),

- System drift (Sections 3.1, 4.2, and 4.3),

- Residual amplitude modulation (Sections 2.2.2 and 5.3.2), and

- Optical interference (Section 3.2.1).

Lastly, the inferred ability of the developed system in performing in situ, long-term, continuous measurement of fugitive methane concentrations within an industrial environment is discussed (Section 5.5), and potential further improvements and approaches for possible extended capabilities are proposed (Chapter 6).

\subsection{Contribution}

This thesis builds upon various established tunable diode laser absorption spectroscopy (TDLAS) techniques to develop a system tailored for remotely measuring fluctuations in ambient methane. Major contributions include:

- Design of a unique optical fiber-coupled long optical path length sensor head for remote, multipoint quantification of atmospheric gases, which addresses commonly observed limitations such as stability, safety, and sensitivity. Unique design attributes include:

- a novel optical support structure design to promote long-term sensor stability, reliability, and fiber coupling efficiency (Section 3.1.2), and

○ bi-directional single-mode optical fiber coupling with a long optical pathlength sensor head to ensure high measurement sensitivity, remote measurements, intrinsically safe operation, and centralized signalprocessing (Section 3.1.2).

- A quantitative study on the effectiveness of various system drift management methods, commonly stated in publications as a limit to measurement precision and accuracy such as: 
- use of a second off-peak laser to provide an absorption-free intensity reference to aid in the quantification of losses within the system and fiber network (Section 4.3.1.2),

o polarization scrambling to mitigate the influence of fluctuations in polarization within the optical network and routing hardware (Section 3.1.3),

o thermal stabilization of system-critical optical components to reduce thermally-induced drift (Section 3.1.3), and

- automated system calibration routines to provide an estimate of the absorption-free laser characteristics (Section 4.3).

- Quantitative comparison of various methods of system calibration in terms of complexity and measurement performance (Section 5.2);

- Assessment of the influence of residual amplitude modulation on measurement performance and investigation of physical and digital suppression techniques (Section 2.2.2 and Section 5.3.2);

- Quantitative comparison of sweep integration and wavelength modulation spectroscopic measurement techniques (Section 5.5 ); and

- Design of a flexible system that can employ alternative spectroscopic measurements and fiber-coupled optical sensors without significant system alterations, for example the spectroscopic analysis of atmospheric gases in addition to methane (Section 3.1.1).

- A detailed description of a tunable diode laser-based spectroscopic system (operating in the near-infrared spectral region) and signal processing techniques which can be applied to other fiber-coupled optical sensing systems, including spectroscopic systems based on the rapidly-advancing field of quantum cascade lasers operating in the mid-infrared spectral region Section 2.1. 
Portions of this work have also been disseminated in journal (Schoonbaert et al., 2014a) and refereed conference proceedings (Schoonbaert et al., 2014b; Schoonbaert and Johnson, 2011).

\subsection{Outline}

Chapter 2 provides an introduction to various methane detection techniques, with a particular focus on the development and current state of tunable diode laser absorption spectroscopy. The design of the spectroscopic system, remote optical sensor, optical network, and test systems, are discussed in Chapter 3. Chapter 4 introduces the general signal processing methods used by this system, and includes specifics regarding methods of calibration and performance characterization. Chapter 5 presents performance and measurement results for specific system configurations and signal processing techniques, discusses the implications of these results, and makes suggestions on the optimum system for varying deployment configurations. Finally, Chapter 6 discusses the optimum system configuration for the quantification of remote methane within an industrial environment and makes suggestions regarding further research. 


\section{Chapter 2 Background and Absorption Spectroscopy Theory}

\subsection{Tunable Diode Laser Absorption Spectroscopy}

TDLAS is a method of accurately quantifying the spectrally-dependant radiative absorption of a gaseous mixture. This is achieved by tuning a laser diode light source over a spectral region containing absorption lines for a gas species of interest and quantifying the ratio between the laser intensity before and after absorption $\left(I_{0}\right.$ and $I[\mathrm{~V}]$ respectively, as measured by the data acquisition system), which is defined as the transmittance, $\tau$. The transmitted light intensity follows the Beer-Lambert-Bouguer Law which allows transmittance at a spectral wavenumber, $v\left[\mathrm{~cm}^{-1}\right]$, for an individual absorption line with center, $v_{c}$, to be calculated for a specified sample pressure, $p[\mathrm{~Pa}]$, temperature, $T[\mathrm{~K}]$, volume mixing ratio of the absorbing gas, $q[-]$, (although for clarity of presentation, $q$ is often quoted in parts-per-million by volume $\left[\mathrm{ppm}_{\mathrm{v}}\right]$ ), and optical path-length of the interfacing laser beam, $L[\mathrm{~m}]$ :

$$
\tau_{v_{c}}(v)=\frac{I}{I_{0}}=\exp \left(-\frac{q P L}{k T} \sigma_{v_{c}}(p, T, q, v)\right)
$$

where $k$ is Boltzmann's constant $\left[1.380658 \times 10^{-19}\left(\mathrm{~kg} \cdot \mathrm{cm}^{2}\right) /\left(\mathrm{s}^{2} \cdot \mathrm{K}\right)\right]$, and $\sigma_{v_{c}}$ is the absorption cross-section $\left[\mathrm{cm}^{2} /\right.$ molecule].

\subsubsection{Advancements in TDLAS}

In its infancy, the TDLAS technique was inefficient and cumbersome to implement, but yielded promising sensitivity and selectivity attributes lacking in other detection techniques. During the 1980s, TDLAS systems quickly emerged as a practical trace-gas sensing method for airborne atmospheric research (e.g. Hastie and Miller, 1985; Schiff et al., 1983; Webster, 1988) and were further refined through the development of field- 
deployable atmospheric research systems (D'Amato et al., 2002; Durry and Pouchet, 2002; Gardiner et al., 2010; Gurlit et al., 2005; Iseki, 2004; Nakaya et al., 2000; Richard et al., 2002; Simpson et al., 1995; Uehara et al., 1997; Wienhold et al., 1994).

In addition to applications in atmospheric research, TDLAS has become commonplace in aerospace, industrial, and internal combustion exhaust analysis (Baer et al., 1996; Liu et al., 2004b; Lyle, 2005; Rieker et al., 2007; Sanders et al., 2000). The development trend for TDLAS is moving away from laboratory-based systems toward in situ measurements and process monitoring. Additionally, the high demand for automated control and real-time chemical analysis has spurred the use of TDLAS in production and monitoring systems (Fernholz et al., 2002; Iseki, 2003; Johnstone et al., 2008; Linnerud et al., 1998; Simpson et al., 1995; So, 2008; Stewart et al., 1998a; Werle, 1998), and medical applications such as in vivo sensing, and breath analysis (Dahnke et al., 2001; Olesberg et al., 2006; Guan et al., 2009; Phillips, 1992; Zhang et al., 2013), pushing the sensitivity and uncertainty boundaries of the technology.

In general, when the volume mixing ratio of the absorbing gas is low, it must be resolved from a small change $\left(I_{0}-I\right)$ in a large signal $\left(I_{0}\right)$, which is easily obscured by signal noise. At atmospheric conditions there are two predominant methods for improving measurement sensitivity: increasing the optical path-length of the absorbing laser beam within an interrogation region, and reducing the signal noise.

TDLAS methods that aim to improve measurement sensitivity by increasing optical path length are often classified as cavity enhanced absorption spectroscopy (CEAS). Under this designation there are two methods of increasing optical path length within a confined volume or 'cell', either multipass or resonant. Multipass cells repeatedly redirect the laser beam through the interrogation region, and are usually mechanically simplistic but susceptible to mechanical misalignment and optical interference (Heriott et al., 1964; White, 1942; Silver and Stanton, 1988; Mohamed et al., 2013). Resonant cells increase the effective optical path length by matching the laser wavelength to a mode of an optical cavity formed by two or more highly reflective mirrors. Cavity ring-down spectroscopy (CRDS) (e.g. G Berden et al., 2000; Engeln et 
al., 1996; Romanini et al., 1997) and noise-immune cavity-enhanced optical-heterodyne molecular spectroscopy (NICE-OHMS) (Ye et al., 1998) are among two of the most promising resonant system configurations. Even though high sensitivities have been demonstrated with these systems (Paldus and Kachanov, 2005; He et al., 2014; Giel Berden et al., 2000; Dong et al., 2007; Orr and He, 2011; He et al., 2010), resonant cells are not suitable to this particular application due to their sensitivity to vibration, high resonant cavity cost, and the complexities associated with coupling light out of the cavity with optical fibers (Huang and Lehmann, 2010; He et al., 2014).

The second main method of increasing the measurement sensitivity is by reducing measurement noise. Shifting detection to higher frequencies, $f[\mathrm{~Hz}]$, has the potential to avoid a significant portion of noise within a spectroscopic system, referred to as $1 / f$ noise (Llopis et al., 2013; Brahimi et al., 2009; Werle et al., 1994; Werle et al., 1989). 1/f noise is introduced by active components within the electro-optical system (such as the laser diode and photodetector), where the noise occurs at frequencies that are inversely proportional to the frequency of the carrier signal. The influence of $1 / f$ noise is often supressed by modulating the temperature or driving current of a laser diode at high frequencies and extracting higher-order harmonics from the resultant detector signals (Reid and Labrie, 1981; Lindberg et al., 2001).

Detection techniques can be generally classified based on the driving signal of the laser. In the case where the laser driving signal is constant (or changed slowly using temperature tuning), the technique has been referred to as "direct absorption" (DA) (Hinkley, 1970). Alternatively, when the laser wavelength is swept over an absorption feature by a single low frequency driving signal (roughly $<5 \mathrm{kHz}$ ), the technique may be referred to as "sweep integration" (SI) (Cassidy and Reid, 1982a; Jennings, 1980). Lastly, when the laser is driven by a combined low- and high-frequency signal (where the high-frequency component of the driving signal is still significantly less than the optical frequency of the spectral feature half-width at half maximum) and the resultant secondharmonic (2f) or higher-order harmonics are observed, the technique is commonly referred to as wavelength modulation spectroscopy (WMS) (Silver, 1992; Bomse et al., 1992; Fernholz et al., 2002). 
TDLAS systems can be further classified in terms of the method by which the absorbed optical power is quantified. Most TDLAS systems implement some form of spectral semiconductor to directly measure the post-absorption intensity. However, in photo-acoustic (PA) spectroscopy or laser-induced fluorescence (LIF), the absorbed optical power can be measured indirectly, with pressure (acoustic) waves, or fluorescence of a target species. Though sensitive, these methods have limited applicability in industrial environments as they are limited by vibrational and acoustic noise, often require opto-electric type sensors, are affected by absorption from airborne particulates, and require detailed calibration (Giel Berden et al., 2000; Ye et al., 1998).

The ability to resolve spectral features, and hence achieve species selectivity, depends on the spectral coherence of the light source. The measurement of methane gas concentration using laser absorption spectroscopy was first developed by Moore (Moore, 1964) using a Helium-Neon laser observing radiative absorption in the infrared optical spectrum. Moore was limited by the available lasers at the time, expressing difficulties in finding radiation sources with narrow bandwidths that also overlapped with the absorption wavelengths of molecules of interest. Techniques and lasers used in laser spectroscopy were refined in the late $20^{\text {th }}$ century, with a variety of lasers operating within the mid-infrared (mid-IR) region $(2-25 \mu \mathrm{m})$, commonly constructed out of indium phosphate or lead-salts (Carlisle et al., 1989; Hinkley et al., 1976; Hinkley, 1970; Silver and Stanton, 1988). However, cryogenic cooling of sources and detectors hindered the practicality of such systems for field applications (Cassidy and Reid, 1982b; Duffin et al., 2007; Silver, 1992).

Methane has unique absorption lines in the near-IR $(0.7-2.0 \mu \mathrm{m})$ and mid-IR spectral regions that overlap with the output wavelengths for semiconducting lasers. In recent decades, semiconducting tunable diode lasers (TDLs) have become a popular choice for spectroscopic sensing due to their compact size, tuneability, and narrow spectral bandwidth at moderately-high continuous-wave output power. Semiconducting quantum cascade lasers (QCLs) have increased in commercial availability in recent years, and have been successfully used for spectroscopic sensing in the mid-IR spectral region (Smith et al., 2013; Kosterev et al., 2007; Pal et al., 2008; He et al., 2014; Wysocki et al., 
2007; Disselkamp et al., 2002; Sigrist et al., 2007). These lasers are operable at standard room temperatures, have a broad tuning range of up to $100 \mathrm{~cm}^{-1}$ mode-hop free, and have a high output power when compared to other semiconducting laser types. However, these lasers are currently not ideal for remote sensing via fiber optics due to the high fiber attenuation $(\sim 0.3 \mathrm{~dB} / \mathrm{m})$ and lack of fiber based optical components (such as couplers and circulators). Alternatively, fiber-coupled semiconducting lasers are popular for spectroscopic sensing in the near-IR (Ventrudo and Cassidy, 1990; Hinkley, 1970). Lasers and detectors and fibers within this region benefit from low cost and wide availability, spurred by their popularity in the telecom industry (Cassidy and Bonnell, 1988; Pavone and Inguscio, 1993). Standard telecom fibers operating in the near-IR (for example SMF-28e + ) have an extremely low attenuation of about $0.2 \mathrm{~dB} / \mathrm{km}(1000$ fold lower than currently available mid-IR fibers) lending themselves well to this application.

\subsubsection{Current state of TDLAS}

The versatility of the TDLAS technique and the growing availability of narrowbandwidth lasers, detectors, and fibers within the near- and mid-IR spectral regions have encouraged ongoing research and development. Table 2.1a and 2.1b summarize key details of recently published TDLAS systems employing various hardware topologies and targeting different gas species. The subset of published works listed in Table 2.1a included sufficient details to enable calculation of the precision of the measured absorbance (i.e. twice the standard deviation, $2 \sigma$ ), which, compared to the precision in the measured volume mixing ratio, removes dependence on instrument-specific optical pathlength and accounts for the differences in spectral line strengths. 
Table 2.1a: Details and Performance Characteristics of Current TDLAS Systems

\begin{tabular}{|c|c|c|c|c|c|c|c|c|}
\hline Reference & $\begin{array}{l}\text { Detection } \\
\text { Technique }^{\dagger}\end{array}$ & $\begin{array}{l}\text { Interrogation } \\
\text { Region Type }\end{array}$ & $\begin{array}{l}\text { Integrated } \\
\text { Detector? }\end{array}$ & $\begin{array}{l}\text { Optical } \\
\text { Path- } \\
\text { Length } \\
\text { [m] }\end{array}$ & $\begin{array}{l}\text { Conditions of } \\
\text { Validation }\end{array}$ & $\begin{array}{l}\text { Wavelength of } \\
\text { Estimated*** } \\
\text { Absorption Feature } \\
\text { Maximum* [nm] }\end{array}$ & $\begin{array}{c}\text { VMR } \\
\text { Standard } \\
\text { Deviation** } \\
(2 \sigma)\left[\mathrm{ppm}_{\mathrm{v}}\right]\end{array}$ & $\begin{array}{l}\text { Absorbance } \\
\text { Standard } \\
\text { Deviation*** } \\
(2 \sigma)\left[\mathrm{cm}^{-1}\right]\end{array}$ \\
\hline Berman et al., (2012) & CRDS & High-finesse, Flow-thru & Y & 4000 & $1.74 \mathrm{ppm}_{\mathrm{v}} \mathrm{CH}_{4}$ in Air & 1650.96 & 0.004 & $1.04 \times 10^{-9}$ \\
\hline Jin et al., (2015) & $2 f-$ WMS & Hollow Fiber & $\mathrm{N}$ & 10 & $10 \mathrm{ppm}_{\mathrm{v}} \mathrm{C}_{2} \mathrm{H}_{2}$ in $\mathrm{N}_{2}$ & 1530.37 & 0.004 & $2.30 \times 10^{-9}$ \\
\hline He et al. (2014) & CRDS & All-optical, Open-path & $\mathrm{N}$ & 0.36 & $9.0 \mathrm{ppm}_{\mathrm{v}} \mathrm{NH}_{3}$ in $\mathrm{N}_{2}$ & 1531.68 & 0.022 & $5.50 \times 10^{-9}$ \\
\hline Cao et al. (2013) & $2 f-\mathrm{PA}$ & All-optical, Micro-holes & $\mathrm{N}$ & 0.0015 & $1 \% \mathrm{C}_{2} \mathrm{H}_{2}$ in $\mathrm{N}_{2}$ & 1555.84 & 8.6 & $1.35 \times 10^{-8}$ \\
\hline Ren et al., (2014) & $2 f-W M S$ & Flow-thru & Y & 57.6 & $660 \mathrm{ppm}_{\mathrm{v}} \mathrm{CH}_{4}$ in $\mathrm{N}_{2}$ & 7802.68 & 0.0118 & $5.56 \times 10^{-8}$ \\
\hline Liu et al., (2015) & $2 f-W M S$ & Flow-thru & Y & 26.4 & $7.5 \mathrm{ppm}_{\mathrm{v}} \mathrm{CH}_{4}$ in $\mathrm{N}_{2}$ & 1653.73 & 0.158 & $6.03 \times 10^{-8}$ \\
\hline Precision Target & & & & & & & 2.0 & $8.10 \times 10^{-7}$ \\
\hline Nwaboh et al., (2013) & DA & Hollow Fiber & Y & 5.27 & $64 \mathrm{ppm}_{\mathrm{v}} \mathrm{CO}_{2}$ in $\mathrm{N}_{2}$ & 2005.09 & 118 & $1.44 \times 10^{-5}$ \\
\hline Bauer et al. (2014) & $2 f-\mathrm{PA}$ & Optical in/out, Flow-thru & $\mathrm{Y}$ & 0.01 & $100 \mathrm{ppm}_{\mathrm{v}} \mathrm{C}_{2} \mathrm{H}_{2}$ in $\mathrm{N}_{2}$ & 1532.83 & 15 & $1.60 \times 10^{-5}$ \\
\hline Tai et al. (1992) & $2 f-W M S$ & All-optical, Flow-thru & $\mathrm{N}$ & 0.1 & $350 \mathrm{ppm}_{\mathrm{v}} \mathrm{CH}_{4}$ in Air & 1665.96 & 104 & $4.11 \times 10^{-5}$ \\
\hline Stewart et al., (2010) & $2 f-$ WMS & All-optical, Open-path & $\mathrm{N}$ & 0.05 & $25 \mathrm{ppm}_{\mathrm{v}} \mathrm{CH}_{4}$ in Air & 1650.96 & 100 & $4.85 \times 10^{-5}$ \\
\hline Huang et al., (2014) & $2 f-$ WMS & Flow-thru & Y & 0.2 & $1000 \mathrm{ppm}_{\mathrm{v}} \mathrm{CH}_{4}$ in $\mathrm{N}_{2}$ & 1645.54 & 140 & $6.34 \times 10^{-5}$ \\
\hline Zhang et al., (2012) & DA & All-optical, Open-path & $\mathrm{N}$ & 0.1 & $1.0 \% \mathrm{CH}_{4}$ in Air & 1645.54 & 200 & $9.88 \times 10^{-5}$ \\
\hline Ye et al., (2015) & DA & Flow-thru & $\mathrm{Y}$ & 0.048 & 100 ppm $_{v} \mathrm{CH}_{4}$ in $\mathrm{N}_{2}$ & 3309.99 & 200 & $2.07 \times 10^{-4}$ \\
\hline Yu et al., (2001) & $2 f-W M S$ & All-optical & $\mathrm{N}$ & 0.025 & $1.0 \% \mathrm{C}_{2} \mathrm{H}_{2}$ in Air & 1530.37 & 330 & $3.86 \times 10^{-4}$ \\
\hline Ho et al., (2000) & $2 f-W M S$ & All-optical & $\mathrm{N}$ & 0.025 & $0.94 \% \mathrm{C}_{2} \mathrm{H}_{2}$ in Air & 1530.37 & 540 & $6.32 \times 10^{-4}$ \\
\hline Li, (2006) & $2 f-$ WMS & All-optical, Flow-thru & $\mathrm{N}$ & 0.1 & 790 ppm $_{v} \mathrm{CH}_{4}$ in Air & 1645.54 & 1500 & $7.44 \times 10^{-4}$ \\
\hline Chan et al., (1985) & DA & All-optical, Flow-thru & $\mathrm{N}$ & 0.5 & $1 \% \mathrm{CH}_{4}$ in Air & 1665.96 & 2632 & $1.04 \times 10^{-3}$ \\
\hline Smith et al., (2013) & $2 f-W M S$ & Flow-thru & Y & 0.008 & 384 ppm $_{\mathrm{v}} \mathrm{CO}_{2}$ in $\mathrm{N}_{2}$ & 4247.31 & 28 & $5.96 \times 10^{-3}$ \\
\hline
\end{tabular}


Table 2.1b: Details and Characteristics of Current TDLAS Systems (Continued from Table 2.1a)

\begin{tabular}{|c|c|c|c|c|c|c|c|c|}
\hline Reference & $\begin{array}{l}\text { Detection } \\
\text { Technique }^{\dagger}\end{array}$ & $\begin{array}{l}\text { Interrogation } \\
\text { Region Type }\end{array}$ & $\begin{array}{l}\text { Integrated } \\
\text { Detector? }\end{array}$ & $\begin{array}{l}\text { Optical } \\
\text { Path- } \\
\text { Length } \\
\text { [m] }\end{array}$ & $\begin{array}{l}\text { Conditions of } \\
\text { Validation }\end{array}$ & $\begin{array}{l}\text { Wavelength of } \\
\text { Estimated*** } \\
\text { Absorption Feature } \\
\text { Maximum* [nm] }\end{array}$ & $\begin{array}{c}\text { VMR } \\
\text { Standard } \\
\text { Deviation** } \\
(2 \sigma)\left[\text { ppm }_{\mathrm{v}}\right]\end{array}$ & $\begin{array}{l}\text { Absorbance } \\
\text { Standard } \\
\text { Deviation*** } \\
(2 \sigma)\left[\mathrm{cm}^{-1}\right]\end{array}$ \\
\hline Mappé et al., (2013) & DA & Flow-thru & $\mathrm{Y}$ & 76 & $0.307 \mathrm{ppm}_{\mathrm{v}} \mathrm{N}_{2} \mathrm{O}$ in $\mathrm{N}_{2}$ & & 0.00064 & \\
\hline Brumfield et al., (2015) & DA & Open-path & $Y$ & 127 & $\mathrm{~N}_{2} \mathrm{O}$ in $\mathrm{N}_{2}$ & 7190 to 7820 & & \\
\hline Kazemi et al., (2013) & Flourescence & Porous Fiber & $\mathrm{N}$ & & $0.2 \% \mathrm{O}_{2}$ in $\mathrm{N}_{2}$ & 3359.30 & & \\
\hline Khan and Kang, (2014) & Hetrodyne & Fiber & $\mathrm{N}$ & 0.0025 & $1 \mathrm{ppm}_{\mathrm{v}} \mathrm{VOC}$ in $\mathrm{N}_{2}$ & 1960 & & \\
\hline Krzempek et al., (2013) & $2 f-W M S$ & Flow-thru & $\mathrm{Y}$ & 57.6 & $0.1 \mathrm{ppm}_{\mathrm{v}} \mathrm{C}_{2} \mathrm{H}_{6}$ in $\mathrm{N}_{2}$ & 678 & & \\
\hline Leen et al., (2013) & $2 f-W M S$ & High-finesse, Flow-thru & $\mathrm{Y}$ & & $5 \mathrm{ppb}_{\mathrm{v}} \mathrm{NH}_{3}$ in dry Air & 1550 & 0.00018 & \\
\hline Manivannan et al., (2012) & DA & Fiber & $\mathrm{N}$ & 0.03 & $12.5 \mathrm{ppm} \mathrm{NH} \mathrm{NH}_{3}$ in $\mathrm{N}_{2}$ & 1634 to 1653 & & \\
\hline Martan et al., (2014) & DA & Hollow Fiber & $\mathrm{N}$ & 0.3 & $48.6 \mathrm{ppm}_{\mathrm{v}} \mathrm{C}_{7} \mathrm{H}_{8}$ in $\mathrm{N}_{2}$ & 1665.96 & & \\
\hline Rieker et al., (2014) & DA & Open-path, Telescope & $Y$ & 2000 & $1.8 \mathrm{ppm} \mathrm{CH}_{4}$ in Air & 2300 & 0.006 & \\
\hline Shemshad, (2015) & $2 f-W M S$ & Fixed gas cell & $Y$ & 0.165 & $0.1 \% \mathrm{CH}_{4}$ & 1620 & & \\
\hline Triki et al., (2015) & $2 f-\mathrm{PA}$ & Flow-thru & $\mathrm{Y}$ & & $1 \%$ ppmv $\mathrm{CH}_{4}$ in $\mathrm{N}_{2}$ & 3380.66 & 30 & \\
\hline
\end{tabular}

+ CRDS - Cavity Ring-Down Spectroscopy, DA - Direct Absorption, 2f-WMS Second-harmonic Wavelength Modulation Spectroscopy, PA - Photo-acoustic

* The calculated wavelength of most prominent manifold within $0.2 \mathrm{~nm}$ of the report center wavelength

** For the reported species

*** Calculated using the 2012 HITRAN database (Rothman et al., 2013) and Lorentz profiles at 101325 Pa and $296 \mathrm{~K}$ 
The blue shaded line in Table 2.1a indicates the present precision target for the proposed fugitive methane measurement application (See Section 1.1). Although some very recent TDLAS systems such as those listed above the blue shaded line have exceeded this performance target, they have done so using hardware and techniques which are not well suited for the target application which requires passive, multipoint sampling, in an industrial environment. For example, He et al. (2014) and Berman et al., (2012) demonstrated precise measurement of ammonia and methane respectively using CRDS based systems, but required high-quality mirrors and precise optical alignment to create the high-finesse cavity. The cost of many such CRDS systems installed around an industrial facility would be prohibitively expensive. While $\mathrm{He}$ et al., (2014) demonstrated the feasibility of uncoupling a high-finesse cavity from its base TDLAS system, this system used potentially costly, high-reflectivity mirrors requiring protection by purge gas, which could have complexity and cost implications in a multipoint system. In addition, mechanical vibrations can introduce challenges for the use of high-finesse cavities (Fox et al., 2002), which could further complicate the use of this approach in the target industrial application.

Like a majority of TDLAS systems for point concentration measurements appearing in the literature, the systems of Berman et al. (2012); Ren et al. (2014); and Liu et al. (2015), make use of flow-thru type interrogation cells that pump sample gas through a pressurized cell. Extracting sample gas from many remote locations within a gas plant would introduce many system complexities not well suited to this application, such as line-heating, sample conditioning (such as drying and filtering), continual pumping to improve response-time, and the associated systems for ensuring the safe handling of a potentially flammable gas mixture (Gasmet, 2015).

Cao et al. (2013) demonstrated an all-optical 2f-PA-based system, however the fragility of their diaphragm, potential sensitivity to external acoustic and vibrational noise (Ye et al., 1998), combined with the systems' reliance on diffusion through micro channels could limit its compatibility with continuous, time-resolved detection of ambient methane in an industrial environment. 
Jin et al., (2015) developed a system which is able to achieve outstanding measurement precision using a hollow-fiber optic as an interrogation cell. However this approach suffers from an inhibiting diffusion rate through micro-holes drilled in the fiber, limiting the applicability of this or similar hollow-fiber based methods (Nwaboh et al., 2013; Kazemi et al., 2013; Khan and Kang, 2014; Manivannan et al., 2012) for resolving the transient methane plumes expected in this application.

From the literature cited in Table 2.1a and 2.1b, there are potential combinations of hardware configurations and signal processing methods that could be used in a system tailored specifically for the application of passively quantifying ambient methane at many points throughout a large industrial facility. For instance, it is common for systems to pass a laser beam multiple times through the gas sample in order to increase absorption and reduce the subsequent measurement precision value (Liu et al., 2015; Ren et al., 2014; Mappé et al., 2013; Krzempek et al., 2013; Wei et al., 2015). Similarly it is common to locate the photodetector immediately after the interrogation region [i.e. (Berman et al., 2012; Ren et al., 2014; Liu et al., 2015; Nwaboh et al., 2013; Bauer et al., 2014; Huang et al., 2014; Ye et al., 2015; Smith et al., 2013; Mappé et al., 2013; Brumfield et al., 2015; Krzempek et al., 2013; Leen et al., 2013; Rieker et al., 2014; Shemshad, 2015; Triki et al., 2015)]. However, while having a detector as part of the interrogation cell improves tolerance to beam deviation and dispersion due to the larger target area on a photodiode versus a collimating optic, eliminating the photodetector from an interrogation region placed in situ reduces the need for electrical components and cabling, and more importantly for this application, removes the flammability risk by ensuring the remote sensor head is intrinsically safe. In principle, this might be implemented by centralizing of the expensive and sensitive base TDLAS system within an intrinsically safe environment, and multiplexing the laser signal to many sensor heads located hundreds of meters away using fiber optics (Dubaniewicz, 2006; Stewart et al., 1998a; Stewart et al., 2004). 


\subsubsection{Fiber-Optic Based TDLAS}

While spectroscopic measurement of atmospheric methane is an established technique, it has not often been extended to remote detection using fiber-coupled all-optical sensors. Although high sensitivity fiber-coupled opto-electric systems have been demonstrated [e.g. (Orr and He, 2011; He et al., 2010)], in an industrial environment where intrinsic safety is paramount, systems with remote sensors that do not require electricity are of particular interest. Table 2.2 summarizes published data for the subset of systems from Table 2.1 that utilized intrinsically safe, all-optical, fiber-coupled sensors for the measurement of gases at atmospheric pressures and temperatures and compares them to the precision and LDL targets set for this system. For each gas species, calculations were performed using spectral parameters from the 2012 high-resolution transmission molecular absorption database, HITRAN (Rothman et al., 2013), at the most prominent manifold within $0.2 \mathrm{~nm}$ of the reported center wavelength, and using a Lorentzian lineshape at $101.325 \mathrm{kPa}$ and $296 \mathrm{~K}$.

Since Chan et al. (1985) presented a fiber-coupled remote optical sensor constructed using microscope lenses for beam collimation, there has been steady improvement in the reported measurement standard deviation for fiber-coupled gas sensors. The most notable detection limits have been reported recently using systems implementing CRDS, second-harmonic wavelength modulation spectroscopy ( $2 f$-WMS), and second-harmonic photo-acoustic spectroscopy ( $2 f$-PA)

As mentioned in Section 2.1.2, He et al. (2014) demonstrated quantification of ammonia in nitrogen with a remote fiber-coupled CRDS based optical sensor, achieving a measurement deviation (in terms of absorbance) of approximately $1.1 \times 10^{-8} \mathrm{~cm}^{-1}$. Although sensitive and expandable to multiple all-optical sensors, the CRDS method requires high-quality mirrors and precise optical alignment to achieve the best measurement performance, necessitating compromises between sensitivity and sensor cost. 
Table 2.2: Performance Characteristics of Fiber-Based TDLAS Systems

\begin{tabular}{|c|c|c|c|c|c|}
\hline Reference & $\begin{array}{l}\text { Detection } \\
\text { Technique }^{\dagger}\end{array}$ & $\begin{array}{c}\text { Conditions of } \\
\text { Validation }^{\ddagger}\end{array}$ & $\begin{array}{c}\text { Wavelength of } \\
\text { Calculated*** } \\
\text { Absorption Feature } \\
\text { Maximum* [nm] }\end{array}$ & $\begin{array}{c}\text { VMR Standard } \\
\text { Deviation** } \\
(2 \sigma)\left[p p m_{v}\right]\end{array}$ & $\begin{array}{l}\text { Absorbance } \\
\text { Standard } \\
\text { Deviation } * * * \\
(2 \sigma)\left[\mathrm{cm}^{-1}\right] \\
\end{array}$ \\
\hline He et al. (2014) & CRDS & $9 \mathrm{ppm}_{\mathrm{v}} \mathrm{NH}_{3}$ in $\mathrm{N}_{2}$ & 1531.682 & 0.0216 & $1.1 \times 10^{-8}$ \\
\hline Cao et al. (2013) & $2 f-\mathrm{PA}$ & $1 \% \mathrm{C}_{2} \mathrm{H}_{2}$ in $\mathrm{N}_{2}$ & 1555.836 & 4.3 & $1.3 \times 10^{-8}$ \\
\hline Precision Target & & & & & $8.1 \times 10^{-7}$ \\
\hline Tai et al. (1992) & $2 f-W M S$ & $350 \mathrm{ppm}_{\mathrm{V}} \mathrm{CH}_{4}$ in Air & 1665.962 & $5^{b}$ & $4.0 \times 10^{-6}$ \\
\hline Bauer et al. (2014) & $2 f-\mathrm{PA}$ & 100 ppm $_{v} \mathrm{C}_{2} \mathrm{H}_{2}$ in $\mathrm{N}_{2}$ & 1532.831 & 2.5 & $5.3 \times 10^{-6}$ \\
\hline Stewart et al. (2010) & $2 f-W M S$ & $25 \mathrm{ppm}_{\mathrm{v}} \mathrm{CH}_{4}$ in Air & 1650.959 & $50^{c}$ & $4.8 \times 10^{-5}$ \\
\hline Zhang et al. (2012) & DA & $1.0 \% \mathrm{CH}_{4}$ in Air & 1645.543 & 100 & $9.9 \times 10^{-5}$ \\
\hline Yu et al. (2001) & $2 f-W M S$ & $1.0 \% \mathrm{C}_{2} \mathrm{H}_{2}$ in Air & 1530.371 & 165 & $3.9 \times 10^{-4}$ \\
\hline Ho et al. (2000) & $2 f-W M S$ & $0.94 \% \mathrm{C}_{2} \mathrm{H}_{2}$ in Air & 1530.371 & $270^{b}$ & $6.3 \times 10^{-4}$ \\
\hline Li (2006) & $2 f-W M S$ & $790 \mathrm{ppm}_{\mathrm{v}} \mathrm{CH}_{4}$ in Air & 1645.543 & $750^{b}$ & $7.4 \times 10^{-4}$ \\
\hline Chan et al. (1985) & DA & $1 \% \mathrm{CH}_{4}$ in Air & 1665.962 & $1315.8^{a}$ & $1.0 \times 10^{-3}$ \\
\hline \multicolumn{6}{|c|}{$\begin{array}{l}+C R D S-\text { Cavity Ring-Down Spectroscopy, DA - Direct Absorption, 2f-WMS Second-harmonic Wavelength Modulation } \\
\text { Spectroscopy, PA-Photo-acoustic }\end{array}$} \\
\hline
\end{tabular}

Bauer et al. (2014) demonstrated detection of acetylene in nitrogen using 2f-PA spectroscopy via a low-cost 3D-printed sensor (with integrated microphone), in which an absorbance precision of $5.3 \times 10^{-6} \mathrm{~cm}^{-1}$ was calculated based on measurements of $100 \mathrm{ppm}_{\mathrm{v}}$ acetylene in nitrogen. Similarly, Cao et al. (2013) demonstrated an all-optical $2 f$-PA-based system, achieving an absorbance precision of $1.3 \times 10^{-8} \mathrm{~cm}^{-1}$ acetylene in nitrogen. Even though these are promising advancements in remote optical $2 f$-PA indicating the viability of low-cost sensor solutions, the fragility of the diaphragm, sensitivity to external acoustic and vibrational noise (Ye et al., 1998), and its reliance on diffusion through micro channels could limit its compatibility with detection of ambient methane in an industrial environment.

Stewart et al. (2010) demonstrated the potential of $2 f$-WMS based remote multipoint open-path detection of methane in industrial environments, reporting on various iterations where performance was limited by optical fringes, and noise and system drift (Whitenett et al., 2003; Stewart et al., 1998a). Tai et al. (1992) presented a fiber-coupled 
continuous-wave $2 f$-WMS based system capable of achieving a minimum drift-limited detectable absorbance of $4.0 \times 10^{-6} \mathrm{~cm}^{-1}$, estimated from results obtained using an optically simplistic $10 \mathrm{~cm}$ fiber-coupled interrogation cell containing $350 \mathrm{ppm}_{\mathrm{v}}$ methane in nitrogen. The simplicity, selectivity, and theoretically achievable precision of the $2 f$ WMS technique shows promise for the remote monitoring of ambient methane, but requires specific attention to system design to achieve a target measurement precision and lower detection limit of less than $8.1 \times 10^{-7} \mathrm{~cm}^{-1}$ and $6.9 \times 10^{-7} \mathrm{~cm}^{-1}$ required for detecting and monitoring fugitive methane plumes (Brereton and Johnson, 2012).

Generally, systems which have achieved notable sensitivities applicable to remote fiber-based detection are those which increase absorption with CEAS combined with WMS (Weiguang et al., 2005; Cassidy and Reid, 1982b; Iseki, 2004). Common features used to improve measurement sensitivity in these systems include long averaging times, residual amplitude modulation suppression, optical interference (and background signal) subtraction, and correction for absorption-free intensity fluctuations which cause measurement drift (Smith et al., 2013; Huang et al., 2014; Berman et al., 2012; Martan et al., 2014; Rieker et al., 2014; Shemshad, 2015; Leen et al., 2013; Brumfield et al., 2015). It has been observed that published fiber-based systems do not implement many of the techniques for measurement improvement that are often utilized in single-point systems, as presented in Section 2.1.1. For this reason it was concluded that a TDLAS system which implements fiber-optics for remote detection of methane must be purpose-built, combining techniques normally observed in laboratory-demonstrated systems, and be characterized at low methane concentrations to properly demonstrate the ability to perform fast and sensitive measurements of fugitive emissions.

\subsection{Absorption Spectroscopy}

The following overview of spectroscopic absorption theory has been adapted from the formulations and nomenclature found in (Rothman et al., 1998; Gordley et al., 1994; Spectralcalc, 1994). Spectroscopic detection relies on the ability of gases to absorb light at specific optical frequencies, $f[\mathrm{~Hz}]$, or wavelengths, $\lambda[\mathrm{m}]$, related by the speed of light, $c[\mathrm{~m} / \mathrm{s}]$, according to: 


$$
\lambda=\frac{c}{v}
$$

To be consistent with the units used in the HITRAN2008 database (Rothman et al., 2009), wavenumber units are used, $v\left[\mathrm{~cm}^{-1}\right]$, where $v=10^{-3} / \lambda$. The theoretical absorption calculations make use of the spectral parameters listed in Table 2.3, which are provided in the HITRAN 2008 database (Rothman et al., 2005; Rothman et al., 2009) and tabulated at a reference pressure and temperature $\left(p_{0}=101.325 \mathrm{kPa}, T_{0}=296 \mathrm{~K}\right)$.

Table 2.3: HITRAN Parameters

\begin{tabular}{l|c|l}
\hline \multicolumn{1}{c|}{ Parameter } & Symbol & \multicolumn{1}{c}{ Units } \\
\hline Zero-pressure line-center & $v^{0}$ & $\mathrm{~cm}^{-1}$ \\
Air broadened Lorentz half-width & $\alpha_{L_{f}}^{0}$ & $\mathrm{~cm}^{-1} /$ atm at $T_{0}$ \\
Self-broadened Lorentz half-width & $\alpha_{L_{S}}^{0}$ & $\mathrm{~cm}^{-1} /$ atm at $T_{0}$ \\
Lower state energy & $E_{L}$ & $\mathrm{~cm}^{-1}$ \\
Line Strength & $S_{0}$ & $\mathrm{~cm}^{-1} /\left(\right.$ molecule $\left.\cdot \mathrm{cm}^{-2}\right)$ at $T_{0}$ \\
Pressure shift coefficient & $\delta$ & $\mathrm{cm}^{-1} /$ atm at $T_{0}$ \\
Half-width temperature dependence & $\gamma$ & - \\
\hline
\end{tabular}

The center wavenumber of each absorption line, $v_{c}$, can be estimated using the pressure shift coefficient, $\delta$, and is affected by the difference between the absorbing sample pressure, $p[\mathrm{~Pa}]$, and the reference pressure, $p_{0}$, by:

$$
v_{c}=v^{0}+\delta \frac{p}{p_{0}}
$$

In addition, the temperature affects the line strength, $S\left(T, v_{c}\right)$, according to:

$$
S\left(T, v_{c}\right)=S_{0} \frac{Q_{R V}\left(T_{0}\right)}{Q_{R V}(T)} \frac{\exp \left(-h c E_{L} / k T\right)}{\exp \left(-h c E_{L} / k T_{0}\right)} \frac{\left(1-\exp \left(-h c v_{c} / k T\right)\right)}{\left(1-\exp \left(-h c v_{c} / k T_{0}\right)\right)}
$$

where $h$ is Planck's constant $\left(6.626 \times 10^{-34} \mathrm{~m}^{2} \cdot \mathrm{kg} / \mathrm{s}\right)$ and $Q_{R V}$ is the "total internal partition sum function" (TIPS) which describes the statistical weightings of the state energy levels for the particular molecule of interest (Fischer, 2003; Rothman et al., 2005). The value of $Q_{R V}$ at a specified temperature, $T[\mathrm{~K}]$, is interpolated using a four point Lagrange polynomial from tabulated values available in HITRAN. 
The absorption cross-section, $\sigma_{v_{c}}\left[\mathrm{~cm}^{2} /\right.$ molecule], for a particular absorption line centered at $v_{c}$, is the product of the line strength, $S\left(T, v_{c}\right)$ [cm/molecule], and line-shape, $g\left(p, T, q, v-v_{c}\right)[\mathrm{cm}]$,

$$
\sigma_{v_{c}}(p, T, q, v)=S\left(T, v_{c}\right) g\left(p, T, q, v-v_{c}\right)
$$

To describe the transmittance at a given wavenumber, $\tau(v)$, the absorption crosssection of all contributing absorption lines (Gordley et al., 1994; Tyner, 2015; D'Amato, 2006) must be taken in to account (as further detailed in Section 2.3),

$$
\tau(v)=\exp \left(-\sum_{j} \sum_{i} \frac{q_{j} p L}{k T} \sigma_{v_{c_{i}}}\left(p, T, q_{j}, v\right)\right)
$$

where $\sigma_{v_{c_{i}} j}$ is the cross-section of the (ith $)$ absorption line center for the $\left(j^{\text {th }}\right)$ gas species of interest, $k$ is Boltzmann's constant $\left[1.380658 \times 10^{-19}\left(\mathrm{~kg} \cdot \mathrm{cm}^{2}\right) /\left(\mathrm{s}^{2} \cdot \mathrm{K}\right)\right], q$ is the volume mixing ratio (VMR, also sometimes called "concentration"), and $L[\mathrm{~m}]$ is the optical path-length. The absorption, $\alpha$, at $v$ is given by

$$
\alpha(v)=1-\tau(v)
$$

The performance of spectroscopic systems employing differing hardware and operating at different wavelengths can be compared fairly using the absorbance, $A\left[\mathrm{~cm}^{-1}\right]$, defined by

$$
A(v)=\sum_{j} \sum_{i} \frac{q_{j} p}{k T} \sigma_{v_{c_{i}}}\left(p, T, q_{j}, v\right)
$$

instead of a ppm based precision value, as $A$ removes dependence on instrument-specific optical path-length and accounts for the differences in spectral line strengths.

The observed absorption at a given wavelength is the combination of absorption from many absorption lines, and is a result of molecular motion and collisions perturbing the discrete energy at specific optical frequencies into distributions of energy over a 
range of frequencies. For methane at atmospheric conditions, broadened absorption lines can be approximated with a Voigt line shape (Reid and Labrie, 1981; Gordley et al., 1994), $g_{V}\left(v-v_{c}\right)$, defined by,

$$
\begin{aligned}
& g_{V}\left(v-v_{c}\right) \\
& =\frac{1}{H W H M_{G}} \sqrt{\frac{\ln (2)}{\pi}} \frac{\sqrt{\ln (2)}}{\pi} \frac{H W H M_{L}}{H W H M_{G}} \int_{-\infty}^{\infty} \frac{\exp \left(-t^{2}\right)}{\left(\sqrt{\ln (2)} \frac{v-v_{c}}{\alpha_{G}}-t\right)^{2}+\left(\sqrt{\ln (2)} \frac{H W H M_{L}}{H W H M_{G}}\right)^{2}} \mathrm{~d} t
\end{aligned}
$$

The Voight line shape is a convolution of the Gaussian line shape, $g_{G}\left(v-v_{c}\right)$, and the Lorentz line shape, $g_{L}\left(v-v_{c}\right)$, which describe the Doppler temperature and pressure-induced collision broadening (D'Amato, 2006) respectively as

$$
\begin{gathered}
g_{G}\left(v-v_{c}\right)=\frac{1}{H W H M_{G}} \sqrt{\frac{\ln (2)}{\pi}} \exp \left(-\ln (2)\left(\frac{v-v_{c}}{H W H M_{G}}\right)^{2}\right) \\
g_{L}\left(v-v_{c}\right)=\frac{1}{\pi} \frac{H W H M_{L}}{\left(v-v_{c}\right)^{2}+H W H M_{L}{ }^{2}}
\end{gathered}
$$

The half-width at half-maximum (HWHM, $\left[\mathrm{cm}^{-1}\right]$ ) for the Gaussian and Lorentz (denoted by subscripts $G$ and $L$ respectively) line-shapes follow (Gordley et al., 1994):

$$
\begin{gathered}
H W H M_{G}=v_{c} \sqrt{\frac{2 k T}{m c^{2}}} \\
H W H M_{L}=\left((1-q) \alpha_{L_{f}}^{0}+q \alpha_{L_{s}}^{0}\right) \frac{p}{p_{0}}\left(\frac{T_{0}}{T}\right)^{\gamma}
\end{gathered}
$$

where $m$ is the mass of the gas species in atomic mass units, $\gamma[-]$ is the half-width temperature dependence correction factor, and $\alpha_{L_{f}}^{0}$ and $\alpha_{L_{s}}^{0}\left[\mathrm{~cm}^{-1} / \mathrm{atm}\right]$ are the air- and self-broadened Lorentz half-width coefficients (Table 2.3). To better approximate experimentally-observed spectral absorption away from $v_{c}$, the theoretical Voigt lineshape, the profile can be scaled by (Gordley et al., 1994): 


$$
\frac{v}{v_{c}} \frac{\tanh (h c v / 2 k T)}{\tanh \left(h c v_{c} / 2 k T\right)} V\left(v-v_{c}\right)
$$

\subsubsection{Laser Driving Signals and Associated Spectroscopic Techniques}

A laser diode can be tuned across a spectral region of interest using direct thermal or drive current control of the laser diode. Thermal tuning is generally implemented using thermoelectric cooling (TEC) with a Peltier element, and is usually limited to frequencies of less than $1 \mathrm{~Hz}$ but can provide a tuning range of $20 \mathrm{~cm}^{-1}$ or greater. By contrast, current tuning can accommodate much higher frequencies (into the $\mathrm{GHz}$ range), but with a lower spectral tuning range $\left(<1 \mathrm{~cm}^{-1}\right)$. As mentioned previously, spectroscopic techniques can be classified in terms of the way in which the laser is spectrally tuned, and is discussed further here.

\subsubsection{Direct Absorption}

Direct absorption (DA) spectroscopy refers to the most simplistic method of spectroscopic analysis, whereby absorption is measured at a fixed wavelength; usually at the spectral center of a particular absorption manifold. DA based TDL systems were originally used to demonstrate the spectroscopic measurement technique (Hinkley et al., 1976; Inaba et al., 1979). Basic DA systems are often designed as proof-of-concept or low-cost spectroscopic solutions for 'alarm' type monitoring rather than high-sensitivity quantitative measurements (K Chan et al., 1984). Advanced DA systems take advantage of the significantly faster data acquisition rates, limited by acquisition hardware rather than the laser modulation rate, permitting low-latency spectroscopic measurements (Baer et al., 1996; Sanders et al., 2000).

Some DA systems combine refracted broadband sources and linear photodetector arrays for absorption measurements over a wide wavelength range (Richter et al., 2000; Stutz and Platt, 1996). Even though this method can be accurate for broad absorption features, there are significant resolution challenges in obtaining quantitative absorption spectra over the relatively narrow methane absorption manifolds in the near-IR region. Narrow-bandwidth lasers are difficult to stabilize at a specific wavelength using strictly 
open-loop control due to unavoidable drift of the diode temperature and injection current, and are often stabilized using a combination of thermal and current control ( $\mathrm{Hu}$ et al., 1993; Richter et al., 2000). Closed-loop control is preferable to open-loop control, but with an unmodulated narrow-bandwidth laser source, spectral analysis requires sensitive high-resolution equipment.

\subsubsection{Sweep Integration}

Sweep integration (SI) spectroscopy refers to systems that modulate or 'sweep' the laser output wavelength over an absorption feature of interest. Depending on the application, this may offer an accuracy improvement over the fixed-wavelength narrow-bandwidth DA method, since absorption can be interpolated over a range of wavelengths rather than being limited to measurement at a single point.

Systems classified as using sweep integration generally sweep the input TDL current at frequencies less than $2.5 \mathrm{kHz}$ (Cassidy and Reid, 1982b; Hayward et al., 1989; Silveira and Grasdepot, 1995). The sweep signal can be nearly any waveform; from standard waveforms such as sinusoid and saw-tooth (Nagali et al., 1996; Saarela et al., 2009), to more custom waveforms such as a highly-skewed ramp (Baer et al., 1996) and arbitrary waveforms (So et al., 2006; Saarela et al., 2009). The waveform type and frequency are generally optimized for the specific application and depend heavily on the combination of laser, controller, and signal generators used.

Laser diode driving signals other than pure sinusoids tend to have higher signal noise-floors due to the necessary inclusion of many frequencies required to generate complex waveforms (Hayward et al., 1989; Saarela et al., 2009; Rieker et al., 2009). This leads to increased difficulties when attempting to filter the resultant detector signals as wells as complexities when attempting to characterize system performance based on modulation inputs. For these reasons, sinusoidal signals are used in the present work to vary the laser diode current, as illustrated in the system schematic in Figure 2.1. 


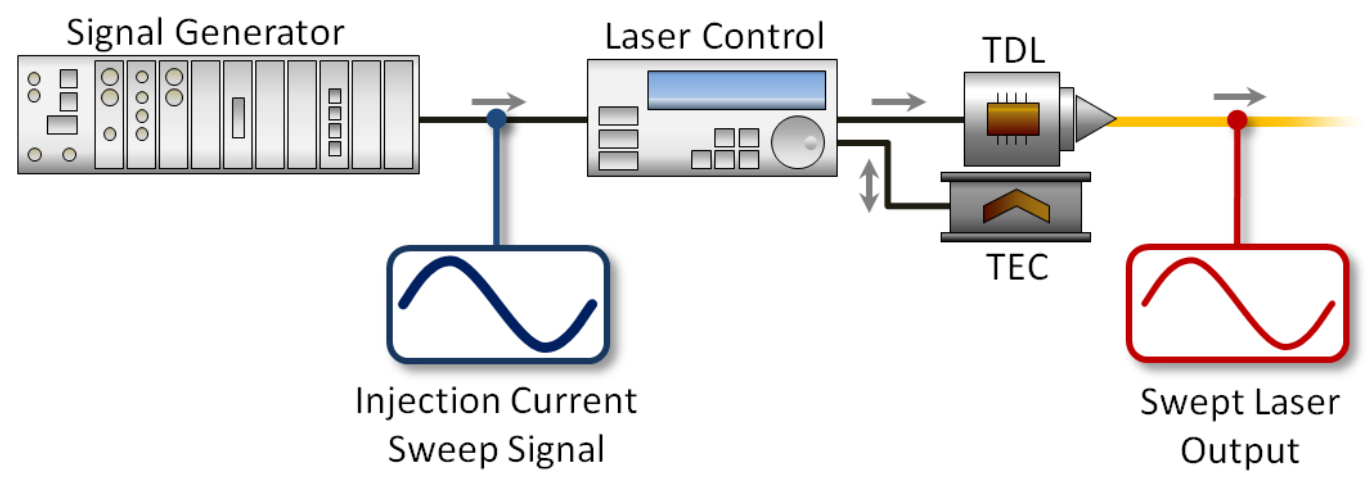

Figure 2.1: System schematic illustrating a low-frequency sweep injection current resulting in a low-frequency intensity and wavelength output

The TDL response to the injection current sweep signal is a time-varying intensity signal, $I_{0_{S}}(t)[\mathrm{V}]$, in addition to the desired wavenumber (or optical frequency) signal, $v_{s}(t)\left[\mathrm{cm}^{-1}\right]$. The laser output intensity and wavelength signals can be described by harmonics of the sinusoidal injection current input signal frequency $(b f$, where $b=1,2,3 \ldots$ and $1 f$ is the fundamental sweep frequency, $f_{s}$ ), the amplitudes of which decrease with increasing order. In practice, the laser output intensity signal can be well described by the first two harmonic terms (i.e. intensity at the sweep frequency $1 f_{s}$ and twice the sweep frequency, $2 f_{s}$ ), while the wavenumber signal can be described by the first harmonic (i.e. wavenumber at $1 f_{s}$ ). Higher harmonics for both signals tend to fall below the signal noise. The instantaneous optical frequency and laser intensity can be modeled by:

$$
\begin{gathered}
I_{0_{s}}(t)=\bar{I}_{0}\left(1+i_{0_{s}} \cos \left(2 \pi f_{s} t+\psi_{1_{s}}\right)+i_{2_{s}} \cos \left(4 \pi f_{s} t+\psi_{2_{s}}\right)\right) \\
v_{s}(t)=\bar{v}+a_{s} \cos \left(2 \pi f_{s} t\right)
\end{gathered}
$$

where $f_{s}$ is the sweep frequency, $\bar{v}$ is the average laser wavenumber, $a_{s}$ is the spectral depth of the sweep $\left[\mathrm{cm}^{-1}\right], \bar{I}_{0}$ is the average detected laser intensity [V], and $i_{0_{S}}, i_{2_{S}}$ are the first-and second-order sweep intensity amplitudes, at $f_{s}$ and $2 f_{s}$ respectively. In addition, $\psi_{1_{s}}$ and $\psi_{2_{s}}$ account for the phase shifts between the first- and second-order sweep intensity and wavelength signals respectively. Figure 2.2 demonstrates a typical absorption-free experimental SI laser return signal resulting from a low-frequency $10 \mathrm{~mA}$ 
$(0.5 \mathrm{~V})$ injection current sweep combined with a constant current offset of $90 \mathrm{~mA}$, at $\bar{v}=$ $1653.73 \mathrm{~nm}$ (corresponding to the $2 v_{3} \mathrm{R}(3)$ methane absorption manifold peak).

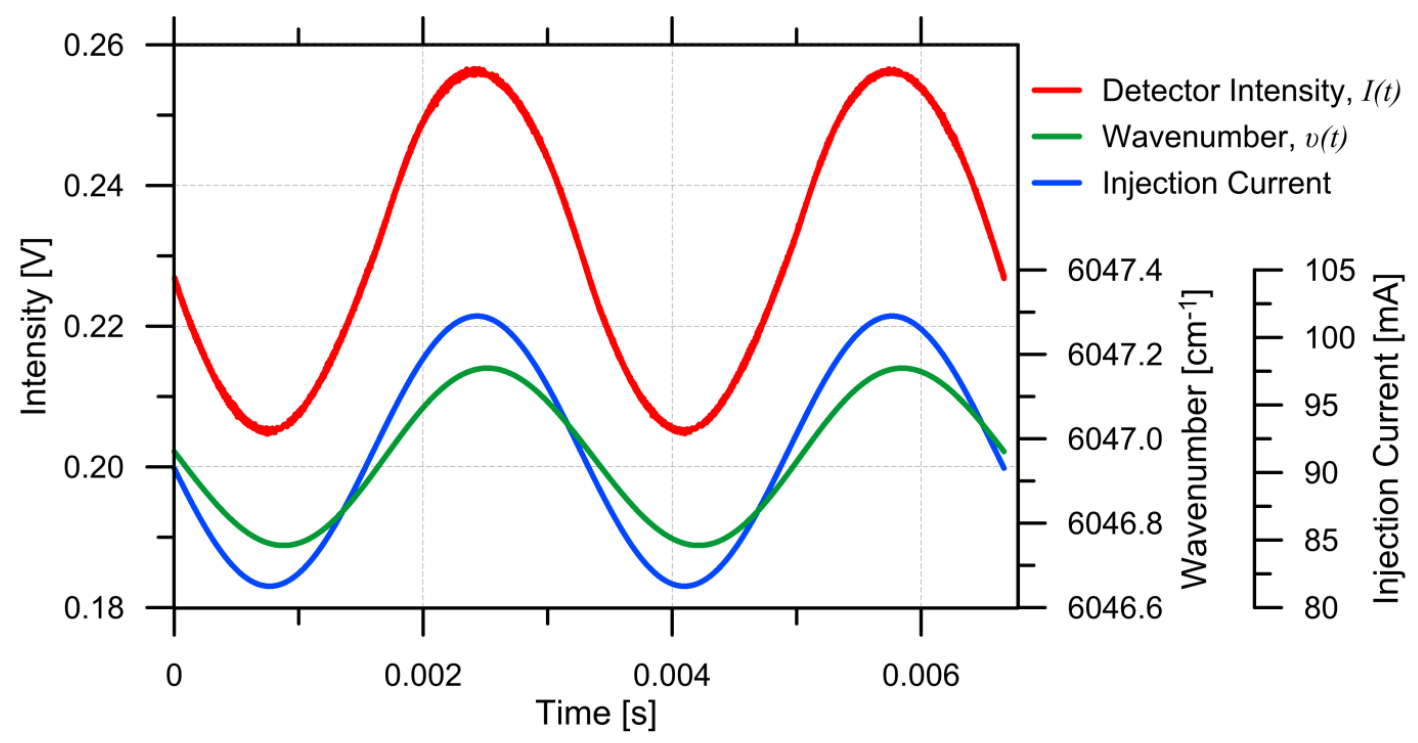

Figure 2.2: Measured intensity (red) and wavelength (green) SI signals resulting from an injection current sweep (blue)

After these signals are normalized by the average intensity, $\bar{I}_{0}$, the characteristic SI laser intensity and wavenumber parameters can be determined as illustrated in Figure 2.3. The reader is reminded to note the two sets of scales on both vertical axes necessary to visualize the much lower amplitude $2 f$ signals on the same plot as the $1 f$ signals.

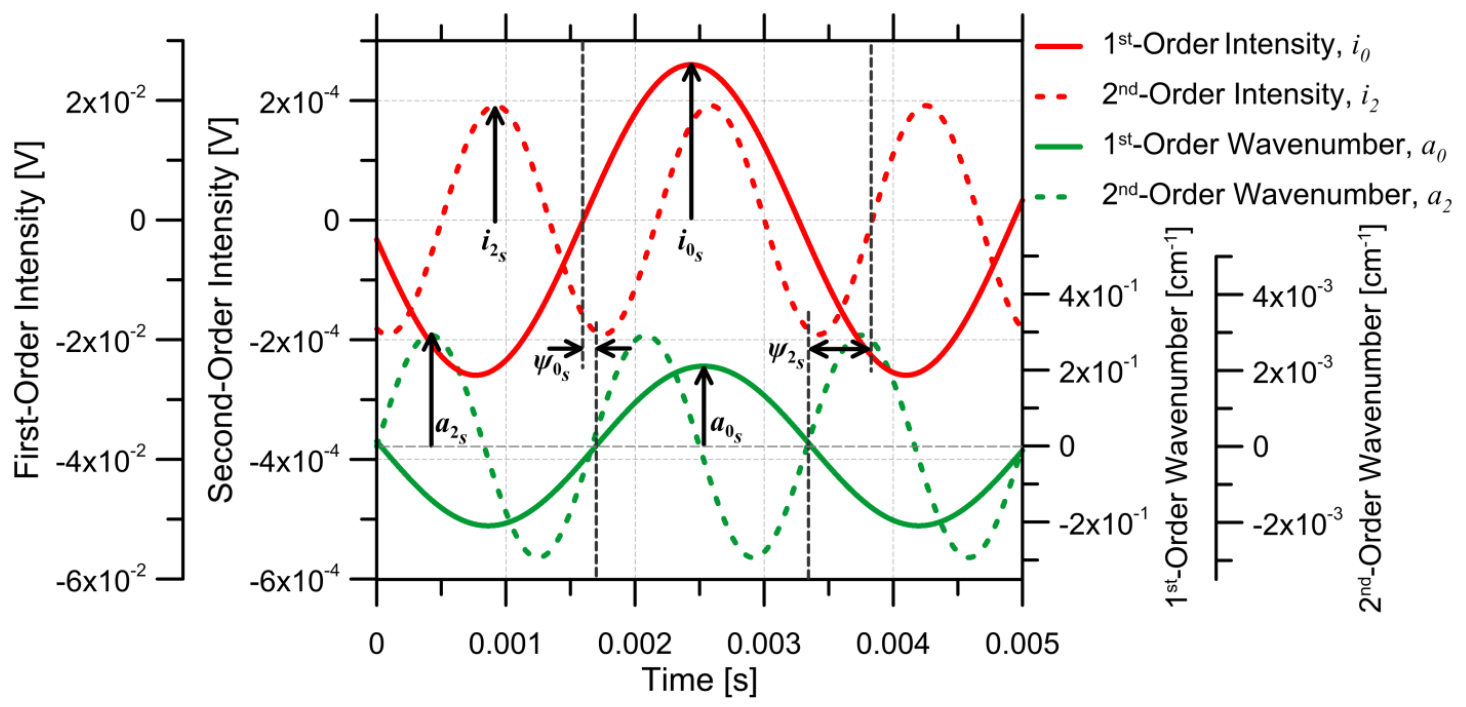

Figure 2.3: Simulated intensity (Red) and wavenumber (green) SI signal components and the corresponding laser characteristics (derived from experimental results) 
Some system design decisions, e.g. optimum SI operating conditions, were determined using simulated signals constructed with laser output characteristics as further detailed in Section 5.1. In addition, the transmittance signal, $\tau(t)$, can be modeled with simulated absorption-free signals, $I_{0_{S}}(t)$, following Eq. 2.1. Theoretically, the SI postabsorption intensity signal, $I_{S}(t)$, can be modeled by a product of the transmittance (Eq. 2.6, evaluated at the instantaneous optical frequency, $\left.v_{s}(t)\right)$ and the laser absorption-free intensity, $I_{0_{s}}(t)$, by:

$$
\begin{aligned}
I_{S}(t)=I_{0_{S}}(t) \tau & \left(v_{s}(t)\right) \\
& =I_{0_{S}}(t) \exp \left(-\sum_{i} \frac{q P x}{k T} \sigma_{v_{c_{i}}}\left(P, T, q, \bar{v}+a_{S} \cos \left(2 \pi f_{s} t\right)\right)\right)
\end{aligned}
$$

With Eq. 2.17, the methane volume mixing ratio (VMR) can be derived from the absorption, $\alpha(v)$ (Eq. 2.7), evaluated at the wavenumber of the absorption manifold peak, $v_{p}$, referred to as $x_{\mathrm{abs}}\left[\mathrm{ppm}_{\mathrm{v}}\right]$ :

$$
x_{\mathrm{abs}}=\alpha\left(v_{p}\right)=1-\frac{I_{s}\left(v_{p}\right)}{I_{0_{s}}\left(v_{p}\right)}
$$

Figure 2.4 illustrates a simulated absorption-free intensity signal (solid black), $I_{0_{S}}(t)$, and simulated (grey dash) and 1-second average (solid red) experimental SI intensity signals, $I_{S}(t)$, after absorption due to $200 \mathrm{ppm}_{\mathrm{v}}$ methane in nitrogen at ambient pressure and temperature. Simulated signals were calculated at $\bar{v}=1653.73 \mathrm{~nm}$, $a_{s} \approx 0.015 \mathrm{~nm}$, for a $5.79 \mathrm{~m}$ optical path-length, at near atmospheric temperature $\left(25^{\circ} \mathrm{C}\right)$ and pressure $(101.325 \mathrm{kPa})$. 


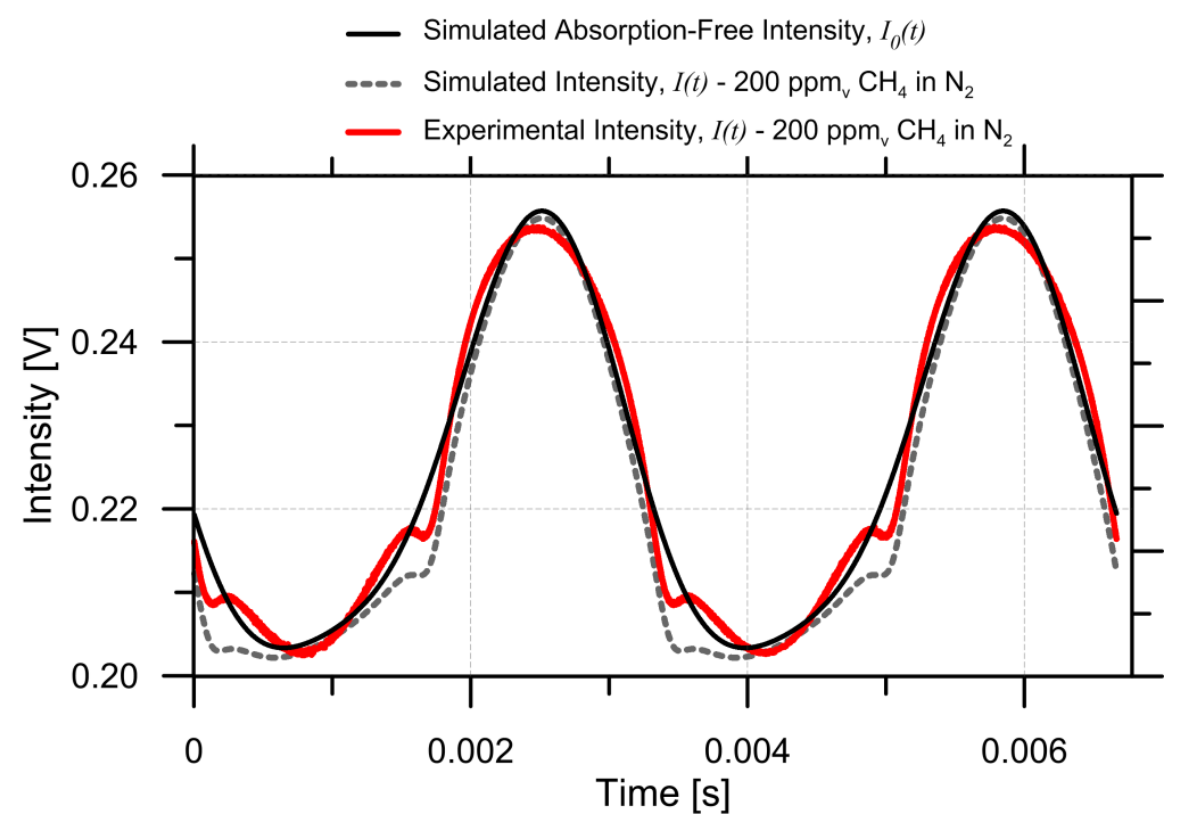

Figure 2.4: Simulated absorption-free return signal (black) and simulated and experimental (1-second average) SI intensity signals after absorption due to $200 \mathbf{p p m}_{\mathrm{v}}$ methane in nitrogen. Simulated signals were calculated at the experimental conditions (at ambient pressures and temperatures, and an optical path length of $5.79 \mathrm{~m})$, with $\bar{v}=1653.73 \mathrm{~nm}$ $\left(6046.94 \mathrm{~cm}^{-1}\right), a_{s} \approx 0.015 \mathrm{~nm}\left(\sim 0.009 \mathrm{~cm}^{-1}\right)$.

Figure 2.5 illustrates the resulting absorption signals, $\alpha(t)$, from the simulated and one-second averaged experimental SI intensity signals presented above. These results demonstrate discrepancies between the experimentally measured and theory-based absorption signals, which is attributable to improper estimation of the absorption free intensity as further discussed in Section 4.3.1. The relationship between the experimentally measured absorption intensity at the absorption manifold peak, $x_{\mathrm{abs}}=$ $\alpha\left(v_{p}\right)$, and the methane volume mixing ratio, $q$, is discussed in Section 4.6. 


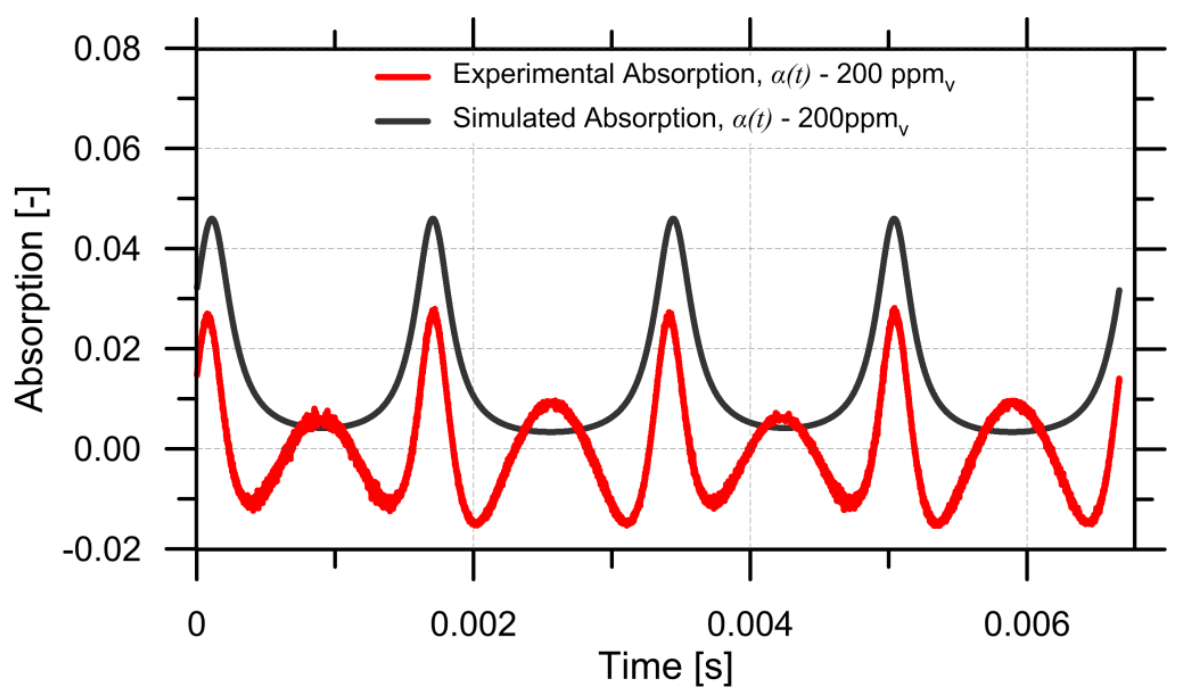

Figure 2.5: Simulated and experimental (1-second average) SI absorption signals with 200 $\mathbf{p p m}_{\mathrm{v}}$ methane in nitrogen. Simulated signals were calculated at the experimental conditions (at ambient pressures and temperatures, and an optical path length of $5.79 \mathrm{~m}$ ), with $\bar{v}=1653.73 \mathrm{~nm}\left(6046.94 \mathrm{~cm}^{-1}\right), a_{s} \approx 0.015 \mathrm{~nm}\left(\sim 0.009 \mathrm{~cm}^{-1}\right)$.

\subsubsection{Wavelength Modulation}

Similar to sweep integration, wavelength modulation (WM) tunes the laser output over a wavelength range using a modulated input current (Cassidy and Reid, 1982b; Bomse et al., 1992). However, in addition to the low frequency sweep used in the SI scheme, WM adds an additional high frequency sinusoid to the sweep signal, as demonstrated in Figure 2.6 .

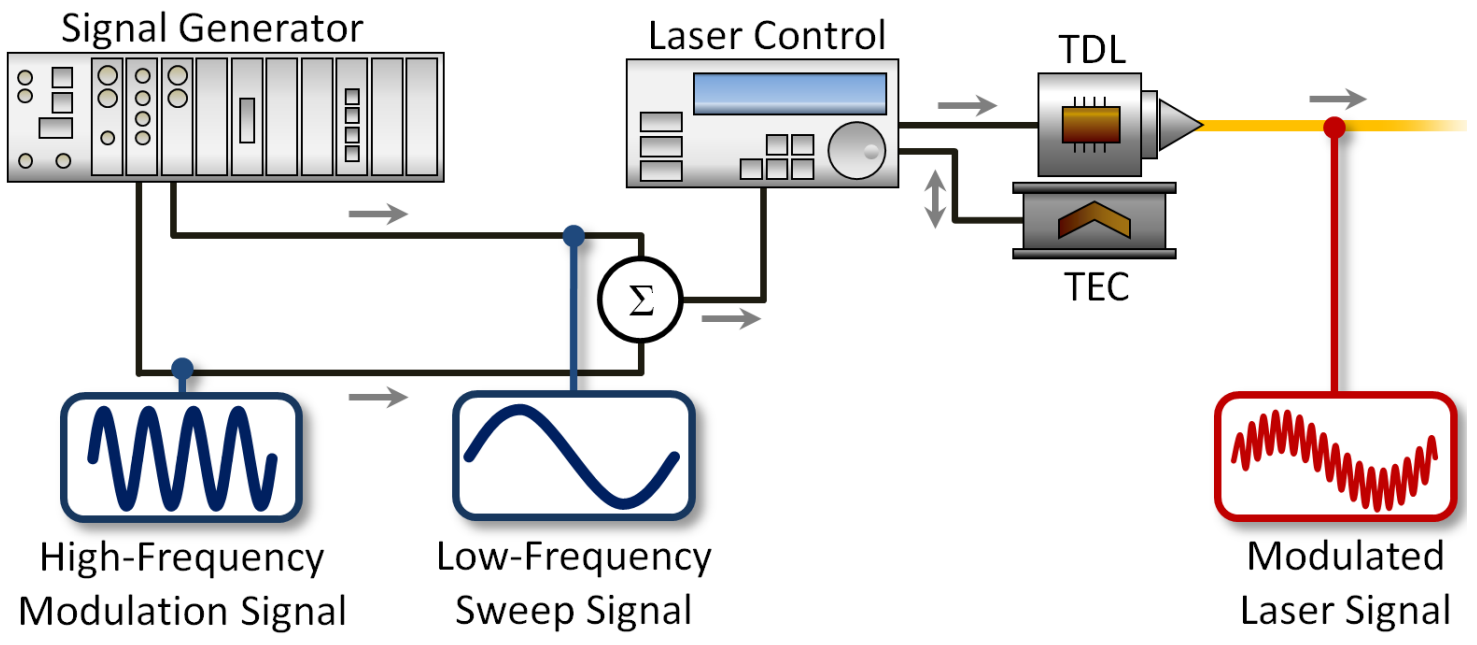

Figure 2.6: System schematic illustrating a summed low-frequency 'sweep' and highfrequency laser diode injection current modulation, resulting in a dual-frequency modulated output 
Similar to the sweep intensity and wavelength signals, the high frequency (modulated) laser intensity output signal, $I_{0_{m}}(t)$, can be well described by the first two harmonic terms, while the wavelength signal, $v_{m}(t)$, can be described by the first harmonic. These modulated signals can be described by:

$$
\begin{gathered}
I_{0_{m}}(t)=\bar{I}_{0}\left(1+i_{0_{m}} \cos \left(2 \pi f_{m} t+\psi_{1_{m}}\right)+i_{2_{m}} \cos \left(4 \pi f_{m} t+\psi_{2_{m}}\right)\right) \\
v_{m}(t)=\bar{v}+a_{m} \cos \left(2 \pi f_{m} t\right)
\end{gathered}
$$

where $f_{m}$ is the modulation frequency, $a_{m}$ is the spectral modulation depth $\left[\mathrm{cm}^{-1}\right]$, and $i_{0 m}, i_{2 m}$ are the first- and second-order modulation intensity amplitudes of the first- and second-order harmonics, at $f_{m}$ and $2 f_{m}$ respectively. Similar to the SI method, $\psi_{1_{m}}$ and $\psi_{2_{m}}$ account for the phase shifts between the first and second-order modulation intensity and wavelength signals. The complete WMS intensity and wavenumber signals can be derived by combining Eq. 2.19 and 2.20 with the equations describing the low-frequency sweep intensity and wavenumber (Eq. 2.15 and 2.16 respectively). From these, the instantaneous intensity signal $\left(I_{0_{\mathrm{WMS}}}(t)\right)$ can be described by the first two sweep and modulation harmonics, and the wavenumber signal $\left(v_{\mathrm{WMS}}(t)\right)$ can be described using only the first harmonics, following:

$$
\begin{gathered}
I_{0 \mathrm{WMS}}(t)=\bar{I}_{0}\left(1+i_{0_{s}} \cos \left(2 \pi f_{s} t+\psi_{1_{s}}\right)+i_{2_{s}} \cos \left(4 \pi f_{s} t+\psi_{2_{s}}\right)\right. \\
\left.+i_{0_{m}} \cos \left(2 \pi f_{m} t+\psi_{1_{m}}\right)+i_{2_{m}} \cos \left(4 \pi f_{m} t+\psi_{2_{m}}\right)\right) \\
v_{\mathrm{WMS}}(t)=\bar{v}++a_{s} \cos \left(2 \pi f_{s} t\right)+a_{m} \cos \left(2 \pi f_{m} t\right)
\end{gathered}
$$

Figure 2.7 demonstrates a typical absorption-free WMS experimental signal resulting from a low-frequency $1.5 \mathrm{~mA}$ sweep $(0.075 \mathrm{~V})$ and a high-frequency $\sim 20 \mathrm{~mA}$ $(1.0 \mathrm{~V})$ injection current modulation combined with a constant current offset of $95 \mathrm{~mA}$ at $\bar{v}=1653.73 \mathrm{~nm}$ (corresponding to the $\mathrm{R}(3)$ methane absorption manifold in the $2 v_{3}$ nearIR spectral branch). The reader is reminded to note that the solid colouring of the plot is due to the high-frequency of the modulation signal. The purpose of this plot is to illustrate the general form of these return signals, and provide a reference of the relative amplitudes and frequencies of the sweep and modulation signals. 


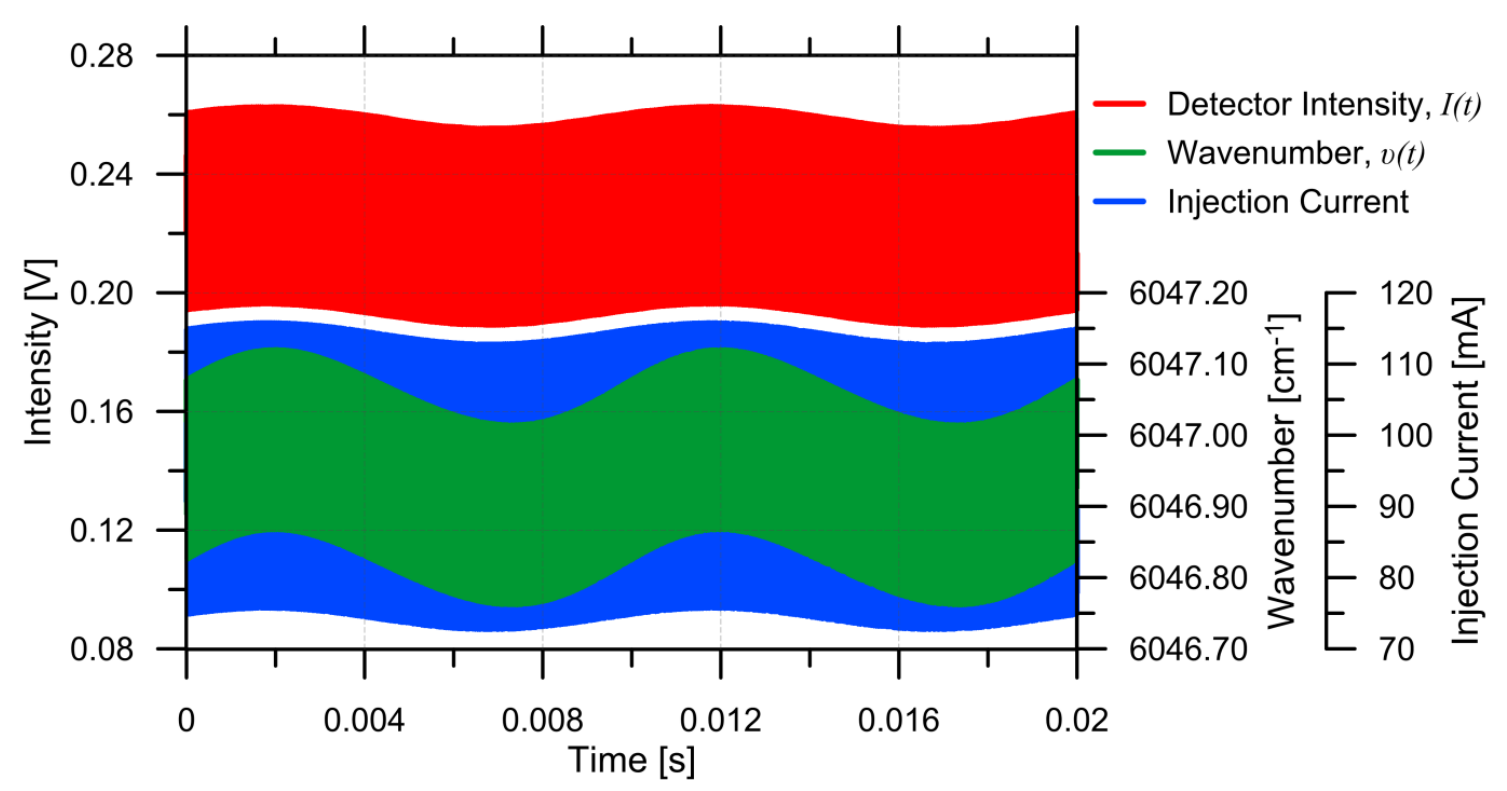

Figure 2.7: Raw WMS intensity absorption-free intensity (red) and wavelength (green) signals resulting from an injection current modulation (blue)

After these signals are normalized by the average intensity, $\overline{I_{0}}$, the characteristic WMS laser parameters can be determined. The resultant low-frequency intensity and wavelength components are as illustrated in Figure 2.3, whereas Figure 2.8 illustrates the high-frequency modulation components, separated into the first- (a) and second-order (b) signals at $f_{m}$ and $2 f_{m}$ respectively 
a) Simulated WMS Sweep Intensity and Wavelength

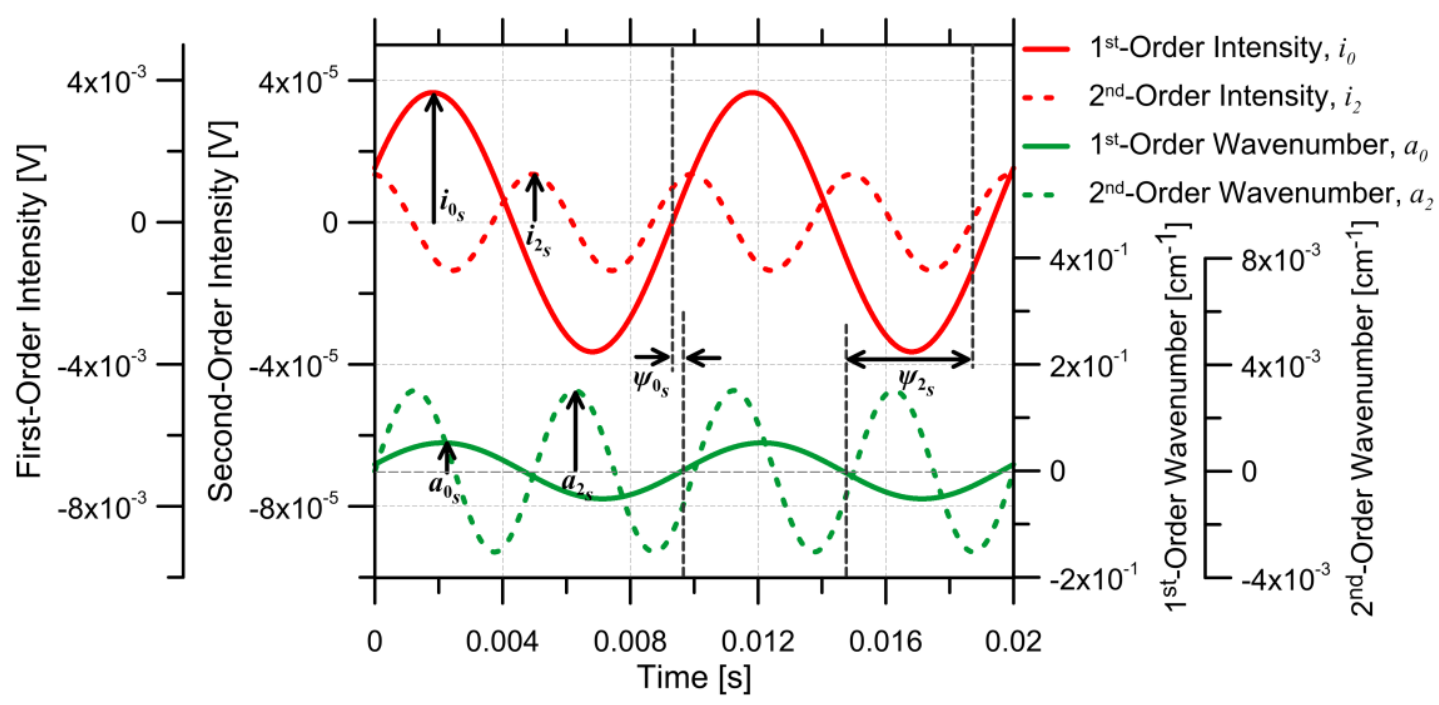

b) Simulated WMS Modulatation Intensity and Wavlength

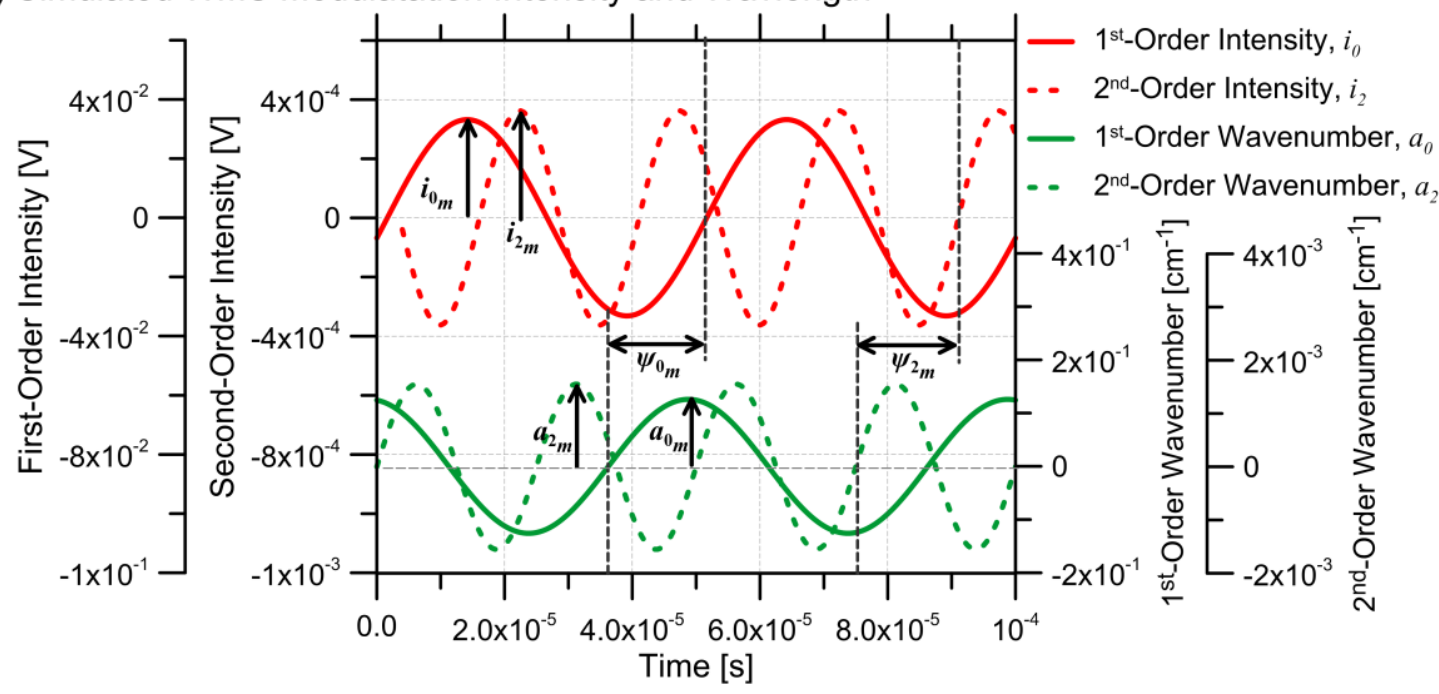

Figure 2.8: Simulated WMS characterization signals for the low- (a) and high-frequency (b) simulated absorption-free intensity (Red) and wavenumber (green), highlighting the corresponding laser characteristics

As with the SI method, some system design decisions were determined using simulated laser output characteristics, including the impact of residual amplitude modulation (Section 2.2.2) and optimum WMS operating conditions (Section 5.1). The post-absorption intensity signal, $I_{\mathrm{WMS}}(t)$, can be modeled by the Beer-Lambert-Bouguer Law (Eq. 2.1) with the laser pre-absorption intensity, $I_{0 \mathrm{WMS}}(t)$, evaluated at the instantaneous optical frequency, $v_{\mathrm{WMS}}(t)$, by: 


$$
\begin{aligned}
I_{\mathrm{WMS}}(t) & =I_{0 \mathrm{WMS}}(t) \tau\left(v_{\mathrm{WMS}}(t)\right) \\
& =I_{0 \mathrm{WMS}}(t) \exp \left(-\sum_{i} \frac{q P L}{k T} \sigma_{c_{c_{i}}}\left(P, T, q, \bar{v}+a_{s} \cos \left(2 \pi f_{s} t\right)+a_{m} \cos \left(2 \pi f_{m} t\right)\right)\right)
\end{aligned}
$$

To simulate the WMS signals, the transmittance can be written in terms of a Fourier series (with coefficients $H_{b}$ ) using reference to only the high-frequency terms (i.e. neglecting the sweep-frequency SI terms), following a similar nomenclature and procedure as found in ( $\mathrm{Li}$ et al., 2006; Rieker et al., 2009). To simplify notation, let $\eta=2 \pi f_{m} t$. The transmittance can be written as:

$$
\tau\left(v_{m}(\eta)\right)=\sum_{b=0}^{\infty} H_{b}\left(\bar{v}, a_{m}\right) \cos (b \eta)
$$

where the Fourier coefficients are defined by,

$$
\begin{aligned}
& H_{0}\left(\bar{v}, a_{m}\right)=\frac{1}{2 \pi} \int_{-\pi}^{\pi} \tau\left(\bar{v}+a_{m} \cos (\eta)\right) d \eta \\
& H_{b}\left(\bar{v}, a_{m}\right)=\frac{1}{\pi} \int_{-\pi}^{\pi} \tau\left(\bar{v}+a_{m} \cos (\eta)\right) \cos (b \eta) d \eta, \quad b \in \mathbb{N}
\end{aligned}
$$

The theory-based second harmonic ( $2 f)$ signal can be obtained by summing the even and odd second harmonic terms and computing the root-sum-square over each modulation period, $R_{2 f}=\sqrt{X_{2 f}^{2}+Y_{2 f}^{2}}$, where,

$$
\begin{aligned}
X_{2 f}=\frac{1}{2 \pi} \int_{-\pi}^{\pi} I_{0 m}(\eta) \tau\left(v_{m}(\eta)\right) \cos (2 \eta) \mathrm{d} \eta \\
\quad=\frac{\pi}{2}\left(H_{2}+\frac{i_{0_{m}}}{2}\left(H_{1}+H_{3}\right) \cos \left(\psi_{1 m}\right)+i_{2 m}\left(H_{0}+\frac{H_{4}}{2}\right) \cos \left(\psi_{2 m}\right)\right)
\end{aligned}
$$

and, 


$$
\begin{aligned}
Y_{2 f}=\frac{1}{2 \pi} \int_{-\pi}^{\pi} I_{0 m}(\eta) \tau\left(v_{m}(\eta)\right) \sin (2 \eta) \mathrm{d} \eta \\
\quad=-\frac{\overline{I_{0}}}{2}\left(\frac{i_{0 m}}{2}\left(H_{1}-H_{3}\right) \sin \left(\psi_{1_{m}}\right)+i_{2 m}\left(H_{0}-\frac{H_{4}}{2}\right) \sin \left(\psi_{2_{m}}\right)\right)
\end{aligned}
$$

In practice, $X_{2 f}$ and $Y_{2 f}$ were calculated over the $j^{\text {th }}$ modulation period with a Riemann sum approximation, using the measured intensities at each sample time-index, $t_{i}$, following:

$$
\begin{aligned}
& X_{2 f}(j)=\frac{1}{M} \sum_{i=0}^{M} I_{\mathrm{WMS}}\left(t_{i}\right) \cos \left(4 \pi f_{m} t_{i}\right) \\
& Y_{2 f}(j)=\frac{1}{M} \sum_{i=0}^{M} I_{\mathrm{WMS}}\left(t_{i}\right) \sin \left(4 \pi f_{m} t_{i}\right)
\end{aligned}
$$

where $M$ is total number of samples in a modulation period $\left(M=\right.$ sample frequency $\left./ f_{m}\right)$. The time varying $2 f$ signal is defined by assigning the center time $t_{j}$ of each $j^{\text {th }}$ modulation period to $X_{2 f}(j)$ and $Y_{2 f}(j)$. The $2 f$ signal is computed following:

$$
R_{2 f}\left(t_{j}\right)=\sqrt{X_{2 f}\left(t_{j}\right)^{2}+Y_{2 f}\left(t_{j}\right)^{2}}
$$

Figure 2.9 illustrates simulated and experimental (1-second average) WMS intensity signals generated with $200 \mathrm{ppm}_{\mathrm{v}}$ methane in nitrogen (black and red lines respectively), acquired at ambient pressure and temperature. The simulated signals were calculated at $\bar{v}=1653.73 \mathrm{~nm}, a_{s} \approx 0.015 \mathrm{~nm}, T=23^{\circ} \mathrm{C}, p=101.325 \mathrm{kPa}$, and using a $5.79 \mathrm{~m}$ optical path-length. The zoomed view on the right side of the figure illustrates the difference between these signals. 

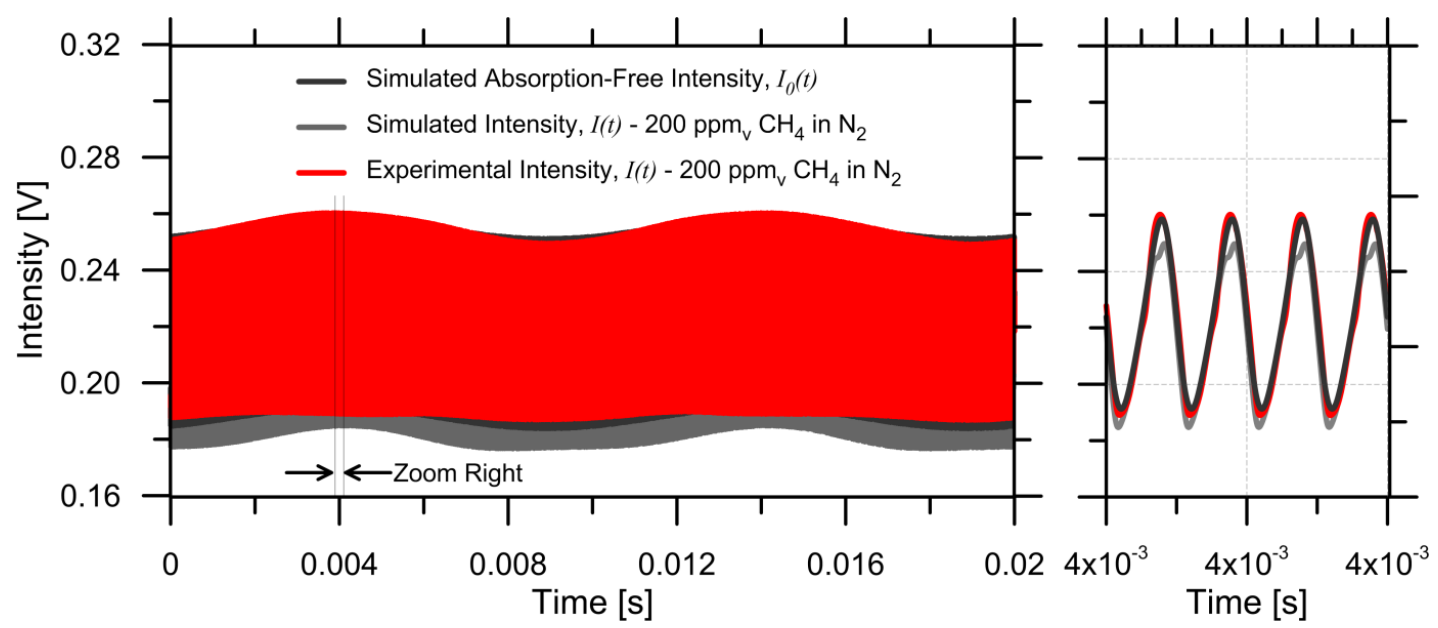

Figure 2.9: Simulated absorption-free intensity (black), and simulated (grey) and experimental (red) WMS intensity signals generated with $200 \mathrm{ppm}_{\mathrm{v}}$ methane in nitrogen, calculated using an optical path-length of $5.79 \mathrm{~m}$, temperature of $23^{\circ} \mathrm{C}$, and pressure of

$101.325 \mathrm{kPa}$, with $\bar{v}=1653.73 \mathrm{~nm}\left(6046.94 \mathrm{~cm}^{-1}\right), a_{s} \approx 0.015 \mathrm{~nm}\left(\sim 0.009 \mathrm{~cm}^{-1}\right)$, and ,

$$
a_{m} \approx 0.032 \mathrm{~nm}\left(\sim 0.020 \mathrm{~cm}^{-1}\right) \text {. }
$$

Figure 2.10 illustrates the simulated and experimental second-harmonic (2f) WMS signals, $R_{2 f}\left(t_{c}\right)$, resulting from the WMS intensity signals presented above.

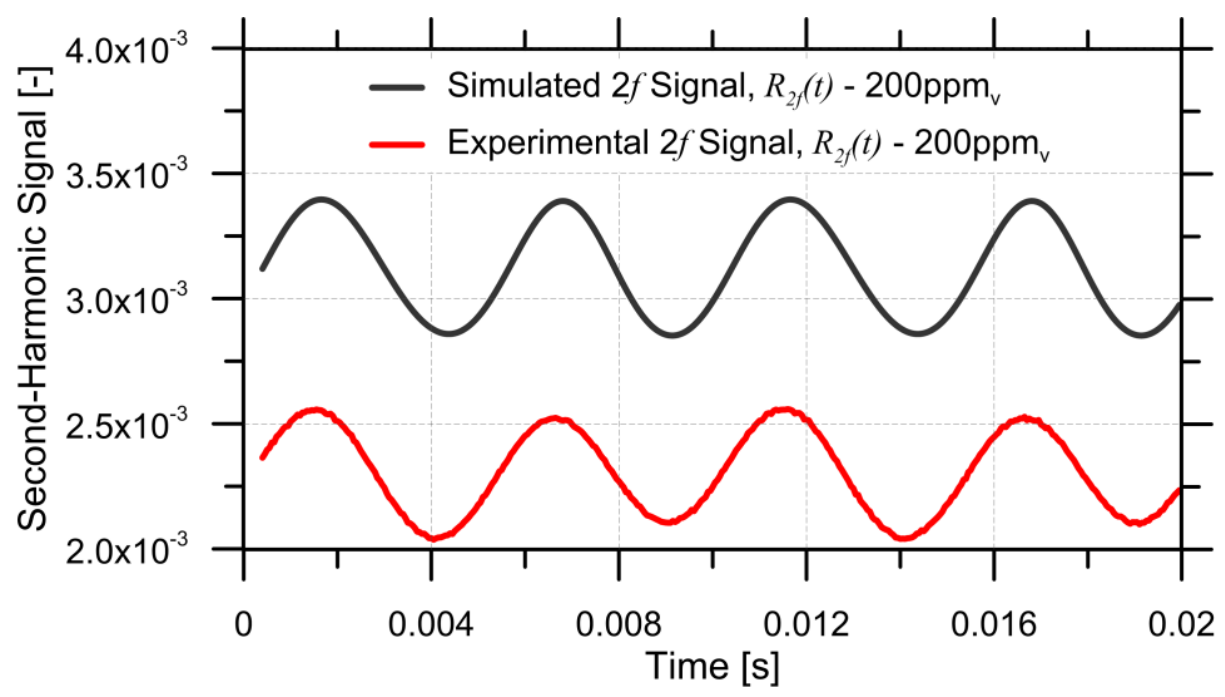

Figure 2.10: Simulated and experimental (1-second average) WMS $2 f$ signals with $200 \mathrm{ppm}_{\mathrm{v}}$ methane in nitrogen. Simulated signals were calculated at the experimental conditions (at ambient pressures and temperatures, and an optical path length of $5.79 \mathrm{~m}$ ), with $\bar{v}$ $=1653.73 \mathrm{~nm}\left(6046.94 \mathrm{~cm}^{-1}\right), a_{s} \approx 0.015 \mathrm{~nm}\left(\sim 0.009 \mathrm{~cm}^{-1}\right)$, and,$a_{m} \approx 0.032 \mathrm{~nm}\left(\sim 0.020 \mathrm{~cm}^{-1}\right)$.

These results demonstrate that there are discrepancies between the theory-based and experimental $2 f$ WMS signals, as further discussed in Section 4.3. The relationship 
between the experimentally measured second-harmonic height at the $2 f$ peak, $x_{2 f}=$ $R_{2 f}\left(v_{p}\right)$, and the methane volume mixing ratio, $q$, is discussed in Section 4.4.

\subsubsection{Residual Amplitude Modulation}

The desired result of modulating the tunable diode laser injection current is to vary the output wavelength. However, the laser output wavelength and power are coupled, resulting in an additional simultaneous laser intensity residual amplitude modulation (RAM). Although repeatable, this signal can be difficult to suppress or remove since it occurs at the same frequency as the driving signals. Furthermore, since RAM is a background signal, it introduces a constant bias error, which is increasingly influential on measurement error at low absorption intensities.

The effect of RAM has been a major topic of discussion in TDLAS literature, with considerable focus on methods of suppression or removal (Gehrtz et al., 1985; Linnerud et al., 1998; Kluczynski et al., 2001; Liu et al., 2004b; De Tommasi et al., 2007; Duffin et al., 2007; Chakraborty et al., 2010). To estimate the influence of RAM on the theory-based WMS approximations, the laser intensity with and without RAM after methane absorption was simulated.

As discussed previously, the output intensity $I_{0_{W M S}}(t)$ and wavelength $v_{W M S}(t)$ can be described by Eq. 2.21 and 2.22. Combined with measured laser characteristics, the post absorption intensity, $I_{W M S}(t)$, can be described by Eq. 2.23. RAM subtraction refers to removing intensity modulation which is unaccounted for in the theory-based $2 f$ WMS approximation, $R_{2 f}$. By making the assumption that transmittance is small, the intensity due to the sweep (Equation 2.15) can be used to approximate the RAM signal, $I_{\text {RAM }}(t)$. This can then be subtracted from post-absorption signals, $I_{\mathrm{WMS}}(t)$, leaving only the post-absorption intensity signal introduced by the high-frequency modulation, $I_{m}(t)$. Figure 2.11 illustrates simulated WMS signals, swept over the near-IR $2 v_{3} R(3)$ methane absorption manifold (See Section 2.3) with a sweep $\left(a_{s} \approx 0.009 \mathrm{~nm}\right.$, Red), without sweep (Black dashed), and with the sweep subtracted (Blue). Calculations were performed for 
methane concentrations of 2 and $200 \mathrm{ppm}_{\mathrm{v}}$ in nitrogen, using an optical path-length of $5.79 \mathrm{~m}$, at $23^{\circ} \mathrm{C}$ and $101.325 \mathrm{kPa}$.

a) Simulated $2 f$ Signal - $2 \mathrm{ppm}_{\mathrm{v}}$ Methane in Nitrogen

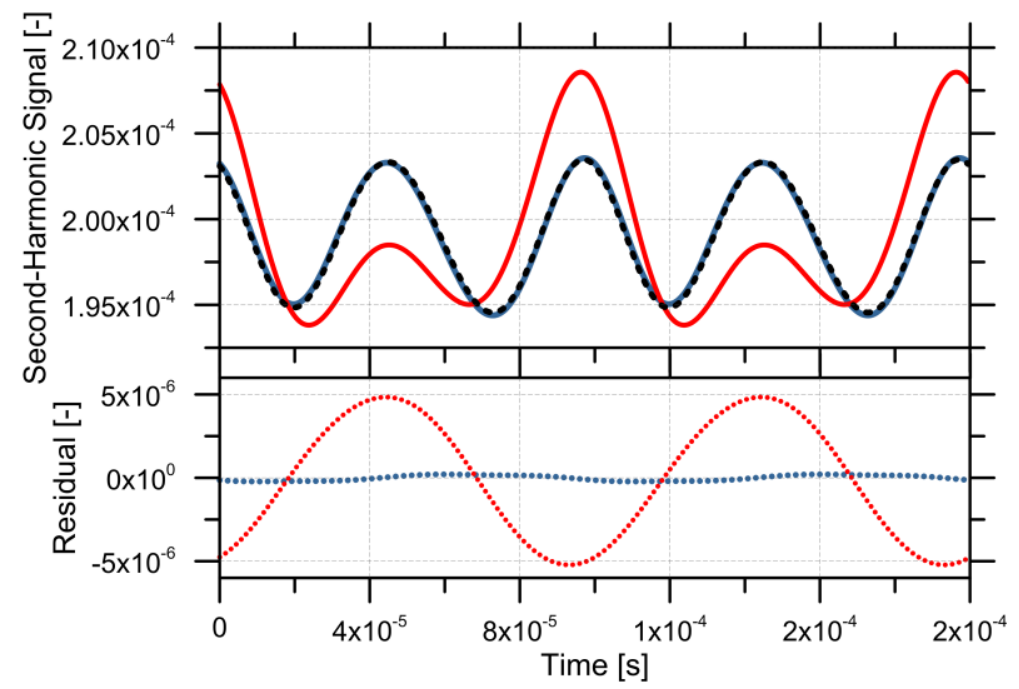

b) Simulated $2 f$ Signal $-200 \mathrm{ppm}_{\mathrm{v}}$ Methane in Nitrogen

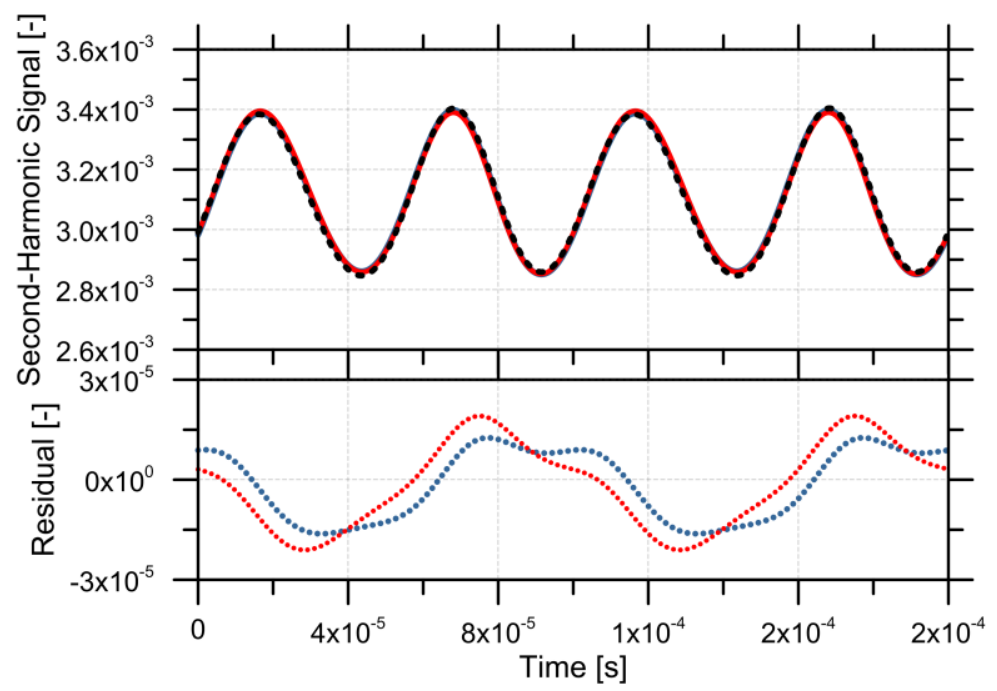

$$
\begin{aligned}
& \text {... (0) Simulated } 2 f \text { without Sweep } \quad \text {...... Residual } 2 f(0) \text {-(II) } \\
& \text { — (I) Simulated } 2 f \text { Signal } \quad \ldots \quad \text { Residual } 2 f(0)-(\mathrm{I}) \\
& \text { - (II) Simulated } 2 f \text { with RAM Subtracted }
\end{aligned}
$$

Figure 2.11: Simulated WMS $2 f$ Signals and Residuals with and without RAM Subtraction: (a) $2 \mathrm{ppm}_{\mathrm{v}}$, (b) $200 \mathrm{ppm}_{\mathrm{v}}$ with an optical path-length of $5.79 \mathrm{~m}$, at $23^{\circ} \mathrm{C}$ and $101.325 \mathrm{kPa}$.

For the simulated concentrations, the signals without the sweep (Black dashed) and with the RAM subtracted (Blue) conform very well, even after absorption. Figure 2.12 demonstrates the difference between a simulated WMS signal without a sweep over 
the maximum of the $2 v_{3} \mathrm{R}(3)$ methane absorption manifold and signals with and without RAM subtraction, for methane volume mixing ratios between 0.5 and $450 \mathrm{ppm}_{\mathrm{v}}$.

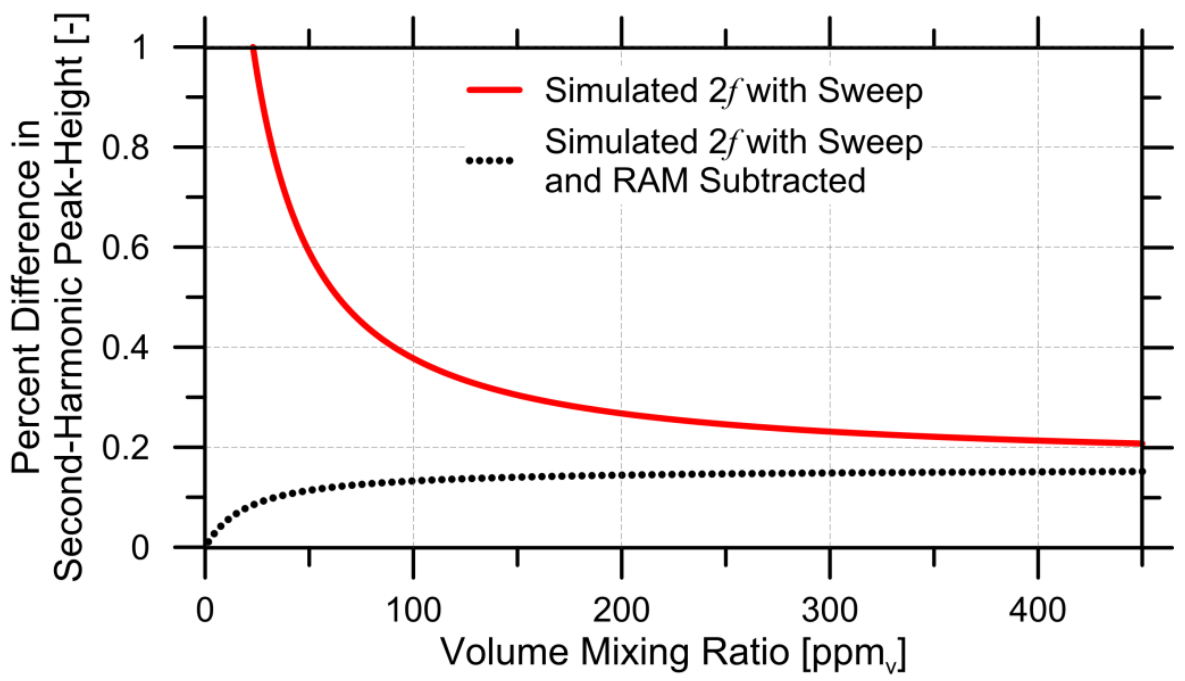

Figure 2.12: Percent-difference of simulated $2 f$ values obtained at the $2 v_{3} R(3)$ methane absorption manifold with and without RAM-subtraction, evaluated against the ideal WMS signal without RAM (no sweep) using an optical path-length of $5.79 \mathrm{~m}$, at $23^{\circ} \mathrm{C}$ and 101.325 kPa.

The simulated $2 f$ value obtained with RAM subtraction conforms better to the theoretical values over the intended measurement range of this system. In addition, this simulation suggests that RAM subtraction may be increasingly beneficial with decreasing volume mixing ratios, and may not have as significant of an impact at volume mixing ratios above $500 \mathrm{ppm}_{\mathrm{v}}$. Figure 2.13 illustrates the difference between the measured methane volume mixing ratio (derived using Equations 2.27 and 2.28, using an optical path-length of $5.79 \mathrm{~m}$, at $23^{\circ} \mathrm{C}$ and $101.325 \mathrm{kPa}$ ) for the simulated $2 f$ signals with and without RAM subtraction. 


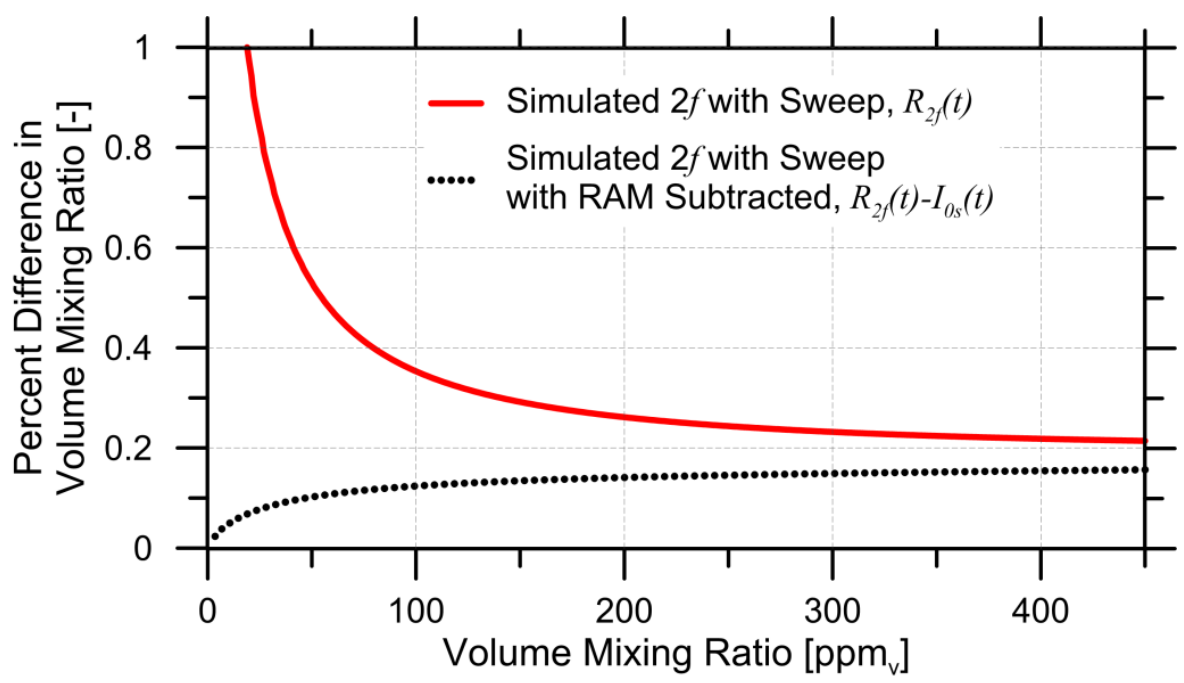

Figure 2.13: Percent-difference of simulated methane volume mixing ratio at the $2 v_{3} R(3)$ methane manifold with and without RAM-subtraction, evaluated against the ideal WMS signal without RAM (no sweep) using an optical path-length of $5.79 \mathrm{~m}$, at $23^{\circ} \mathrm{C}$ and 101.325 kPa.

These simulated results illustrate that low-frequency RAM can be subtracted from $2 f$ signals post-absorption to better conform to theoretical simulations. The potential implications of these results, including further discussions on the influence of RAM suppression on methane VMR measurements, are discussed in Section 5.3.2.

\subsection{Absorption-peak Selection}

To ensure the best system measurement performance, a strong, well-defined, and isolated methane absorption feature is necessary. For methane, the strongest absorption lines are found within the mid-IR range ( $v_{3}$ band around $3.3 \mu \mathrm{m}$ ). Even though quantum cascade lasers (QCLs) and fiber-based optical waveguides have made sensing in this spectral region more accessible (Sigrist et al., 2007; Lackner, 2007; Song and Jung, 2003; Kosterev et al., 2007), their cost and availability has not yet reached the breadth of inexpensive commercial and custom components available in the near-IR region, driven by the large markets of the telecommunications industry.

Fortunately there are methane absorption combinations and overtones within the near-IR spectral operating ranges of widely-available, low-cost custom-wavelength lasers and detectors developed for the telecommunications industry. Although not as strong as 
mid-IR absorption bands, detectors used within the near-IR region tend to have much higher shot noise levels if they are not cryogenically cooled, limiting the sensitivity of measurements to be on the order of what is achievable with a near-IR setup (Stewart et al., 1998b). In the near-IR, methane absorption lines are grouped into two spectral bands: a combination band $\left(v_{2}+2 v_{3}\right)$ around $1.33 \mu \mathrm{m}$, and a harmonic band $\left(2 v_{3}\right)$ around $1.66 \mu \mathrm{m}$ (Rothman et al., 2009). Figure 2.14 illustrates these two methane absorption bands (vertical grey shaded regions), the operational wavelengths of common semiconducting detectors (red), and the theoretical losses of standard 9/125 $\mu \mathrm{m}$ telecommunications-grade silica single-mode fiber (black) optics in near-IR spectrum.

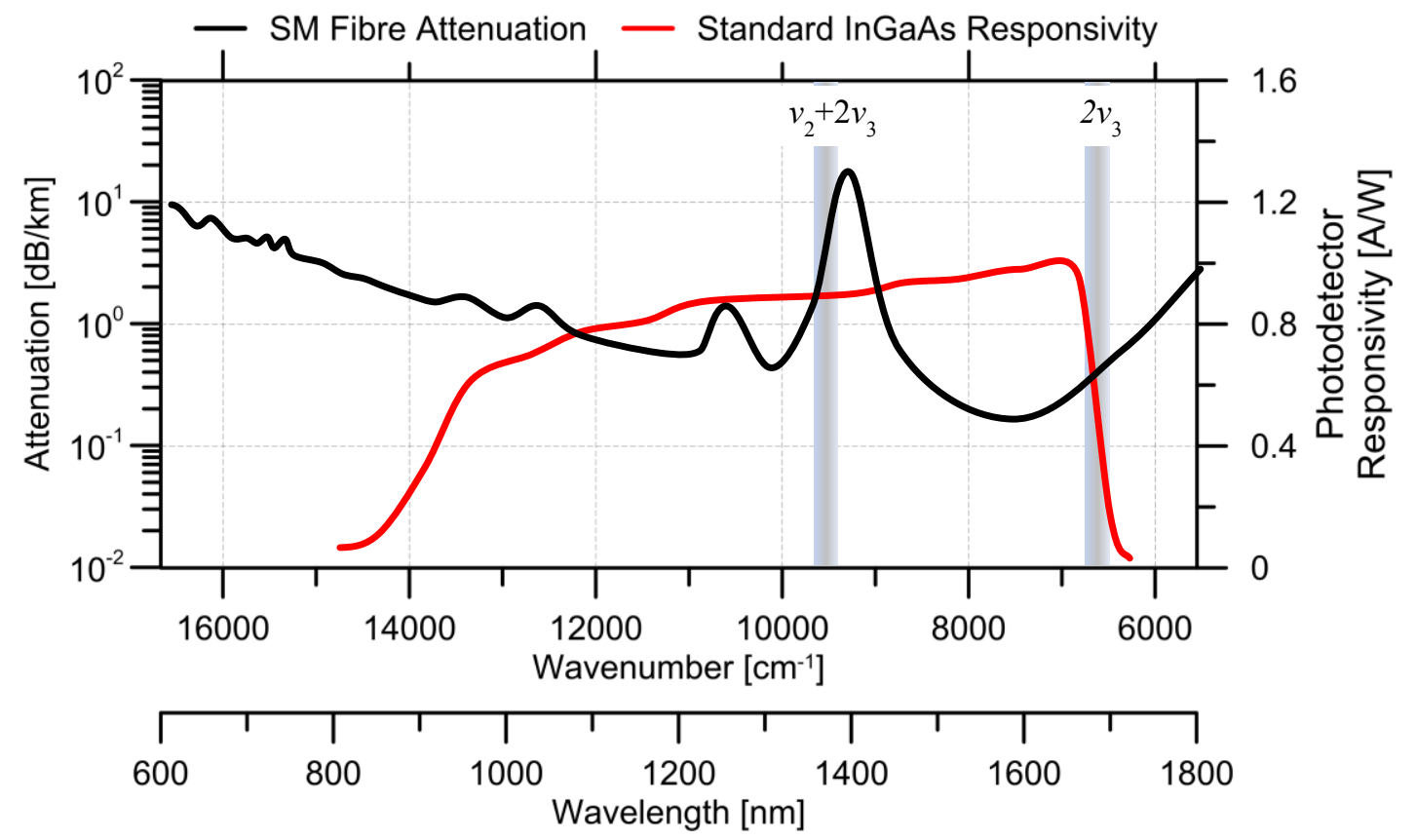

Figure 2.14: Comparison of methane spectral absorption bands (grey shaded regions) to standard 9/125 $\mu \mathrm{m}$ telecommunications fiber optic attenuation and semiconductor detector responsivity in the Near-IR. Adapted from (Schubert, 2003; Roberson and Cook, 2011).

Absorption peaks around $1.66 \mu \mathrm{m}$ are stronger and broader than at $1.33 \mu \mathrm{m}$ since they are a harmonic rather than a combinational band. Within these bands the rotational and vibrational states can be spectrally organized in to the P, Q, and R branches (as labelled in Figure 2.16, where the P-branch is to the right of the plotted data); relating to the ground state, $\mathrm{Q}$ (purely rotational), and ro-vibration combined states, $\mathrm{P}$ and $\mathrm{R}$ (on low 
and high wavenumber sides of Q branch respectively). The spectral lines of significant atmospheric absorbers within this spectral region are illustrated in Figure 2.15.

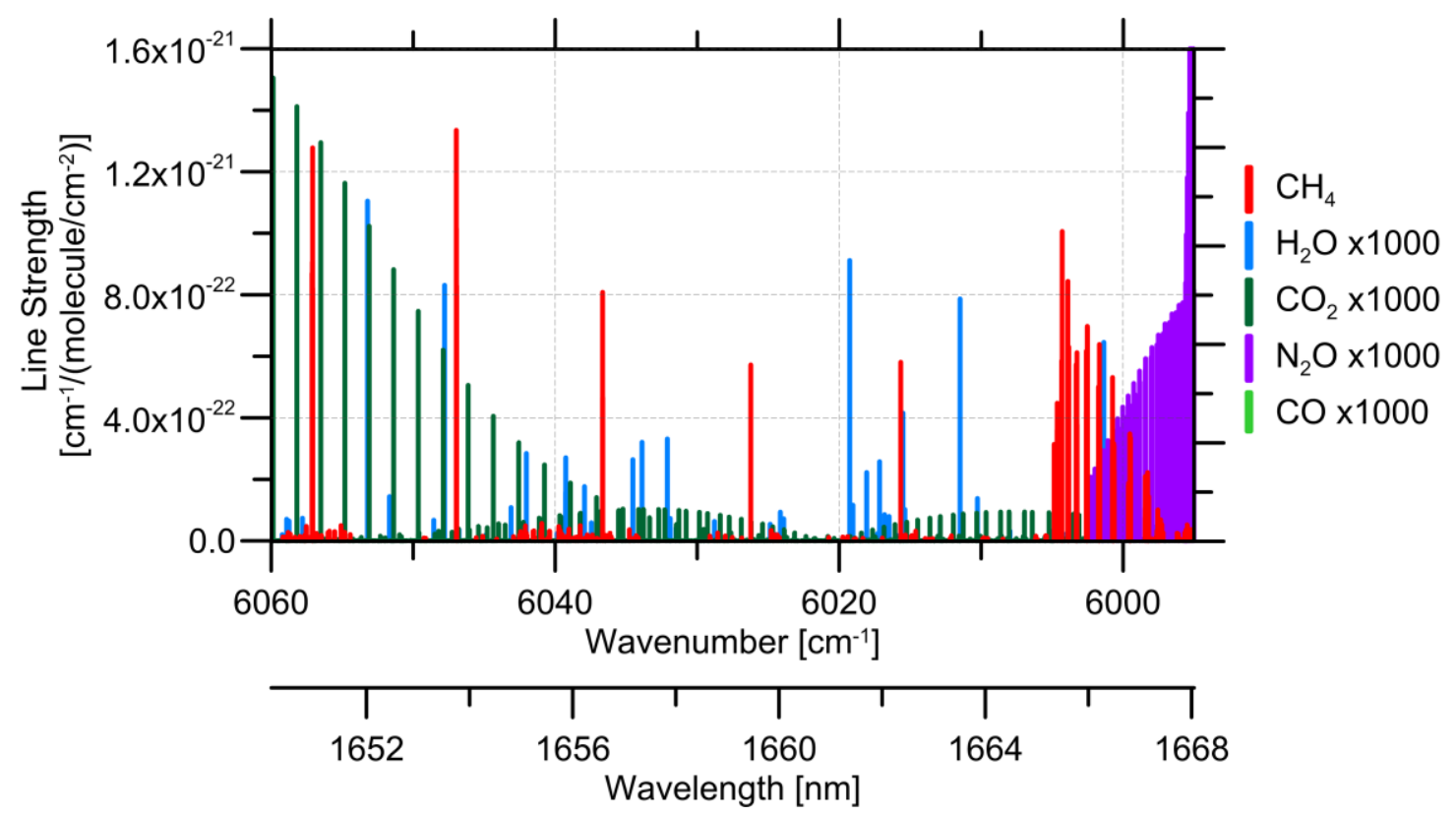

Figure 2.15: Spectral lines of significant atmospheric absorbers within this spectral region. Data from HITRAN2012 (Rothman et al., 2013)

Note that in Figure 2.15 the line strength for the spectral lines other than methane are multiplied by a factor of 1000 for plotting purposes, which further demonstrates how the line strengths for methane are significantly more pronounced than those of the next top 4 absorbers. However, VMR must be taken into account for a complete comparison. Figure 2.16 illustrates the absorption for the top five most significant common atmospheric absorbers at their nominal atmospheric concentrations, within the same $1.66 \mu \mathrm{m} 2 v_{3}$ band, calculated using a Lorentz profile at $20^{\circ} \mathrm{C}$ and $101.325 \mathrm{kPa}$, using a $5.79 \mathrm{~m}$ optical path-length. 


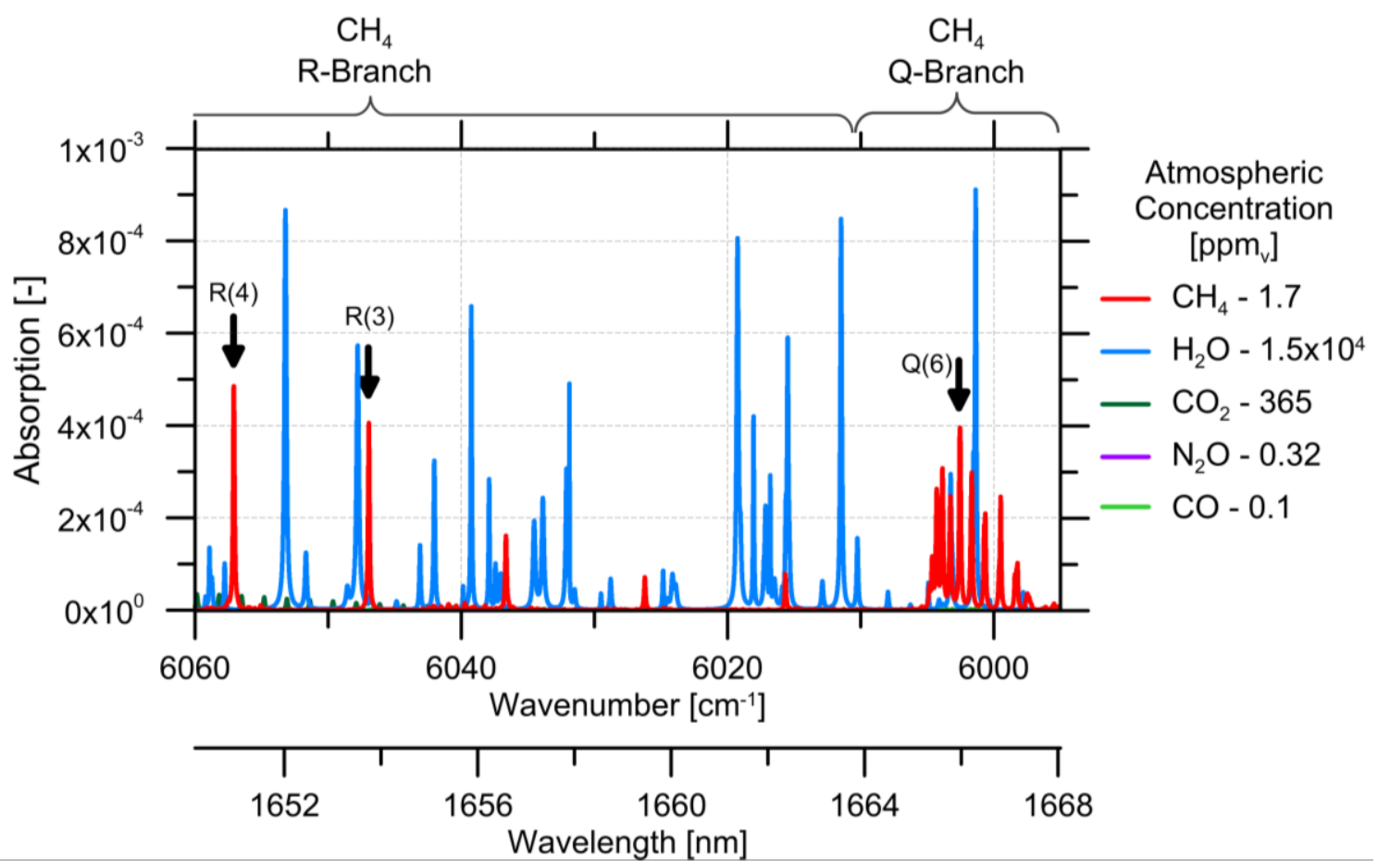

Figure 2.16: Absorption strength of near-IR $2 v_{3} Q$ and $R$ methane absorption bands; including prominent atmospheric absorbers for reference. Calculated using a Lorentz profile at $20^{\circ} \mathrm{C}$ and $101.325 \mathrm{kPa}$, using a $5.79 \mathrm{~m}$ optical path-length.

At atmospheric pressures, temperatures, and concentrations, methane is a considerable absorber of light between 1650 and $1670 \mathrm{~nm}$, second only to water. There are methane absorption manifolds which overlap with water and must be avoided to prevent interference with a methane VMR estimate. In addition to being isolated from other atmospheric absorbers, to facilitate accurate measurement, the selected methane absorption manifold must also be isolated from adjacent methane manifolds, must fall within the working range of available tunable diode lasers (TDLs), must be well defined by the HITRAN database (Rothman et al., 2009), and must have a somewhat symmetric shape governed by the close arrangement of absorption lines within the manifold. Methane manifolds which meet these criteria are $\mathrm{R}(3), \mathrm{R}(4)$, and $\mathrm{Q}(6)$. Water at the maximum expected atmospheric VMR of $15,000 \mathrm{ppm}_{\mathrm{v}}$ adds only $0.30 \%, 3.57 \%$, and $1.61 \%$ to the methane $\mathrm{R}(3), \mathrm{R}(4)$, and $\mathrm{Q}(6)$ absorption manifolds at atmospheric pressure, temperature, and a VMR of $1.7 \mathrm{ppm}_{\mathrm{v}}$. This minimal influence decreases significantly at higher methane VMRs. In addition, these manifolds each have a point within their 
immediate spectral region that has particularly low absorption from atmospheric absorbers (including methane), which is useful for reference purposes.

The theoretical absorption at any wavelength requires detailed information of the influential absorbers within the spectral region. However, without simplification, an absorption calculation including all absorption lines from relevant molecules (and isotopes) in the HITRAN database is inefficient and arguably unfeasible. Therefore, a cut-off criterion was used to determine the minimum number of relevant lines on either side of a center wavelength to include. The relevance of contributing absorption lines to the theory-based absorption was determined by finding the minimum number of methane lines from the HITRAN2008 database which incurred a maximum error on the absorption peak height at $6002.54 \mathrm{~cm}^{-1}$, corresponding to less than a $0.001 \%$ change relative to using all 290091 lines in the database (Tyner, 2015; Rothman et al., 1998). This calculation was performed using a Lorentzian absorption profile at 10,000 $\mathrm{ppm}_{\mathrm{v}}$ methane in nitrogen at $T=20^{\circ} \mathrm{C}$ and $p=101.325 \mathrm{kPa}$, using the two most abundant isotopes of methane on earth. Figure 2.17 illustrates the combined theoretical absorption profile and the methane absorption lines for the $\mathrm{R}(3), \mathrm{R}(4)$, and $\mathrm{Q}(6)$ manifolds, calculated at $1.7 \mathrm{ppm}_{\mathrm{v}}$ methane in nitrogen at ambient pressure and temperature for an optical pathlength of $5.79 \mathrm{~m}$. 


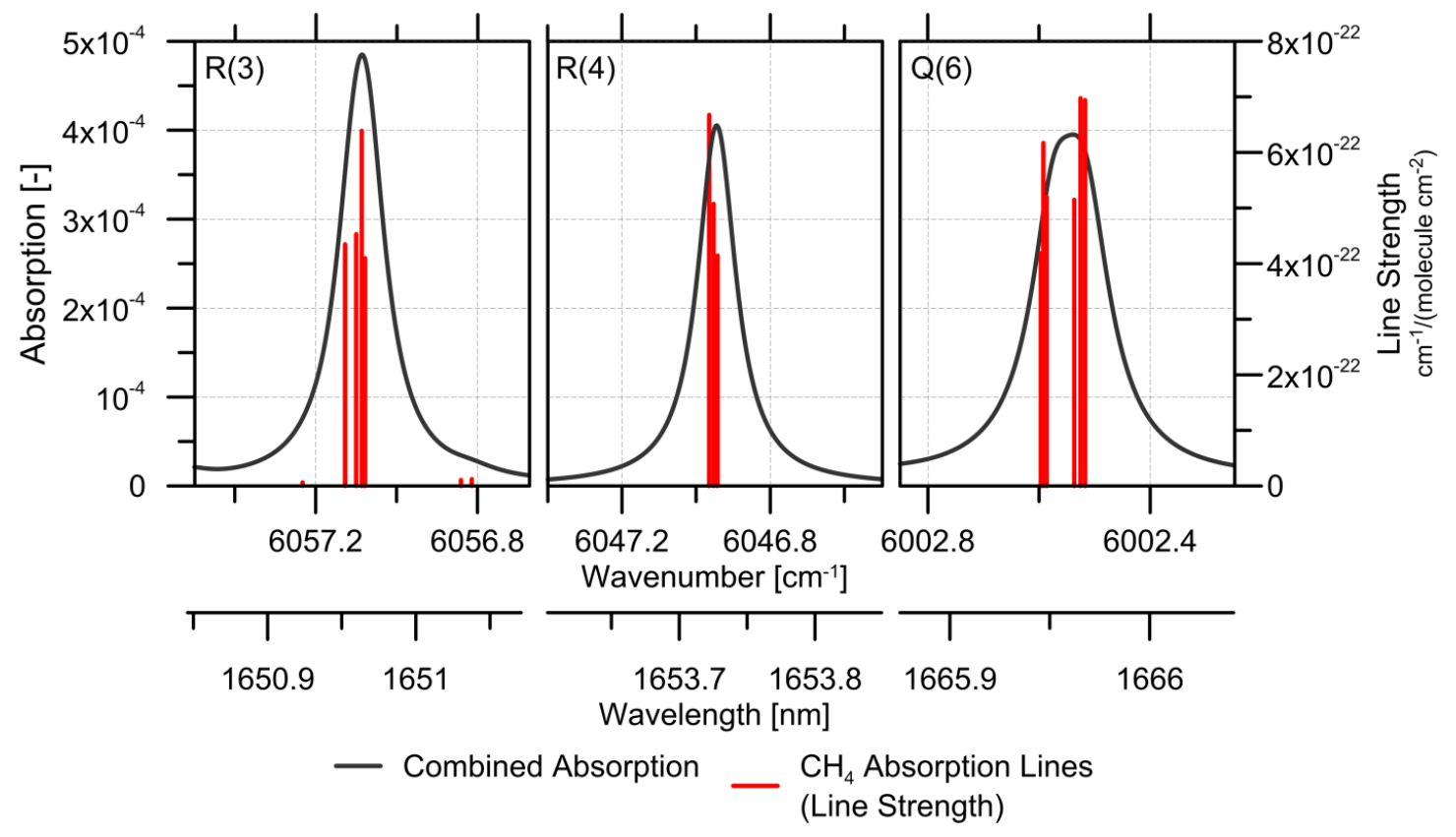

Figure 2.17: Combined absorption of all significant standard atmospheric absorbers (i.e. $1.7 \mathrm{ppm}_{\mathrm{v}} \mathrm{CH}_{4}, 15,000 \mathrm{ppm}_{\mathrm{v}} \mathrm{H}_{2} \mathrm{O}, 365 \mathrm{ppm}_{\mathrm{v}} \mathrm{CO}_{2}, 0.32 \mathrm{ppm}_{\mathrm{v}} \mathrm{N}_{2} \mathrm{O}$, and $0.1 \mathrm{ppm}_{\mathrm{v}} \mathrm{CO}$ ) around the $R(3), R(4)$, and $Q(6)$ methane manifolds. Plots were calculated at $20^{\circ} \mathrm{C}$ and $101.325 \mathrm{kPa}$, using a $5.79 \mathrm{~m}$ optical path-length.

Characteristics of the selected methane manifolds of interest, such as center wavenumber, half-width at half-maximum (HWHM), and the number of relevant absorption lines used for theoretical computation are listed in Table 2.4.

Table 2.4: Relevant Methane Manifolds in the near-IR

\begin{tabular}{|c|c|c|c|c|}
\hline \multirow[b]{2}{*}{ Parameter } & \multirow[b]{2}{*}{ Units } & \multicolumn{3}{|c|}{ Methane $2 v_{3}$ Manifold } \\
\hline & & $\mathbf{R}(3)$ & $R(4)$ & $Q(6)$ \\
\hline Wavenumber of manifold max., $v_{p}$ & $\mathrm{~cm}^{-1}$ & 6046.945 & 6057.087 & 6002.544 \\
\hline Half-width at half-maximum & $\mathrm{cm}^{-1}$ & 0.068 & 0.070 & 0.077 \\
\hline Number of Absorption Lines Included & \# & 530 & 545 & 626 \\
\hline Absorption $+\left[\times 10^{-4}\right]$ & {$[-]$} & 4.47 & 3.67 & 3.93 \\
\hline
\end{tabular}

For the present system, the $\mathrm{R}(3)$ absorption manifold was selected for measurement since it is one of the strongest yet most isolated absorption manifold within the $2 v_{3}$ band and the near-IR telecommunications spectrum. The selection of this manifold governs the design of the optical system, particularly the selected laser diodes, detectors, and wavelength sensitive fiber-based components. 


\section{Chapter 3 Experimental Design}

A modular TDLAS system was designed and constructed to permit quantitative testing of different hardware and signal-processing configurations (as detailed below) with the goal of developing a fiber-based system using all-optical sensor heads capable of measuring ambient methane in air at $\sim 1.7 \mathrm{ppm}_{\mathrm{v}}$. The TDLAS system interfaced with the all-optical sensor head via telecommunications-grade single-mode (SM) optical fibers networked to a common 'base-station' containing the lasers, detectors, and control system. Through optical multiplexing this arrangement would allow multiple sensor heads to be connected to a single base station.

\subsection{TDLAS System}

The TDLAS system was comprised of four sub-systems: the optical fiber network, the optical sensor head, the optical sub-systems, and the main control system. These are discussed separately under the headings that follow.

\subsubsection{Optical Fiber Network}

The optical network for this prototype TLDAS system was designed to accommodate easy reconfiguration during modification of the fiber-based sub-systems. Quantification of ambient methane over an array of fiber-optically coupled remote optical sensor heads can be achieved via multiplexing. Many methods of optical multiplexing exist in the optical telecommunications industry, and are generally used to increase the data bandwidth or provide redundancy within a network (Chan et al., 2000; Guan et al., 2007; Dakin, 1987; Baer et al., 1994; Tai et al., 1992; Rieker et al., 2007). Even though digital signals are not the transmitted content in the system being developed here, many multiplexing methods still apply, such as spatial (star), wavelength-division, and frequency-division multiplexing, as illustrated in Figure 3.1. 

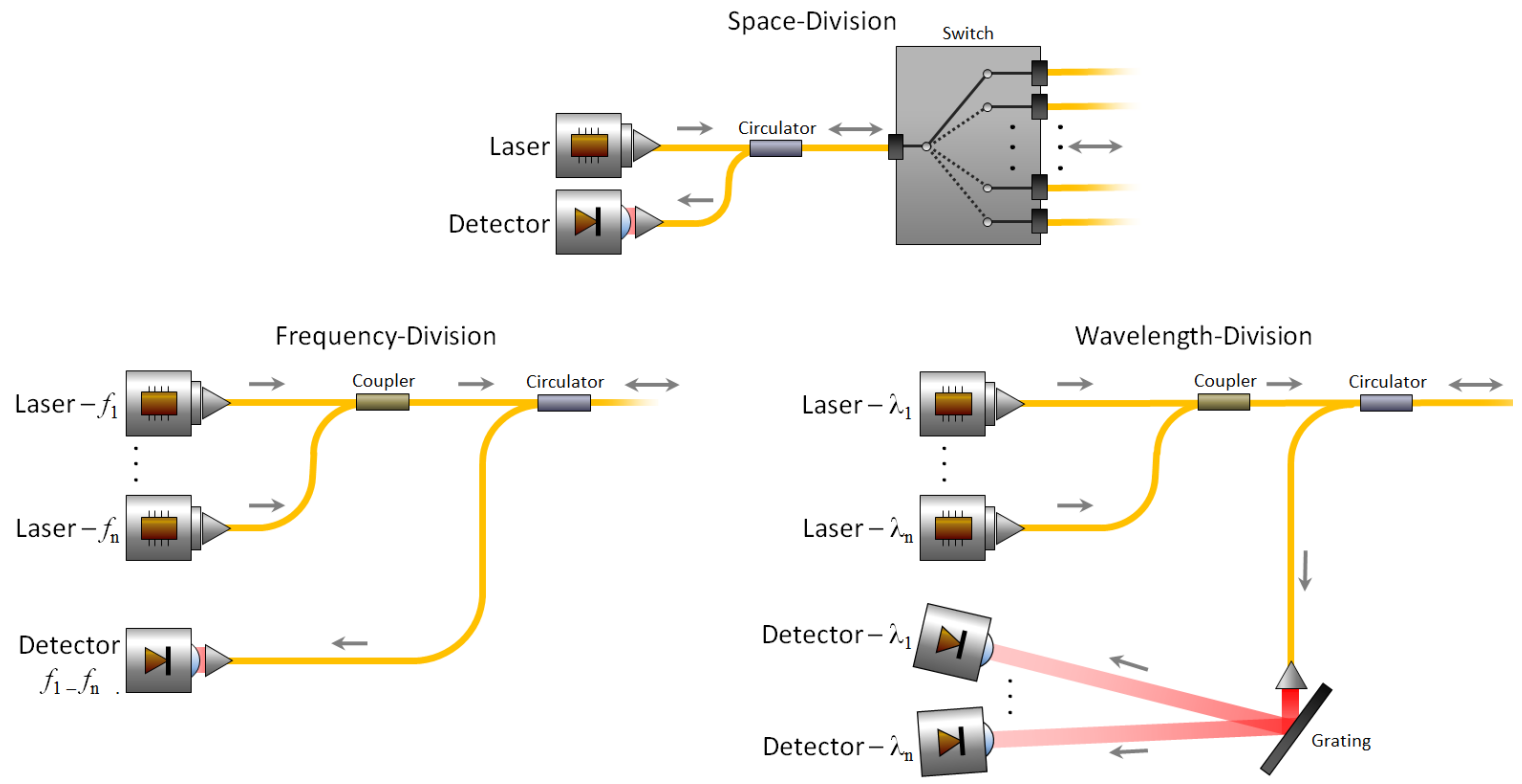

Figure 3.1: Fiber-optic multiplexing techniques; space-, frequency-, and wavelengthdivision multiplexing

The network configuration used in the present system is based on a space-division multiplexing scheme, with the potential for future expansion using combined frequencyand wavelength-division multiplexing. Spatial multiplexing is enabled by a telecommunications-grade bi-directional multi-channel optical relay, which interconnects the base TDLAS system to many remote optical sensor heads using dedicated SM fibers.

\subsubsection{Optical Methane Sensors}

The design of the optical sensor heads is primarily governed by the requirements of detecting ambient methane concentrations $\left(\sim 1.7 \mathrm{ppm}_{\mathrm{v}}\right)$ at ambient pressures and temperatures over an optical-fiber network. Long optical path-length sensors for spectroscopic measurement of gases are commercially available from companies such as Aerodyne Research Inc. and Boreal Laser Inc. However, these sensors are designed with the laser coupled directly to a detector, which leads to high individual sensor-head costs and complexities regarding intrinsic safety, limiting their applicability in a multi-sensor network. As a result, custom fiber-coupled optical sensor heads were designed and constructed to fulfil the particular requirements of the intended application. 
Short optical-path fiber-coupled interrogation cells generally employ two coaxial collimators on either side of the interrogation region for launching and coupling a laser beam to and from a fiber (Kinpui Chan et al., 1984; Chan et al., 1985; Tai et al., 1992; Edwards and Dakin, 1993; Stewart et al., 1998a; Whitenett et al., 2003; Culshaw, 2004). Though mechanically simplistic, this configuration is non-trivial to align, and results in unreliable coupling over long optical path-lengths, even within a controlled laboratory environment. In practice, an easier to align and more stable configuration utilized a retroreflector, a fiber circulator, and a single collimator for launching and receiving the laser beam into the interrogation region. This configuration also performed well at long optical-path lengths and was relatively straightforward to construct out of standard telecommunications-grade $9 / 125 \mu \mathrm{m}$ fiber-coupled optical components, as illustrated in Figure 3.2.

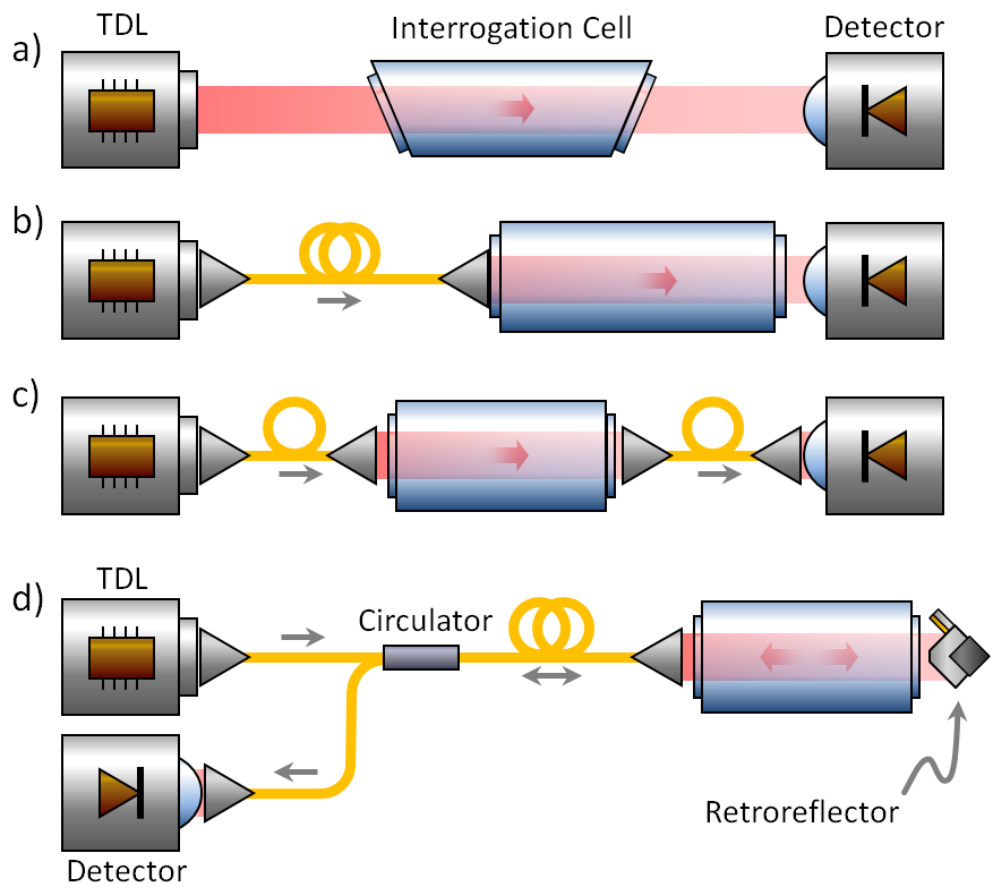

Figure 3.2: Generic optical routing configurations in and out of an interrogation region. a) straight through; b) fiber-in, detector out; c) dual fiber-in/-out; d) single fiber-in/-out

A retroreflector is an arrangement of three orthogonal planar reflectors which ensure the parallel alignment of incident and reflected beams, with an offset corresponding to the distance between the incident beam and the central axis of the retroreflector. If the incident beam can be directed onto the epicenter of the retroreflector 
(intersection of the three reflective surfaces) the reflected beam will travel co-axially with the incident beam vector. Alternatively, a retroreflector array could be used instead of a single retroreflector, but of the arrays tested (including retroreflective tapes and injectionmolded plastic arrays), none yielded return intensities as high as the single retroreflector.

An optical fiber connection to the sensor requires one or more collimators to transmit the laser beam to and from the interrogation region. A variety of commerciallyavailable aspheric lens collimators were tested, including pigtailed and directly fibercoupled, large and small apertures, variable and fixed focal lengths, and coated and uncoated lenses. Most of the collimators tested were designed for use around $1550 \mathrm{~nm}$, leading to excessive beam-divergence and low coupling efficiency within the 1650 $1665 \mathrm{~nm}$ spectral operating range of the present TDLAS system. Furthermore, many were constructed out of aluminum or stainless steel leading to a temperature dependence of the free-space beam characteristics. For these reasons a custom single-mode fiber coupled collimator was commissioned, which incorporated the desirable design attributes from commercially available units, but was optimized for use around $1650 \mathrm{~nm}$. The custom collimator (shown in Figure 3.3a, manufactured by OZ Optics) has a aspheric BK7 lens with a focal length of $18 \mathrm{~mm}$, housed within a thermally stable polymer casing to provide consistent beam characteristics over a wide temperature range. The collimating lens incorporates anti-reflective coatings to reduce fringing effects, improve transmission efficiency, and increase the beam coupling acceptance angle. The resultant near Gaussian laser beam has $4.0 \mathrm{~mm}$ diameter beam waist at approximately $10 \mathrm{~m}$, and an approximate divergence of 4 milliradians. 


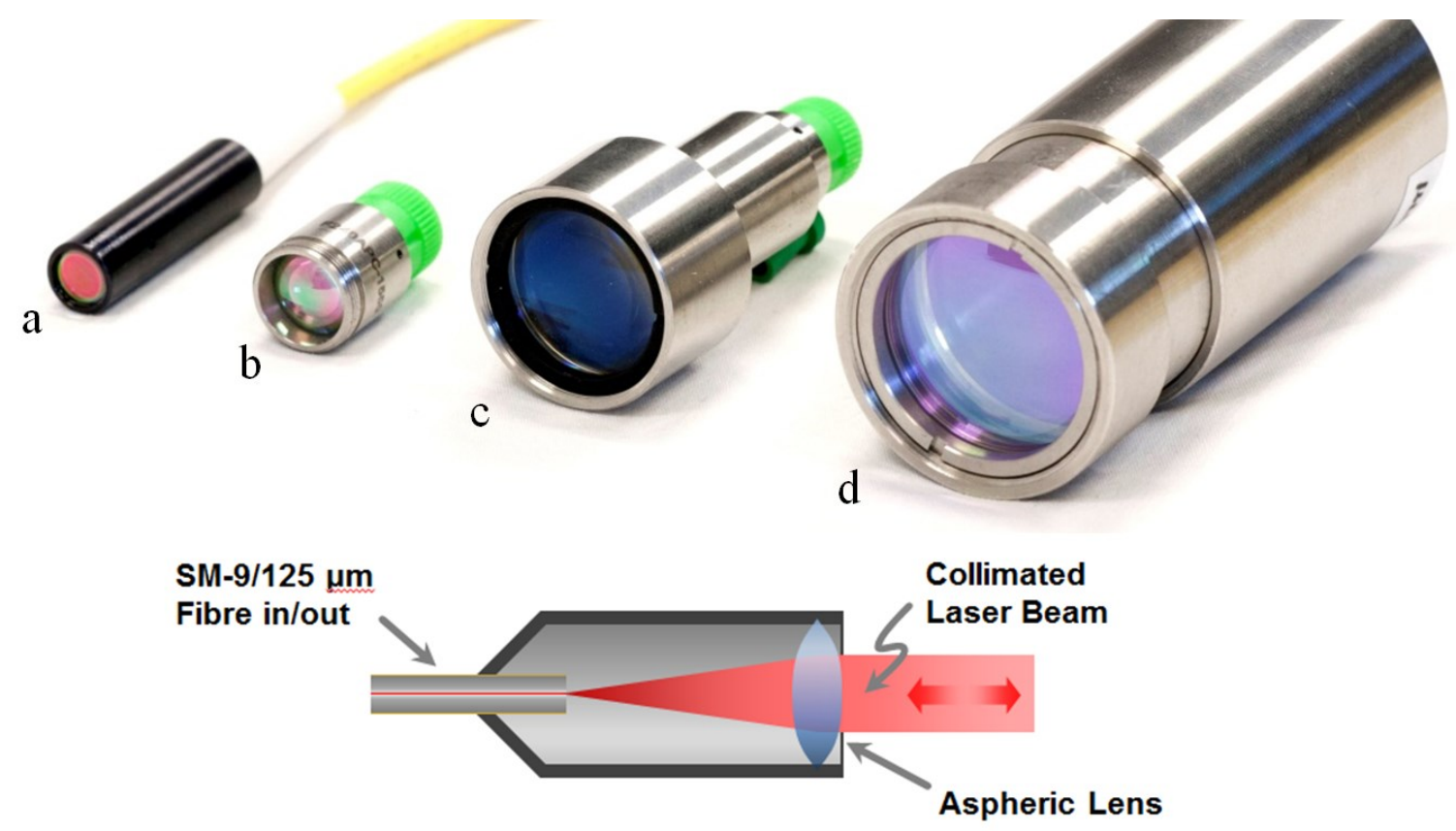

Figure 3.3: Lens collimators tested for use in the optical sensor. a) custom $\emptyset 4.0 \mathrm{~mm}$ beam, b) small $\emptyset 1.6 \mathrm{~mm}$ beam, c) doublet collimator $\emptyset 7.0 \mathrm{~mm}$ beam, micro laser systems FC20 $\emptyset 10.6 \mathrm{~mm}$ beam

\subsubsection{Component Performance}

To achieve a path-length sufficient for high-sensitivity continuous-wave laser absorption spectroscopy, a folded optical path was required. However, misalignment sensitivity grows as the folded optical path-length is increased. Misalignment can be introduced by mechanical vibration, manufacturing imperfections, and temperature fluctuations, and can be further exacerbated by beam-steering (i.e. refraction of the beam due to index of refraction gradients within the sample cell). Beam scattering and wavelength-dependant issues were minimized by using reflective surfaces rather than refractive surfaces wherever possible (Werle, 1998; Hastie and Miller, 1985; Cvijin et al., 1992). Surfacereflecting 'hollow' reflectors were used (manufactured by PLX Inc.) to route the beam within the interrogation cell. Losses due to scattering and mechanical tolerances were minimized using a high quality surface roughness of $\lambda_{(633 \mathrm{~nm})} / 10$, a low beam deviation of less than 2.0 arcseconds, and a monolithic arrangement of the reflective surfaces.

Performance characteristics were obtained for six optical configurations to assess the influence of specific components and optical path-length on ease of alignment and 
optical coupling efficiency. The configurations tested have folded optical path-lengths between 0.5 and $6 \mathrm{~m}$, use up to four optical elements, and introduce up to 15 reflections and 8 passes through the interrogation region. Figure 3.4 illustrates the optical component configurations created on an optical bench using combinations of a collimator, roof mirror, retroreflector, and free-space detector.

A)

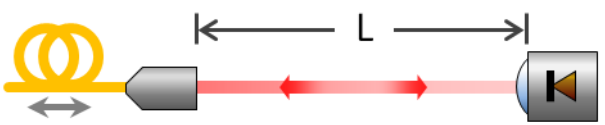

B)

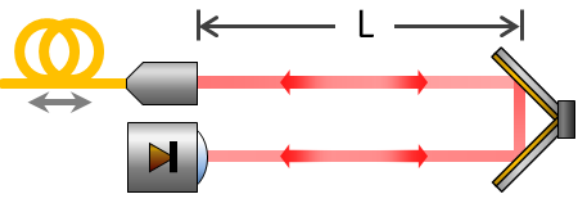

C)

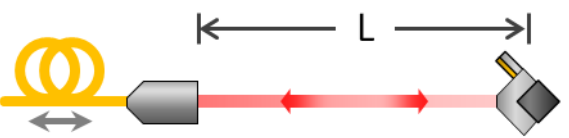

D)

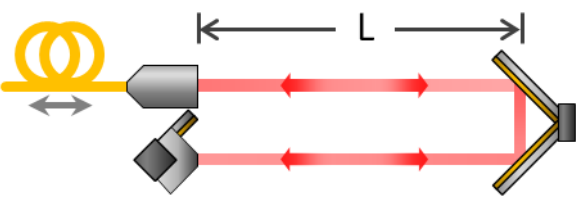

Collimator

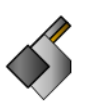

Retroreflector
E)
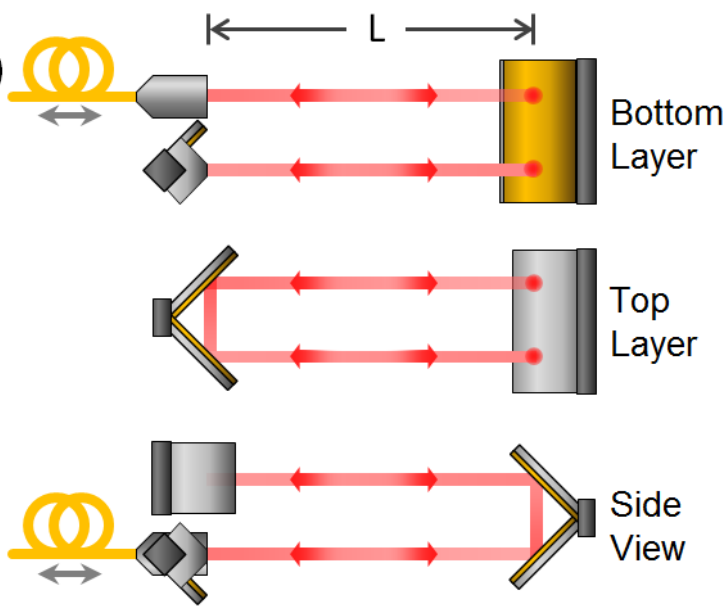

Figure 3.4: Component configurations used to test the influence of optical path length and components on return intensity and absorption signal strength

Configurations A, B, C, D, and E were used to assess the influence of optical component configurations and path-length on coupling efficiency and measurement stability. The physical distance between the optics on each side of the test configuration interrogation regions was limited to $0.75 \mathrm{~m}$ by the physical dimensions of the optical breadboard and test section, which in turn limited the achievable optical path-lengths to those specified in Table 3.1. The performance of these configurations was assessed by:

- Return intensity at a free-space or fiber-coupled detector depending on the configuration, 
- Signal strength of absorption and second-harmonic signals generated at $\sim 1.7 \mathrm{ppm}_{\mathrm{V}}$ and $100 \mathrm{ppm}_{\mathrm{V}}$ of methane in air at standard pressures and temperatures, and

- Measurement scatter of a second-harmonic spectroscopic feature, with an averaging time of $1 \mathrm{~s}$.

Table 3.1: Optical configuration technical data

\begin{tabular}{|c|c|c|c|c|c|c|c|c|c|c|c|}
\hline \multirow[b]{2}{*}{ Name } & \multirow[b]{2}{*}{ Config. } & \multicolumn{2}{|c|}{ Detection } & \multicolumn{2}{|c|}{ Collimator } & \multicolumn{2}{|c|}{$\begin{array}{c}\text { Optical } \\
\text { Element(s) }\end{array}$} & \multicolumn{2}{|c|}{ Path-Length [m] } & \multirow[b]{2}{*}{ Passes } & \multirow{2}{*}{$\begin{array}{c}\# \text { of } \\
\text { Reflections }\end{array}$} \\
\hline & & FC & FS & Lens & Mirror & Retro & Roof & Physical & Optical & & \\
\hline$\overline{\mathrm{A}_{\mathrm{M} 0.25}}$ & $\bar{A}$ & & $\checkmark$ & & $\checkmark$ & & & 0.25 & 0.25 & 1 & 0 \\
\hline $\mathrm{A}_{\mathrm{L} 0.25}$ & A & & $\checkmark$ & $\checkmark$ & & & & 0.25 & 0.25 & 1 & 0 \\
\hline $\mathrm{B}_{\mathrm{M} 0.5}$ & B & & $\checkmark$ & & $\checkmark$ & & 1 & 0.25 & 0.5 & 2 & 2 \\
\hline $\mathrm{B}_{\mathrm{M} 1.0}$ & B & & $\checkmark$ & & $\checkmark$ & & 1 & 0.50 & 1.0 & 2 & 2 \\
\hline $\mathrm{B}_{\mathrm{L} 0.5}$ & B & & $\checkmark$ & $\checkmark$ & & & 1 & 0.25 & 0.5 & 2 & 2 \\
\hline $\mathrm{B}_{\mathrm{L} 1.0}$ & B & & $\checkmark$ & $\checkmark$ & & & 1 & 0.50 & 1.0 & 2 & 2 \\
\hline $\mathrm{C}_{\mathrm{M} 0.5}$ & $\mathrm{C}$ & $\checkmark$ & & & $\checkmark$ & 1 & & 0.25 & 0.5 & 2 & 1 \\
\hline $\mathrm{C}_{\mathrm{M} 1.0}$ & $\mathrm{C}$ & $\checkmark$ & & & $\checkmark$ & 1 & & 0.50 & 1.0 & 2 & 1 \\
\hline $\mathrm{C}_{\mathrm{L} 0.5}$ & $\mathrm{C}$ & $\checkmark$ & & $\checkmark$ & & 1 & & 0.25 & 1.0 & 2 & 1 \\
\hline $\mathrm{C}_{\mathrm{L} 1.0}$ & $\mathrm{C}$ & $\checkmark$ & & $\checkmark$ & & 1 & & 0.50 & 1.0 & 2 & 1 \\
\hline $\mathrm{D}_{\mathrm{M} 1.0}$ & $\mathrm{D}$ & $\checkmark$ & & & $\checkmark$ & 1 & 1 & 0.25 & 1.0 & 4 & 7 \\
\hline $\mathrm{D}_{\mathrm{M} 2.0}$ & D & $\checkmark$ & & & $\checkmark$ & 1 & 1 & 0.50 & 2.0 & 4 & 7 \\
\hline $\mathrm{D}_{\mathrm{M} 3.0}$ & $\mathrm{D}$ & $\checkmark$ & & & $\checkmark$ & 1 & 1 & 0.75 & 3.0 & 4 & 7 \\
\hline $\mathrm{D}_{\mathrm{L} 1.0}$ & D & $\checkmark$ & & $\checkmark$ & & 1 & 1 & 0.25 & 1.0 & 4 & 7 \\
\hline $\mathrm{D}_{\mathrm{L} 2.0}$ & $\mathrm{D}$ & $\checkmark$ & & $\checkmark$ & & 1 & 1 & 0.50 & 2.0 & 4 & 7 \\
\hline $\mathrm{D}_{\mathrm{L} 3.0}$ & D & $\checkmark$ & & $\checkmark$ & & 1 & 1 & 0.75 & 3.0 & 4 & 7 \\
\hline $\mathrm{E}_{\mathrm{L} 2.0}$ & E & $\checkmark$ & & $\checkmark$ & & 1 & 2 & 0.25 & 2.0 & 8 & 15 \\
\hline $\mathrm{E}_{\mathrm{L} 4.0}$ & E & $\checkmark$ & & $\checkmark$ & & 1 & 2 & 0.50 & 4.0 & 8 & 15 \\
\hline $\mathrm{E}_{\mathrm{L} 6.0}$ & E & $\checkmark$ & & $\checkmark$ & & 1 & 2 & 0.75 & 6.0 & 8 & 15 \\
\hline
\end{tabular}

Figure 3.5 shows the intensity and $2 f$ and absorption signal-to-noise ratio (SNR) results as a function of varying optical path length for both the WMS and SI measurement techniques for the tested optical configurations. SNR is defined here as, $\mathrm{SNR}=\bar{x} / s_{x}$, where $\bar{x}$ and $s_{x}$ are the mean and $1 \sigma$ standard deviation of the experimentally measured absorption or $2 f$ values over an averaging time of one-second. The SI results were obtained at a sweep frequency of $300 \mathrm{~Hz}$, while the WMS results were obtained using a sweep frequency of $100 \mathrm{~Hz}$ and a modulation frequency of $20 \mathrm{kHz}$. Data were acquired at the $2 v_{3} \mathrm{R}(3)$ methane absorption manifold, ambient pressures and temperatures, and with the various optical sensor heads coupled to the base station with a 6-m 9/125 $\mu \mathrm{m}$ fiber optic cable. 


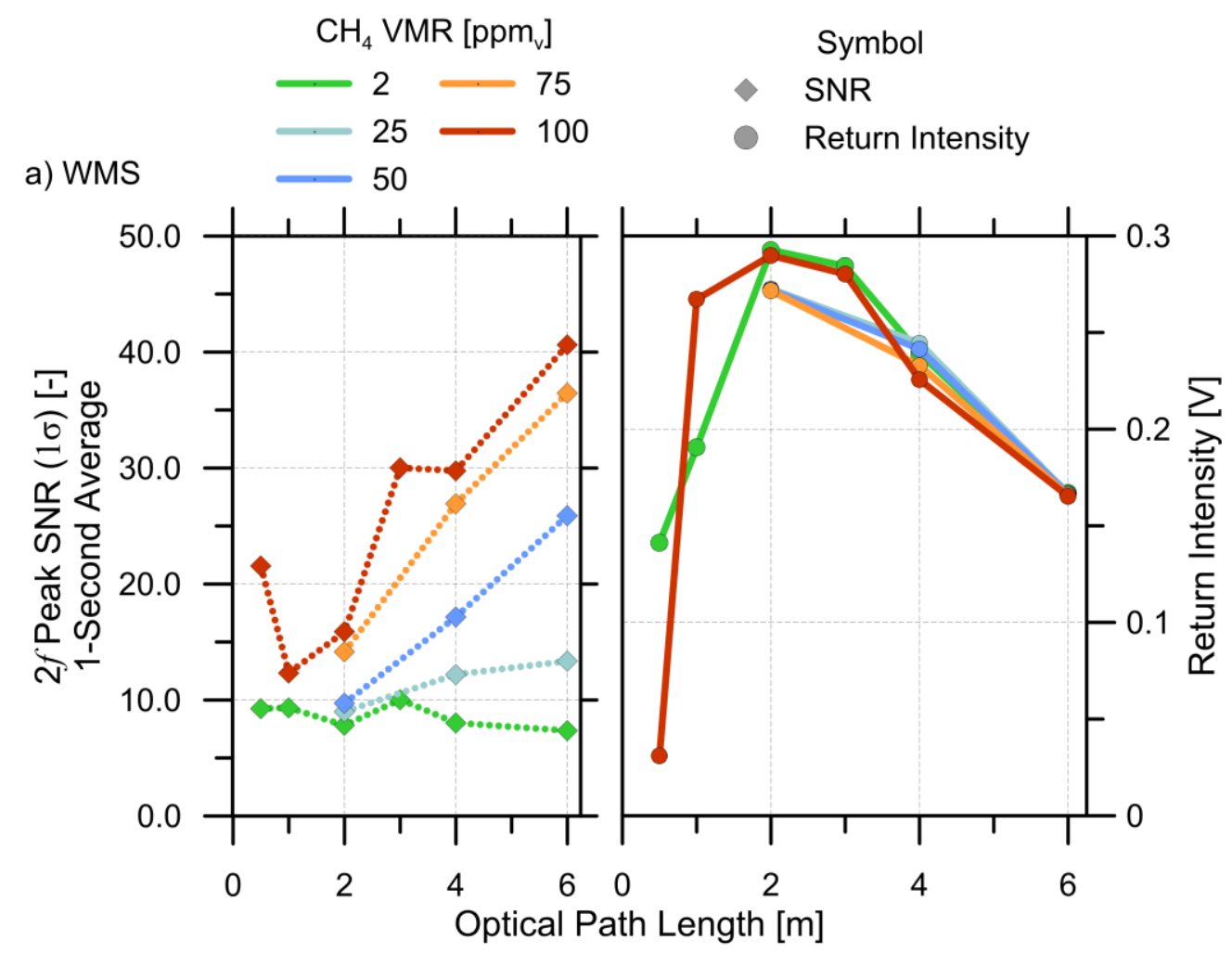

b) $\mathrm{SI}$

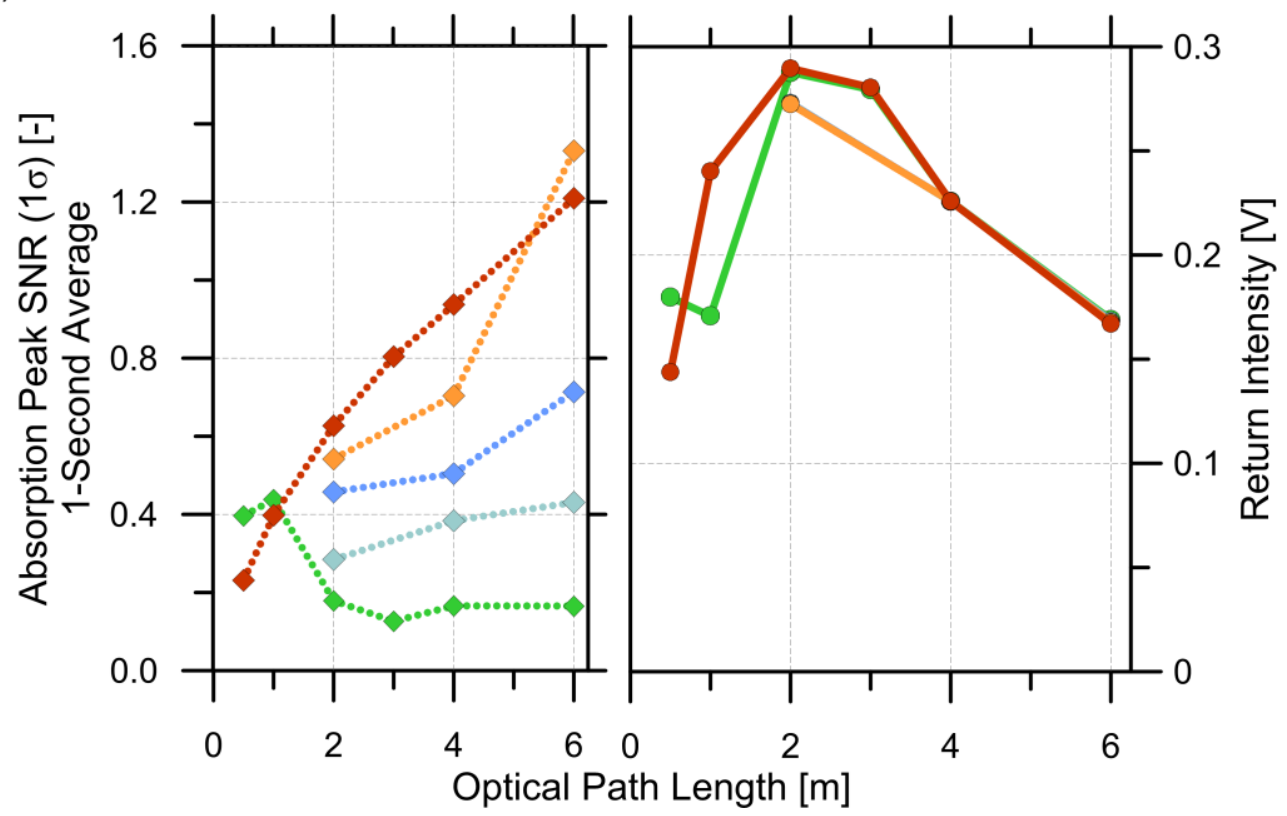

Figure 3.5: Effects of methane volume mixing ratio and optical path length on SI (absorption) and WMS (second-harmonic, $2 f$ ) feature SNR, and return intensity. The SI results were obtained at a sweep frequency of $300 \mathrm{~Hz}$, while the WMS results were obtained using a sweep frequency of $100 \mathrm{~Hz}$ and a modulation frequency of $20 \mathrm{kHz}$. Data was acquired at the $2 v_{3} R(3)$ methane absorption manifold, ambient pressures and temperatures, and with the various optical sensor heads coupled to the base station with a 6-m 9/125 $\mu \mathrm{m}$ fiber optic cable. 
The signal-to-noise ratio (SNR) of the measured absorption feature peak is at least 4 times smaller than the SNR of the $2 f$ peak at equivalent optical path lengths and absorbing gas concentrations. Generally, for volume mixing ratios above ambient (25 $\mathrm{ppm}_{\mathrm{v}}$ and above in the figure) the SNR increases with optical path length for both methods. Interestingly, at concentrations of $2 \mathrm{ppm}_{\mathrm{v}}$, the SNR of the absorption and $2 f$ feature maxima remains approximately constant (or even decreases slightly) with increasing optical path-length. For all cases in the available data, the return intensity reached a maximum at a path length of $2 \mathrm{~m}$, and decreased thereafter as pathlength increased. This is likely attributable to beam divergence. In general, the number of reflections had negligible effect on SNR compared to the effects of increased optical path-length, suggesting that an optimal optical sensor head design would preferentially maximize optical path-length.

\subsubsection{Multipass Open-Path Sensor Head Design}

Stable, long optical path length cells are commercially available, but are generally expensive, of the closed-cell flow-through variety, and have no option for coupling the laser beam back in to a fiber optic cable. After developing a series of multipass sensor head prototypes, a unique configuration of reflective surfaces was developed which permitted bi-directional fiber-coupling to an open-path sensor head. The multipass configuration consists of a pair of roof mirrors, a retroreflector, and a lens-type collimator similar to what is illustrated in Figure 3.4 (E). The roof mirrors consist of orthogonal reflective surfaces, and are affixed precisely at each end of an interrogation region, one providing a horizontal beam shift, and the other providing a vertical beam shift. The beam makes a total of 8 passes through the interrogation region, and is reflected a total of fifteen times off the surfaces of the roof mirrors and retroreflector prior to being directed back into the same collimator used to launch the beam from the fiber, resulting in a 5.79 meter optical path-length within a 75 centimeter long ( $\sim 9$ litre volume). The reflectors are arranged to minimize the beam degrees of freedom, governed by the physical location, orientation, and stability of the reflective components. The reflective surfaces are mounted to kinematic mounts to enable the correction of beam misalignment 
due to manufacturing or assembly limitations. The mounts provide precise positioning control in the axes found to be most sensitive to misalignment.

Within the optical sensor head, the arrangement of reflective surfaces relies more on the manufacturing tolerances of the reflective optics than assembly tolerances and only requires two independent optical mounts for final alignment. Figure 3.6 shows a photo of the final prototype; the beam path and optical component configuration is illustrated in Figure 3.7.

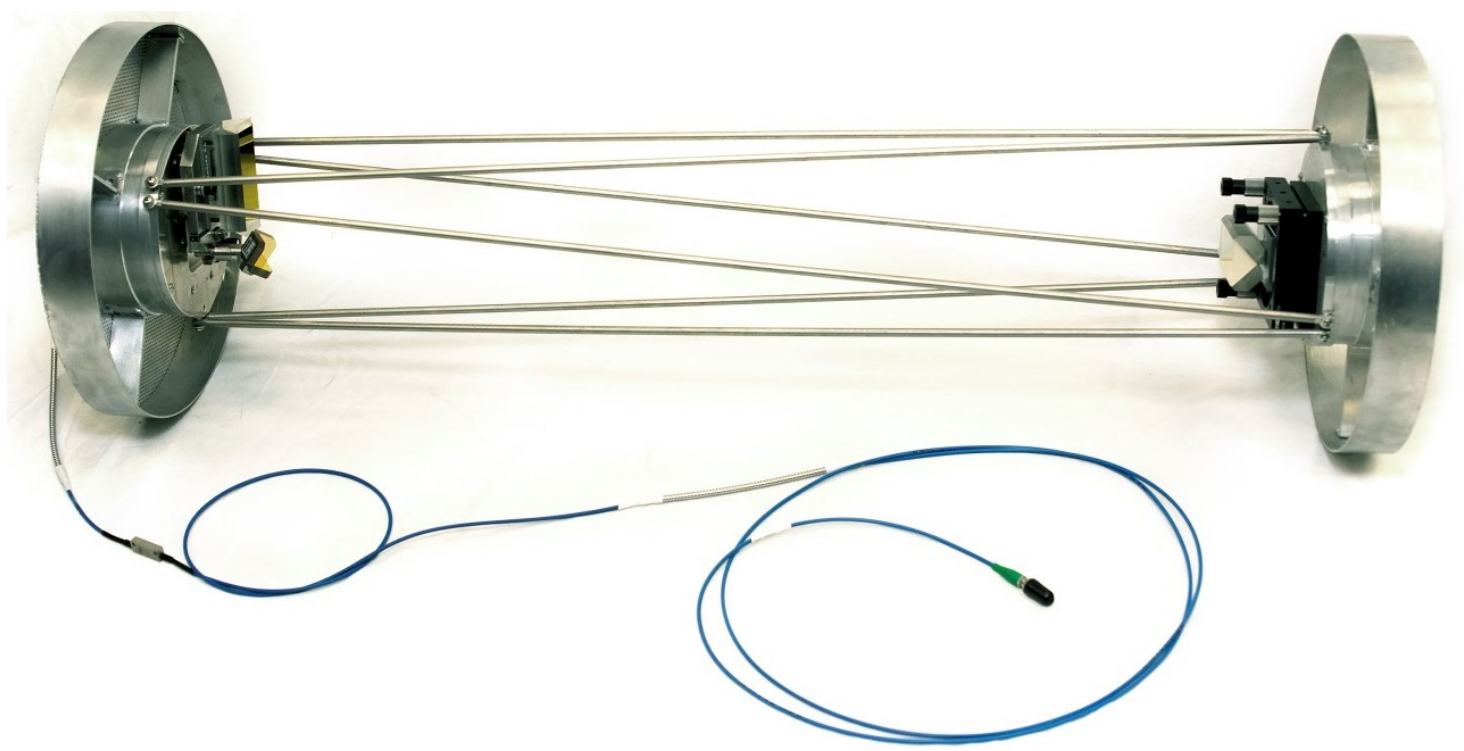

Figure 3.6: Optical fiber-coupled 5.79 meter open-path multipass optical sensor head prototype; demonstrated here with the removable test chamber support structures on the ends

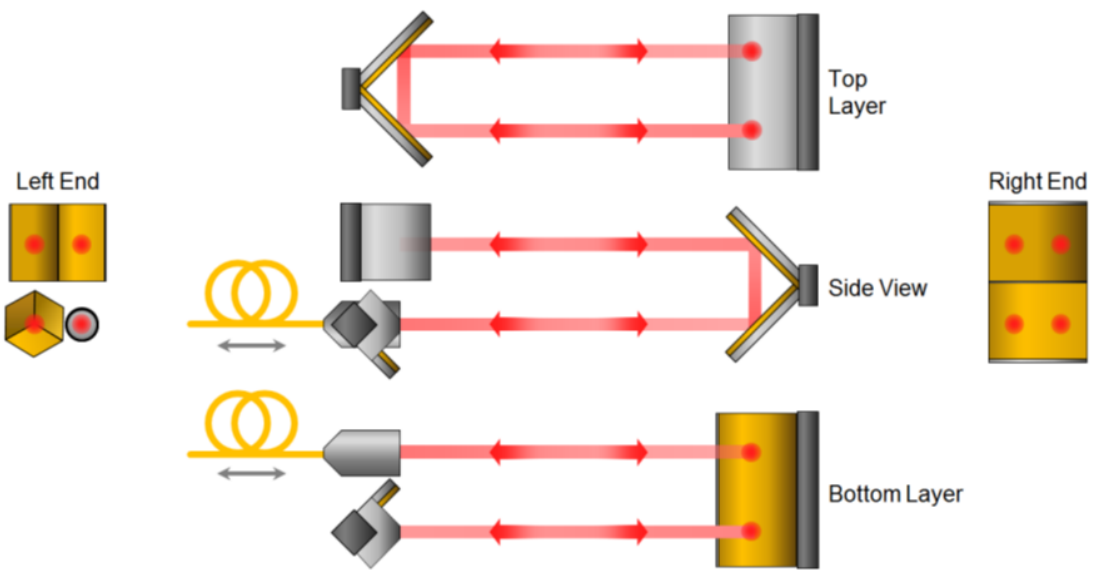

Figure 3.7: Illustration of the laser beam path though the fiber coupled optical sensor head 
The configuration of the optical components within the optical sensor provided more mechanical and long-term stability than the same optical components arranged using optical mounts fastened to an optical breadboard. Even though the optical sensor head was moved between laboratories and was repeatedly cycled in and out of the test chamber, the final prototype did not require realignment of the reflective surfaces for more than three years. Interrogation cell designs comprised of a collimating lens and/or retroreflector have been employed by others (Chambers et al., 2004; Edwards and Dakin, 1993; Li, 2006; Chernin, 2001; Durry and Pouchet, 2002; Altmann et al., 1981; Silver, 2005; Hu et al., 1993; Linnartz, 2004), but to the author's knowledge, the present configuration of optical components has not been demonstrated previously.

To achieve stable reflector geometry while minimizing weight and simplifying construction, the aluminum mirror-mount end-plates were supported using a triangular truss structure. To ensure alignment over the operational temperature range, the support rods were made of an Iron-Nickel alloy (Invar ${ }^{\mathrm{TM}}$ ) which possesses a low coefficient of thermal expansion, 10-15 times less than steel or aluminum.

\subsubsection{Optical Systems}

\subsubsection{Optical System Diagram}

The test TDLAS system employed fiber optic routing for robustness and compactness, and makes use of fiber-coupled tunable diode lasers, detectors, reference gas cell, and resonator. Figure 3.8 shows a schematic of the complete system, consisting of the base TDLAS system and fiber-coupled optical sensor head. High-bandwidth low-loss telecommunications grade SM fibers were used throughout the optical sub-system. SM fibers were selected over polarization maintaining or multimode fibers due to their lower cost and higher bandwidth. Wherever the fiber cables were at risk for continued bending or wear, jacketed fibers reinforced with a layer of Aramid ${ }^{\mathrm{TM}}$ yarn were used. In areas where flexibility was more critical than durability, $900 \mu \mathrm{m}$ loose-tube fibers were used, which were easier to handle at the expense of being more fragile. The physical fiber connections between modular sections were made with high-polish face-centered angled physical connections (FC/APC) to minimize back-reflections and unwanted optical 
interference. FC/APC connectors are not as widely used in the telecommunications industry because of their cost and the care needed to prevent damage while coupling, but provide high repeatability and less back-reflection over more common plastic snap connectors. Furthermore, by implementing FC/APC fiber connections, optical interference originating at the interface can be drastically reduced (Jin et al., 1997).

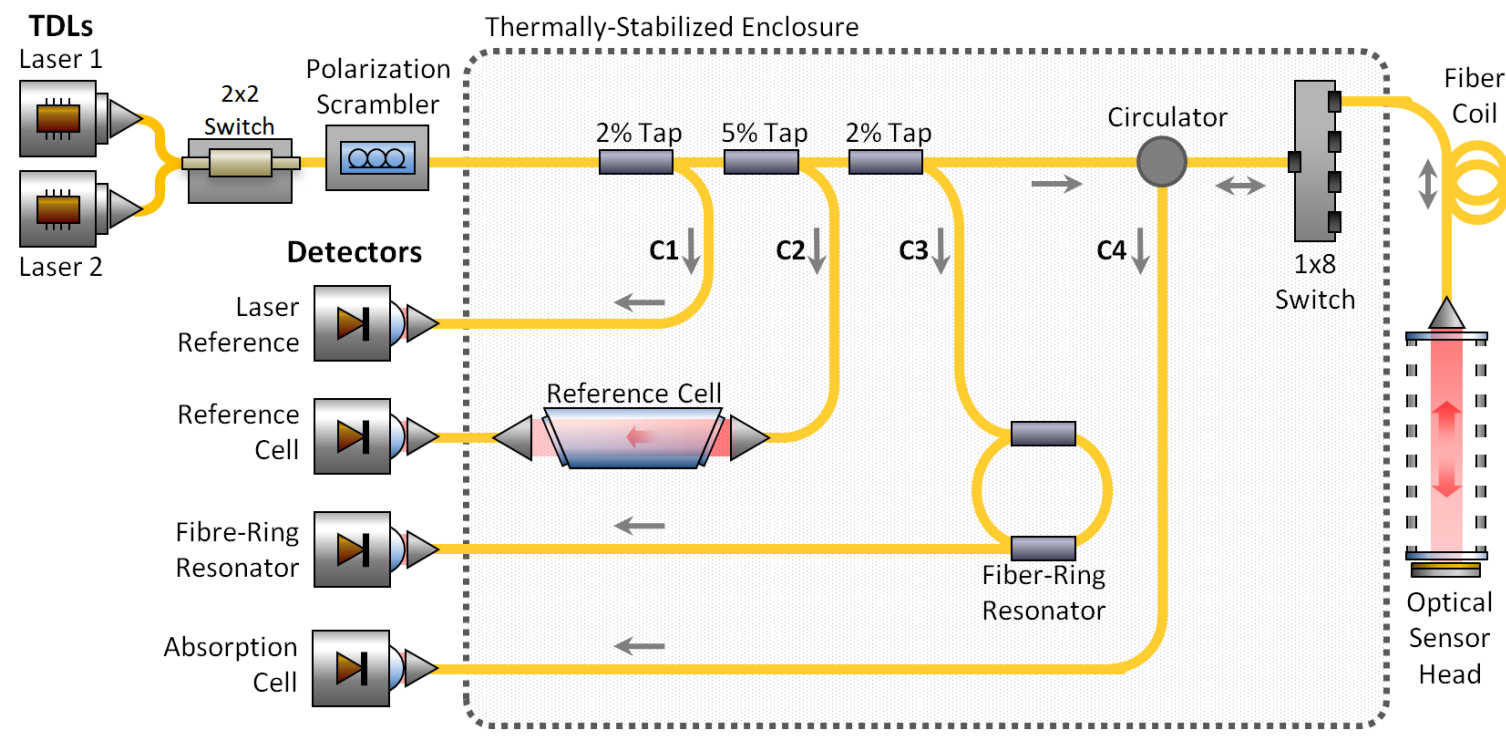

Figure 3.8: Optical sub-system schematic; (A) 2-by-2 switch and controller, (B) AVOA, (C) taps, laser reference $(\mathrm{C1})$, reference cell $(\mathrm{C} 2)$, FRR (C3), and multiplexing (C4) routes

The light from the tunable diode lasers was routed into a 2-by-2 switch (A), which routed one of the two inputs into the system, and the other into an optical terminator. The 2-by-2 switch is an Agiltron Nanospeed ${ }^{\mathrm{TM}}$ low-loss microelectromechanical system (MEMS) switch; which provided fast, repeatable, long-life, remote selection of one of the two laser diodes. Immediately following the 2-by-2 switch and a General Photonics polarization scrambler the optical signal was split into four separate routes via $2 \%$ couplers to permit simultaneous intensity or wavelength measurement via four parallel detectors, discussed further in Section 3.1.3.4. A coupler is a passive device which splits light from a single input fiber into two or more output fibers, and is ideally suited for minimizing back-reflections and maintaining the integrity of the input optical signal. Routes C1 - C3 employed manual optical attenuators to limit the optical power passing though the fiber to prevent saturation and facilitate equal amplification across all detectors. 
Routes $\mathrm{C} 1$ to $\mathrm{C} 3$ are the laser reference, reference cell, and fiber-ring resonator routes, which were used to provide laser monitoring, wavelength references, signal synchronization, and system calibration, as discussed further in Section 4.4. The multiplexing route $(\mathrm{C} 4)$ was comprised of a 3-port circulator, an 8-channel multiplexer, the remote optical sensor head(s), and an interchangeable coil of optical fiber. The 8channel optical relay (or spatial multiplexer) was custom-built by Agiltron, and included similar Nanospeed ${ }^{\mathrm{TM}}$ MEMS optics, a control system, and performance specifications similar to the above-mentioned 2-by-2 switch. A custom-built circulator separated the incoming and outgoing optical signals allowing bi-directional transmission to the optical sensor head(s) via the optical relay. Multiple fiber delay-coil lengths between 1 and 1000 meters could be manually interchanged immediately after the optical switch to test the effects of fiber-length (between the base system and optical sensor head) on the system and measurement results.

The fiber-optic components (i.e. taps, couplers, circulator, optical attenuators, and fiber-ring resonator (FRR), reference cell, and optical relay) were housed within a thermally stabilized enclosure. These components do not share a similar sensitivity to temperature, but have been housed within the same enclosure to permit permanent splicing between the components and to minimize the overall assembly footprint. The temperature control system was a CP-200 TEC from TE-Technology with a heating and cooling capacity of $200 \mathrm{~W}$. The enclosure was a standard 9" rack-mount drawer internally lined with layers of $1.5 "(37.5 \mathrm{~mm})$ thick closed-cell foam and Aerogel ${ }^{\mathrm{TM}}$ insulation. To ensure efficient heat transfer between the TEC and the optical components, the components were mounted onto a polished 0.5 " thick aluminum plate (12" by 10.5 ") with an approximate mass of $2.8 \mathrm{~kg}$. The aluminum plate was screwed to the Peltier element using thermally-conductive paste between the mating surfaces, and the Peltier element was bonded to a large area heat sink and fan, as illustrated in Figure 3.9 . 

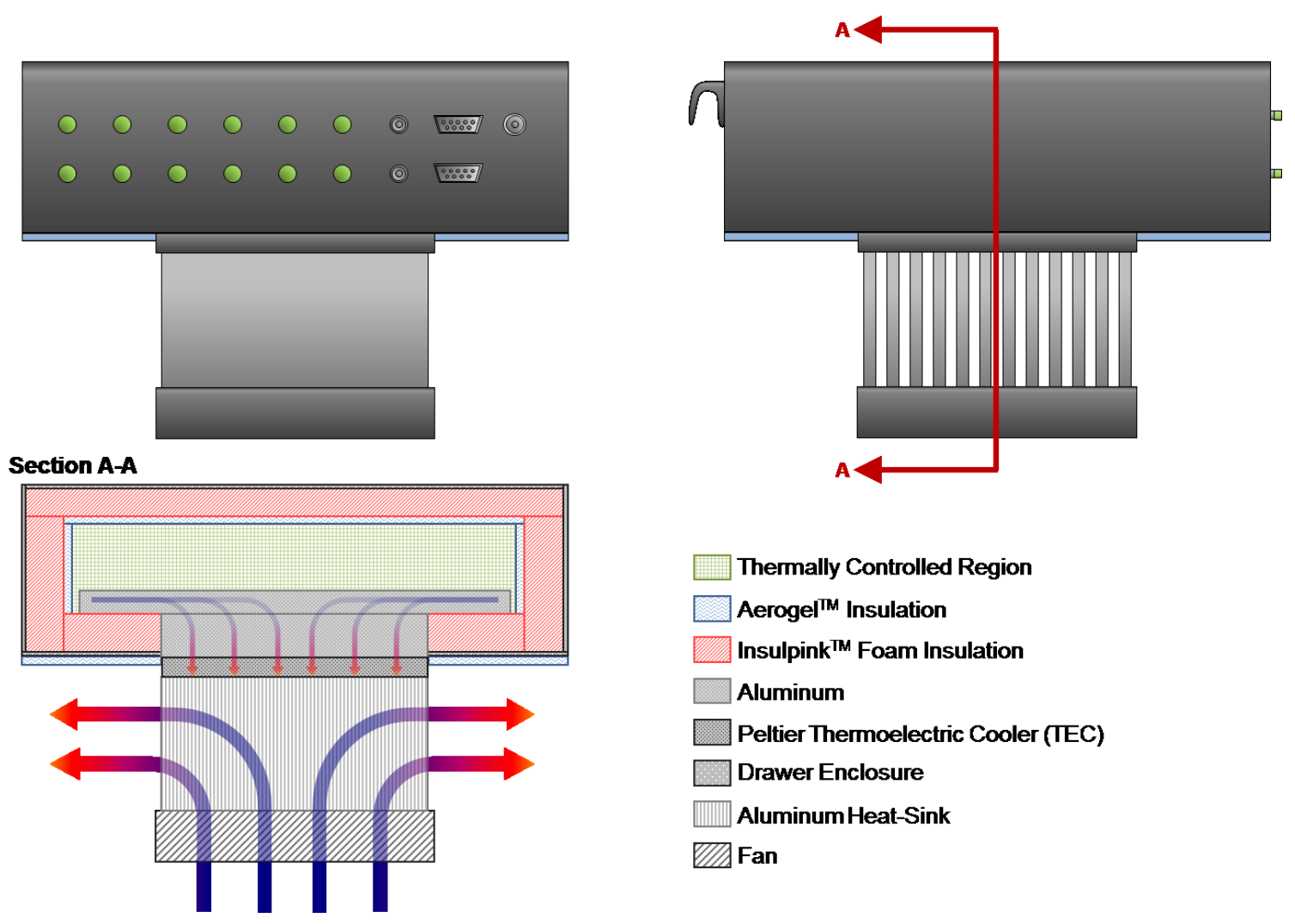

Figure 3.9: Thermally stabilized enclosure exterior and cross-section (A-A)

Thermocouples within the enclosure provided feedback to the TEC controller for thermal stabilization of the aluminum plate and interior air-space. At laboratory temperatures near $23^{\circ} \mathrm{C}$, the temperature within the enclosure required approximately $7 \mathrm{~W}$ to sustain a stable internal temperature of $20.10^{\circ} \mathrm{C}$. The temperature within the enclosure was held a few degrees lower than ambient temperature to limit power consumption, ensure constant cooling, and maintain a temperature within the intended operating ranges of the optical components

The potential utility of a polarization scrambler (General Photonics PCD-104, implementing a multiple stage birefringent modulator via four piezoelectric transducers) for mitigating the influence of polarization variation on remote methane volume mixing ratio measurements was tested. This polarization scrambler was modular and could be swapped in and out of the system between the absorption laser (Laser 2) and thermal enclosure. The function and performance of the polarization scrambler in reducing intensity drift is discussed further in Chapter 5. 


\subsubsection{Photodetectors}

Optical intensities were measured using four fiber-coupled indium gallium arsenide (InGaAs) semiconducting photodiodes operating in photoconductive mode (OptoSci LNP2-A). To limit electronic noise on the output signals, the photodiodes were electromagnetically shielded and powered by a dedicated low-noise line-isolated switching power supply. The specifications of the laser diodes are listed in Table 3.2.

Table 3.2: TDLAS System Detector Specifications

\begin{tabular}{l|c|c}
\hline \multicolumn{1}{c|}{ Parameter at $\mathbf{1 6 5 0} \mathbf{n m}$} & Units & Value \\
\hline Detector Type & - & InGaAs \\
Package & - & FC/PC Bulkhead \\
Detector Diameter & $\mathrm{mm}$ & 0.5 \\
Noise Equivalent Power (NEP) & $\mathrm{W} / \sqrt{\mathrm{Hz}}$ & $2.0 \times 10^{-14}$ \\
Responsivity & $\mathrm{A} / \mathrm{W}$ & $0.8 \pm 0.1$ \\
CW Saturation Power & $\mathrm{mW}$ & 2.0 \\
Dark Current (Maximum) & $\mathrm{mA}$ & 0.15 \\
Bandwidth (for all Gain levels) & $\mathrm{Hz}$ & 0.01 to $5 \times 10^{5}$ \\
Transimpedance Gain & $\mathrm{V} / \mathrm{A}$ & $10^{3}$ to $10^{5}$ \\
\hline
\end{tabular}

The small active area on the photodiodes permitted a high frequency response, but limited the incident optical power to approximately $2.0 \mathrm{~mW}$. Each photodiode had dedicated analog amplification along with $\mathrm{AC}$ and $\mathrm{DC}$ coupling modes to permit basic analog signal processing, at the disadvantage of introducing bandwidth limitations and increasing electrical noise. Since return power was sufficiently high, analog amplification was not necessary. Instead, the detectors were paired with a sensitive analog to digital converter which provided a flexible 16-bit resolution over the working range of the photodetectors.

Over the operating spectral range of the TDLAS system $(1650-1665 \mathrm{~nm})$ the photodiodes had a steep responsivity (output current per input optical power $[\mathrm{A} / \mathrm{W}]$ ) which was further complicated by strong temperature dependence, as shown in Figure 3.10 . 


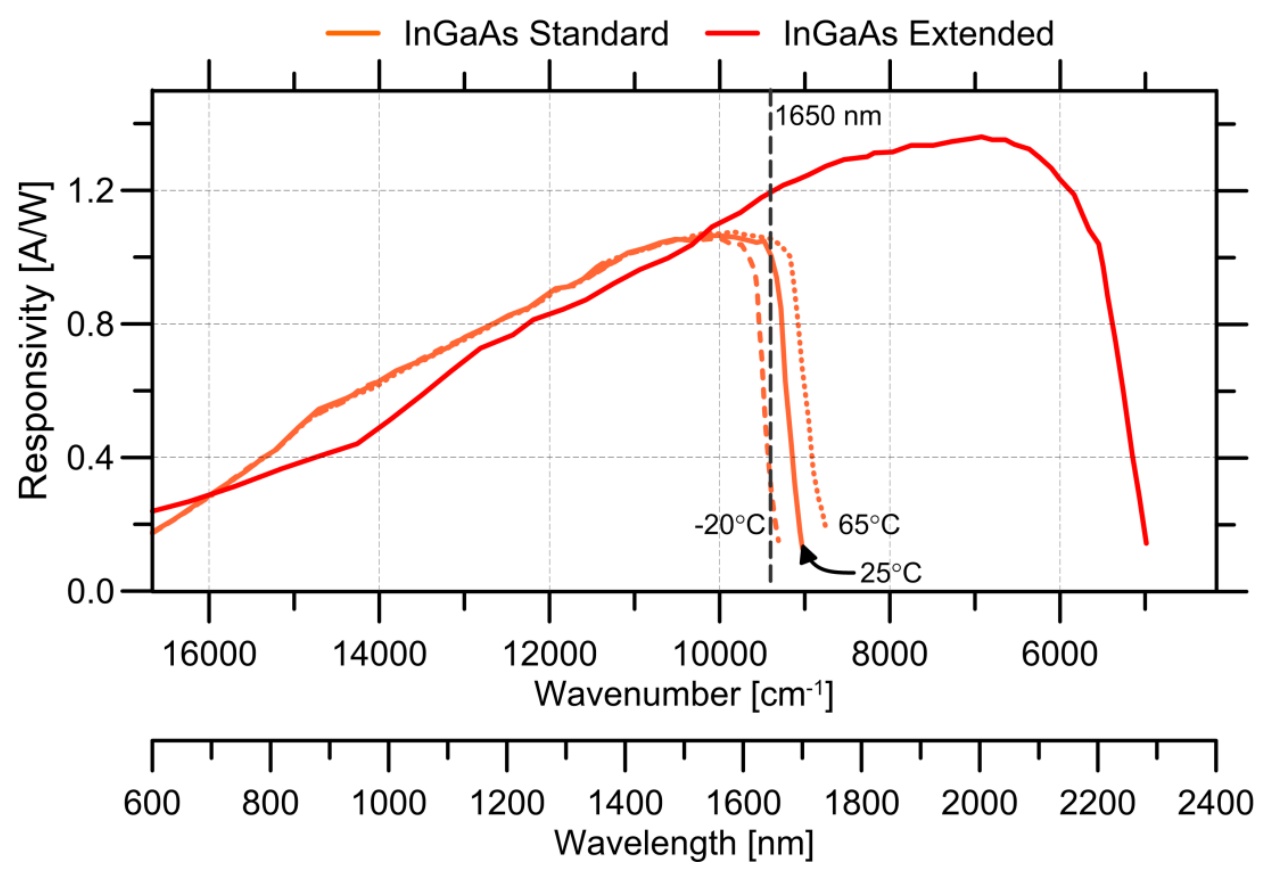

Figure 3.10: InGaAs and extended InGaAs detector responsivity curves. Adapted from (Laser Components, 2015a; Laser Components, 2015b)

The photodetectors were not thermally stabilized in this system, so simulations were conducted to estimate the influence of photodetector responsivity on measurement performance for the SI and WMS techniques at laser intensities similar to what was observed experimentally. The simulations were based on experimental measurements using the fiber-coupled $5.79 \mathrm{~m}$ optical sensor head containing 0 and $25 \mathrm{ppm}_{\mathrm{v}}$ methane in air at $23^{\circ} \mathrm{C}$ and $101.325 \mathrm{kPa}$. Measurement precision $(2 \sigma)$ was estimated using the $95 \%$ prediction intervals from a linear best-fit of simulated sensor head return intensity data averaged for one-second, calculated after white noise (derived from the scatter on return intensity from the optical head while the test section was purged with nitrogen at ambient pressure and temperature) was applied to simulated intensity signals before absorption. Figure 3.11(a) illustrates that for an increase in photodetector responsivity from 0.6 to $0.69 \mathrm{~A} / \mathrm{W}$ (corresponding to an approximate increase in photodiode temperature from $22^{\circ} \mathrm{C}$ to $32^{\circ} \mathrm{C}$ at $1653 \mathrm{~nm}$ ), for the SI technique methane volume mixing ratio measurement precision $(2 \sigma)$ decreases from about 5.5 to $5.0 \mathrm{ppm}_{\mathrm{v}}$ at a methane VMR of $2 \mathrm{ppm}_{\mathrm{v}}$. In contrast, for the WMS technique, photodetector responsivity had little influence on the measurement precision $(2 \sigma)$ of $0.56 \mathrm{ppm}_{\mathrm{v}}$ at $2 \mathrm{ppm}_{\mathrm{v}}$ as illustrated in Figure 3.11(b). 


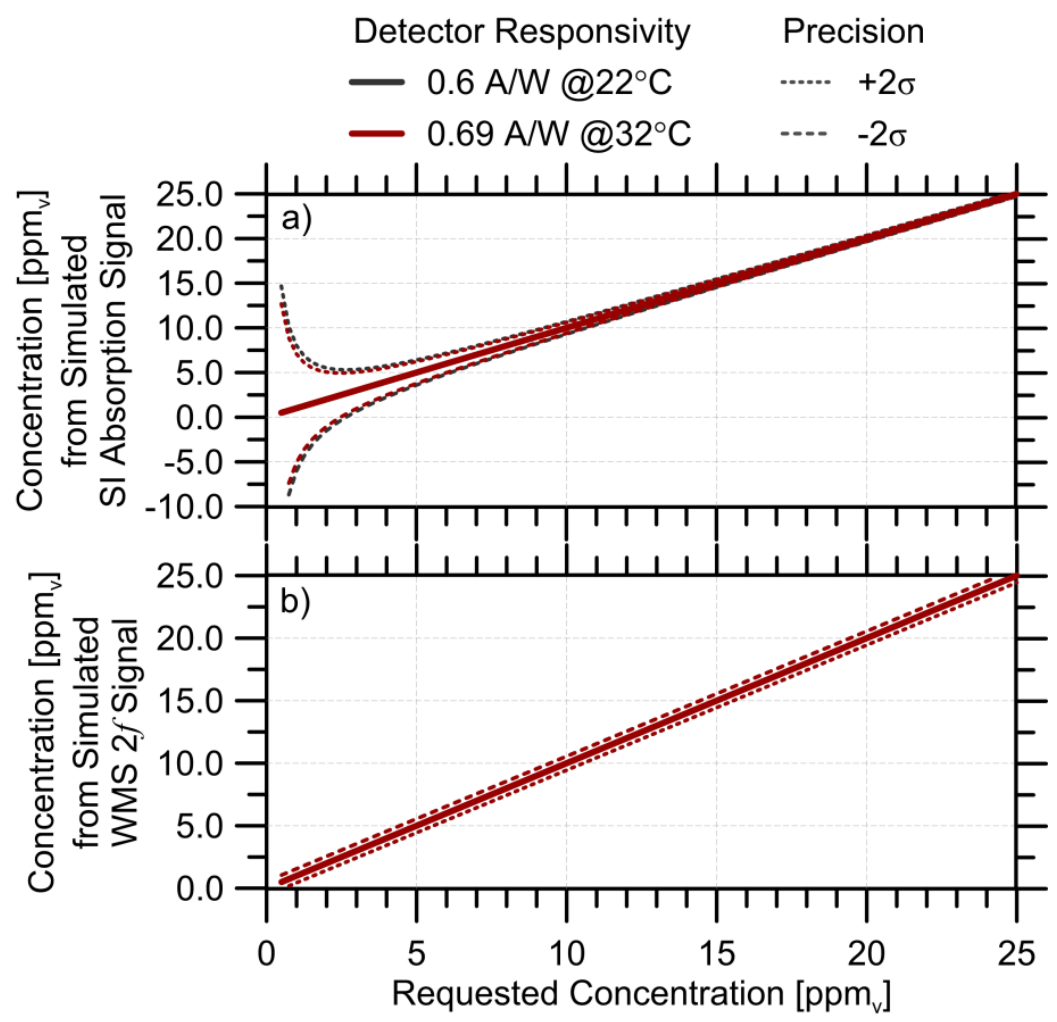

Figure 3.11: Simulated SI (a) and WMS (b) methane measurements, including the 95\% (2б) measurement precision for a signal averaged for 10 seconds. Computed at the $2 v_{3} \mathbf{R}(3)$ absorption manifold, at $23^{\circ} \mathrm{C}$ and $101.325 \mathrm{kPa}$, with a $5.79 \mathrm{~m}$ optical path-length, and experimentally-obtained laser and noise intensity characteristics

The same simulation was used to determine the influence of changes in detector responsivity from the time of calibration to the time of measurements. Simulations were performed assuming a worst-case photodetector responsivity of $0.6 \mathrm{~A} / \mathrm{W}$ (at $22^{\circ} \mathrm{C}$ ) at the time of calibration, and a photodetector responsivity of $0.69 \mathrm{~A} / \mathrm{W}\left(\right.$ at $32^{\circ} \mathrm{C}$ ) at the time of measurement when the methane VMR was calculated from absorption and $2 f$ features. The resulting SI and WMS measurement precision was nearly unchanged (less than a $0.001 \mathrm{ppm}_{\mathrm{V}}$ difference at $2 \mathrm{ppm}_{\mathrm{V}}$ ) when compared the precision differences mentioned above. This negligible change is attributable to the inherent ability for the signal processing methods used in this system to account for changes in mean return intensity.

To reduce the influence of detector responsivity, more expensive extended InGaAs detectors have since become available which possess a lower temperature dependence in the spectral operating range of this system at the expense of having an increased dark current response (Laser Components, 2015a). For a field-deployable 
solution it is suggested that thermal stabilization of the detectors or use of the extended InGaAs photodiodes should be used to limit the variability of responsivity with fluctuations in ambient temperature.

\subsubsection{Laser and Laser Control}

The lasers used in this setup were fiber-coupled narrow-bandwidth InGaAsP distributed feedback (DFB) diodes (NEL, NLK1U5EAAA) with an approximate spectral linewidth of $2.0 \mathrm{MHz}$ and a fiber output power of approximately $15 \mathrm{~mW}$ at a current of $120 \mathrm{~mA}$. These diodes were designed to be spectrally tuned within the $2 v_{3}$ R- and Q-branches of methane using thermal and current control, as illustrated in Figure 3.12. Table 3.3 lists the approximate laser controller temperatures and drive currents required to tune the lasers to these specific methane absorption manifolds.

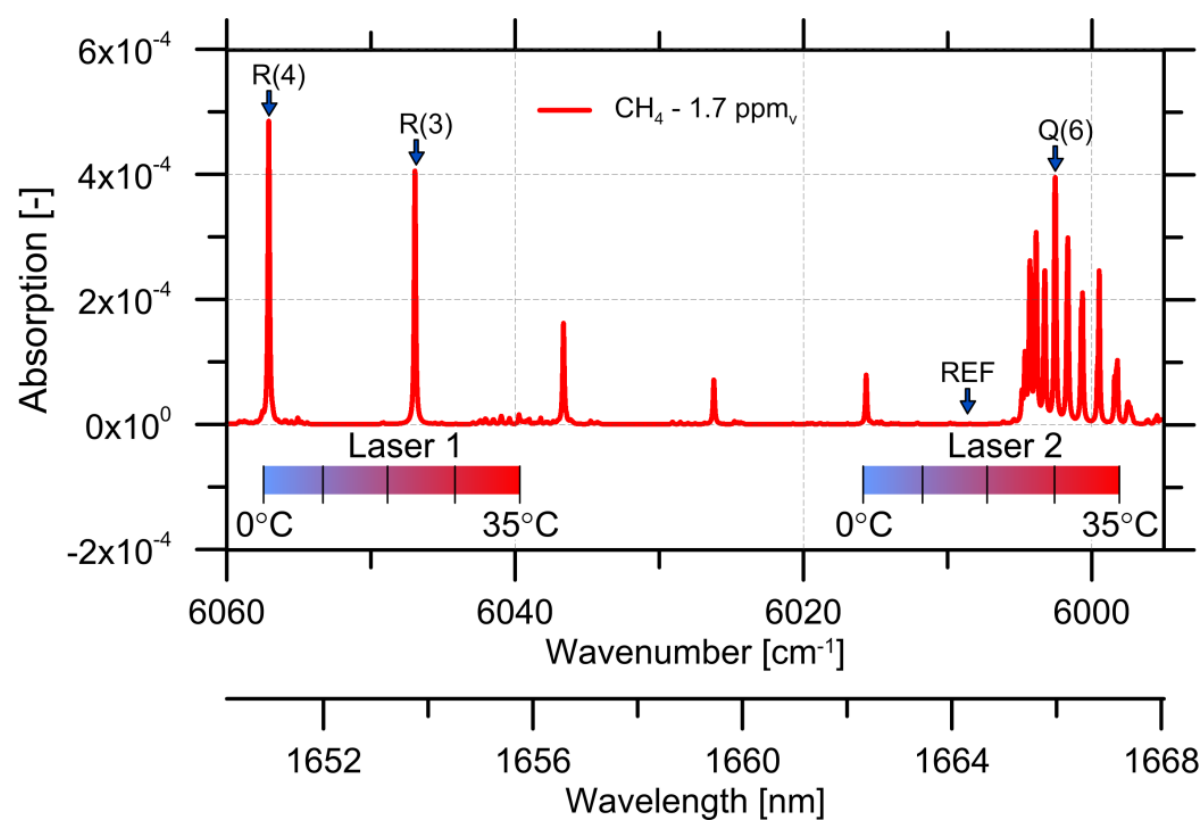

Figure 3.12: Thermal spectral tuning ranges of Laser 1 and Laser 2 
Table 3.3: Laser Diode Output Characteristics

\begin{tabular}{|c|c|c|c|c|c|}
\hline \multirow[b]{2}{*}{ Parameter } & \multirow[b]{2}{*}{ Units } & \multicolumn{2}{|c|}{ Laser 1} & \multicolumn{2}{|c|}{ Laser 2} \\
\hline & & $Q(6)$ & REF & $R(3)$ & $R(4)$ \\
\hline Approx. Wavelength & $\mathrm{nm}$ & 1665.96 & 1664.25 & 1653.73 & 1650.96 \\
\hline Approx. Wavenumber & $\mathrm{cm}^{-1}$ & 6002.55 & 6008.71 & 6046.94 & 6057.08 \\
\hline Spectral Linewidth & $\mathrm{MHz}$ & 2.0 & 2.0 & 2.0 & 2.0 \\
\hline Set Temperature & ${ }^{\circ} \mathrm{C}$ & 35.14 & 25.27 & 28.27 & 1.06 \\
\hline Set Current Offset & $\mathrm{mA}$ & 120.20 & 89.55 & 88.70 & 85.85 \\
\hline Temp. Tuning Rate & $\mathrm{nm} /{ }^{\circ} \mathrm{C}$ & 0.1 & 0.1 & 0.1 & 0.1 \\
\hline Current Tuning Rate & $\mathrm{pm} / \mathrm{mA}$ & $0.3-0.5$ & $0.3-0.5$ & $0.3-0.5$ & $0.3-0.5$ \\
\hline Output Power (fiber) & $\mathrm{mW}$ & $16 \pm 1.0$ & $11 \pm 1.0$ & $15 \pm 1.0$ & $15 \pm 1.0$ \\
\hline
\end{tabular}

The injection currents and diode temperatures were set by a pair of ILX Lightwave 3724C laser controllers, one for each laser diode. The controllers were configured to ensure safe start-up and operation within a set range of parameters, automatically limiting any settings or external inputs which may have led to damage of the control system or diodes. Additionally, compared to alternative control units, the $3724 \mathrm{C}$ has low output current and temperature drift characteristics, while permitting an external modulation at $\sim 20 \mathrm{~mA} / \mathrm{V}$. To limit the transfer of noise from the external modulation signals, the controllers employed an internal analog filter which restricts the maximum input modulation frequency to about $500 \mathrm{kHz}$.

\subsubsection{Optical References}

Route $\mathrm{C} 1$ in Figure 3.8 illustrates the laser reference route, which provided a means of monitoring a high SNR laser intensity for signal synchronization, component troubleshooting, and system calibration. Route C2 included a fixed-volume sealed quartz gas cell containing a known volume mixing ratio of nitrogen and methane at a fixed pressure, with specifications listed in Table 3.4.

Table 3.4: Fiber-Coupled Reference Cell Specifications

\begin{tabular}{l|c|c}
\hline \multicolumn{1}{c|}{ Parameter } & Units & Value \\
Volume Mixing Ratio $\left(\mathrm{CH}_{4}\right.$ in $\left.\mathrm{N}_{2}\right)$ & $\mathrm{ppm}_{\mathrm{v}}$ & $55,000 \pm 5,500$ \\
Pressure & bar & $1.0 \pm 0.025$ \\
Optical Path-Length & $\mathrm{mm}$ & $160 \pm 0.5$ \\
\hline
\end{tabular}

The gas cell and collimating optics were suspended within an aluminum enclosure, limiting misalignment. Figure 3.13 illustrates the reference cell, collimators, and pigtailed fibers. 


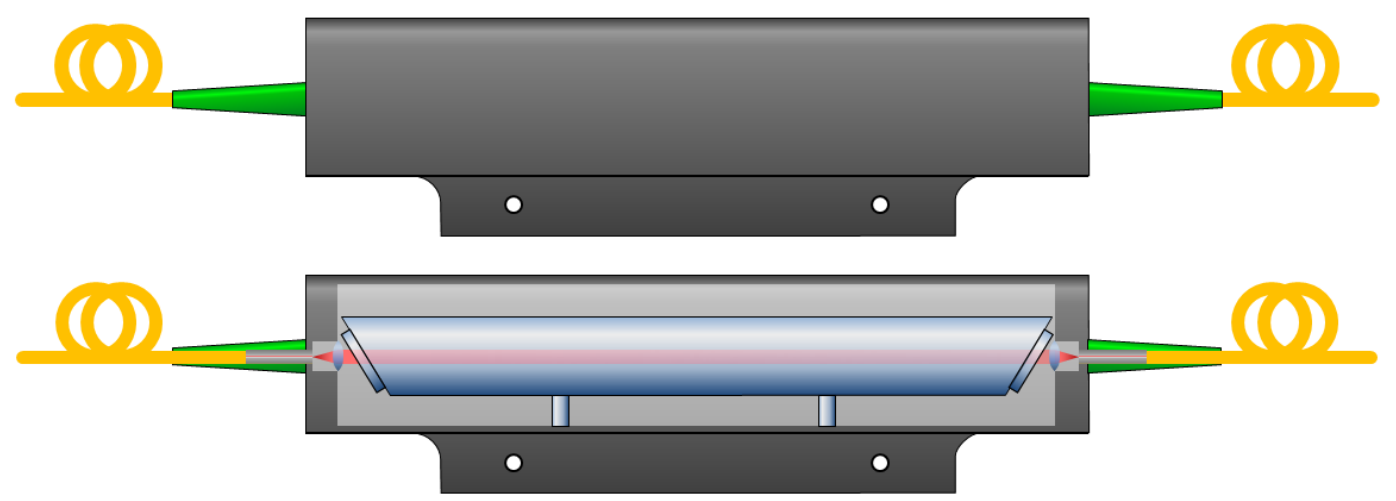

Figure 3.13: Schematic of fiber-coupled reference cell

The TDLAS system synchronously acquires SI or $2 f$-WMS signals from the optical sensor head (route C4) and the reference cell. Due to the potentially low signalto-noise ratio of the $2 f$ signal from the sensor head during measurements at low methane concentrations, the reference cell absorption or $2 f$ signal maximum was used to provide the time-index (and approximate wavenumber, $v_{p}$ ) at which the absorption manifold peak was traversed, and was subsequently used to infer an absorption or $2 f$ value from the remote optical sensor signal as illustrated for the WMS technique in Figure 3.14. The measured $2 f$ signal from the sensor head was then converted to a methane volume mixing ratio using either a theory-based or an experimental calibration as detailed in Section 4.6.

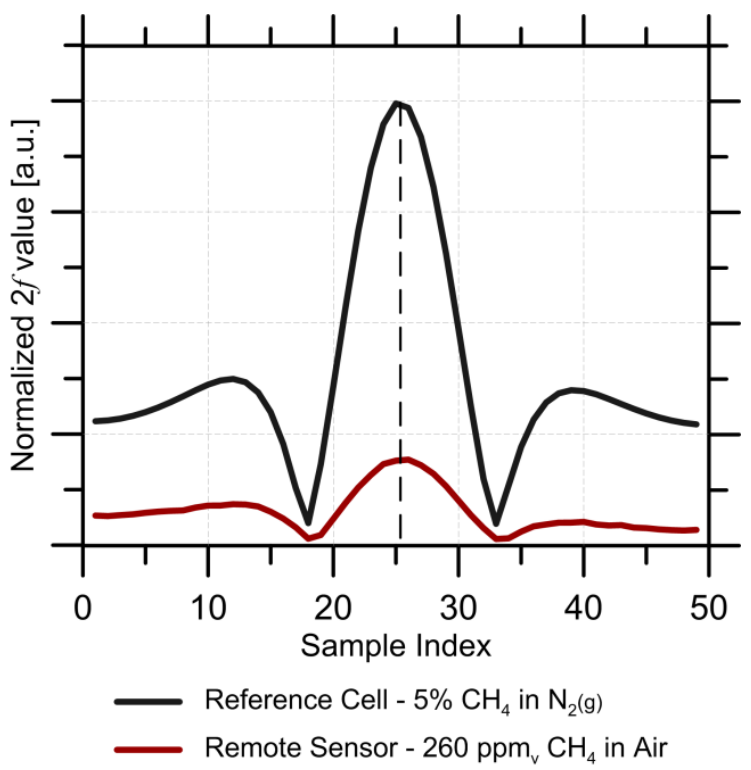

Figure 3.14: Inferred remote optical cell $2 f$ value from reference cell $2 f$ signal maximum 
The primary purpose of the reference cell was to provide a time-synchronized wavelength reference. The wavelength of the observed methane absorption peak was dependent on the methane VMR and pressure within the cell, and the optical path length of the interrogation region. Absorption was simulated to determine the extremes of the inferred peak shift on measured methane VMR. The simulation was conducted using a Lorentzian profile with $p=1$ bar \pm 0.05 bar, methane volume mixing ratio $=55,000 \mathrm{ppm}_{\mathrm{v}} \pm 5,500 \mathrm{ppm}_{\mathrm{v}} \mathrm{CH}_{4}$ in $\mathrm{N}_{2}$, and path-length $=16.0 \mathrm{~mm} \pm 1.0 \mathrm{~mm}$. As shown in Figure 3.15, in the most extreme case, the difference in the wavelength of the peaks is less than $0.0015 \mathrm{~nm}$. The simulation suggests that when the reference cell is used to infer the time at which the methane manifold peak has been traversed in the remote optical sensor, the result is a negligible maximum inferred methane volume mixing ratio error of $5 \times 10^{-6}$ at $2.0 \mathrm{ppm}_{\mathrm{v}}$ and $3 \times 10^{-4} \mathrm{ppm}_{\mathrm{v}}$ at $200 \mathrm{ppm}_{\mathrm{v}}$.
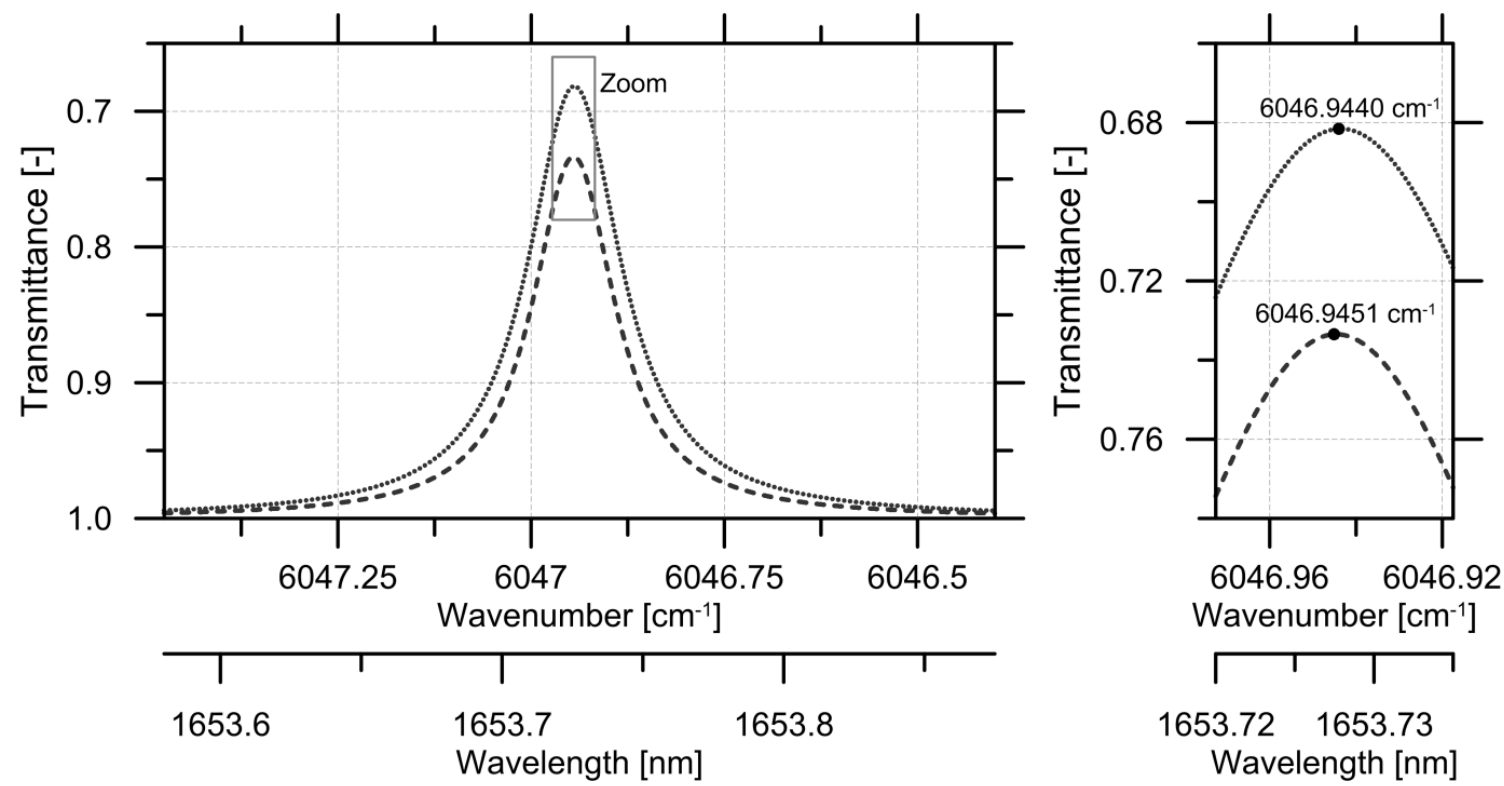

Figure 3.15: Extremes of the simulated transmittance through the fiber-coupled reference cell for the methane $2 v_{3} R(3)$ absorption manifold, calculated using Lorentzian profiles and the $95 \%$ uncertainties from the reference cell specifications ( $p=1$ bar \pm 0.05 bar, methane volume mixing ratio $=55,000 \mathrm{ppm}_{\mathrm{v}} \pm 5,500 \mathrm{ppm}_{\mathrm{v}} \mathrm{CH}_{4}$ in $\mathrm{N}_{2}$, and path-length $=16.0 \mathrm{~mm}$ $\pm 1.0 \mathrm{~mm})$.

\section{Fiber-ring Resonator}

A fiber-ring resonator (FRR) is a passive device that is used to accurately measure the change in a time-varying wavelength by observing optical interference fringes, with 
similar functionality to a free-space etalon. In this system, the input to the FRR was tapped directly off the active laser (as illustrated in the system diagram in Section 3.1.3), and provided a means of obtaining a time-synchronized measurement of the modulated laser output wavelength. A fluctuating wavelength input to the FRR results in a varying intensity output signal comprised of optical interference fringes, as illustrated in Figure 3.16 .

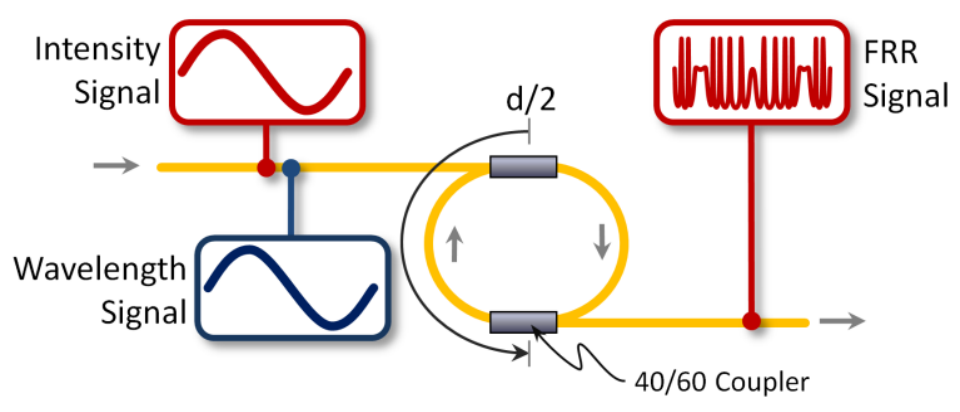

Figure 3.16: Schematic of the fiber-ring resonator (FRR), with an input signal of varying intensity and wavelength, and the resultant output interference signal

The distance between the interference fringes is known as the free spectral range, $F S R=c / d n[\mathrm{~Hz}]$ and is governed by the speed of light, the fiber index of refraction $(n)$ and the physical length of the fiber-ring (the combined ring length including the two 40/60 fiber couplers, $d[\mathrm{~m}]$ ). The fiber-ring resonator FSR was determined using the absolute wavelength of the easily-recognizable absorption feature peaks of the $\mathrm{Q}(3)$ Q(6) $2 v_{3}$ near-IR methane manifolds. To observe these features within a single wavelength sweep, the laser was held at a constant temperature and modulated with a high-amplitude low-frequency sinusoidal injection current. Simultaneous reference cell and FRR signals ( $\mathrm{C} 2$ and $\mathrm{C} 3$ legs respectively) were then acquired and averaged for 5 seconds, as plotted in Figure 3.17. 


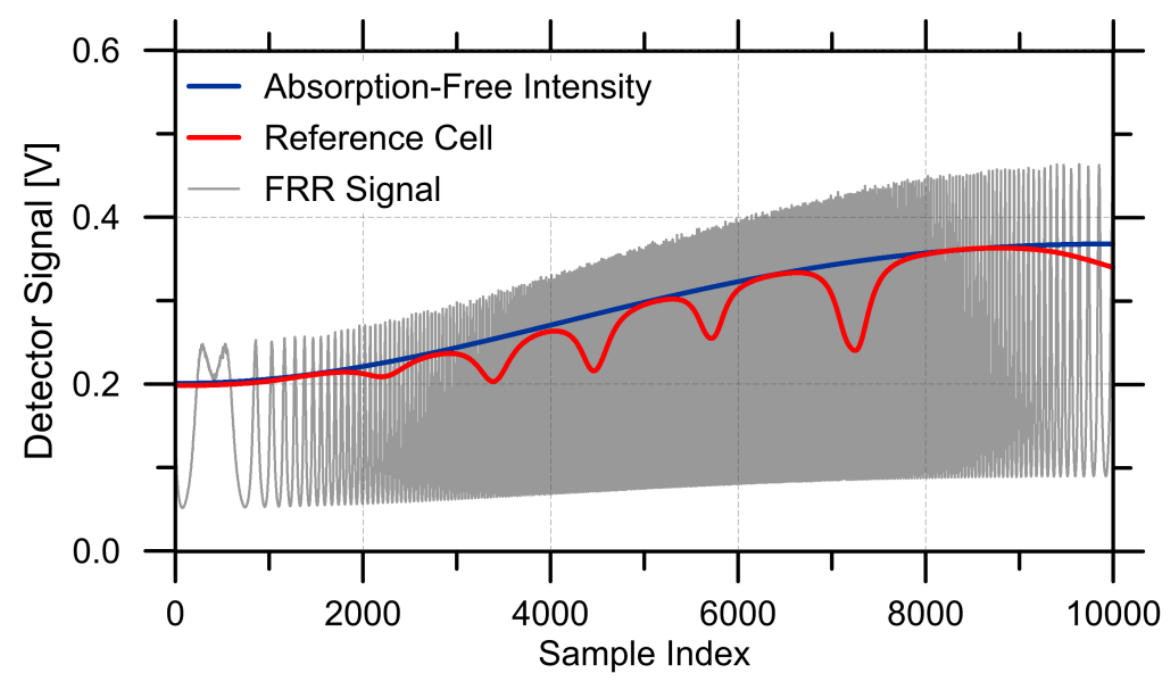

Figure 3.17: Raw FRR calibration signals computed using the $2 v_{3}$ Q-branch for the methane reference cell containing 5.5\% $\mathrm{CH}_{4}$ in $\mathrm{N}_{2}$ at a pressure of 1.0 bar and an optical path-length of $16 \mathrm{~cm}$.

To estimate the reference cell absorption signal the pre- and post-absorption intensity signals are required. The post-absorption intensity signal is obtained directly from the return signal photodetector, while the pre-absorption intensity signal must be estimated. In this case, the absorption-free reference cell intensity was determined using a Nelder-Mead optimization ${ }^{1}$, which minimized the residual error between the reference cell signal and theory-based Voigt and Lorentzian based absorption profiles for the observed absorption features. The resultant best-fit between the theoretical and experimental absorption signals is illustrated in Figure 3.18.

The absorption feature peaks in Figure 3.18 were used to provide absolute wavelength references, from which the fiber-ring resonator FSR was derived. The FSR was estimated from the quotient of the spectral distance and the number of interference peaks between each absorption feature, resulting in an average fiber-ring resonator FSR of $0.3459 \mathrm{GHz}\left(0.01153 \mathrm{~cm}^{-1}\right)$, illustrated in Figure 3.19 .

\footnotetext{
${ }^{1}$ Nelder-Mead optimization between experimental and theoretical Q-branch absorption data, using 9 points (between 6002.85 and $6004.1 \mathrm{~cm}^{-1}$ ), at 4 different cell temperatures $(294.28,297.50,302.15$, and $304.77 \mathrm{~K}$ ).
} 


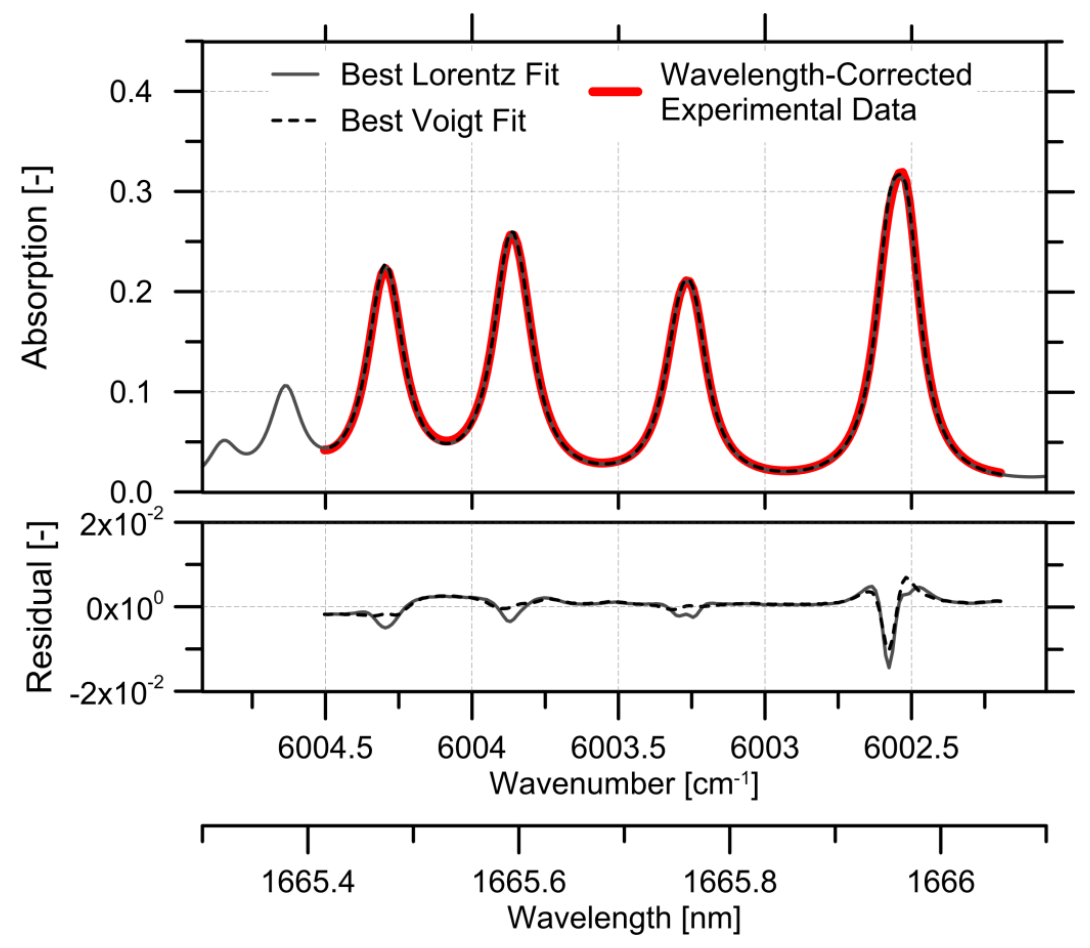

Figure 3.18: Best-fit of the simulated and experimental reference cell absorption signals for the $Q(3)-Q(6)$ (left to right) methane absorption manifolds within the near-IR $2 v_{3}$ branch.

The reference cell contained $5.5 \% \mathrm{CH}_{4}$ in $\mathrm{N}_{2}$ at a pressure of 1 bar and an optical pathlength of $16 \mathrm{~cm}$.

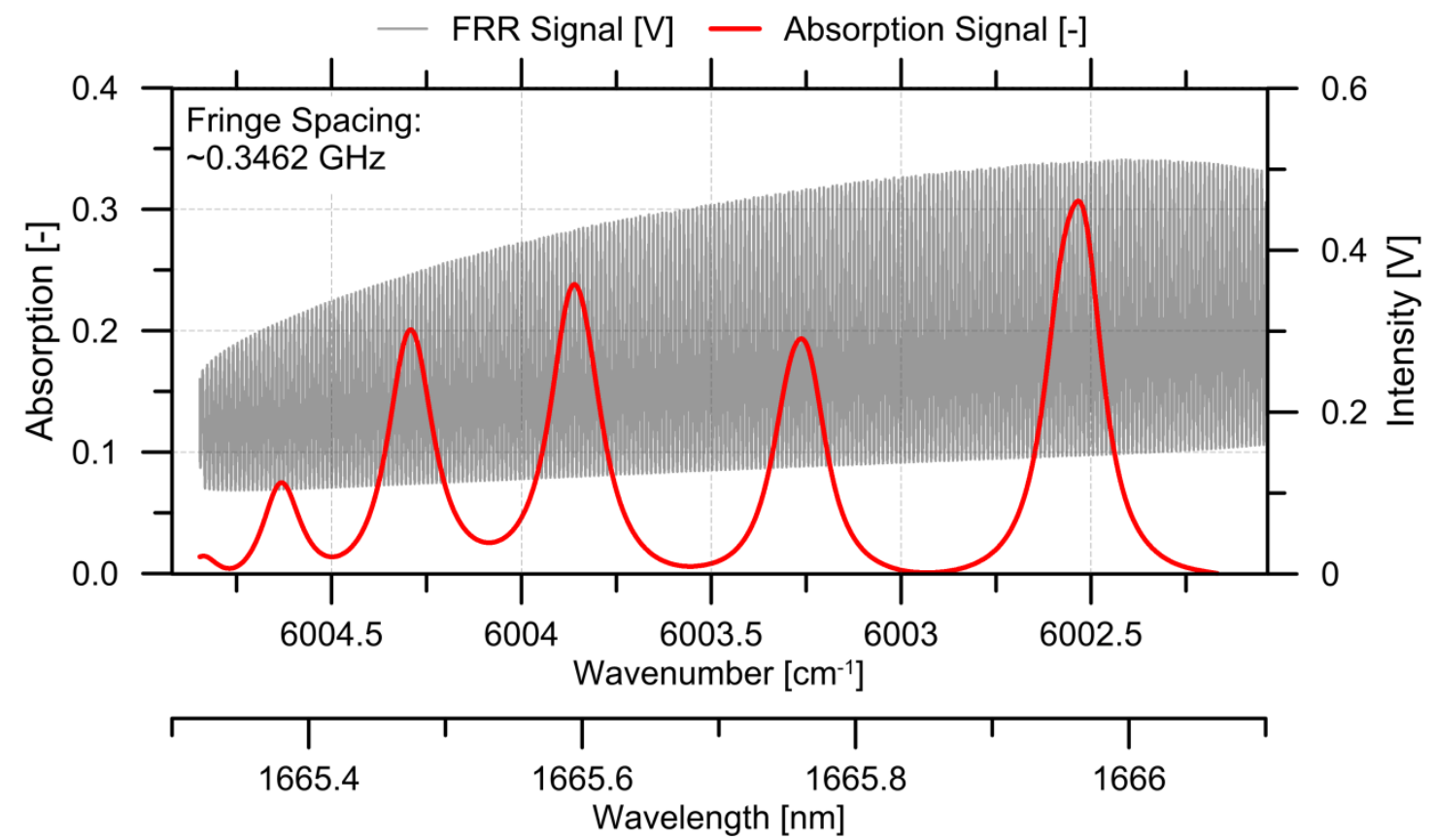

Figure 3.19: FRR calibration signals; linearized FRR signal (grey), and absorption signal (red) from the reference cell containing 5.5\% $\mathrm{CH}_{4}$ in $\mathrm{N}_{2}$ at a pressure of 1.0 bar and an optical path-length of $16 \mathrm{~cm}$. 


\subsubsection{Control System}

A National Instruments PXI system provided a common software-controllable platform that integrated the wide range of hardware components necessary to monitor and control the TDLAS system. Figure 3.20 shows a schematic of the PXI chassis, integrated components, peripheral equipment, and connections.

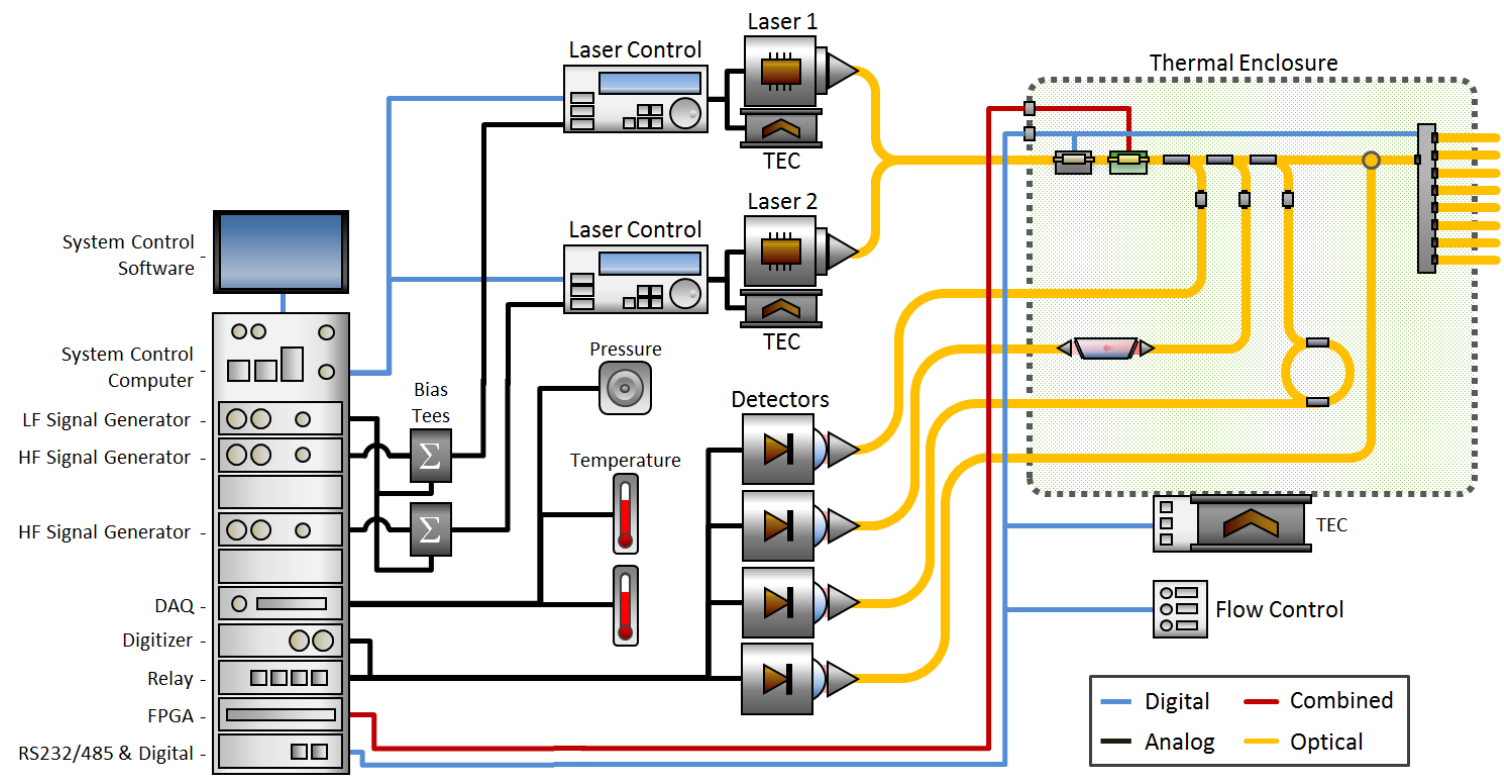

Figure 3.20: Control system routing diagram. LF and HF denote the low-frequency sweep and the high-frequency modulation signal generators respectively.

\subsubsection{Analog to digital hardware}

The data acquisition side of the TDLAS system can be separated into support and optical signals. Support signals included temperature, pressure, and voltage monitoring, which were sampled at 14-bit resolution at a nominal rate of $1 \mathrm{kHz}$ via a National Instruments PXIe-6363 data acquisition card (DAQ). These signals were used for diagnostics, monitoring, measurement-error estimations, and system calibration. To measure optical signals from the photodetectors, a 16-bit NI PXIe-5922 digitizer was used. This device had an excellent frequency response over the typical TDLAS operating range, and could simultaneously acquire data at up to $1.5 \mathrm{MS} / \mathrm{s}$ on two channels. The four detectors $(\mathrm{C} 1-$ C4, Figure 3.8) were multiplexed to the PXIe-5922 digitizer using a PXIe-2548 RF relay. The relay eliminated the need for multiple digitizers, and improved system stability by eliminating the need to interchange electrical connections for different system 
configurations. Table 3.5 lists the digitized analog inputs and associated sample rates used in the TDLAS system. The effective digitization resolution for the pressure transducer and thermistors are discussed further in Section 3.1.4.3.

Table 3.5: Analog Signal Digitization Specifications

\begin{tabular}{l|c|c|c|c}
\hline Analog Signal Source & $\begin{array}{c}\text { Digitization } \\
\text { Device }\end{array}$ & $\begin{array}{c}\text { Sample Rate } \\
{[\mathrm{kHz}]}\end{array}$ & $\begin{array}{c}\text { Digitization } \\
\text { Range }[\mathrm{V}]\end{array}$ & $\begin{array}{c}\text { Resolution } \\
{[\mathrm{mV}]}\end{array}$ \\
\hline Thermistors & PXle-6363 & 1.0 & 5.0 & 0.3 \\
Pressure Transducer & PXle-6363 & 1.0 & 5.0 & 0.3 \\
InGaAs Detectors & PXle-5922 & $100.0-1,500.0$ & 0.5 & $7 \times 10^{-3}$ \\
\hline
\end{tabular}

\subsubsection{Signal Generation}

Two 16-bit National Instruments PXI-5406 signal generators were used to generate the laser driving signals. These signal generators were selected based on their ability to produce high-resolution, high-voltage sinusoids, with relatively ${ }^{2}$ low passband flatness over the intended operating range of the TDLAS system. To ensure proper synchronization, the NI-5406 signal generators were internally clocked with a $100 \mathrm{MHz}$ signal, and synchronized with each other and the digitizer using a $10 \mathrm{MHz}$ clock from the PXI chassis controller.

Signals from two signal generators were combined via a bias tee to form the laser driving signal instead of using a single arbitrary waveform generator. The Picosecond 5543 bias tee was selected for its flat frequency response and bandwidths of DC to $5 \mathrm{kHz}$ and 0.25 to $55,000 \mathrm{MHz}$ on the low- and high-frequency inputs respectively. Combining the sweep and modulation frequencies from two dedicated signal generators improved the overall resolution of the laser driving signal, and permitted safe and seamless switching of signal amplitude and frequency (necessary for switching between SI and WMS methods) without the need to reconfigure the signal generation hardware.

\footnotetext{
${ }^{2}$ As compared to available alternatives such as the National Instruments 5422, a 16-bit amplified arbitrary waveform generator
} 


\subsubsection{Temperature and Pressure Measurements}

The laser control system, thermally-stabilized enclosure, and test chamber temperatures were measured using biased thermistors monitored using the PXIe-6363 DAQ. The laser controllers, laser diodes, TEC, and power supply modules had internal thermistors that sent digital temperature data to the control system for external monitoring and datalogging. Ambient pressure was measured using an Omega PX2760 pressure transducer, and monitored using the PXIe-6363 DAQ. Specifications on the thermistors and pressure sensor are shown in Table 3.6.

Table 3.6: Atmospheric Sensing Component Specifications

\begin{tabular}{l|c|c|c}
\hline \multicolumn{1}{c|}{ Parameter } & $\begin{array}{c}\text { Pressure } \\
\text { Transducer }\end{array}$ & $\begin{array}{c}\text { Air Temperature } \\
\text { Thermistor }\end{array}$ & $\begin{array}{c}\text { Surface-Mount } \\
\text { Thermistor }\end{array}$ \\
\hline Model & $\mathrm{PX} 2760$ & $\mathrm{ON}-406$ & $\mathrm{ON}-930$ \\
Accuracy & $\pm 0.25 \%$ & $\pm 0.1^{\circ} \mathrm{C}$ & $\pm 0.1^{\circ} \mathrm{C}$ \\
Input Range & $0.8-1.1 \mathrm{bar}$ & $-15-50^{\circ} \mathrm{C}$ & $-15-50^{\circ} \mathrm{C}$ \\
Output Range & $0.1-5.1 \mathrm{~V}$ & $0.01-2.5 \mathrm{~V}$ & $0.01-2.5 \mathrm{~V}$ \\
Digitization Resolution & $2.0 \mathrm{~Pa}$ & $0.0075^{\circ} \mathrm{C}$ & $0.0075^{\circ} \mathrm{C}$ \\
\hline
\end{tabular}

\subsection{Test chamber}

To accurately quantify the performance of the TDLAS system and associated optical sensor head, a test chamber was constructed to immerse the measurement cell in a uniform and well-mixed plume of methane at controllable concentrations. The test chamber ( $2 \mathrm{~m}$ long, $0.295 \mathrm{~m}$ inner-diameter PVC tube) consisted of an injection section, a flow conditioning section, and a test section as illustrated in Figure 3.21.

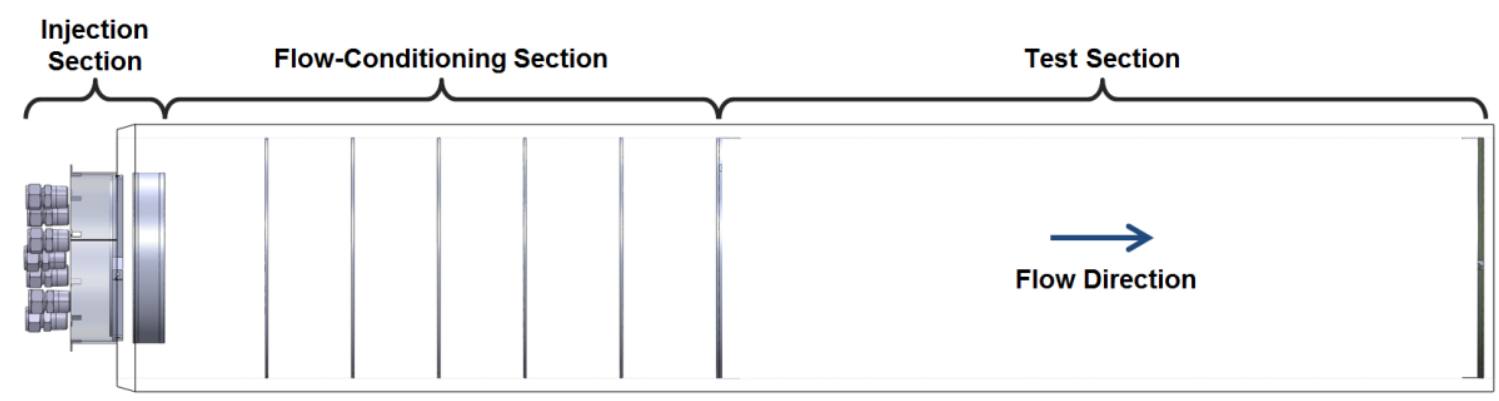

Figure 3.21: Test chamber cross-section 
The injection section directed the pre-mixed gas from the injection fittings through two perforated plates (with hole diameters of 1/8", and an open area of about $25 \%$ ) to ensure complete mixing and the break down the input flow, as illustrated in Figure 3.22. Downstream of the perforated plates are two rigid mesh $(8 \times 8$ mesh with a $74 \%$ open area, 0.017 " wire diameter) disks, separated by $30 \mathrm{~mm}$ of tightly-packed $2 \mathrm{~mm}$ diameter beads, which were used to prevent any eddies larger than one bead diameter from passing downstream. The pressure drop across the beads and mesh encouraged uniform flow across the inlet of a diffuser.

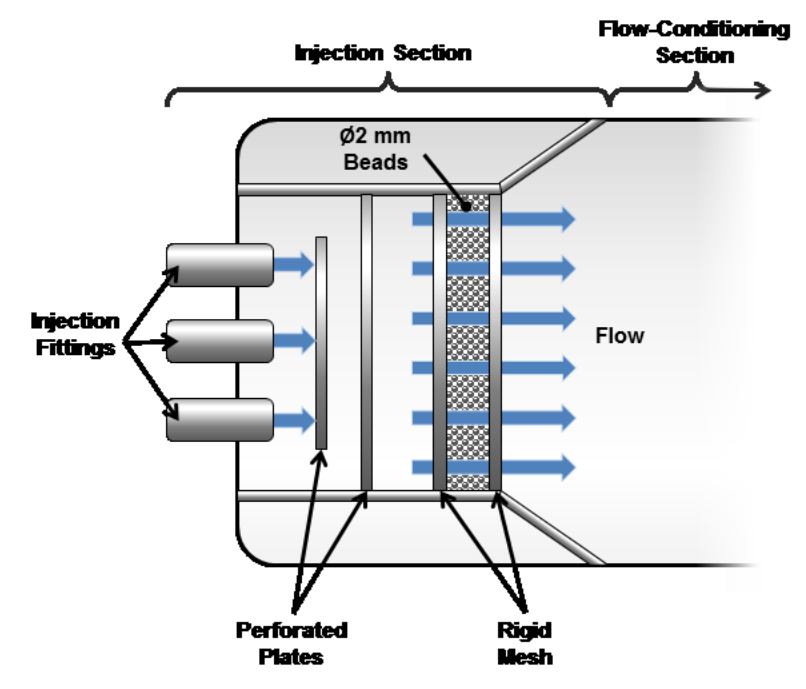

Figure 3.22: Illustrated cross-section of the test chamber injection section

As shown in Figure 3.22, the injection section fed into a $0.75 \mathrm{~m}$ long flow conditioning section, which consisted of six equally-spaced perforated plates (with hole diameters of 0.25 " and an open area of $12 \%$ ) designed to encourage uniform flow. Various mass-flow controller combinations were used in the gas mixing system (Appendix A) to achieve volume mixing ratios in the test section between 2 and $200 \mathrm{ppm}_{\mathrm{v}}$ at flow rates between 50 and 500 SLPM (mean flow velocities within the test section between 0.012 and $0.116 \mathrm{~m} / \mathrm{s}$ ). A mass flow rate of 400 SLPM (average velocity of $0.093 \mathrm{~m} / \mathrm{s}$ ) was used for most of the results presented herein, except for long runs at methane concentrations $>50 \mathrm{ppm}_{\mathrm{v}}$, for which the flow rate was halved (200 SLPM, $0.047 \mathrm{~m} / \mathrm{s}$ ) to conserve calibration gas. 
Downstream of the flow conditioning section was a $1 \mathrm{~m}$ long test section. To prevent laboratory air from entering the test section a lightweight perforated plastic bag was taped over the end of the pipe. Figure 3.23 illustrates the different test section configurations for quantifying the performance of various optical sensor head configurations. The 5.79 meter multipass fiber-coupled optical sensor head was suspended in the centre of the tube, supported by aluminum struts $(4.25 \mathrm{~cm}$ wide, $1.5 \mathrm{~mm}$ thick, with the rounded thin edge normal to the flow), allowing the interrogation region to be fully immersed in the flow. Other tested optical configurations (discussed in Section 3.1.2) were mounted onto an optical bread-board, with posts holding the optical components at the centre of the test-chamber, as illustrated in Figure 3.23b. An Omega ON-406 thermistor (specifications listed in Section 3.1.4.3) measured temperature within the test section, and was suspended just outside of the optical interrogation region within the optical sensor head. Further details on the performance of the test system are provided in Appendix A.

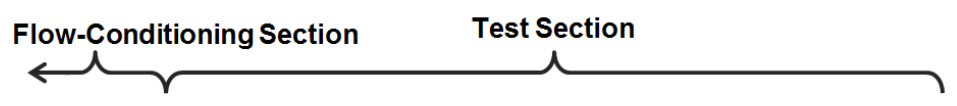

a) 5.79 m Optical Sensor Head Configuration

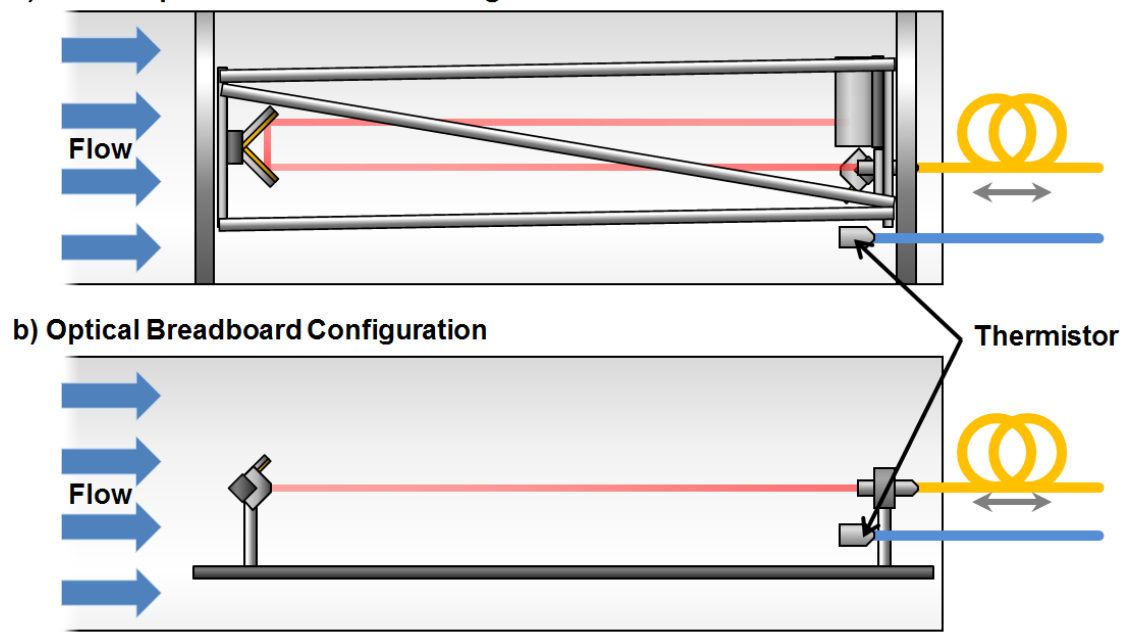

Figure 3.23: Test-section configurations; a) $5.79 \mathrm{~m}$ remote optical sensor, and b) component configuration test board. Note the flow direction and location of the thermistor 


\subsubsection{Mechanical Jitter}

It was observed that when seemingly trivial vibrations were introduced to the structure supporting the test chamber (in the form of a keyboard typing, printer printing, computer fan running, a person walking past, etc.) the standard deviation of the measured absorption or $2 f$ value was generally reduced. This behaviour was attributed to mechanical averaging of optical interference fringes produced by interacting reflections from optical surfaces within the sensor head. Optical interference occurs when reflected light rays and the original laser beam are coupled together onto the photodetector, causing the measured intensity to vary relative to the phase-difference, $\theta$ [radians], between the light rays,

$$
\theta=n \frac{4 \pi}{\lambda} \varphi
$$

where $\varphi[\mathrm{m}]$ is the distance between the reflective surfaces. The result is a wavelengthdependent intensity fluctuation (i.e. fringes) unrelated to molecular absorption, which leads to artificial variations in the measured absorption and $2 f$ signals (Cassidy and Reid, 1982a; Bomse et al., 1992; Cvijin et al., 1992; Persson et al., 2007; Rieker et al., 2009; Chakraborty et al., 2010).

It is well established that optical fringes can be supressed by 'jittering' the relative distance between the surfaces causing the optical interference, at an amplitude greater than $0.5 \lambda$ and a period shorter than the measurement averaging time period. If the rateof-change in $\varphi$ (a function of the jitter amplitude and frequency) is sufficient, the intensity variation of multiple fringe states will be included within one measurement period, effectively averaging out the artificial variations in return intensity and reducing the perceived variation in measured methane volume mixing ratio (VMR). This technique has been demonstrated to reduce the minimum detectable absorbance of various gas species by up to a factor of 10, approaching the theoretical detection limit of $10^{-6}$ or $10^{-7} \mathrm{~cm}^{-1}$ (Bomse et al., 1992; Hu et al., 1993; Persson et al., 2007; Andersson et al., 2007). 
Due to the highly idealized nature of the laboratory setup, preliminary results suggested that disturbances normally considered inconsequential (i.e. via transient vibrations as noted above, atmospheric refraction due to turbulence, operation of the test chamber at different flow rates etc.) were perceptible in the TDLAS-based measurement system. Relative to the low-vibration background, these minor disturbances were capable of introducing subtle changes in $\varphi$, sufficient to reduce measurement deviation at averaging times of $0.1 \mathrm{~s}$ or longer. This sensitivity of the measured methane VMR standard deviation (with both the SI and WMS methods) was problematic for obtaining repeatable measurements of system performance. More specifically, to permit quantitative comparisons of various measurement system configurations and software processing algorithms (as presented in detail in Chapter 4 and Chapter 5), it was essential to ensure comparative experiments were performed at directly comparable and reproducible conditions.

Since complete isolation of the sensor head from external disturbances would be nearly impossible to achieve, and would be inconsistent with an industrial environment (such as upstream oil and gas industry facilities that are the intended application target of the developed TDLAS technology), the choice was made to introduce a small but consistent amount of ambient vibrational disturbance to the lab setup. This was accomplished by placing a small computer fan on the table supporting the test chamber which was sufficient to subjugate any mechanical vibrations introduced by transient sources. The fan was located approximately $60 \mathrm{~cm}$ from the centerline of the test chamber and $50 \mathrm{~cm}$ from the opening of the test section, and was orientated such that the resultant airflow was directed away from the test chamber, as illustrated in Figure 3.24. The fan was a ball-bearing NMB 4715MS AC, which has a flow rate of approximately $50 \mathrm{cfm}$ at a fan speed of $\sim 1450 \mathrm{rpm}$, and a mass of $0.55 \mathrm{~kg}$. 


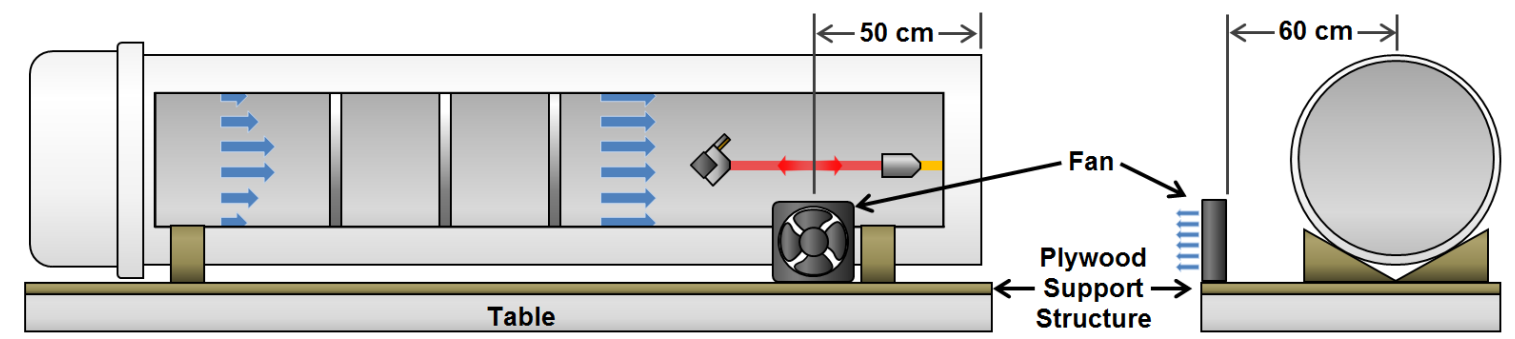

Figure 3.24: Fan location relative to exhaust end of test chamber. Note the location of the fan on the plywood support structure and the direction of the airflow, away from the test chamber.

Selected experiments were performed to quantify the influence of the fan on measurements. Figure 3.25 illustrates the influence mechanical vibration had on the standard deviation $(1 \sigma)$ of measured methane absorption and $2 f$ values at ambient methane concentrations $\left(\sim 1.7 \mathrm{ppm}_{\mathrm{v}}\right)$ over a range of averaging times. These tests were conducted with no flow (covered test chamber outlet) at ambient pressures and temperatures using the $2 v_{3} \mathrm{R}(3)$ methane manifold, and a $6 \mathrm{~m}$ optical fiber between the base station and the $5.79 \mathrm{~m}$ reflected path optical sensor head.
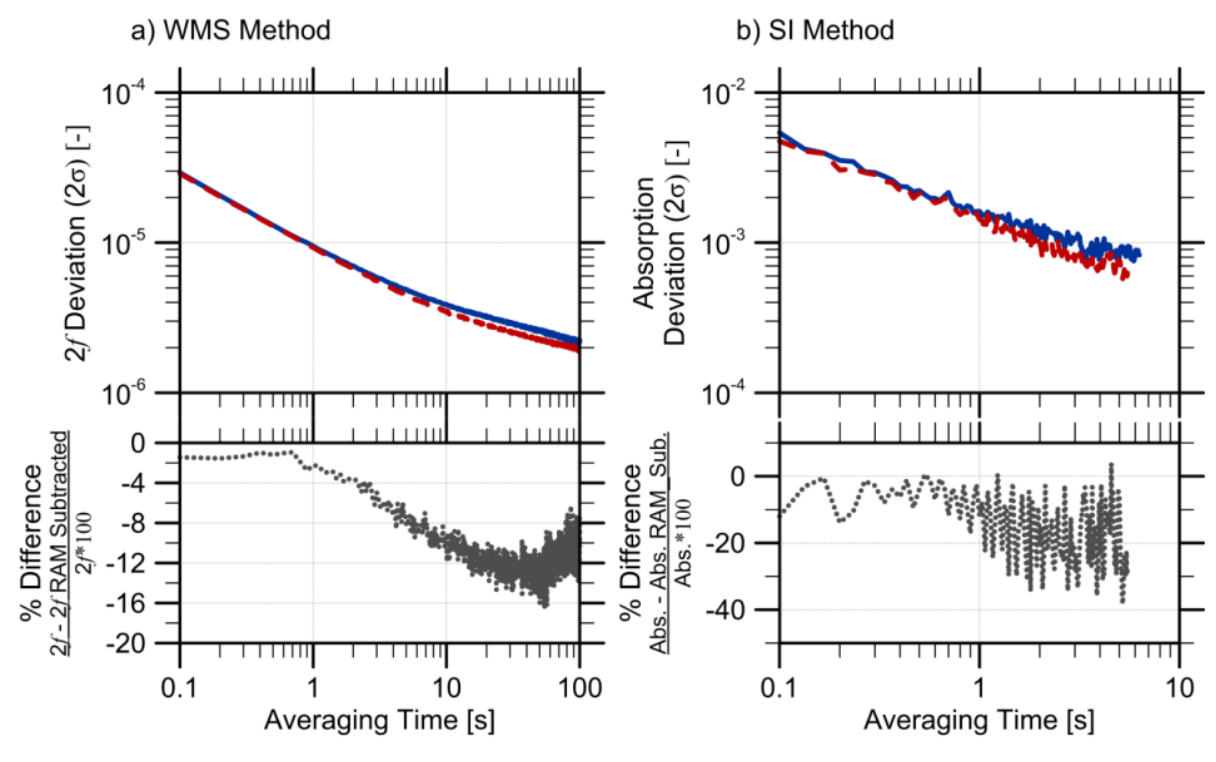

— No Jitter - - With Jitter

$\%$ Difference

Figure 3.25: Absorption and $2 f$ value standard $1 \sigma$ deviation (a) at the $2 v_{3} \mathbf{R}(3)$ methane manifold peak with (red) and without (blue) the computer fan, and (b) the difference between the deviation with and without the fan. Data acquired using a $6 \mathrm{~m}$ optical fiber between the base station and $5.79 \mathrm{~m}$ sensor head and at ambient pressures and temperatures. 
These results indicate that at a $1 \mathrm{~s}$ averaging time, the placement of the fan adjacent to the test cell caused a $25 \%$ reduction in the standard deviation of the measured absorption feature for the SI technique, and a $2.5 \%$ reduction in the standard deviation of the measured $2 f$ feature for the WMS technique. This equates to a reduction in measured methane VMR standard deviation $(2 \sigma)$ from $1.49 \mathrm{ppm}_{\mathrm{v}}$ to $1.35 \mathrm{ppm}_{\mathrm{v}}$ (reduction of $9.49 \%$ ) for the SI method, and from $1.58 \mathrm{ppm}_{\mathrm{v}}$ to $1.57 \mathrm{ppm}_{\mathrm{v}}$ (reduction of $0.75 \%$ ) for the WMS method (for experiments at ambient concentrations, $\sim 1.7 \quad \mathrm{ppm}_{\mathrm{v}}$ ). For measurements at higher concentrations, the effects of fringes on the larger absorption or $2 f$ values are less significant, and the reduction in standard deviation of the measured methane VMR would be even smaller. By introducing a fixed and consistent level of small-amplitude ambient vibration, it became possible to make direct and repeatable comparisons among different experiments. This was essential for making quantitative comparisons of the relative performance of different hardware and software changes on measurement performance, with a negligible influence on the absolute system performance as presented in Chapter 5. 


\section{Chapter 4 Signal Processing and Control}

This chapter presents the basic methods of control, acquisition, processing, and analysis used to process sweep integration (SI) and wavelength modulation spectroscopy (WMS) signals as part of quantifying measurement accuracy, precision, and lower detection limit.

\subsection{Data Acquisition}

The fiber-coupled photodetectors (discussed in Section 3.1.3.2) were operated in photovoltaic mode to maximize frequency response and signal resolution. The acquisition rate was selected based on the sweep and modulation frequencies and the fidelity required to sufficiently resolve the absorption or harmonic feature. Generally, the sample rate for:

- SI must be set at an integer multiple of the sweep-frequency to ensure equivalent samples for each sweep period, and

- WMS must be an integer multiple of both the sweep and modulation frequencies to ensure an integer number of points in the sweep and modulation periods.

For both the SI and WMS techniques, the minimum sample rate was determined by oversampling photodetector outputs and down-sampling the resultant digital signals to determine the sample rate at which the average absorption and second harmonic peak heights for the 5.79 meter multipass interrogation cell (at $\sim 2.0 \mathrm{ppm}_{\mathrm{v}}$ and $100 \mathrm{ppm}_{\mathrm{v}}$ ) deviated from the values obtained at the maximum acquisition rate of the digitizer $(10 \mathrm{MHz})$. Results of this testing are plotted in Figure 4.1. The peak heights of the measured absorption and second harmonic signals in the cell were extracted at the index of the maximum reference cell absorption- or $2 f$-peak signal. 

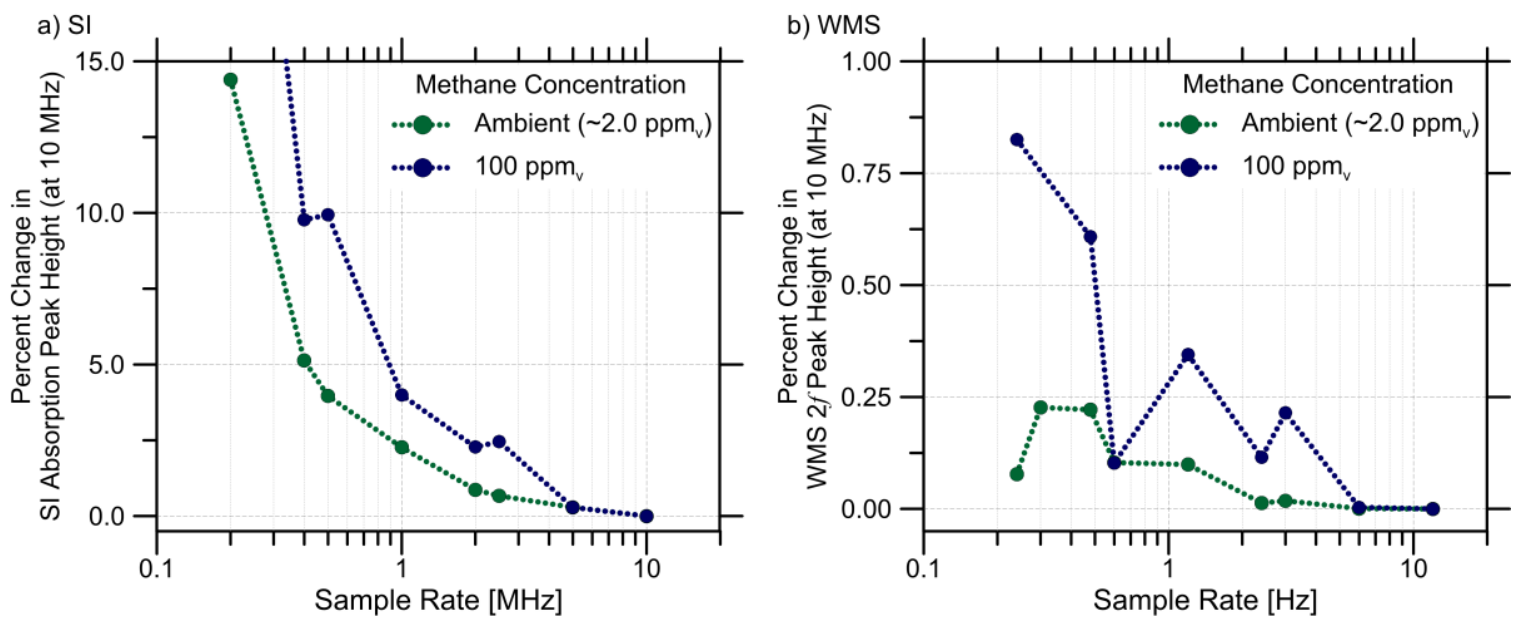

Figure 4.1: Effect of sample rate on measured interrogation cell absorption and $2 f$ values at the optimum SI and WMS laser modulation signals. Data acquired at ambient pressures and temperatures with the $5.79 \mathrm{~m}$ multipass sensor head.

The sample rate has a drastic effect on the measured absorption for the SI technique (note the difference in scales of the vertical axes on Figure 4.1a and b). Although sensitivity of the measured absorption value to sample rate decreases as the rate approaches $10 \mathrm{MHz}$ (the maximum rate of the data acquisition system), the plots suggest that sample rates near the system maximum are required to accurately determine the height of absorption peak with the SI technique. To permit real-time processing and manageable file sizes, a sample rate of $4.0 \mathrm{MHz}$ was selected for the SI technique (corresponding to a data acquisition rate of $64 \mathrm{MB} / \mathrm{s}$ ), which produced peak height results within $1 \%$ of those obtained at $10 \mathrm{MHz}$. For the WMS technique the measured second harmonic value was much less sensitive to sample rate. For sample rates above $500 \mathrm{kHz}$, the measured $2 f$ peak height was within $1.0 \%$ of the value obtained at the maximum sample rate of $\sim 10 \mathrm{MHz}$. Based on this result, a WMS sample rate of $0.4 \mathrm{MHz}$ was used in the TDLAS system, corresponding to a data acquisition rate of $5.6 \mathrm{MB} / \mathrm{s}$.

\subsection{Signal Analysis}

The raw digitized SI and WMS signals included noise contributions of varying frequency and amplitude from multiple sources (Werle et al., 1994) in addition to contributions due to absorption by methane. Figure 4.2 and Figure 4.3 demonstrate the influence of these noise sources by plotting typical SI and WMS photodetector signals (for test chamber 
methane volume mixing ratios of $100 \mathrm{ppm}_{\mathrm{v}}$ at ambient pressure and temperature) in the frequency domain ${ }^{3}$.
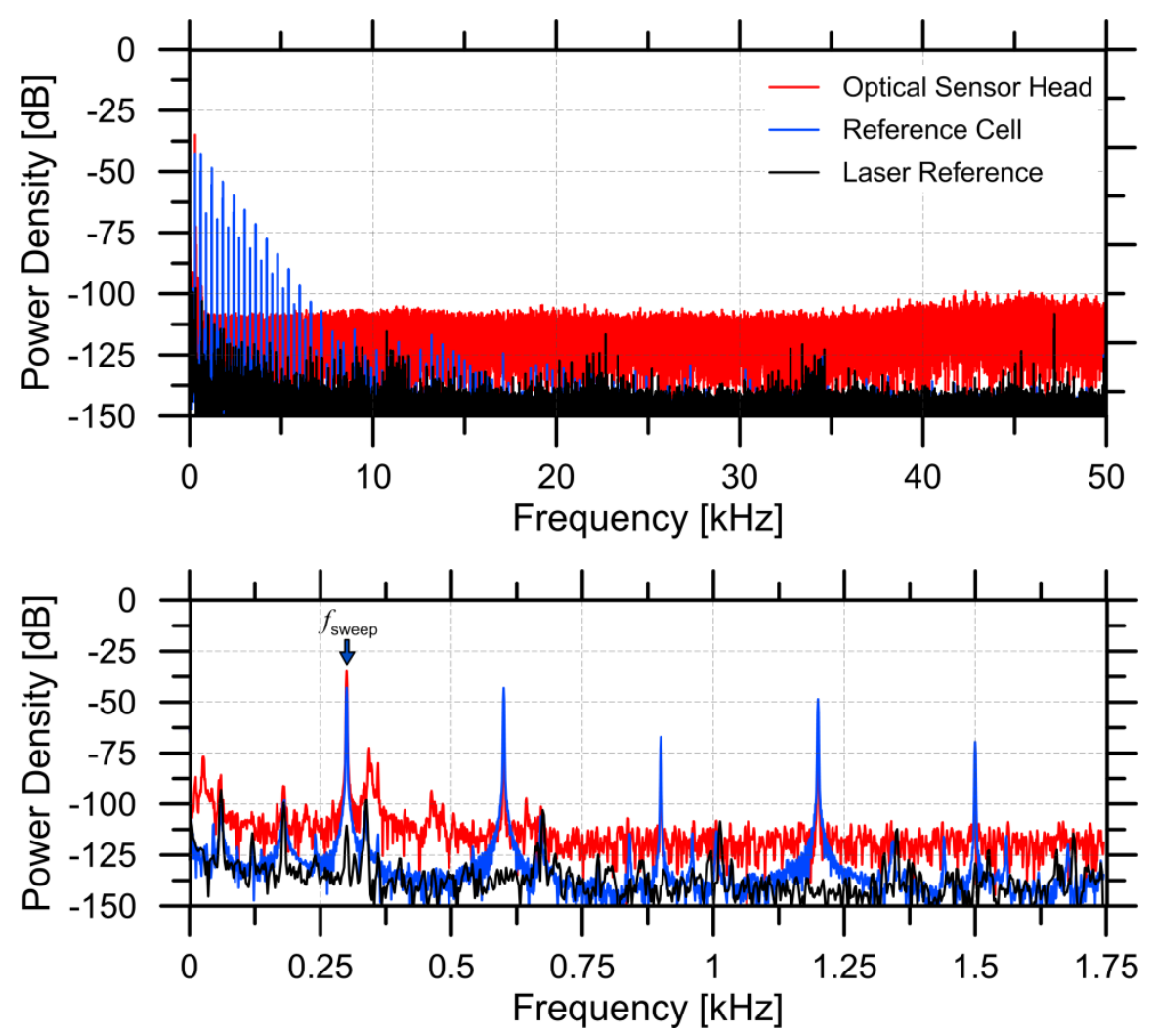

Figure 4.2: Fast Fourier transform for 10 seconds of SI laser reference, reference cell, and interrogation cell signals highlighting the measured SI signals at integer multiples of 300 Hz. Signals were acquired at a test chamber methane volume mixing ratio of $100 \mathbf{p p m}_{\mathrm{v}}$ at ambient temperatures and pressures using the $5.79 \mathrm{~m}$ multipass sensor head coupled to the

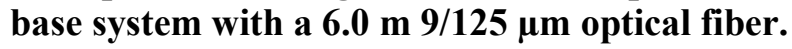

\footnotetext{
${ }^{3}$ Power spectrum FFT of 10 seconds of unfiltered experimental detector signals using a Gaussian window and exponential averaging
} 

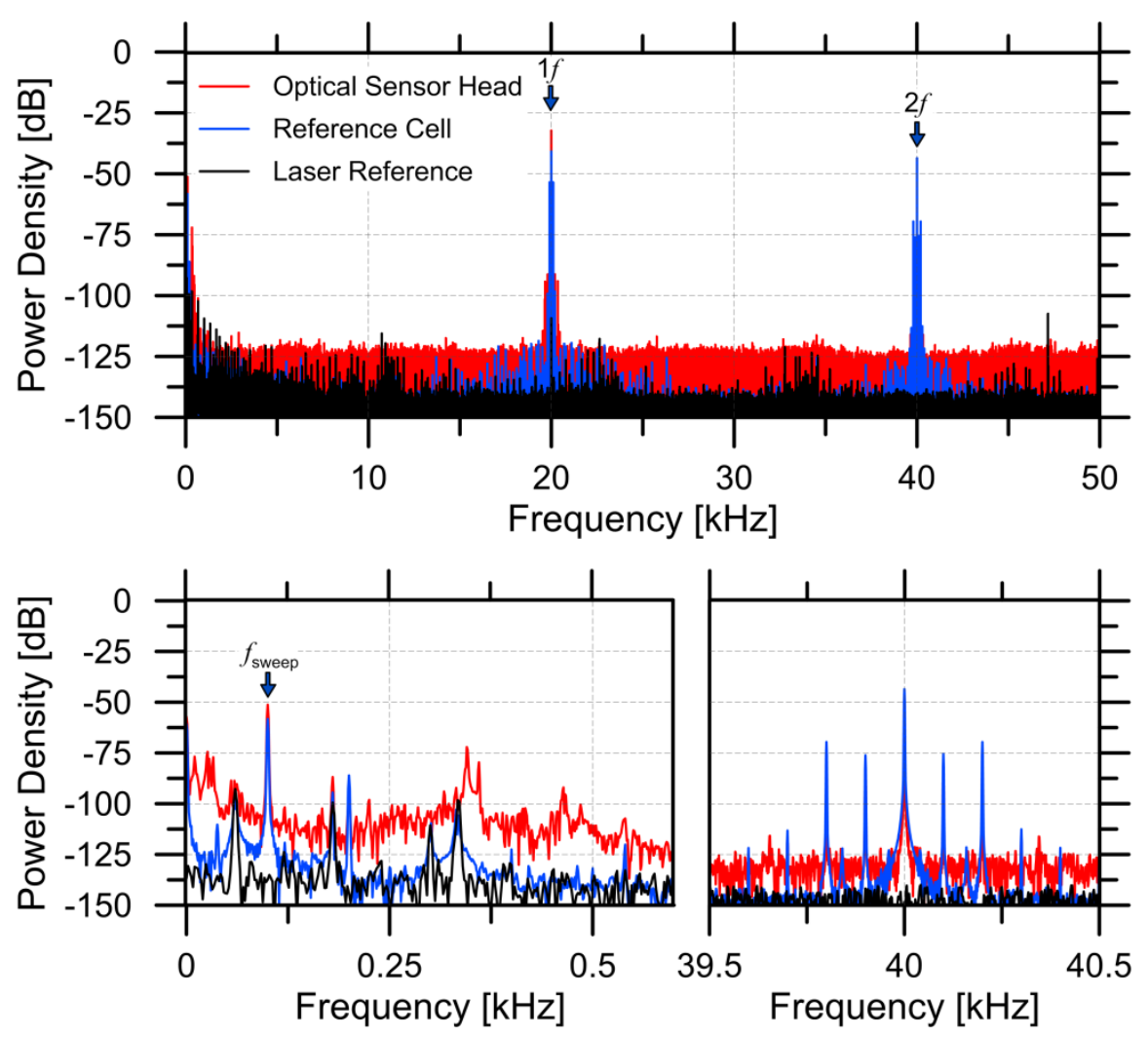

Figure 4.3: Fast Fourier transform for 10 seconds of WMS laser reference, reference cell, and interrogation cell signals highlighting the measured second-harmonic ( $2 f)$ WMS signals

for the combined modulation and sweep frequencies of $20 \mathrm{kHz}$ and $100 \mathrm{~Hz}$ respectively.

Signals were acquired at a test chamber methane volume mixing ratio of $100 \mathbf{p p m}_{\mathrm{v}}$ at ambient temperatures and pressures using the $5.79 \mathrm{~m}$ multipass sensor head coupled to the base system with a $6.0 \mathrm{~m} \mathrm{9/125} \mu \mathrm{m}$ optical fiber.

The threshold that separates periodic signals (wanted or unwanted) from the random white noise background is the known as the noise-floor. Generally the contribution of random white noise to the resultant signal increases as components are added between the signal generator and signal digitizer. The desired features (highlighted with arrows) in the return signals from the SI and WMS optical sensor head and reference cell have power densities greater than their respective noise floors. However, there are also unwanted periodic signals which have power spectral densities above the noise floor which may influence the quality of the experimentally derived volume mixing ratio. The most direct method of supressing unwanted signals and noise is at the source. Table 4.1 lists some possible unwanted signal sources and methods implemented to limit their influence. 
Table 4.1: Noise sources within the TDLAS system

\begin{tabular}{|c|c|c|}
\hline Source & Type & Mitigation Method \\
\hline Signal Generator(s) & $\begin{array}{l}\text { Unwanted Signals } \\
\text { White Noise }\end{array}$ & $\begin{array}{l}\text { Maximize Resolution } \\
\text { EM Shielding } \\
\text { Signal Generator Calibration }\end{array}$ \\
\hline Controller Current & $\begin{array}{l}\text { Drift } \\
\text { White Noise }\end{array}$ & $\begin{array}{l}\text { Sufficient Warm-up } \\
\text { Minimize Amplification }\end{array}$ \\
\hline Laser Diode & Drift & $\begin{array}{l}\text { Thermal Stabilization } \\
\text { Sufficient Warm-up }\end{array}$ \\
\hline Detector & White Noise & EM Shielding \\
\hline Power Source(s) & $\begin{array}{l}\text { Unwanted Signals } \\
\text { Line Signals } \\
\text { White Noise }\end{array}$ & $\begin{array}{l}\text { Source Filtering } \\
\text { Isolation Transformer } \\
\text { Low-noise AC-DC Supplies } \\
\text { Eliminated Grounding Loops }\end{array}$ \\
\hline EM Interference & $\begin{array}{l}\text { High-Frequency Signals } \\
\text { White Noise }\end{array}$ & $\begin{array}{l}\text { Shielded Cabling } \\
\text { Grounded Enclosures }\end{array}$ \\
\hline
\end{tabular}

If unwanted signal sources are known but unavoidable they may be suppressed with digital filtering. This can be an effective approach for supressing unwanted signals from mechanical vibrations and low-frequency power-source noise (Disselkamp et al., 2002; Cassidy and Reid, 1982a; Riris et al., 1994). To properly assess the TDLAS system performance, the presented results do not include any supplemental digital or analog filtering (beyond digital low-past filtering at the Nyquist frequency) unless explicitly stated.

The frequency difference between a constant, unwanted signal $\left(f_{\text {un }}\right)$, and desired signal (in this case the sweep frequency, $f_{\mathrm{s}}$ ) dictates the method required to supress the unwanted signal:

$\left\{f_{\text {un }}>f_{s}\right\}$ : averaging and/or filtering can be used (Hayward et al., 1989; Andersson et al., 2007; Riris et al., 1994).

$\left\{f_{\text {un }}=f_{s}\right\}$ : cannot be averaged out, but instead can be subtracted from the resultant signal with careful calibration (Chakraborty et al., 2010).

$\left\{f_{\text {un }}<f_{s}\right\}$ : manifests as a drift of the measured absorption- or $2 f$-peak height. It is possible to supress drift by signal averaging, but this requires datasets that capture multiple periods of the unwanted signal for sufficient suppression to minimize the 
risk of introducing bias errors (Rieker et al., 2009; Liu et al., 2004a). If $f_{\text {un }} \ll f_{s}$, real-time correction of signal drift may be possible.

\subsubsection{Background Signals}

Background signals are those which modify the desired signal with an identifiable temporal structure and a definite spectral power density at or near the frequency of the desired signal, $f_{\text {un }} \cong f_{s}$ (Kluczynski and Axner, 1999; Werle et al., 2004). These signals are undesirable since they may artificially alter the measured absorption leading to errors in the measured volume mixing ratio. If the sources of these signals are known and isolatable, they can be subtracted from the photodetector signals. Numerous authors have attempted this with varying degrees of success (Werle et al., 1993; Li et al., 2006; Hayward et al., 1989; Disselkamp et al., 2002). Residual amplitude modulation and optical interference fringe suppression using post-processing techniques have been demonstrated with reasonable success (Li et al., 2006; Chakraborty et al., 2010; Sun and Whittaker, 1992). The methods and outcomes of supressing background RAM and optical interference in the TDLAS system are discussed in Sections 2.2.2 and 5.3.2.

\subsubsection{Drift}

Drift results from low frequency signals, $f_{\text {un }}<f_{s}$, and adds bias errors to the calculated volume mixing ratio. Drift can be categorized as short, medium, and long term drift, corresponding to signals with periods on the order of seconds, hours, or days (or longer). The time scales and sources of these fluctuations dictate the relevant methods of compensation or suppression. Drift of laser wavelength and measured intensity are the biggest constraints on measurement accuracy. Methods of supressing or compensating for this wavelength and intensity drift are discussed in Chapter 5.

\subsection{Signal Pre-Processing}

Many SI and WMS signal processing techniques for deriving gas concentrations from absorption and harmonic signals have been documented (Lackner, 2007; Werle, 1998; Shemshad et al., 2012; Song and Jung, 2003). The general method used in the present 
system does not follow any one method in particular, but instead has been refined and adapted from examples found in literature to achieve the accuracy and sample-rates required for this particular application. The SI absorption or second-harmonic (2f) WMS post-absorption signals are generated using the raw photodetector signals (obtained by modulating the laser driving current over the $2 v_{3} R(3)$ methane manifold) and the estimated absorption-free intensity. These signals are then digitally processed to yield absorption or $2 f$ values, which are subsequently converted to a methane volume mixing ratio (VMR) estimate.

\subsubsection{Absorption-Free Intensity}

An estimate of the absorption-free intensity, $I_{0}{ }^{*}$, is required to convert the measured postabsorption intensities for the reference cell and sensing head to absorption and $2 f$ signals following (Eq. 2.1):

$$
I_{0}^{*} \approx I_{0}=\frac{I}{\tau}
$$

Many systems purge interrogation volumes with a 'zero-gas' (a gas containing either zero or a known VMR of the absorbing species of interest) to estimate the absorption-free intensities (Disselkamp et al., 2002; Wienhold et al., 1994; Uehara and Tai, 1992). This technique would not be practical for this system where fiber-coupled sensing heads are installed throughout an industrial facility. Three alternate methods of estimating the absorption-free intensity are presented and discussed.

\subsubsection{Pre-run Calibration}

Long-term laser degradation has a significant impact on the consistency of the intensity and wavelength output from laser diodes (Johnson, 2006; Linnerud et al., 1998). This permanent drift is most severe in the first few days of diode operation, often called the wear-in period. The diodes used in the TDLAS system were worn-in before they were characterized and packaged at the manufacturing facility. In this present system, a prerun calibration is performed to estimate laser characteristics and monitor changes in laser performance that may be relevant to methane VMR estimates. The calibration is 
conducted by driving the injection current of the laser diode using the optimum modulation and sweep signals at off-peak wavelengths on either side of the near-IR $2 v_{3}$ $\mathrm{R}(3)$ methane absorption manifold, and observing the return intensity for the laser reference, reference cell, and sensing head over a 30 second period. The laser wavelength is adjusted by thermally tuning the laser diode to temperatures above and below, $T_{c}\left[{ }^{\circ} \mathrm{C}\right]$, the temperature which tunes the output laser wavelength to the center of the $\mathrm{R}(3)$ manifold, $v_{p}$. The laser intensity characteristics at $T_{c}$ are then linearly interpolated from the measured absorption-free values to obtain estimates of the absorption-free mean intensity $\left(\bar{I}_{0}^{*}\right)$, and the first- and second-order intensities $\left(i_{0}^{*}\right)$ and $\left(i_{2}{ }^{*}\right)$, as demonstrated in Figure 4.4 using a typical pre-run calibration set. Similarly, the phase difference between the intensity and wavelength can be estimated for all channels, $\left(\psi_{1}^{*}\right)$ and $\left(\psi_{2}^{*}\right)$.

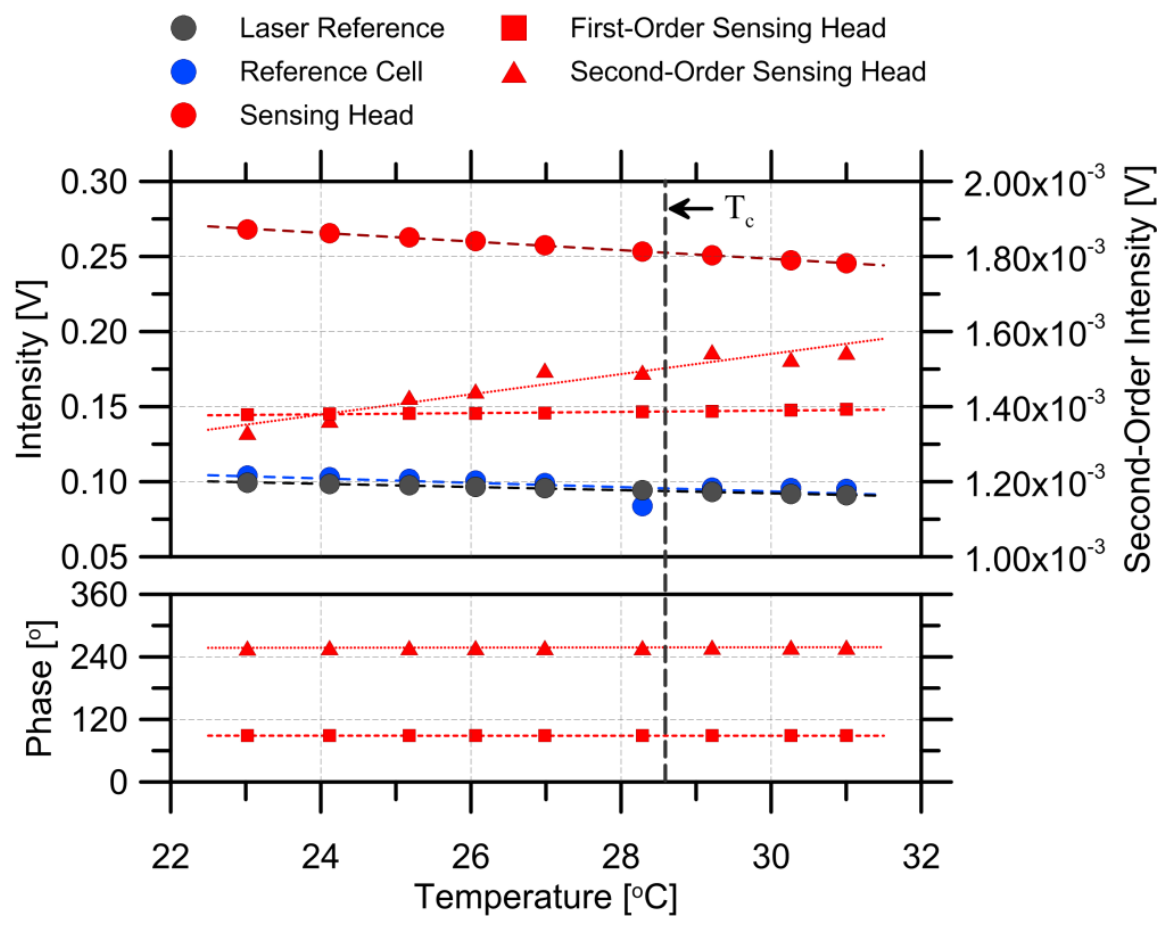

Figure 4.4: Linear estimates of the absorption-free temperature dependant laser characteristics responding to an input-current sweep signal of $100 \mathrm{~Hz}$ with an amplitude of $0.075 \mathrm{~V}(\sim 1.5 \mathrm{~mA})$. Mean sweep intensity is denoted using circles, the first- and secondorder harmonic characteristics (intensity amplitude and wavelength/intensity phase-shift) are denoted by squares and triangles respectively. 
As the laser characteristics changed with time and use, the mean laser driving current (also known as the laser current offset), $\bar{A}[\mathrm{~mA}]$, required to center the laser diode output wavelength on the $2 v_{3} \mathrm{R}(3)$ methane absorption manifold, changed. This resulted in a fluctuation of the observed laser characteristics as illustrated in Figure 4.5, acquired at ambient pressures, temperatures, and methane concentrations, with an optical path length of $5.79 \mathrm{~m}$.

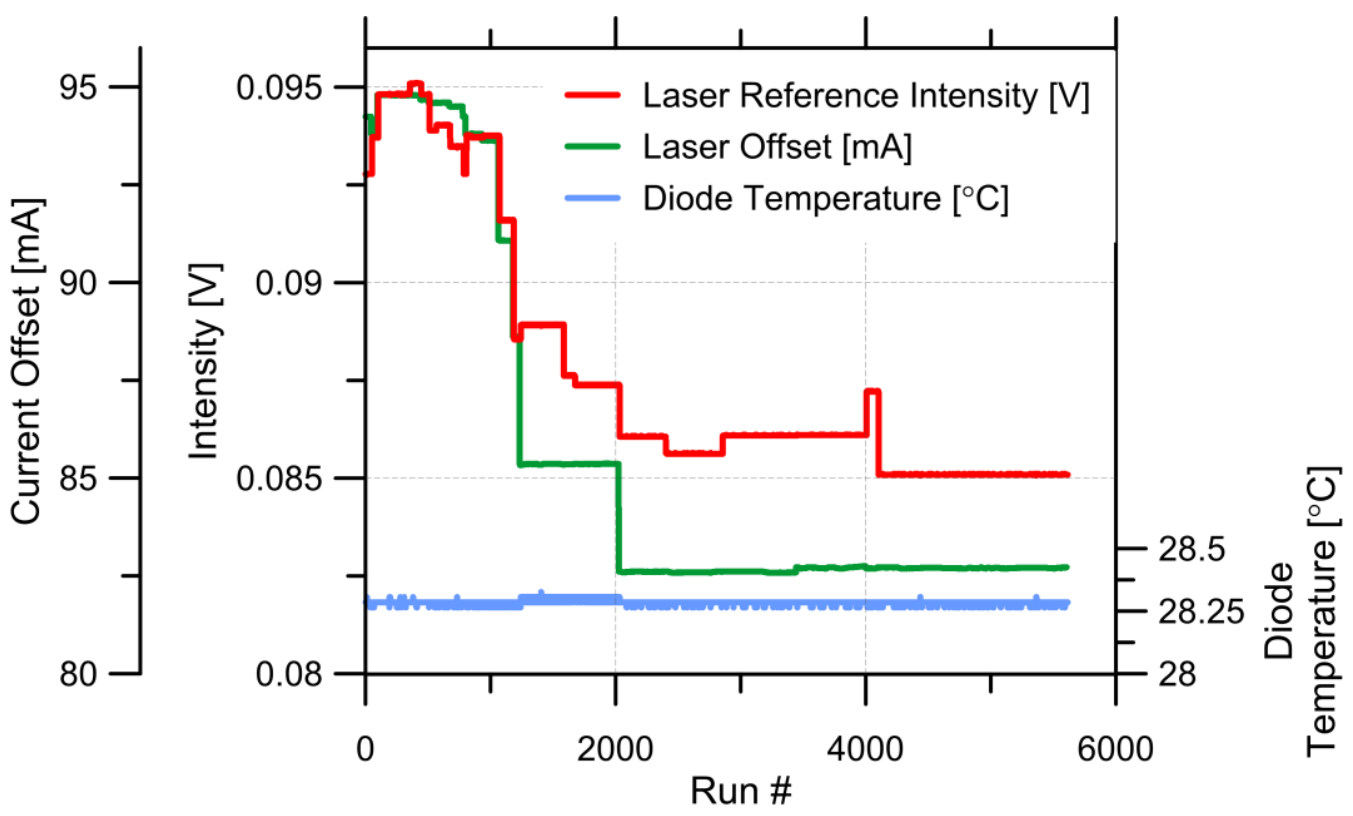

Figure 4.5: Laser output drift over 5700 separate data-acquisition 'runs'. Plotted is the resultant mean intensity for the combined diode temperature and injection current offset to stay centered on the $2 v_{3} R(3)$ methane manifold. Data acquired at ambient pressures, temperatures, and methane concentrations, with an optical path length of $5.79 \mathrm{~m}$.

To account for changing laser characteristics, the above laser characterization ('prerun calibration') was performed daily, with the following generalizations:

- Laser intensity and wavelength are linear over the tuning range

- Laser diode temperature and subsequent wavelength is stable

- Laser intensity response stays constant over calibration process $(\sim 20$ minutes)

The above absorption-free characteristics are applicable when the TDLAS system is operated in steady laboratory conditions. However, even under these conditions it has been observed that the laser intensity fluctuates from the obtained absorption-free values 
over the course of a day, limiting the interval over which the pre-run calibration is accurate. For improved accuracy over the course of a day, additional methods of obtaining accurate absorption free intensity are required, discussed in Sections 4.3.1.2 and 4.3.1.3.

\subsubsection{Concurrent Intensity Correction}

In an attempt to compensate for drifting return intensity, the mean post-absorption optical sensing head signals ( $\bar{I}_{S}$ and $\left.\bar{I}_{\text {WMS }}\right)$ were used as an estimate of the mean absorption-free

intensities $\left(\bar{I}_{0_{S}}{ }^{*}\right.$ and $\left.\bar{I}_{0 \mathrm{WMS}}{ }^{*}\right)$ over the time-averaging interval. The time-averaging interval includes an integer number of sweeps, $N$, with period $t_{s}=1 / f_{s}$, such that the time-averaging interval is $N t_{s}[\mathrm{~s}]$. The estimated mean post-absorption intensity for the SI and WMS methods can be written respectively as:

$$
{\overline{I_{0}}}^{*} \approx \bar{I}_{s}=\frac{1}{Z} \sum_{i=0}^{Z} I_{s}\left(t_{i}\right)
$$

and

$$
{\overline{I_{0}}}_{W M S}{ }^{*} \approx \bar{I}_{W M S}=\frac{1}{Z} \sum_{i=0}^{Z} I_{\mathrm{WMS}}\left(t_{i}\right)
$$

where $Z$ is total number of samples in a time-averaging interval ( $Z=N \times$ (sample frequency) $\left./ t_{s}\right)$ and $t_{i}$ is the sample index. Since the concurrent intensity correction is derived directly from the post-absorption sensing head signals, it inherently compensates for some degree of intensity drift. However, due to the influence of absorption on the post-absorption signals, the concurrent intensity correction has the disadvantage of underestimating the absorption-free intensity, where magnitude of the underestimate increases as the methane volume mixing ratio within the sensing head increases. Chapter 5 discusses the influence of this on the measurement performance of this TDLAS system for various system configurations. 


\subsubsection{Reference Laser}

A third means of estimating the optical sensing head absorption free intensity is possible by monitoring the absorption-free intensity at a nearby off-manifold wavelength targeted with a second laser. This technique is implemented in this system with a $2 \times 2$ optical relay (see Section 3.1.3) to quickly switch between absorption (L2) and reference (L1) laser diodes. The reference laser is tuned to a spectral region near the $2 v_{3} \mathrm{R}(3)$ methane manifold (centered at approximately $1664.24 \mathrm{~nm}$ or $6008.75 \mathrm{~cm}^{-1}$ ) where there is negligible attenuation by methane or other atmospheric gases. Figure 4.6 illustrates the close correlation between the post-absorption return intensities of the reference and absorption lasers measured from the sensing head, where the plotted data were collected sporadically over a period greater than one year.

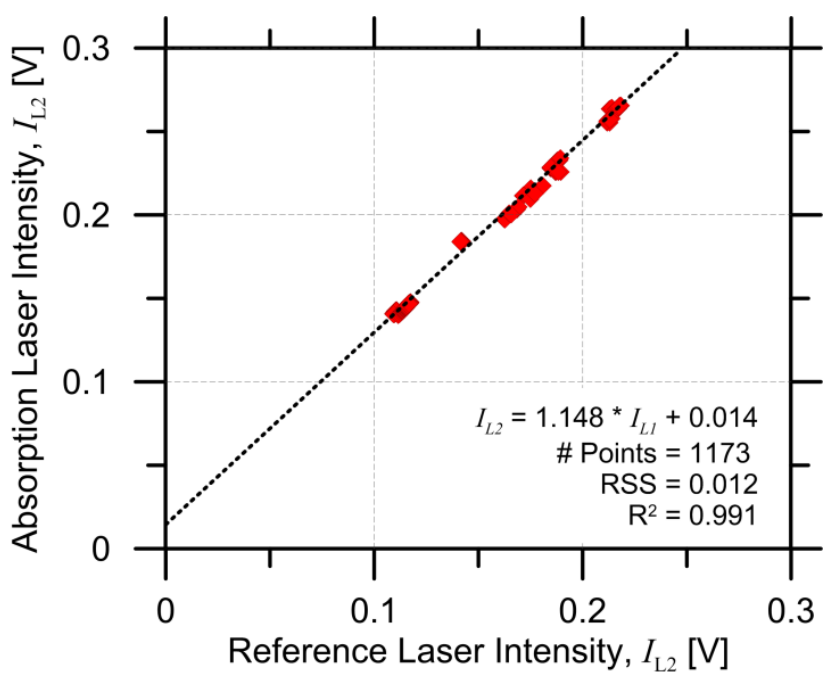

Figure 4.6: Relationship between reference (L1) and absorption (L2) mean laser intensities using the basic system configuration, at ambient temperature and pressure, and at ambient methane volume mixing ratios

To ensure the most accurate results, the pre-run absorption-free interrogation cell mean intensity estimates for L1 and L2, $\bar{I}_{0}{ }_{\mathrm{L} 1}$ and $\bar{I}_{0}{ }_{\mathrm{L} 2}$, and the intercept, $\widehat{\bar{I}}_{\mathrm{L} 2}(0)$, are used to calculate the relationship between the absorption and reference laser intensities, $\hat{\phi}$, following: 


$$
\hat{\phi}=\frac{{\overline{I_{0}}}_{\mathrm{L} 2}^{*}-{\widehat{\overline{I_{0}}}}_{0_{\mathrm{L} 2}}(0)}{{\overline{I_{0}}}_{\mathrm{L} 1}^{*}}=\frac{\bar{I}_{0_{\mathrm{L} 2}}-{\widehat{\overline{I_{0}}}}_{\mathrm{L} 2}(0)}{\bar{I}_{0 \mathrm{~L} 1}}
$$

where $\bar{I}_{0 \mathrm{~L} 2}$ and $\bar{I}_{0 \mathrm{~L} 1}$ are the concurrently measured mean intensities (over one sweep period) for the absorption and reference lasers respectively. Using $\hat{\phi}$, the SI and WMS absorption-free absorption laser intensity signal, $I_{0}{ }_{\mathrm{L} 2}(t)$, can be estimated using the mean reference laser intensity, $\bar{I}_{0 \mathrm{~L} 1}$, and Equations 2.15 and 2.21 following:

$$
\begin{aligned}
& I_{0}{ }_{\mathrm{L} 2, \mathrm{SI}}(t)=\left(\bar{I}_{0 \mathrm{~L} 1} \hat{\phi}+\widehat{\bar{I}}_{\mathrm{L} 22}(0)\right) \\
& {\left[1+i_{0}{ }^{*}{ }_{\mathrm{s}} \cos \left(2 \pi f_{s} t+\psi_{2}{ }^{*}{ }_{\mathrm{s}}\right)+i_{2}{ }^{*}{ }_{\mathrm{s}} \cos \left(4 \pi f_{s} t+\psi_{2}{ }^{*}{ }_{\mathrm{s}}\right)\right]} \\
& I_{0}{ }_{\mathrm{L} 2, \mathrm{WMS}}(t)=\left(\bar{I}_{0 \mathrm{~L} 1}, \hat{\phi}+\widehat{\bar{I}}_{0 \mathrm{~L} 2}(0)\right) \\
& {\left[1+i_{0}{ }^{*} \cos \left(2 \pi f_{s} t+\psi_{2}{ }^{*} \mathrm{~s}\right)+i_{2}{ }^{*}{ }_{\mathrm{s}} \cos \left(4 \pi f_{s} t+\psi_{2}{ }^{*}{ }_{\mathrm{s}}\right)\right.} \\
& \left.+i_{0}{ }^{*}{ }_{\mathrm{m}} \cos \left(2 \pi f_{m} t+\psi_{2}{ }^{*}{ }_{\mathrm{m}}\right)+i_{2}{ }^{*}{ }_{\mathrm{m}} \cos \left(4 \pi f_{m} t+\psi_{2}{ }^{*}{ }_{\mathrm{m}}\right)\right]
\end{aligned}
$$

Estimating the absorption-free laser intensity using a second laser to improve measurement performance for the SI and WMS techniques and system configurations is demonstrated in Section 5.3.

\subsection{Acquisition Procedure}

Figure 4.7 illustrates the timing of operations making up a single run (to acquire up to 5 seconds of continuous data) for the SI or WMS methods. The longest portion of each run was the 6-minute time required to ensure the test chamber methane VMR settled. Next was the measurement of atmospheric (laboratory) conditions and mass-flow controller (MFC) readings, requiring approximately $15 \mathrm{~s}$. Subsequently the system obtained reference signals using the reference laser, requiring a total of $1.2 \mathrm{~s}$. The cycle was concluded by switching back to the absorption laser and obtaining up to $5.0 \mathrm{~s}$ (generally $1.05 \mathrm{~s}$ ) of simultaneous data from the sensing head and reference cell. In a field measurement setting, the long delay to ensure the methane VMR settled would not be required and the system could be set up (with minimal re-programming) to measure 
methane at many multiplexed sensing heads achieving a $1.0 \mathrm{~s}$ measurement at a different sensing head every $2.2 \mathrm{~s}$.

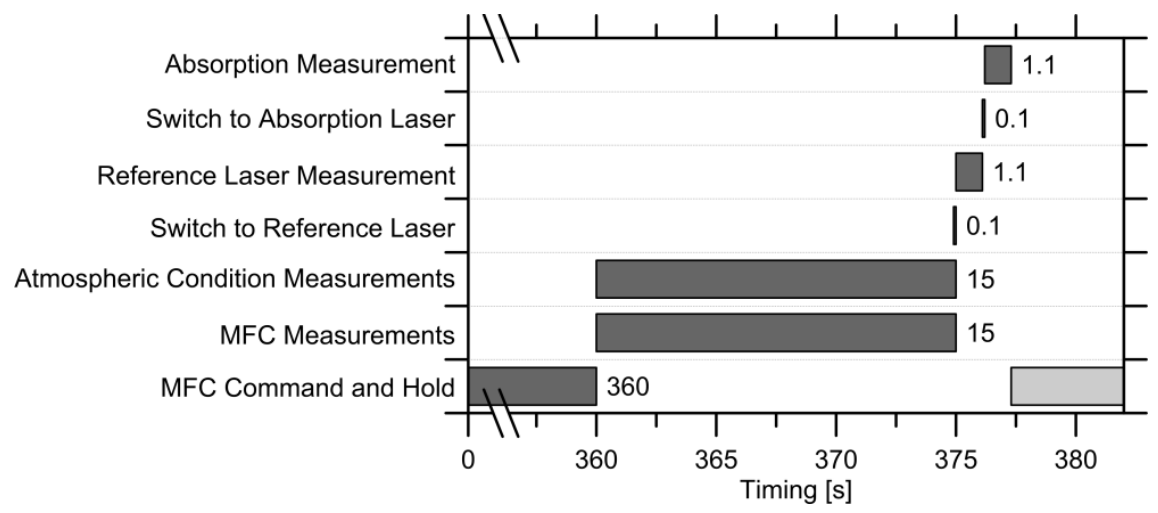

Figure 4.7: The procedure to acquire a calibration data point ('run')

The digitized detector signals were transferred to the random access memory on the PXI chassis controller, from where it was compressed and saved to the hard drive as a binary file. A single binary file contained up to one minute of digitized signals from multiple simultaneously-sampled photodetectors. Each file contained raw digitizer signal data and other pertinent information about the dataset such as experiment type, control settings, MFC rates, and pre- and post-run laboratory conditions.

The system was configured to observe the methane VMR in real-time to aid in system trouble shooting and post-processing refinement. Figure 4.8 illustrates these methane measurements overlaid on the requested test chamber methane VMR (black dash) and the $1 \mathrm{~s}$ averaged results (red dot) and associated measurement statistics. These results were obtained at atmospheric temperature and pressure using the $5.79 \mathrm{~m}$ sensor head. 


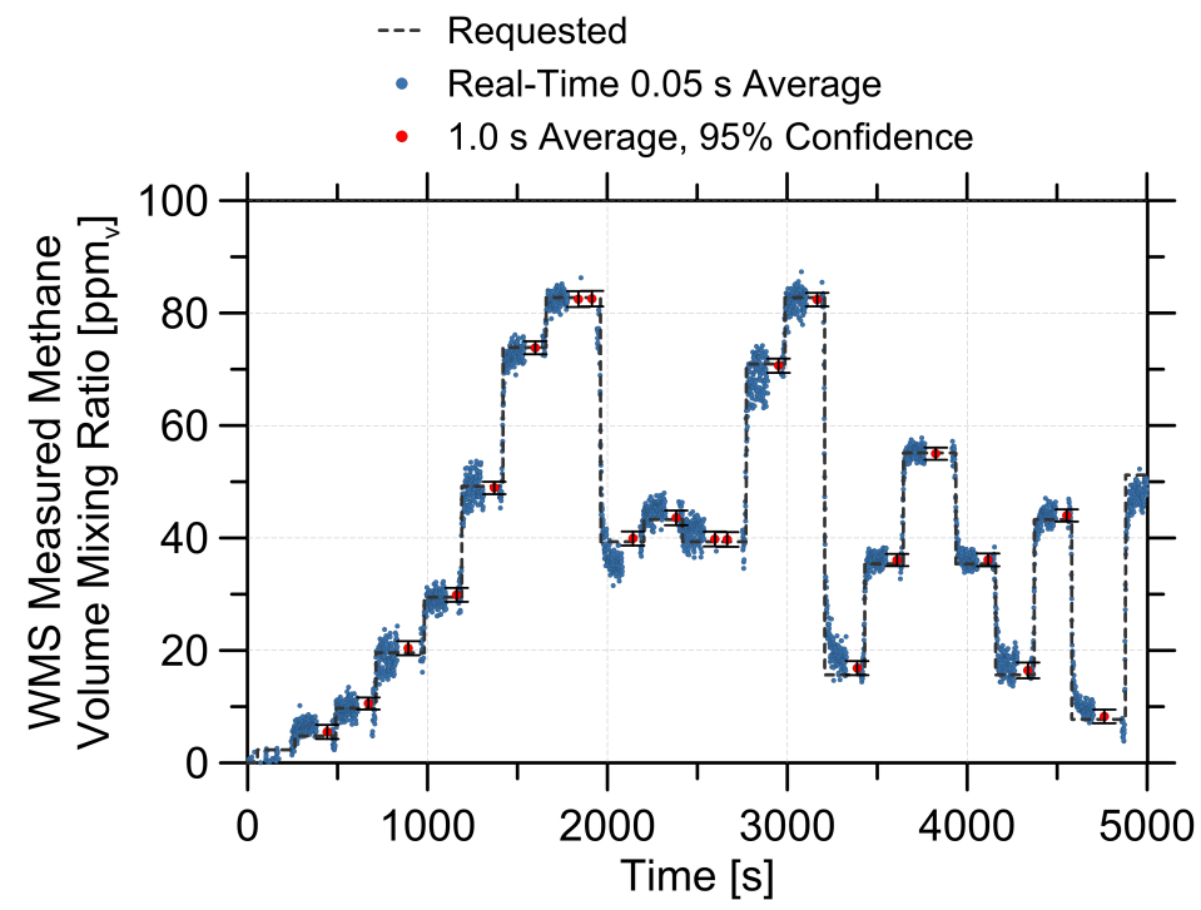

Figure 4.8: 'Real-time' WMS-based measurements (blue) overlaid on the requested test chamber methane volume mixing ratio measurement results (red) including $95 \%$ uncertainties

\subsection{Signal Processing}

Photodetector signal digitization and subsequent software based post-processing was selected over analog methods for ease of system expansion and modification, and to permit refinement of signal processing techniques with minimal downtime. Each 'run' dataset was processed using various techniques and algorithms to determine the processing scheme that yielded the most desirable measurement precision and lower detection limit, the results of which are presented in Chapter 5.

During each sinusoidal sweep period, the absorption or $2 f$ feature is traversed twice, once as the wavelength is increasing, and once as the wavelength is decreasing. Features measured as the wavelength is increasing or decreasing are referred to as the leading or lagging features respectively. The influence of using either or both of these features in subsequent calculations is discussed further in Section 5.3.2. It can be presumed that the leading feature is used exclusively, unless otherwise noted. 


\section{Sweep Integration Signal Processing}

The absorption signal, $R_{\mathrm{abs}}(t)$, for the SI method was calculated following Equation 2.18 (i.e. the ratio of the measured signal after absorption and the absorption free intensity estimate). Figure 4.9 illustrates the basic signal processing steps which transform the measured signals from the sensing head and reference-cell to time-resolved absorption peak heights and subsequently a methane volume mixing ratio.

\section{Wavelength Modulation Signal Processing}

Similar to the SI technique, the WMS technique relies on an estimate of the absorptionfree intensity and a wavelength reference, $v_{p}$, indicating the wavelength of the $2 f$-feature maximum within the WMS signal, $R_{2 f}(t)$, to enable a volume mixing ratio measurement. Figure 4.10 illustrates the basic post-processing steps to convert the measured multipass and reference-cell signals to $2 f$-peaks, $x_{2 f}$, and subsequently to a methane volume mixing ratio. 


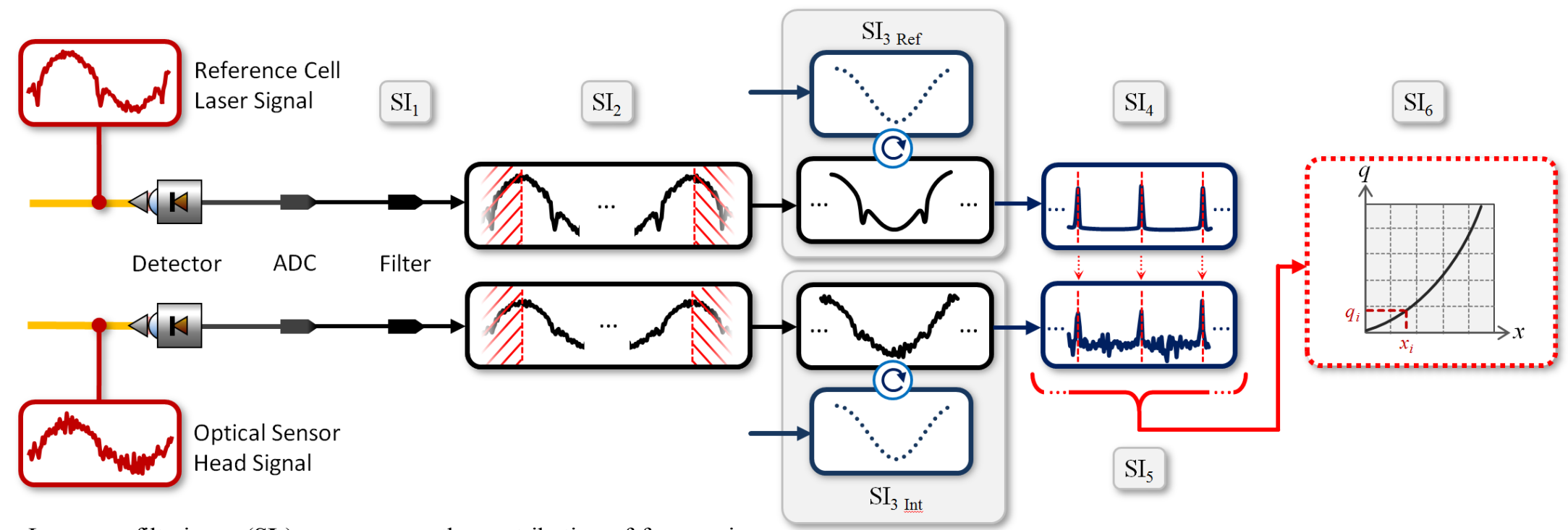

Low-pass filtering - $\left(\mathbf{S I}_{\mathbf{1}}\right)$ - suppresses the contribution of frequencies which cannot be fully resolved by the digitizer at the sample rate used ( $\left.f_{\text {samp }}\right), f_{\text {filter }}=0.25 * f_{\text {samp. }}$.

Signal pre-processing - $\left(\mathbf{S I}_{\mathbf{2}}\right)$ - removes phase-delay between the reference cell and multipass cell signals and removes any incomplete sweep periods.

Reference cell absorption signal - $\left(\mathbf{S I}_{\mathbf{3}}\right.$ Ref $)$ - estimate the reference cell absorption signal: the quotient of the estimated absorption-free reference cell signal (from the pre-run calibration data) with the post-absorption reference cell signal

Sensing head absorption signal - ( SI $_{3}$ Int $)$ - combines the estimated absorption-free interrogation cell signal with the post-absorption interrogation cell signal to estimate the interrogation cell absorption signal.

Reference cell absorption peaks - $\left(\mathbf{S I}_{\mathbf{4}}\right)$ - determines the reference cell absorption peak values and sample indices.

Absorption peaks - $\left(\mathbf{S I}_{\mathbf{5}}\right)$ - utilizes the reference cell absorption peak time indices to obtain the interrogation cell absorption values.

Volume mixing ratio - $\left(\mathbf{S I}_{\mathbf{6}}\right)$ - provides an estimate the volume mixing ratio within the interrogation cell using various methods discussed in Section 4.6

Figure 4.9: Sweep integration signal processing schematic 


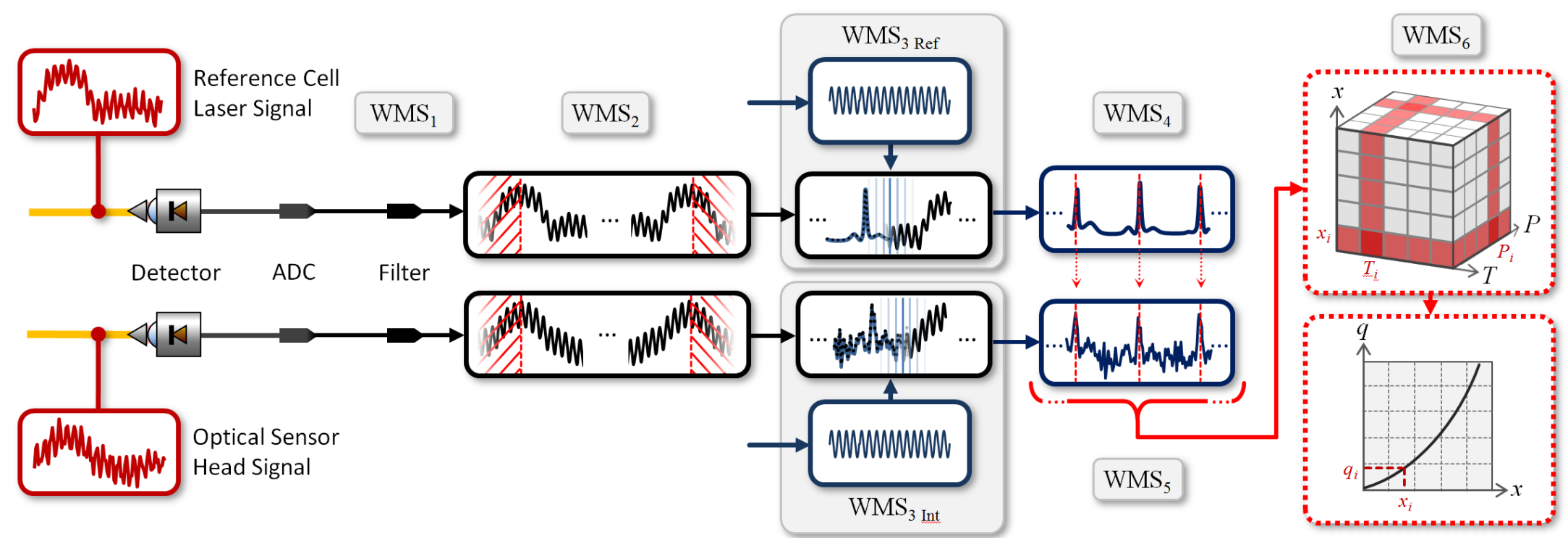

Low-pass filtering - $\left(\mathbf{W M S}_{1}\right)$ - suppresses the contribution of frequencies which cannot be fully resolved by the digitizer at the sample rate used $\left(f_{\text {samp }}\right), \mathrm{f}_{\text {filter }}=0.25 * \mathrm{f}_{\text {samp }}$.

Signal pre-processing - $\left(\mathbf{W} \mathbf{M S}_{\mathbf{2}}\right)$ - removes phase-delay between the reference cell and multipass cell signals, and removes any incomplete sweep periods.

Reference cell $2 f$ signal - $\left(\mathbf{W M S}_{3 \text { Ref }}\right)$ - converts the reference cell absorption signal in to a second harmonic signal using the demodulation technique outlined in Section 2.2.

Sensing head $2 f$-Peaks $-\left(\mathbf{W M S}_{3}\right.$ Int $)$ - converts the interrogation cell signal in to a $2 f$ signal using the demodulation technique outlined in Section 2.2.

Reference cell $2 f$-Peaks - $\left(\mathbf{W M S}_{4}\right)$ - determines the reference cell $2 f$ peak values and sample indices.

Interrogation cell $2 f$-peaks - $\left(\mathbf{W M S}_{\mathbf{5}}\right)$ - utilize the reference cell $2 f$-peak sample indices to obtain the interrogation cell $2 f$-peak values.

Volume Mixing Ratio Measurement - $\left(\mathbf{W M S}_{6}\right)$ - provides an estimate the volume mixing ratio within the interrogation cell using various methods discussed in Section 4.6 and measured atmospheric pressure and temperature.

Figure 4.10: Wavelength modulation spectroscopy signal processing schematic 


\subsection{Volume Mixing Ratio Measurement}

The volume mixing ratio can be derived from laser absorption and harmonic signals using many methods that can be roughly categorized as either using theory-based estimations of the measureable concentrations in conjunction with a secondary empirical calibration (termed a "theory-based calibration") or using an exclusively experimental calibration (termed an "experimental calibration"). Both methods require comparison to a well-known source. In the present experiments, this was accomplished by generating known methane volume mixing ratios in the test chamber (see Appendix A).

For the theory-based calibration method, the methane volume mixing ratio (VMR) was derived from the absorption and $2 f$ harmonic signals using pre-computed look-up table to permit real-time VMR measurement with low computational effort. The look-up tables were theory-based approximations of SI absorption (Equations 2.17 and 2.18) and WMS $2 f$ (Equations 2.27, 2.28, and 2.31) values calculated over a range of methane volume mixing ratios and atmospheric conditions representative of the conditions in which the sensing head will operate. By contrast, the experimental calibration approach used an experimentally determined calibration curve to calculate methane volume mixing ratio directly from measured absorption or $2 f$ values, $x_{\mathrm{abs}}$ or $x_{2 f}$, and the estimated absorption-free intensity from the remote optical sensor.

Further details and examples of theory- and experimental-based calibration techniques for the SI and WMS methods are provided in Appendix B. Analysis of system performance using these different calibration approaches are discussed in Chapter 5 for various system configurations and post-processing methods. Appendix C outlines the procedure for calculating measurement precision and lower detection limit. 


\section{Chapter 5 Results}

This chapter presents the results from a series of systematic experiments that quantitatively evaluated system hardware and signal processing methods based on their ability to minimize system drift, and lower detection limit (LDL) and measurement precision values. The best-performing WMS and SI-based system configurations were selected and evaluated against the target methane volume mixing ratio measurement precision and LDL required for quantifying fugitive methane plumes using statistical trajectory methods (Brereton and Johnson, 2012) and the published performance of similar fiber-based measurement systems.

\subsection{Optimum Laser Driving Signal}

The optimum laser driving signal frequencies and amplitudes are often assumed to occur when the $2 f$ signal is maximized for the WMS technique (Chambers et al., 2004; Liu et al., 2004b; Schilt et al., 2003; Rieker et al., 2009), and when the absorption signal is maximized for the SI technique. For the present system, optimal driving signal parameters for both the SI and WMS techniques were determined considering:

1. Signal-to-noise ratio: $\mathrm{SNR}=\bar{x} / s_{x}$, where $\bar{x}$ and $s_{x}$ are the mean and standard deviation of experimentally measured absorption or $2 f$ values averaged for one second,

2. Sensitivity: $G^{\prime}=\Delta x / \Delta q$, the change in measured absorption or $2 f$ value for a given change in test chamber methane volume mixing ratio (VMR), and

3. Absolute difference between the theory-based and measured absorption or $2 f$ values: $(1-|(x-R) / x|)$.

A study was conducted to determine the optimum laser driving signals within the limits of the laser controllers and data acquisition system. As illustrated in Figure 5.1, the maximum sweep and modulation signal amplitudes were constrained to $1.0 \mathrm{~V}$. This 
allowed the signal generators to operate at a lower gain setting, ensuring full 16-bit resolution over their $\pm 1 \mathrm{~V}$ output range. The minimum sweep and modulation amplitudes were constrained by the $\sim 0.075 \mathrm{~V}$ amplitude minimum of the signal generators. The minimum possible sweep frequency of $\sim 40 \mathrm{~Hz}$ at low amplitudes and $\sim 80 \mathrm{~Hz}$ at high amplitudes was constrained by the capability of the signal generator. The maximum possible sweep frequency of $\sim 2.5 \mathrm{kHz}$ was constrained by the bias tee. The maximum $(100 \mathrm{kHz})$ and minimum $(10 \mathrm{kHz})$ laser modulation frequencies were constrained by the data acquisition hardware and laser controller stability respectively.

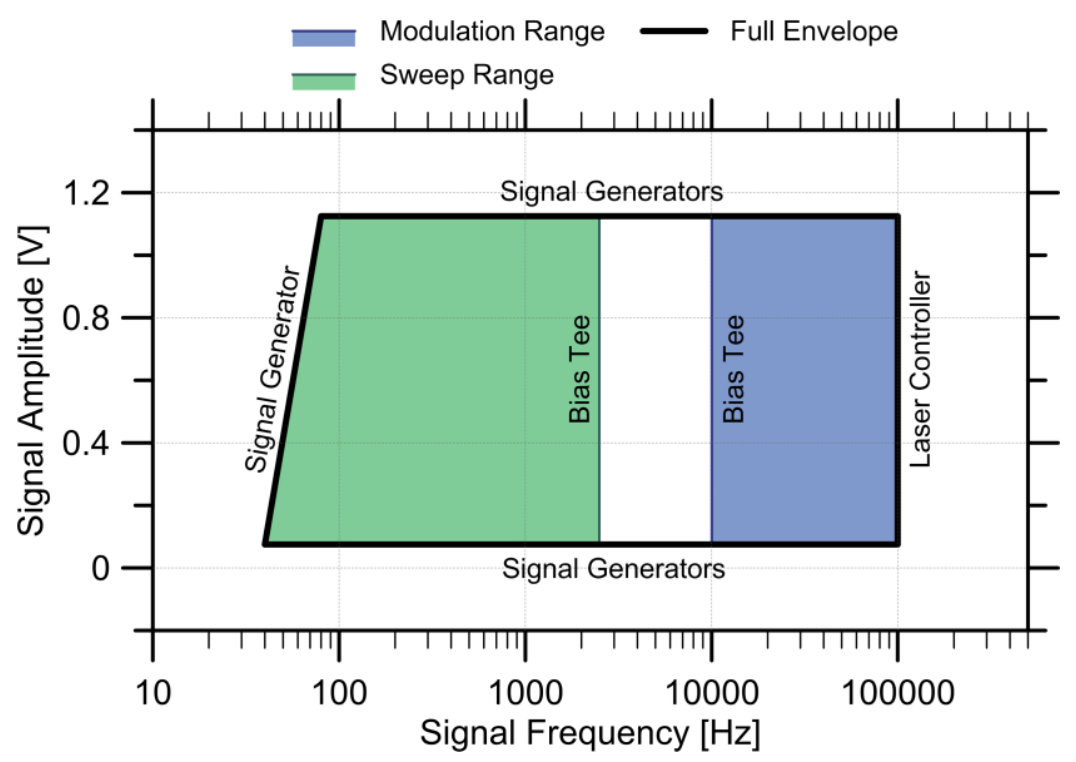

Figure 5.1: Laser driving signal amplitude and frequency operation envelope as determined by hardware specification data

To limit computational and experimental time, the search space for the optimum driving signal did not include WMS modulation amplitudes below $0.5 \mathrm{~V}$ and sweep amplitudes above $0.2 \mathrm{~V}$, as these consistently yielded inferior results. Table 5.1 lists the ranges within which the optimum driving signals for the SI and WMS techniques were determined. 
Table 5.1: Laser driving signal characterization ranges

\begin{tabular}{c|c|c|c|c|c}
\hline \multirow{2}{*}{ Method } & \multicolumn{4}{c}{ Signal Parameter } \\
\cline { 3 - 6 } & & \multicolumn{2}{c}{ Amplitude [V] } & \multicolumn{2}{c}{ Frequency [Hz] } \\
& & Min. & Max. & Min. & Max. \\
\hline SI & Sweep & 0.075 & 1.125 & 80 & 2,500 \\
\hline \multirow{2}{*}{ WMS } & Sweep & 0.075 & $0.2 \dagger$ & 80 & 400 \\
& Modulation & 0.5 & $1.125 \dagger$ & 10,000 & 100,000 \\
\hline
\end{tabular}

The optimum driving signals were determined from experiments using the $5.79 \mathrm{~m}$ fiber-coupled optical sensor head coupled via 1.0 meter optical fiber to the thermally stabilized enclosure. Measurements were made with test chamber methane volume mixing ratios between 2.5 and $100 \mathrm{ppm}_{\mathrm{v}}$, at standard lab temperature and pressure. The methane $2 v_{3} \mathrm{R}(3)$ absorption and second-harmonic signals were calculated using the absorption-free intensity signal estimated from the concurrent intensity correction scheme (see Section 4.3.1) at the manifold center estimated using the reference cell signal maximum (see Section 5.3.1).

\subsubsection{Optimum Laser Driving Sweep Frequency and Amplitude for SI Method}

The influence of laser sweep frequency and amplitude on absorption signal SNR, measurement sensitivity, and correspondence to theory for the SI method are illustrated in Figure 5.2. Plotted data are divided by their respective maximum values. The sample data shown are for experiments with a methane VMR of $10 \mathrm{ppm}_{\mathrm{v}}$, and are representative of the trends observed between 2.5 and $50 \mathrm{ppm}_{\mathrm{v}}$. 

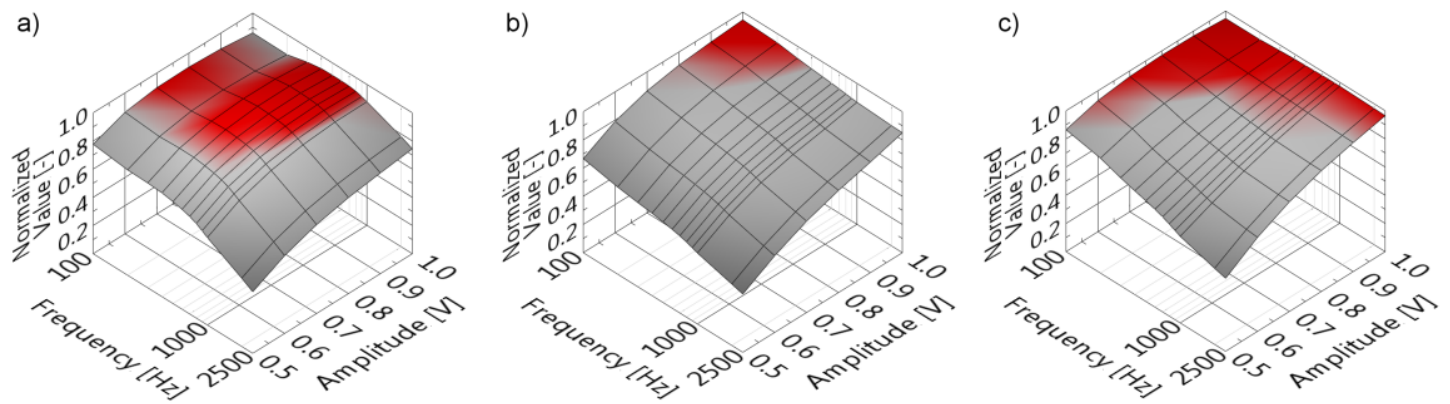

Figure 5.2: Surfaces indicating the normalized values of a) SNR, b) sensitivity, and c) the difference between the theory-based and measured absorption values using the SI technique. Data was obtained using the $2 v_{3} R(3)$ methane manifold for a methane at a volume mixing ratio of $10 \mathrm{ppm}_{\mathrm{v}}$ in nitrogen, at standard atmospheric conditions. High contour values (red) indicate regions of desirable signal attributes.

As shown in Figure 5.2, the maximum absorption value SNR occurs at moderate sweep frequencies and high modulation amplitudes, whereas the sensitivity and experimentally measured absorption matches theory-based estimations most closely at high sweep amplitudes and sweep frequencies. Figure 5.3 illustrates the equallyweighted sum of the normalized parameters, which yields an optimum (denoted by the red region) sweep frequency and amplitude at approximately $300 \mathrm{~Hz}$ and $1.0 \mathrm{~V}$.

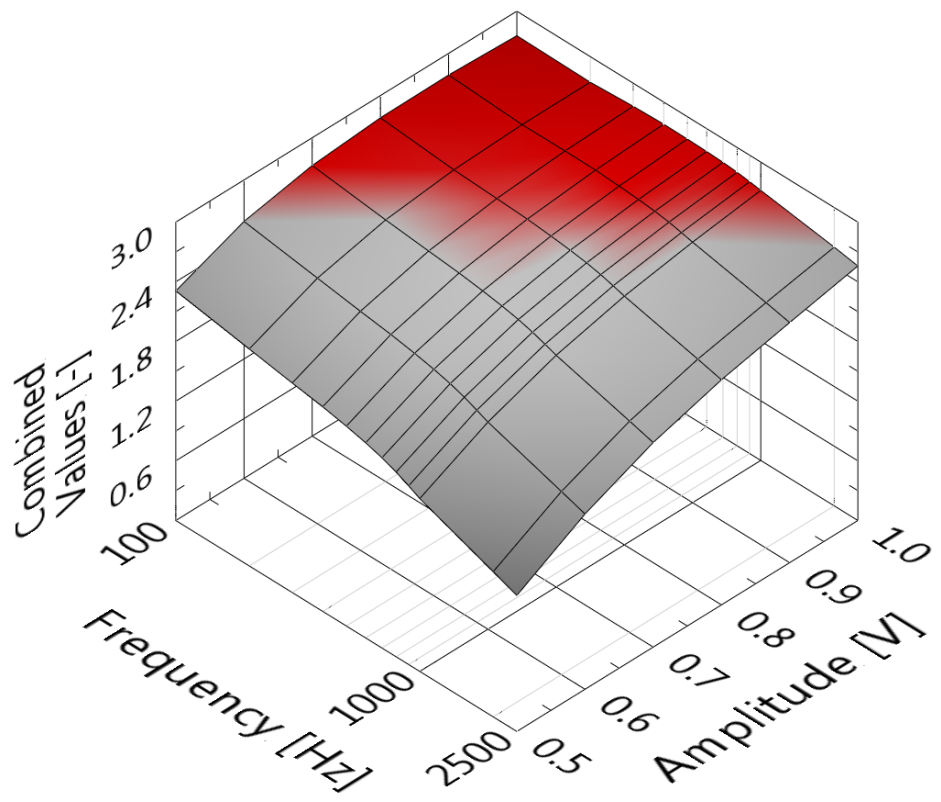

Figure 5.3: Surface indicating the equally-weighted sum of the normalized analysis parameters demonstrating the optimum SI sweep frequency and amplitude of $300 \mathrm{~Hz}$ and $1.0 \mathrm{~V}$. High contour values (highlighted in red) indicate regions of desirable signal attributes. 


\subsubsection{Optimum WMS Laser Driving Signal Frequencies and Amplitudes}

The optimal WMS modulation frequency and amplitude were determined at the center of the $2 v_{3} \mathrm{R}(3)$ methane manifold with no concurrent sweep. An optimal sweep frequency and amplitude were subsequently determined for the chosen modulation parameters. This approach allowed reduction in the size of the required number of parametric measurements and was not expected to strongly affect the outcome, since the absorption and $2 f$ feature maxima were always located and analyzed at the center of the sweep period where changes in SNR, sensitivity, and conformity to theory do not change drastically with sweep signal frequency and amplitude. Furthermore, any influence of residual amplitude modulation (RAM) on the results were found to be inconsequential (Section 5.3.2) when the absorption and $2 f$ values were obtained from the pair-wise average of the leading and lagging features (i.e. the features obtained when the absorption manifold is traversed with an increasing and decreasing wavelength respectively).

Experiments were performed with the optical sensor head coupled to the system with a 1.0 meter optical fiber, with thermal stabilization of the fiber optical components, and using a nitrogen-purged test chamber at ambient atmospheric temperatures and pressures. The methane $2 v_{3} \mathrm{R}(3)$ absorption and second-harmonic signals were calculated using the absorption-free intensity signal estimated from the concurrent intensity correction, and the manifold center was estimated using the reference cell signal maximum.

Figure 5.4 shows sample data (taken at a methane VMR of $10 \mathrm{ppm}_{\mathrm{v}}$ ) illustrating the influence of laser modulation frequency and amplitude on measured $2 f$ value SNR, measurement sensitivity, and correspondence to theory plotted as a percent of their respective maximum values. Results are representative of the trends observed between 2.5 and $50 \mathrm{ppm}_{\mathrm{v}}$. 

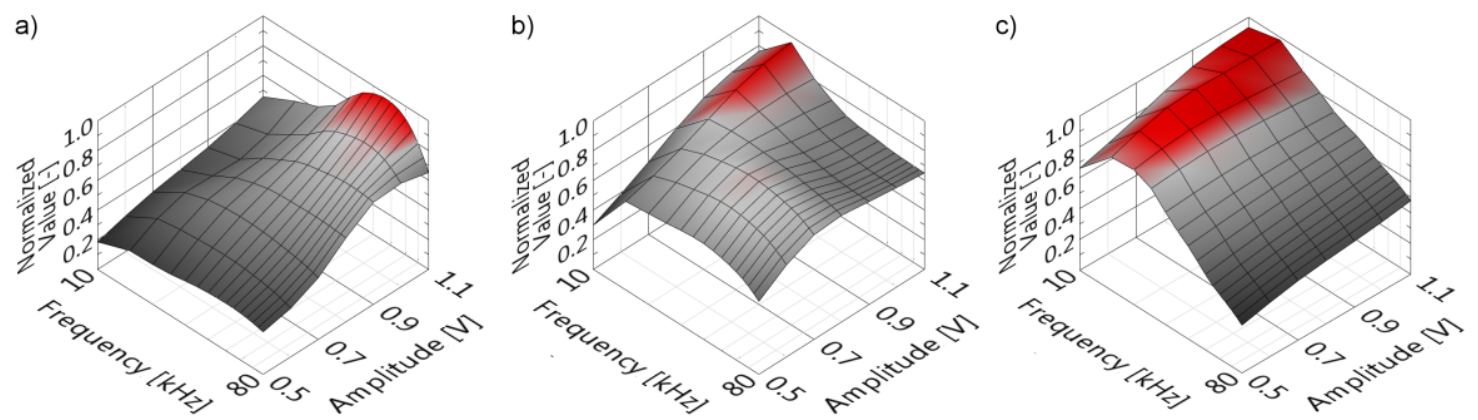

Figure 5.4: Surfaces indicating the normalized values of a) SNR, b) sensitivity, and c) the difference between the theory-based and measured $2 f$ values for the WMS technique (with no wavelength sweep). Data was obtained using the $2 v_{3} \mathrm{R}(3)$ methane manifold for a methane at a volume mixing ratio of 10 ppm $_{v}$ in nitrogen, at standard atmospheric conditions. High contour values (highlighted in red) indicate regions of desirable signal attributes.

The maximum measured $2 f$ SNR occurred at high modulation frequencies and amplitudes, whereas the maximum sensitivity and experimentally measured $2 f$ value matched theory-based estimations most closely at low frequencies and high amplitudes. The equally-weighted sum of the normalized parameters suggests the optimized performance at low modulation frequencies and a high modulation amplitude, as illustrated in Figure 5.5. 


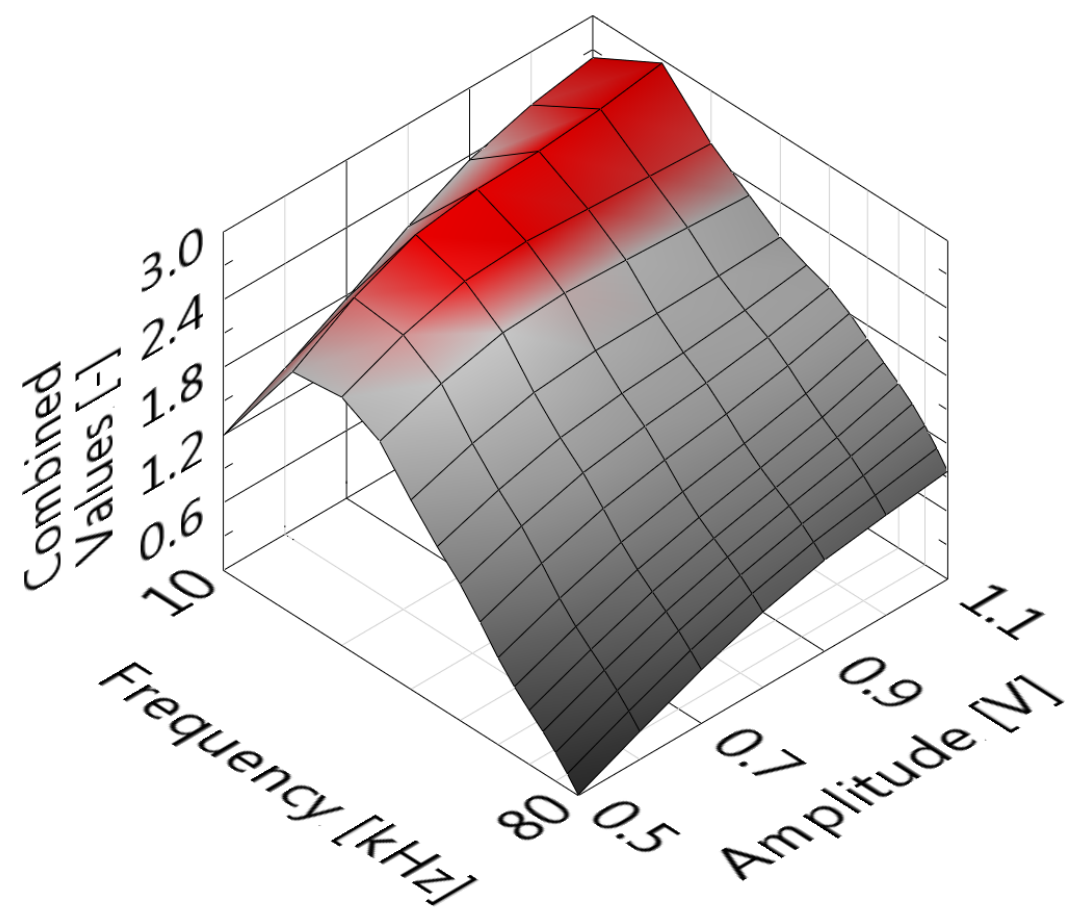

Figure 5.5: Surface indicating the equally-weighted sum of the normalized analysis parameters, demonstrating the optimum WMS signal frequency and amplitude at low frequencies and high amplitudes. High contour values (highlighted in red) indicate regions of desirable signal attributes.

The laser diode limited the maximum permissible modulation amplitude to $1.125 \mathrm{~V}$, though this was only achievable if there was no simultaneous sweep signal. To maximize the resolution of the signal generator, and to permit sufficient sweep signal amplitude, a modulation amplitude of $1.0 \mathrm{~V}$ was selected. A modulation frequency of $20 \mathrm{kHz}$ was selected to ensure the modulation frequency and sample rate $(400 \mathrm{kHz})$ were integer multiples, and to permit a wide array of permissible sweep frequencies which also must be an integer multiple of both of these. The optimum WMS sweep frequency and amplitude were determined at the selected laser driving modulation frequency of $20 \mathrm{kHz}$ and amplitude of $1.0 \mathrm{~V}$. As expected, the optimum WMS sweep frequency and amplitude were only influenced by the measured $2 f$ signal scatter, which in turn appeared to be dominated by the resolution of the $2 f$ feature and the number of points used to calculate the $2 f$ values.

Figure 5.6 illustrates the measured $2 f$ value SNR $(1 \sigma)$ for a 1-s average for a range of sweep amplitudes and frequencies. The peak of the $2 f$ feature was best resolved at low 
WMS sweep frequencies and amplitudes. Based on this result, an optimum sweep frequency of $100 \mathrm{~Hz}$ was selected, a common multiple of the digitizer sample rate $(400 \mathrm{kHz})$ and the modulation frequency $(20 \mathrm{kHz})$. The optimum driving signal amplitude occurred at the minimum possible sinusoidal signal amplitude from the signal generator, $75 \mathrm{mV}$. Table 5.2 lists the SI and WMS laser driving frequencies and amplitudes which yielded the optimum measurement performance based on SNR, sensitivity, and conformity to theory-based estimations.

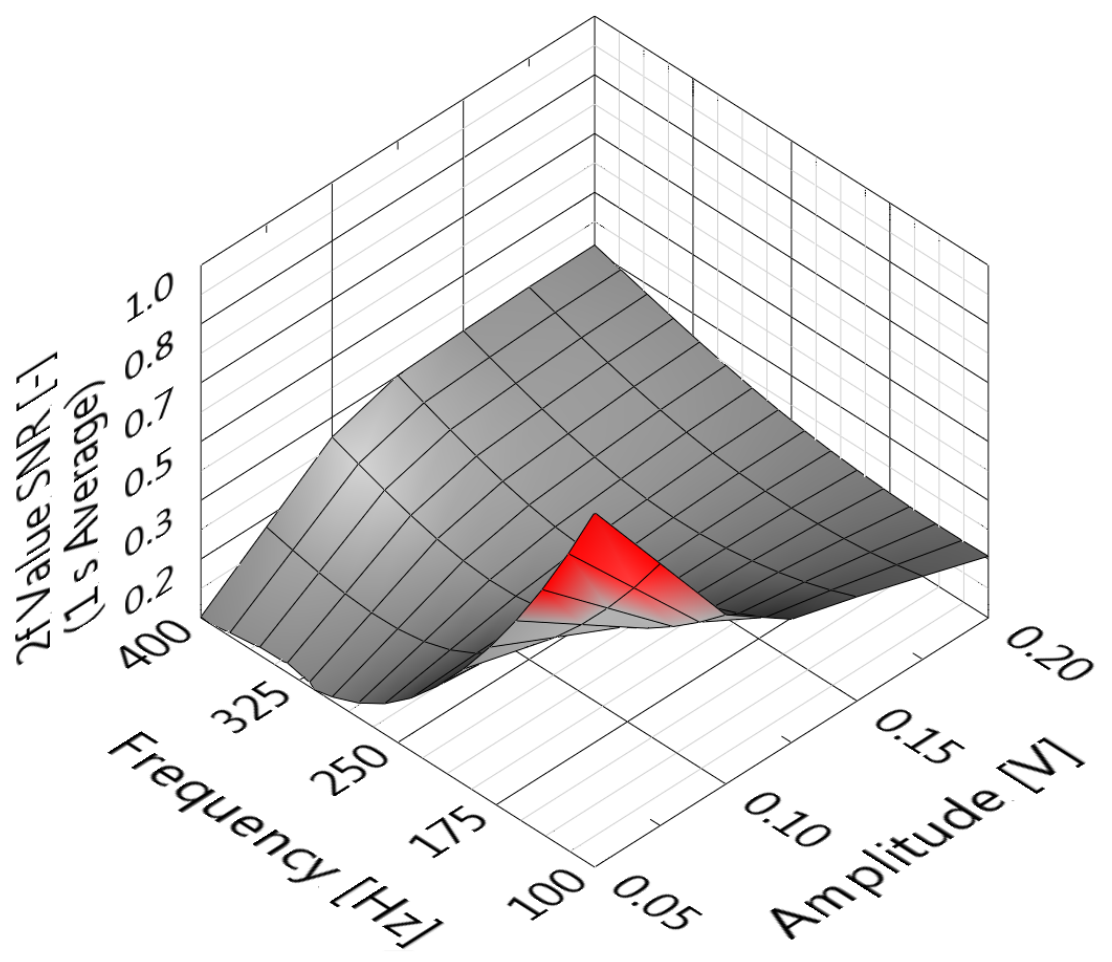

Figure 5.6: Surface indicating $2 f$ value SNR for a one-second average over a range of WMS sweep signal frequencies and amplitudes. Data obtained with the test chamber purged with nitrogen at standard atmospheric conditions. High contour values (highlighted in red) indicate regions of desirable signal attributes. 
Table 5.2: Optimum laser driving signal amplitudes and frequencies for the SI and WMS techniques

\begin{tabular}{c|c|c|c|c}
\hline \multirow{2}{*}{\multicolumn{2}{c|}{ Method }} & \multicolumn{3}{c}{ Optimum Signal Parameter } \\
\cline { 3 - 5 } \multicolumn{2}{c|}{} & \multicolumn{2}{c}{ Amplitude } & Frequency \\
& & {$[\mathrm{V}]$} & {$\left[\mathrm{cm}^{-1}\right]$} & {$[\mathrm{Hz}]$} \\
\hline SI & Sweep & 0.075 & $\sim 0.009$ & 100 \\
\hline \multirow{2}{*}{ WMS } & Sweep & 0.075 & $\sim 0.009$ & 100 \\
& Modulation & 1.0 & $\sim 0.020$ & 20,000 \\
\hline
\end{tabular}

\subsection{Software Analysis Algorithms to Maximize Measurement Precision}

A variety of signal processing methods for minimizing impacts of intensity and wavelength drift, RAM, and optical interference were investigated. As presented in the following sections, the effectiveness of each approach was quantified in terms of its ability to improve methane volume mixing ratio measurement precision and lower detection limit, with calculation details provided in Appendix C.

\subsection{Absorption-Free Intensity Estimate}

An estimate of absorption-free intensity signal is required to generate the SI absorption and WMS $2 f$ signals. In the most basic system configuration, the absorption-free intensity was determined using a pre-run 'calibration', as presented in Section 4.3.1. However, this approach failed to account for subsequent drift in the mean return intensity. This intensity variation is illustrated in Figure 5.7, which plots the percent change in sensor head mean return intensity from the initial pre-run calibration over the course of several hours for four different tests. 


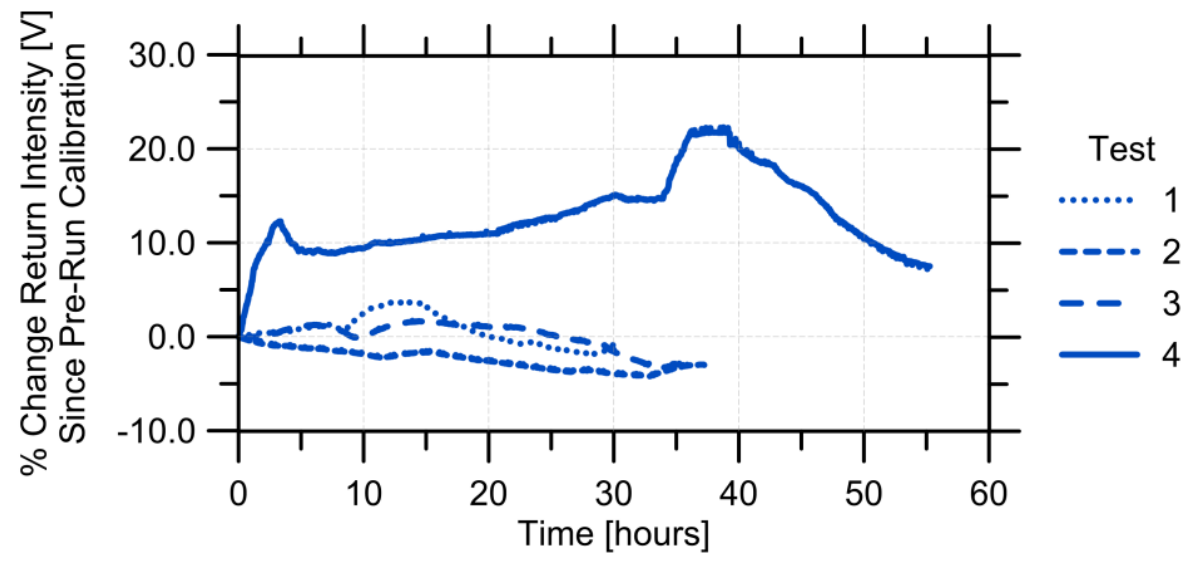

Figure 5.7: Percent change in sensor head return intensity with time, relative to the pre-run calibration intensity. Data obtained with test chamber purged with nitrogen (at near standard ambient pressures and temperatures) while containing the $5.79 \mathrm{~m}$ sensor head, connected to the base station with a 5-m long, 9/125 $\mu \mathrm{m}$ fiber optic cable.

As introduced in Section 4.3.1, both a concurrent intensity correction and a reference measurement using a second (reference) laser were separately implemented in an attempt to mitigate the influence of intensity drift on the estimated absorption-free intensity. Table 5.3 and Table 5.4 list results for the different combinations of absorption-free intensity estimation methods used in conjunction with different calibration methods (Section 4.6 and Appendix B). Details of the computation of $95 \%$ $(2 \sigma)$ precision and LDL are provided in Appendix C. These results were acquired at pressures between 98 and $103 \mathrm{kPa}$, at temperatures between 21 and $27^{\circ} \mathrm{C}$, with the optical sensor head connected to the base TDLAS system via a $6.0 \mathrm{~m}$ SM optical fiber. 
Table 5.3: SI measurement precision at 2 and $50 \mathrm{ppm}_{\mathrm{v}}$ for various calibration and absorption-free intensity estimation methods

\begin{tabular}{|c|c|c|c|c|}
\hline \multirow{2}{*}{$\begin{array}{l}\text { Calibration } \\
\text { Method }\end{array}$} & \multirow{2}{*}{$\begin{array}{l}\text { Absorption-Free } \\
\text { Intensity Estimate }\end{array}$} & \multicolumn{2}{|c|}{ Precision $(2 \sigma)\left[p p m_{v}\right]$} & \multirow{2}{*}{$\begin{array}{c}\text { LDL } \\
{\left[\mathrm{ppm}_{\mathrm{v}}\right]}\end{array}$} \\
\hline & & $\begin{array}{c}\text { At } 2 \\
\text { ppm }_{v}\end{array}$ & $\begin{array}{l}\text { At } 50 \\
\text { ppm }_{v}\end{array}$ & \\
\hline \multirow{3}{*}{$\begin{array}{l}\text { Theory- } \\
\text { based }\end{array}$} & Pre.-Run & 44.9 & 44.7 & 44.3 \\
\hline & Concurrent & 7.5 & 7.8 & 7.8 \\
\hline & Reference Laser & 32.3 & 35.9 & 35.4 \\
\hline \multirow{3}{*}{$\begin{array}{l}\text { Uncorrected } \\
\text { Experimental }\end{array}$} & Pre.-Run & 48.2 & 46.8 & 46.2 \\
\hline & Concurrent & 7.5 & 7.7 & 7.7 \\
\hline & Reference Laser & 37.5 & 37.2 & 36.6 \\
\hline Intensity- & Pre.-Run & 51.2 & 49.2 & 44.1 \\
\hline Corrected & Concurrent & 7.1 & 7.4 & 6.2 \\
\hline Experimental & Reference Laser & 39.2 & 38.7 & 27.8 \\
\hline
\end{tabular}

Table 5.4: WMS measurement precision at 2 and $50 \mathrm{ppm}_{\mathrm{v}}$ for various calibration and absorption-free intensity estimation methods

\begin{tabular}{|c|c|c|c|c|}
\hline \multirow{2}{*}{$\begin{array}{l}\text { Calibration } \\
\text { Method }\end{array}$} & \multirow{2}{*}{$\begin{array}{l}\text { Absorption-Free } \\
\text { Intensity Estimate }\end{array}$} & \multicolumn{2}{|c|}{ Precision $(2 \sigma)\left[p p m_{v}\right]$} & \multirow{2}{*}{$\begin{array}{c}\text { LDL } \\
{\left[p p m_{v}\right]}\end{array}$} \\
\hline & & $\begin{array}{r}\text { At } 2 \\
\text { ppm }_{\mathrm{v}}\end{array}$ & $\begin{array}{l}\text { At } 50 \\
\text { ppm }_{v}\end{array}$ & \\
\hline \multirow{3}{*}{$\begin{array}{l}\text { Theory- } \\
\text { based }\end{array}$} & Pre.-Run & 6.4 & 4.0 & 6.5 \\
\hline & Concurrent & 1.8 & 1.1 & 1.7 \\
\hline & Reference Laser & 2.1 & 1.3 & 2.1 \\
\hline \multirow{3}{*}{$\begin{array}{l}\text { Uncorrected } \\
\text { Experimental }\end{array}$} & Pre.-Run & 10.0 & 8.2 & 10.3 \\
\hline & Concurrent & 9.4 & 7.2 & 9.7 \\
\hline & Reference Laser & 9.4 & 7.1 & 9.7 \\
\hline Intensity- & Pre.-Run & 5.2 & 5.7 & 4.6 \\
\hline Corrected & Concurrent & 2.3 & 1.7 & 2.0 \\
\hline Experimental & Reference Laser & 2.3 & 1.7 & 1.6 \\
\hline
\end{tabular}

The combination of the return-intensity-corrected experimental calibration and concurrent absorption-free intensity estimate resulted in the lowest LDL $\left(6.2 \mathrm{ppm}_{\mathrm{v}}\right)$ and precision value $\left(7.4 \mathrm{ppm}_{\mathrm{V}}\right)$ for the SI method. For the WMS method, the theory-based calibration and concurrent absorption-free intensity estimate resulted in the lowest LDL $\left(1.7 \mathrm{ppm}_{\mathrm{v}}\right)$ and precision value $\left(1.8 \mathrm{ppm}_{\mathrm{v}}\right)$. The most basic signal-processing methods, i.e. the calibration method without return-intensity normalization and the absorption-free intensity estimated using the pre-run calibration, yielded consistently inferior precision and LDL values to the other methods, and were not considered further. 


\subsubsection{Absorption and $2 f$ Feature Center Index}

The SI and WMS methods rely on a stable laser center wavelength $(\bar{v})$ to ensure the manifold absorption peak is captured within the wavelength sweep. Active thermal and current control were used to stabilize the diode as much as possible, however some residual laser diode instability remained, leading to low-frequency noise on the laser output wavelength for the same input voltages. Although some wavelength noise is expected due to electronic noise on the input driving signal, residual noise levels were more likely due to the capability limits of the laser diode thermal control system. The resultant wavelength noise was perceived as a small random low-frequency shift in the location of the absorption or $2 f$ feature maximum from sweep to sweep.

The most basic method of estimating methane volume mixing ratio, the baseline case, extracts absorption or $2 f$ values at a fixed sample index within each sweep period. This method relies heavily on the wavelength stability of the system and can lead to increased precision uncertainties as the peak location of the absorption or $2 f$ feature varies. In an attempt to reduce methane measurement scatter introduced by wavelengthnoise, three post-processing methods were investigated. These methods all use the high SNR reference cell signal and apply various means to determine the index of its feature maximum, which is then used to infer the index of the absorption or $2 f$ feature maximum in the optical sensor head signal (acquired simultaneously, as mentioned previously in Section 3.1.3).

The first method was computationally simple and utilized the index of the maximum reference cell absorption or $2 f$ feature value (ignoring the influence of noise) to infer the index of the absorption or $2 f$ feature maximum in the simultaneously-sampled optical sensor head signal. The second method was identical, except the $2 f$ peak height value in the optical sensor head was calculated as the average of the three values centered about the peak (i.e. average of the $2 f$ peak height at the index location-determined from the reference cell peak height — and the adjacent lower and higher indices). The third method used a least sum squared Gaussian fit of the reference cell absorption or $2 f$ 
feature to precisely locate the index of the peak and then extracted the optical sensor head reading at this same index.

The effectiveness of each technique at reducing the influence of wavelength noise on methane measurement deviation is illustrated in an Allan-Werle diagram in Figure 5.8. The plots show the deviation of the mean measured absorption (Figure 5.8a) and $2 f$ values (Figure 5.8b) for the same dataset as averaging time is increased. The absorption or $2 f$ value standard deviation is plotted for the various methods of estimating the reference cell feature peak position, i.e. inferred at a fixed index of the sweep period (red), at the index corresponding to the reference cell feature maximum (solid green), as a 3-point average of sensor head readings centered about the index of the reference cell feature maximum (green dash), and at the index determined from a Gaussian fit maximum of the reference cell signal (orange). These results were calculated using over 100,000 sweep periods acquired over 12-hours, using the $5.79 \mathrm{~m}$ optical sensor head in the test chamber containing $25 \mathrm{ppm}_{\mathrm{v}}$ methane in air, at ambient temperature and pressure. 
a) SI Method

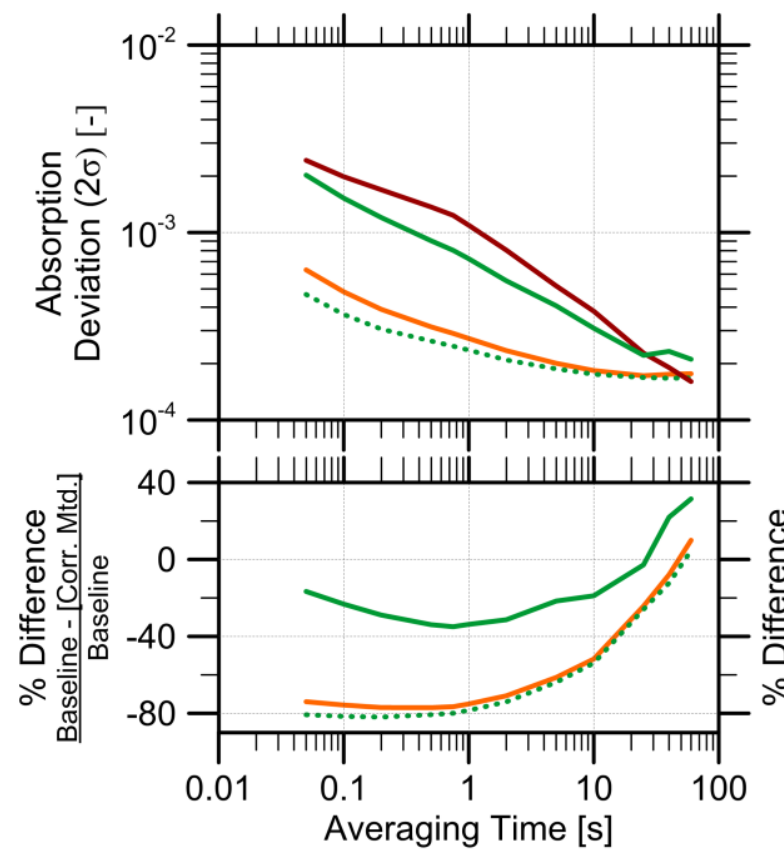

b) WMS Method

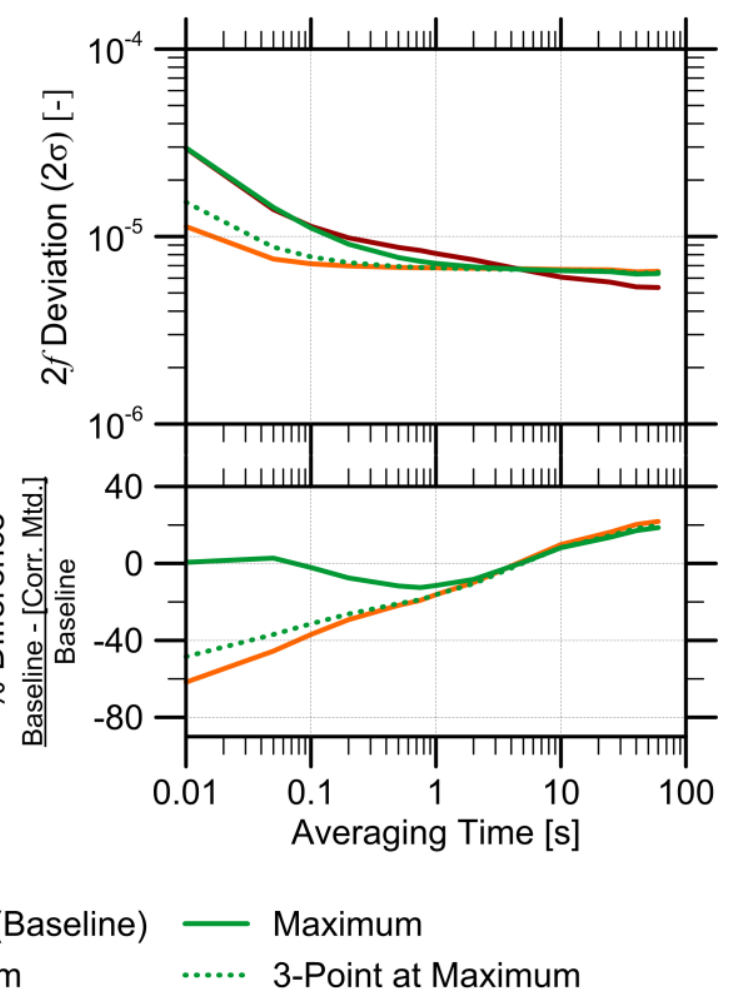

Figure 5.8: SI (a) and WMS (b) optical sensor head measurement deviation versus averaging time for various methods of estimating the index of the reference cell absorption or $2 f$ feature maximum; and their difference relative to using the (baseline) reference cell maximum value. Results calculated using over 100,000 sweep periods acquired over 12hours, with the $5.79 \mathrm{~m}$ optical sensor head in the test chamber containing $25 \mathbf{~ p p m}_{\mathrm{v}}$ methane in air, at standard atmospheric temperature and pressure.

For the SI method the Gaussian fit and 3-point average methods yielded substantially lower deviation than the baseline case of a fixed index value (a reduction of greater than $60 \%$ for a 1 -s averaging time). The WMS results were similar, yielding a reduction of the 1-s averaged $2 f$ value measurement deviation by about $20 \%$ for the 3 point average and Gaussian fit methods. Overall, the 3-point average and Gaussian fit yielded the lowest deviation of the mean measured absorption and $2 f$ values for averaging times of $1 \mathrm{~s}$, with consistent results for tested methane concentrations between 2 and $50 \mathrm{ppm}_{\mathrm{v}}$ in air, near STP. For longer averaging times, these methods of determining the index of feature maxima were no longer advantageous, and use of a constant index yielded the lowest absorption and $2 f$ value deviation. 
The influence of these techniques on system measurement performance is illustrated in Figure 5.9, which plots the maximum measurement precision value between 2.0 and $50 \mathrm{ppm}_{\mathrm{v}}$ for the theory-based and experimental calibration methods, and the concurrent correction or reference laser absorption-free intensity estimation methods. These results were calculated using the same dataset (acquired over the course of 8 hours and includes over 600 one-second averages) with test chamber methane-in-air concentrations between 2.0 and $50.0 \mathrm{ppm}_{\mathrm{v}}$, at pressures between 98 and $103 \mathrm{kPa}$, at temperatures between 21 and $27^{\circ} \mathrm{C}$, and with the optical sensor head connected to the base TDLAS system via a $6.0 \mathrm{~m} \mathrm{SM}$ optical fiber.

a) SI Precision $(2 \sigma)$
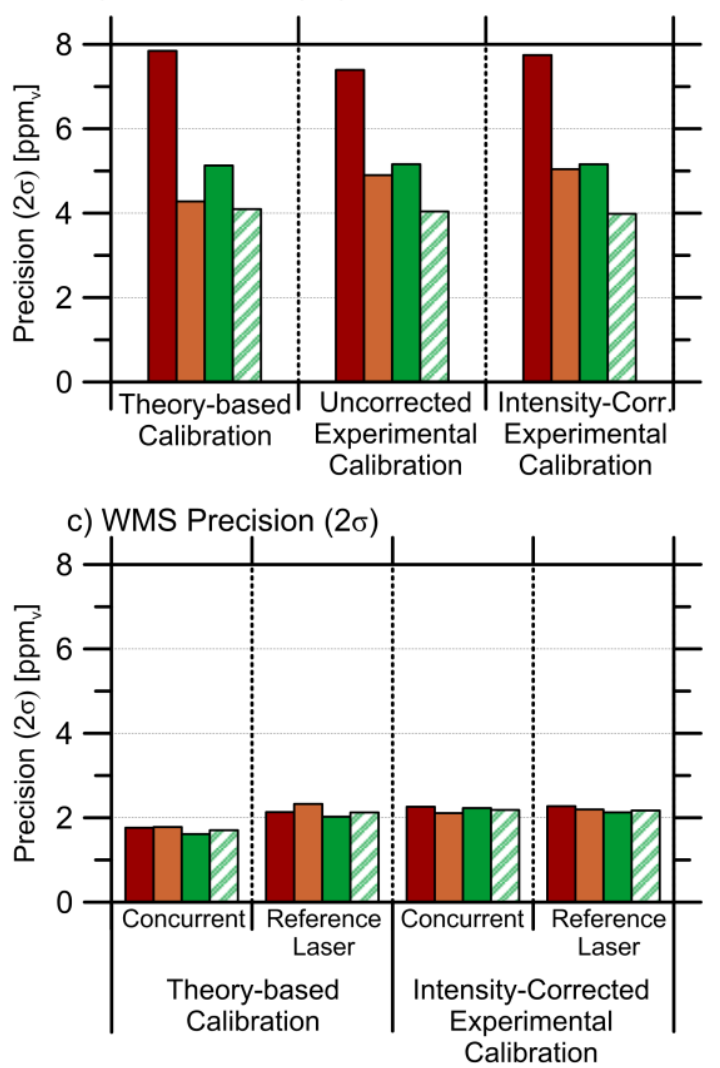

b) SI LDL $(2 \sigma)$

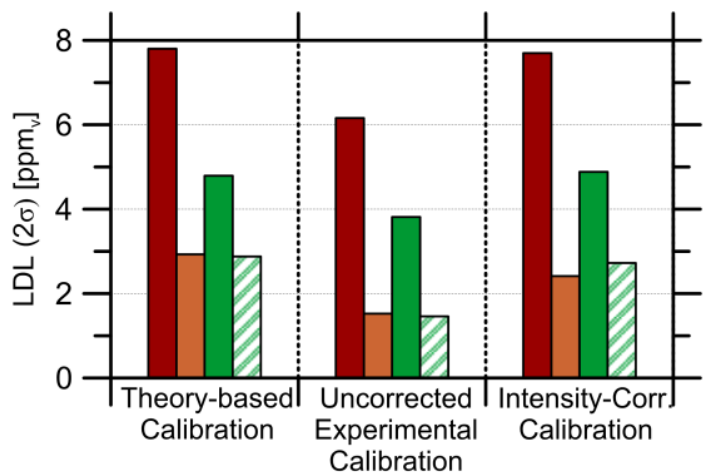

d) WMS LDL $(2 \sigma)$

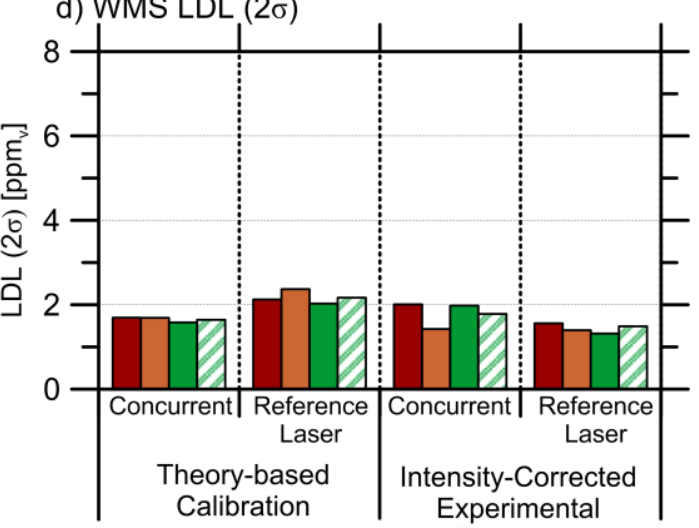

Calibration

Sweep Center Index Gaussian Fit Maximum

Maximum

3-Point Average at Maximum

Figure 5.9: SI (top) and WMS (bottom) 1-second averaged optical sensor head measurement precision $(2 \sigma)$ and LDL for various combined methods of calibration and estimating the index of the reference cell $2 f$ or absorption feature maximum. Parameters calculated using over 600 measurements obtained at test chamber methane-in-air concentrations between 2.0 and $\mathbf{5 0 . 0}$ ppm $_{v}$, acquired over a time-period spanning 8 hours. 
The 3-point average and Gaussian fit methods of estimating the absorption feature center for the theory-based calibration methods returned the lowest $95 \%$ precision values for the SI technique, at 4.1 and $4.3 \mathrm{ppm}_{\mathrm{v}}$ respectively. The 3-point average and Gaussian fit methods combined with the intensity-corrected experimental calibration method produced the lowest overall LDL of $1.5 \mathrm{ppm}_{\mathrm{v}}$ for both. For the WMS method, the concurrent absorption-free intensity estimate combined with the theory-based calibration and the reference cell maximum method yielded the lowest values of precision $\left(1.6 \mathrm{ppm}_{\mathrm{v}}\right)$, and LDL of $\left(1.6 \mathrm{ppm}_{\mathrm{v}}\right)$, but was closely followed by the 3-point average method which yielded a precision of $1.7 \mathrm{ppm}_{\mathrm{v}}$ and LDL of $1.6 \mathrm{ppm}_{\mathrm{v}}$.

Generally, at the desired 1-s averaging time for the target fugitive emissions measurement application, the measurement results using the 3-point average method of estimating the index of the reference cell maximum returned the most desirable LDL and prediction intervals. Similar performance was achieved using the Gaussian fit method, but this required significantly longer computation times. For this reason the 3-point average method of determining the index of the reference cell absorption and $2 f$ feature maximum was implemented in subsequent tests.

\subsubsection{Digital RAM Subtraction}

The influence of software removal of RAM on methane measurement precision using the SI and WMS methods was investigated. As discussed in Section 2.2.2, RAM is the inherent variation in laser intensity with wavelength, such that as the wavelength is swept over an absorption feature of interest there is an associated intensity variation, $I_{0_{s}}(\lambda)$. This complicates the estimation of the absorption-free intensity $I_{0_{S}}{ }_{s}$, which cannot be directly measured because of absorption by the target species of interest, and must instead be approximated as discussed in Section 4.3.1. Section 2.2.2 suggested measurement error due to RAM increases as methane volume mixing ratio decreases. Fortunately, the estimate of $I_{0_{S}}{ }^{*}$ improves as absorption decreases, as discussed in Section 4.3.1.2.

Figure 5.10 demonstrates the influence of averaging time on measurement standard deviation $(2 \sigma)$ of the leading absorption and $2 f$ values (inferred from the features 
generated while injection current and wavelength are increasing in each sweep period, Section 4.5), with and without digital RAM subtraction. The plotted results used the 3point-average method for determining the absorption or $2 f$ feature maximum, and were observed to be similar to the results obtained using the lagging feature. These results were obtained using the optimum laser driving signals and the $5.79 \mathrm{~m}$ optical sensor from the test chamber containing $2,5,10,25$, and $50 \mathrm{ppm}_{\mathrm{v}}$ methane in air at atmospheric pressure and temperature. The remote sensor head was coupled to the base system with a $6.0 \mathrm{~m} \mathrm{SM}$ optical fiber. This dataset is comprised of at least 800 seconds of absorption and $2 f$ data at each methane VMR, acquired over a 12-hour period.
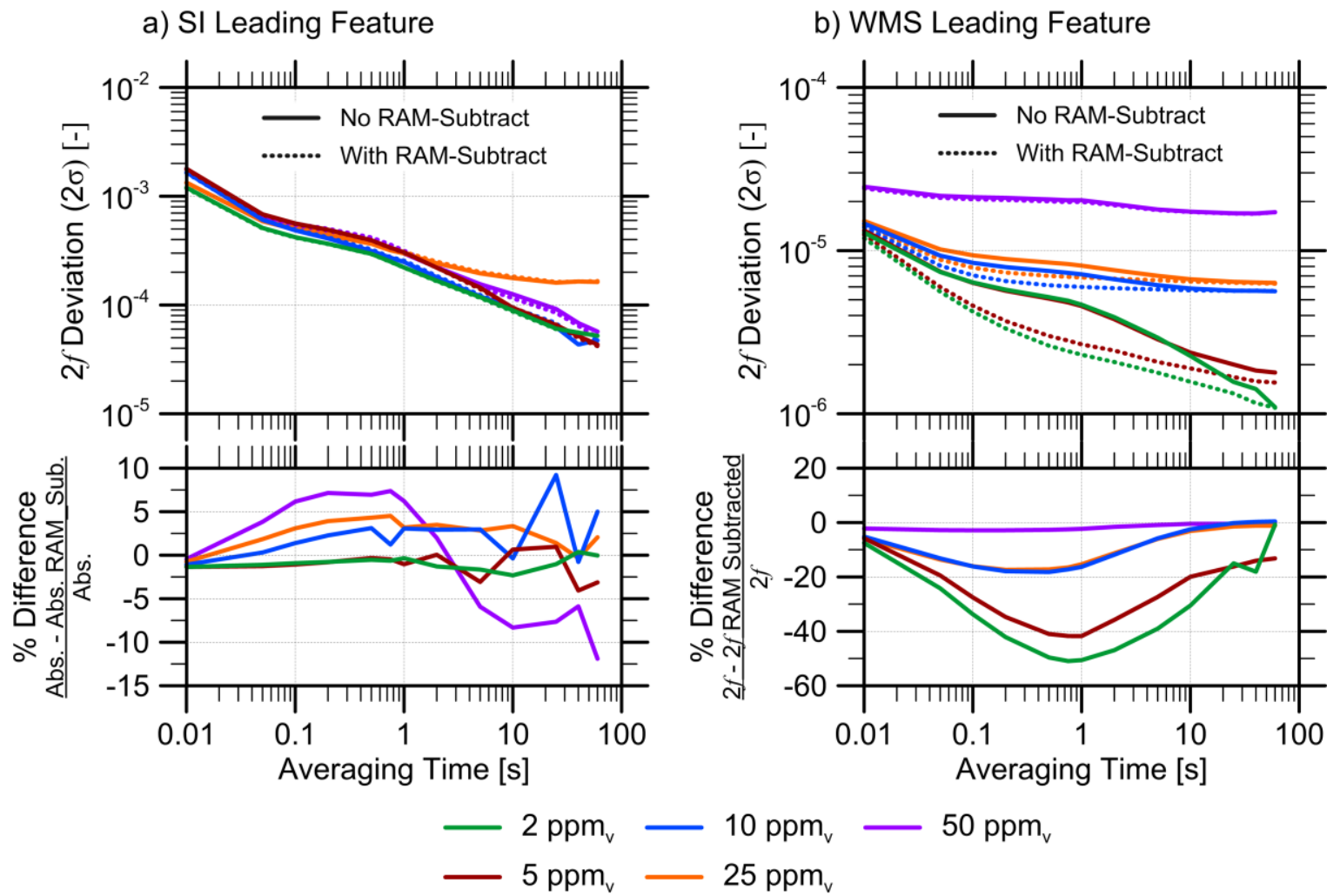

Figure 5.10: The difference in optical sensor head measurement deviation (2 $\sigma)$ for the leading $2 f$ and absorption features with and without digital RAM subtraction for the WMS (a) and SI (b) measurement techniques. This dataset is comprised of at least 800 seconds of absorption and $2 f$ data at each methane volume mixing ratio, each acquired over a 12-hour period.

Digital RAM subtraction was not beneficial at reducing measurement scatter for the SI method, but rather increased measurement deviation by up to $10.0 \%$ for all 
averaging times. However, digital RAM subtraction reduced $2 f$ value deviation for all tested concentrations, yielding the most significant reduction in measurement deviation of $50 \%$ at $2 \mathrm{ppm}_{\mathrm{v}}$ for an averaging time of $1 \mathrm{~s}$.

Driving the laser injection current with a sinusoidal signal rather than a more common ramp function (Werle et al., 1994; Werle et al., 1993; Rieker et al., 2009), permits an alternative means of RAM suppression. Once stabilized, the laser output wavelength can be approximated by a sinusoid (Equation 2.16), with a mean equal to the wavelength of the $2 v_{3} \mathrm{R}(3)$ methane absorption feature. Sinusoidal modulation yields two absorption or $2 f$ features for every sweep period, once with an increasing wavelength (leading) and once with a decreasing wavelength (lagging). The feature maxima occur at integer multiples $(\ell \in \mathbb{N})$ of $\pi f_{s} t$, with the leading and lagging signals at $(2 \ell+1) \pi f_{s} t$ and $(2 \ell) \pi f_{s} t$ respectively, as illustrated in Figure 5.11. In addition to the sinusoidal wavelength variation, modulation of the laser driving current imparts a phase shifted (dominant first-order: $\psi_{0_{s}}$ ) intensity variation at the same frequency. The phase shift between laser output intensity and wavelength increases and decreases the intensity at the leading and lagging absorption and $2 f$ feature peaks respectively. By pair-wise averaging the absorption or $2 f$ feature leading and lagging maximum values in each sweep period, the influence of RAM is cancelled out.

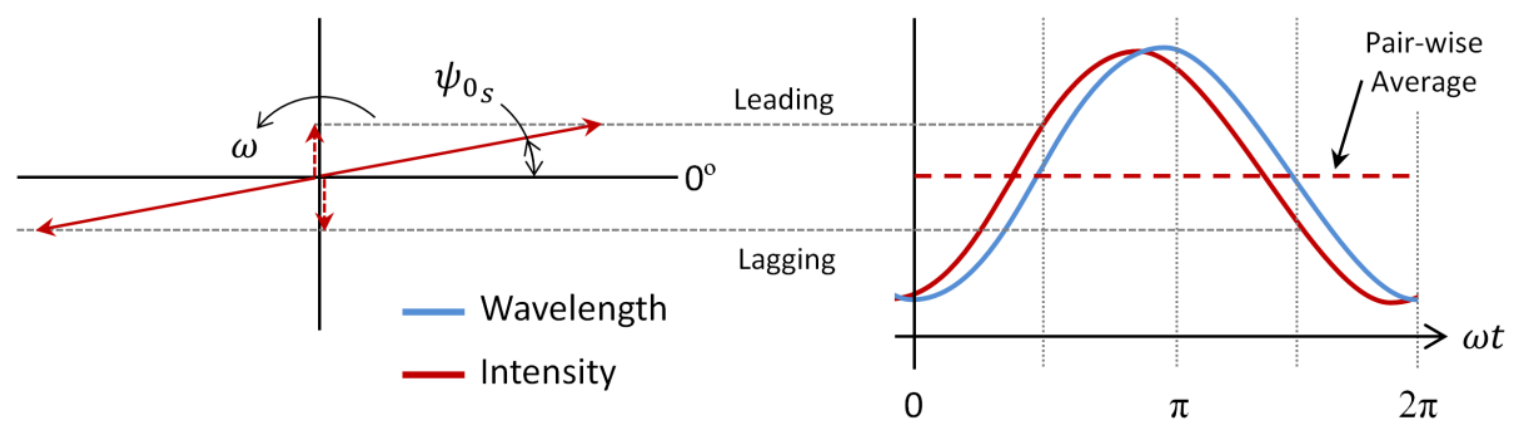

Figure 5.11: Inherent RAM suppression when using the leading and lagging portions of the returning intensity signal

Figure 5.12 compares the measurement precision and LDL without any RAM correction, with digital RAM subtraction, and with RAM suppression using the leading and lagging feature pair-wise averaging method. This study uses a single dataset, 
comprised of over 700 SI and WMS measurements averaged for $1 \mathrm{~s}$, acquired with the $5.79 \mathrm{~m}$ sensor head in the test chamber containing methane concentrations between 2 and $50 \mathrm{ppm}_{\mathrm{v}}$ at near standard atmospheric temperature and pressure.
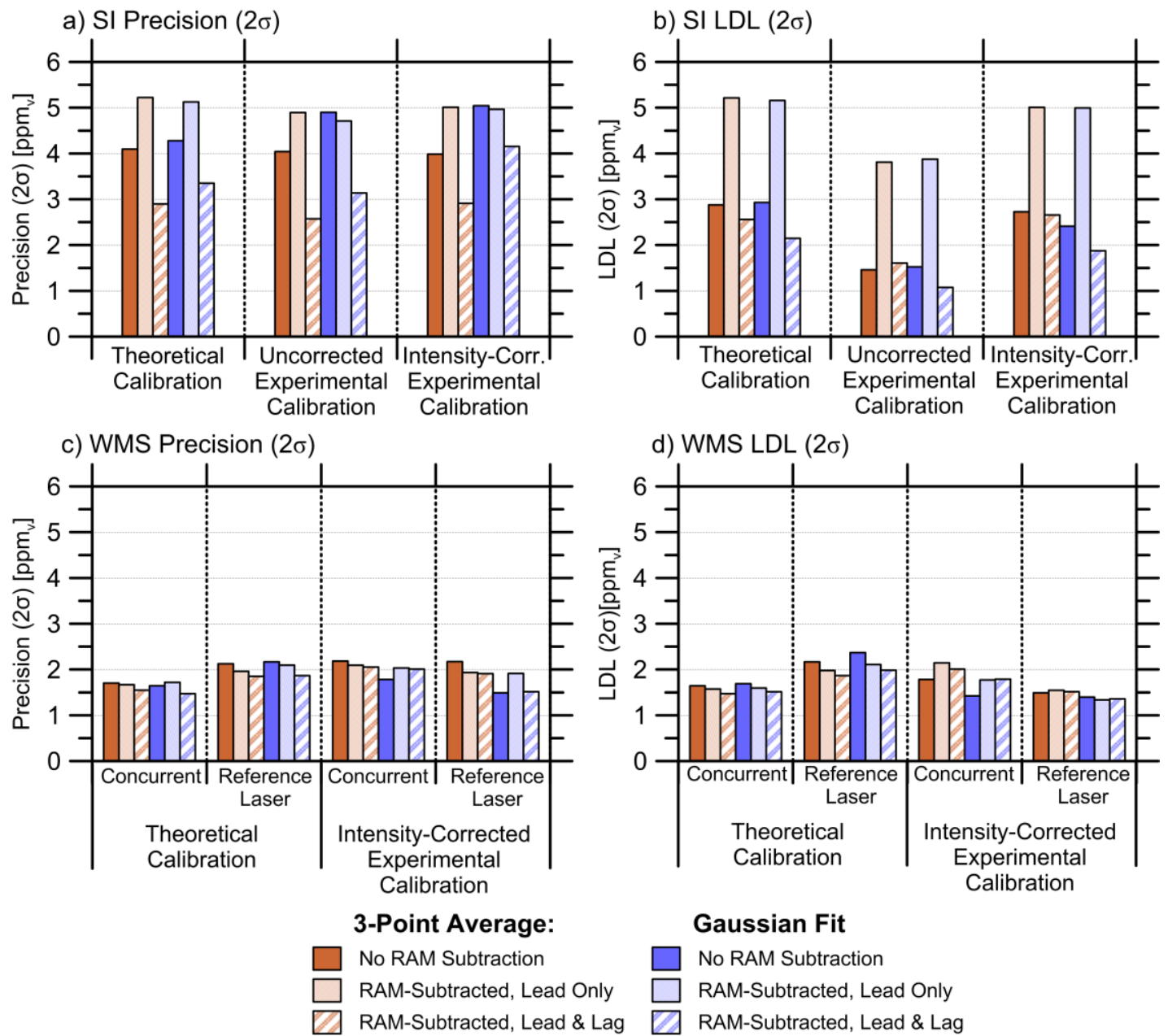

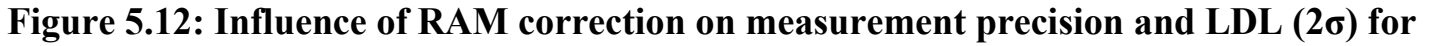
various calibration and absorption-free intensity estimation methods. Data acquired with the $5.79 \mathrm{~m}$ sensor head and test chamber methane concentrations between 2 and $100 \mathbf{~ p p m}_{\mathrm{v}}$ at ambient atmospheric temperature and pressure.

Generally, pair-wise averaging of the leading and lagging absorption or $2 f$ values is superior at reducing measurement precision and LDL values than digital RAM subtraction when only the leading feature is used. For this reason the pair-wise averaging method of RAM suppression was implemented in subsequent tests when noted. 


\subsection{Hardware Configurations to Maximize Measurement Precision}

\subsubsection{Mitigation of Polarization-Dependant Intensity Drift}

Variations in component temperature or fiber strain leads to fluctuations in the observed return intensity, which can be partially attributed to polarization-dependant loss and dispersion (Hecht, 2002; Collett, 2003). This is further complicated if the temperature of the optical components are fluctuating with respect to each other, creating unrepeatable system intensity losses which are not readily quantified. In an effort to reduce the influence of polarization and temperature variation on SI and WMS methane volume mixing ratio measurements, a thermal enclosure was added to the base system and the use of a polarization scrambler was investigated.

Optical components most susceptible to temperature fluctuations were housed within a temperature-regulated enclosure. The temperature within the enclosure was controlled with a thermoelectric heating and cooling system (Section 3.1.3). Polarization scramblers are commonly used in high-speed optical communication systems to reduce polarization-dependant effects by introducing a random polarization within the fiber network (Collett, 2003). The result is an increased perceived intensity variation, which reduced to a common perceived mean intensity when time-averaged (as the degree of polarization of the resultant signal approaches zero).

The effects of polarization scrambling and thermal stabilization on measured absorption and $2 f$ value deviation using the aforementioned optimum laser driving signals, are illustrated in the Allan-Werle deviation plot in Figure 5.13 and Figure 5.14. These plots demonstrate measured optical sensor head absorption and $2 f$ value deviation $(2 \sigma)$ obtained using the optimum laser driving signals and the $5.79 \mathrm{~m}$ optical sensor within a nitrogen-purged test chamber at ambient pressures and temperatures, coupled to the base system with a $6.0 \mathrm{~m} \mathrm{SM}$ optical fiber. The thermal enclosure is maintained at a constant $21.10^{\circ} \mathrm{C}$ when activated, maximizing the $2 f$ signal sensitivity to changes in methane volume mixing ratio within the fiber-coupled optical sensor, and ensuring efficiency and stability of the thermal control system. 


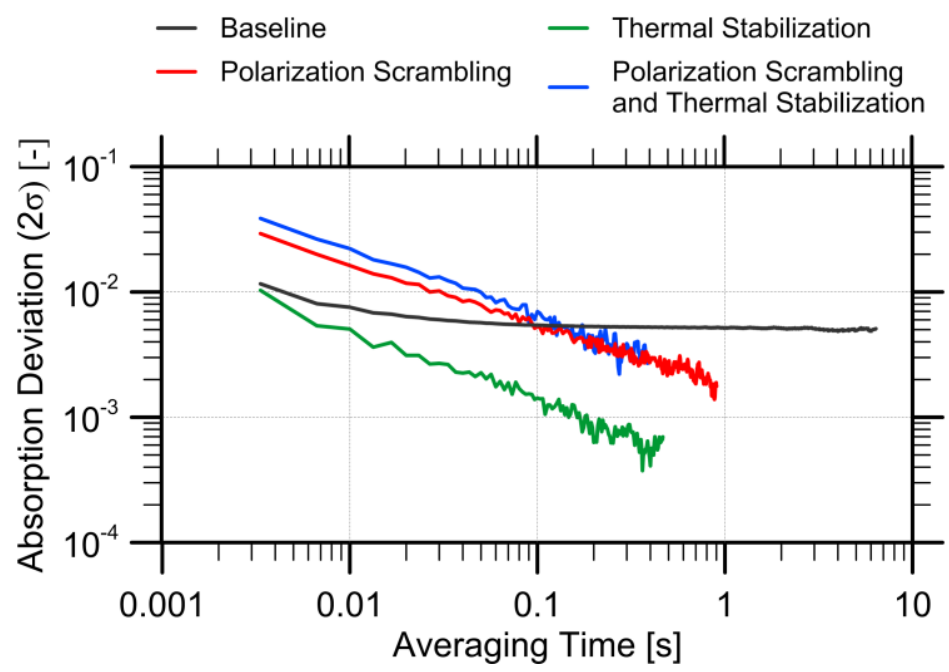

Figure 5.13: Measured absorption value deviation with and without intensity stabilization

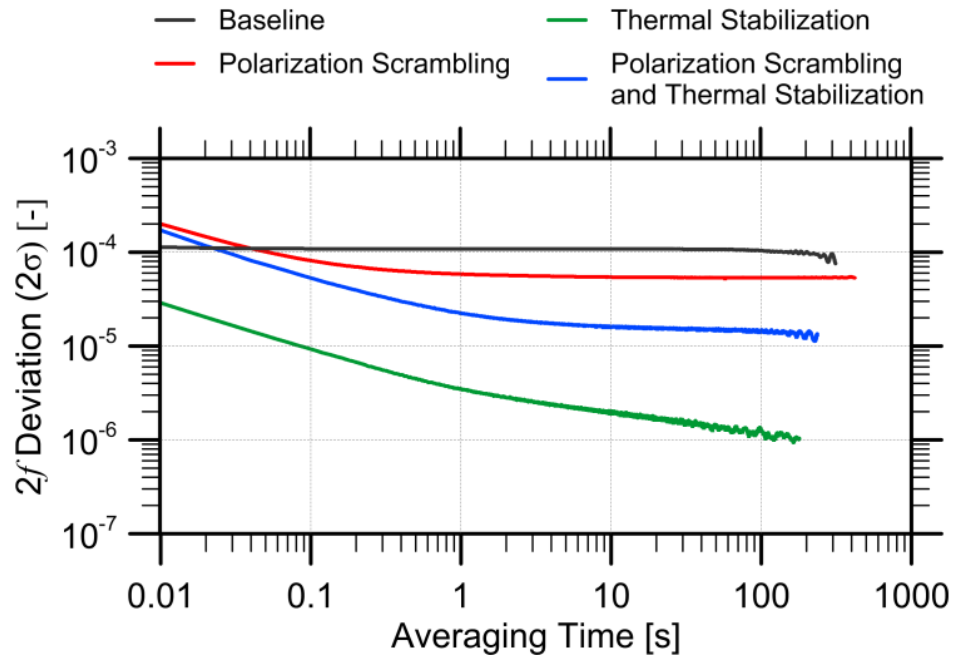

Figure 5.14: Measured $2 f$ value deviation with and without intensity stabilization

When compared to the baseline case, the addition of the polarization scrambler, as expected, increased the instantaneously measured absorption and $2 f$ value deviations, but reduced the measurement deviations with averaging times greater than 0.2 and $0.05 \mathrm{~s}$ respectively. Thermal stabilization with no polarization scrambling yielded the lowest measurement standard deviations, which were consistently lower than those achieved with the polarization scrambler. Interestingly, the positive effects of thermal stabilization on the measured absorption and $2 f$ value deviations were reduced when combined with polarization scrambling. This may be attributable to the piezoelectric transducers within 
the polarization scrambler that impart polarization and amplitude modulation at four fixed frequencies (each less than $700 \mathrm{kHz}$ ). This in-turn would influence the measured signal deviation at the sweep and modulation frequencies of $100 \mathrm{~Hz}$ and $20 \mathrm{kHz}$, which may not be fully suppressed with signal averaging.

Even though long averaging times yielded the lowest signal deviation, these times are not useful for the intended application of observing transient ambient methane plumes, or in a case where sensors are multiplexed and must be sequentially interrogated. Averaging optical measurements for $1 \mathrm{~s}$ balances the trade-offs between minimizing measurement duration and increasing measurement precision by reducing random scatter.

To illustrate typical measurement deviation and scatter, Figure 5.15 plots normalized remote sensor head absorption and $2 f$ values for system configurations with and without thermal stabilization and polarization scrambling. The displayed 1-s averaged results were acquired at requested test chamber methane volume mixing ratios between $2.0 \mathrm{ppm}_{\mathrm{v}}$ and $25.0 \mathrm{ppm}_{\mathrm{v}}$, at pressures between $98 \mathrm{kPa}$ and $103 \mathrm{kPa}$, and at temperatures between $21^{\circ} \mathrm{C}$ and $27^{\circ} \mathrm{C}$. The tests were conducted with the optical sensor head connected to the base TDLAS system via a $6.0 \mathrm{~m} \mathrm{SM}$ optical fiber. 
a) SI Absorption

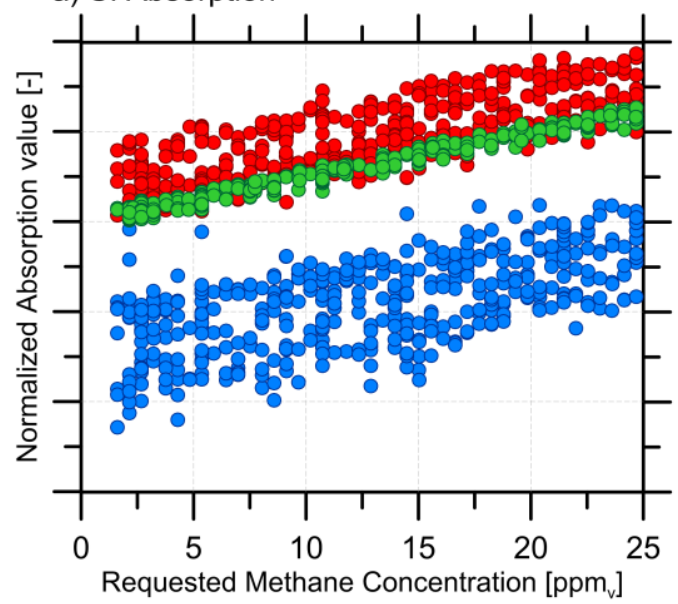

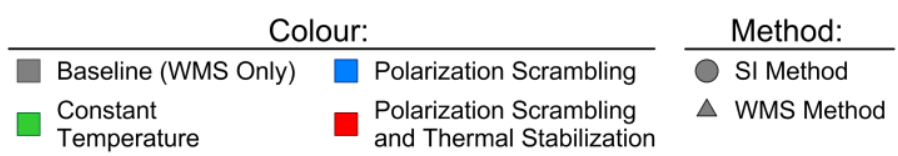

b) WMS $2 f$

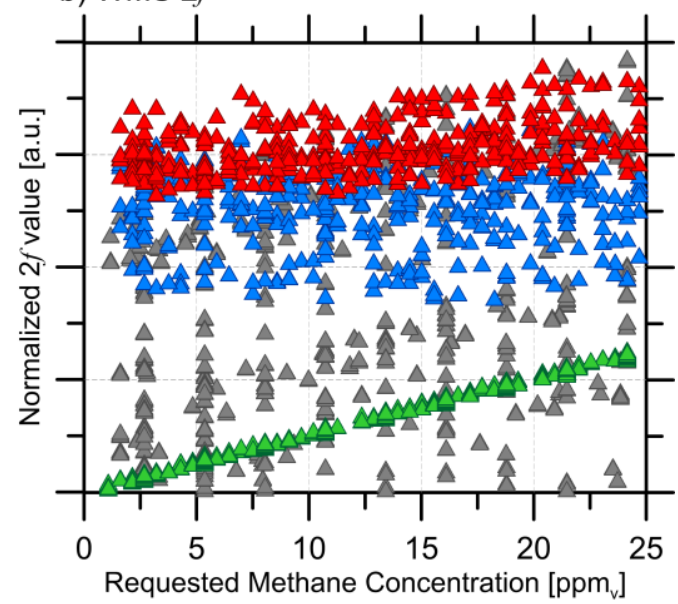

Figure 5.15: Plot of normalized a) SI absorption and b) WMS $2 f$ values with methods of mitigating polarization-dependant intensity fluctuations. Data obtained with test chamber methane concentrations between 2 and $25 \mathbf{p p m}_{\mathrm{v}}$ (at near standard ambient pressures and temperatures) while containing the $5.79 \mathrm{~m}$ sensor head, connected to the base station with a 5 m 9/125 $\mu \mathrm{m}$ fiber optic cable.

A meaningful baseline measurement of absorption could not be acquired for the SI measurement due to extreme variability in the results. However, implementation of the polarization scrambler reduced the standard deviation of the measured WMS- $2 f$ methane VMR values by $43 \%$ (at a 1-s averaging time) compared to the baseline case, but was not as effective as thermal stabilization alone which reduced the standard deviation by $93 \%$. For this reason, thermal stabilization of the fiber-based optical components (without polarization scrambling) was selected to mitigate thermal- and polarization-dependant intensity variation in this system.

\subsubsection{Effects of Fiber-Length on Measurement Accuracy}

When deployed around an industrial facility, the optical sensor heads could require placement up to $1 \mathrm{~km}$ away from the base TDLAS system. To determine the effect of optical fiber length on measured $2 f$ values, laboratory tests were conducted with $5 \mathrm{~m}$,

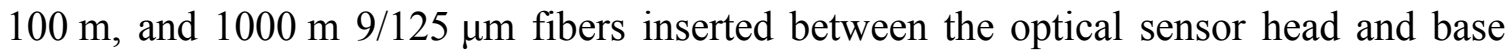
TDLAS system. The addition of the fibers was the only difference in the otherwise 
identical hardware, operating, and ambient conditions. Figure 5.16 and Figure 5.17 illustrate the measured precision and drift of the mean methane in air volume mixing ratio at $2.0 \mathrm{ppm}_{\mathrm{v}}$ and $50.0 \mathrm{ppm}_{\mathrm{v}}$, for the $2 v_{3} \mathrm{R}(3)$ methane manifold at ambient room temperature and pressure.

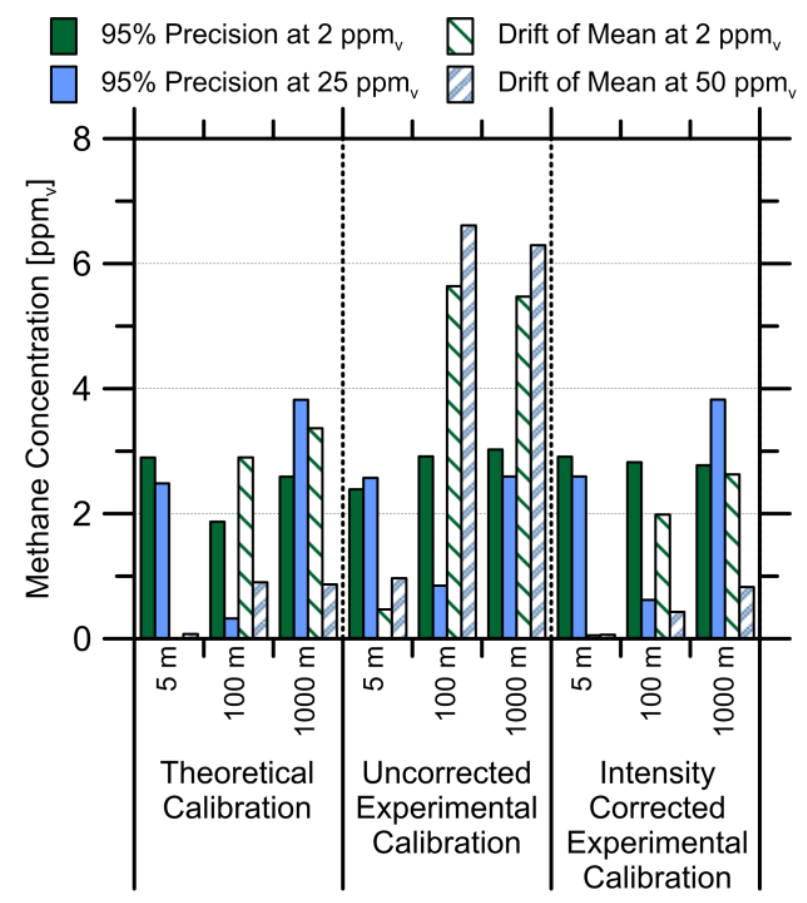

Figure 5.16: Influence of 9/125 $\mu \mathrm{m}$ fiber-length on measured methane volume mixing ratio precision and mean drift for the SI method, using the concurrent method for estimating absorption-free intensity, at the $2 v_{3} \mathrm{R}(3)$ methane manifold at atmospheric temperature and pressure. 


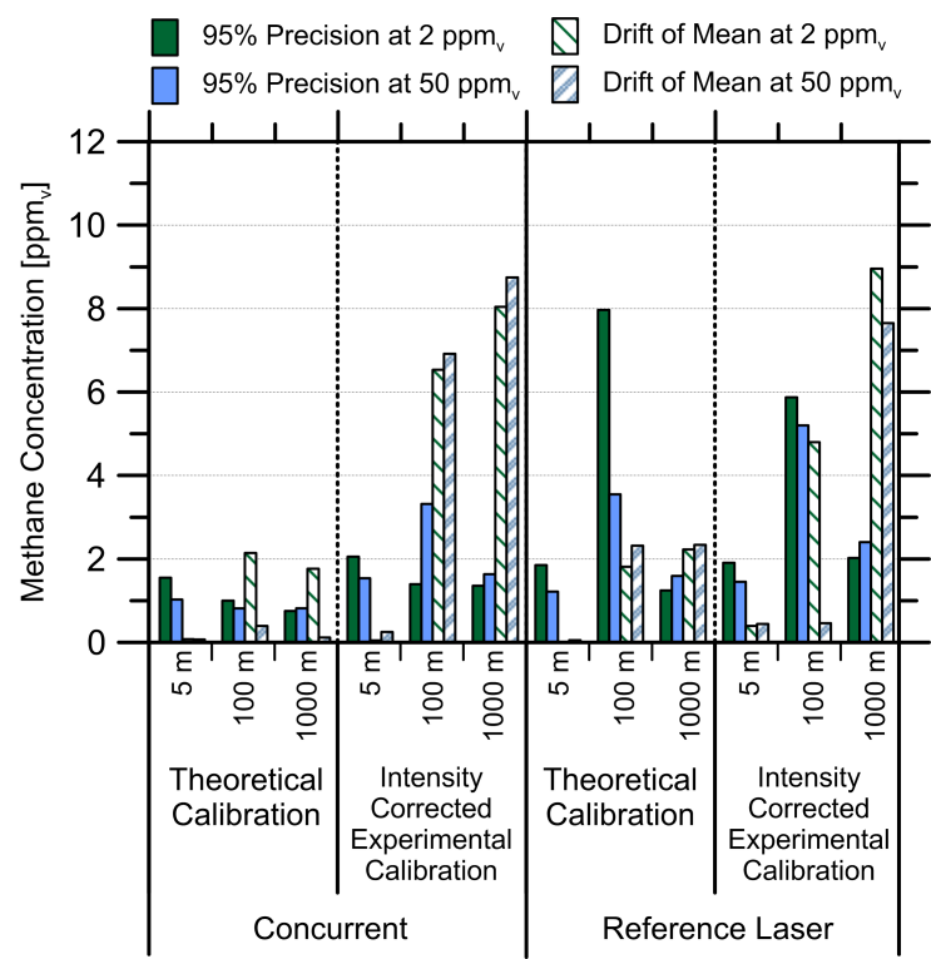

Figure 5.17: Influence of 9/125 $\mu \mathrm{m}$ fiber-length on measured methane volume mixing ratio precision and mean drift for the WMS method, using the concurrent and reference laser methods for estimating absorption-free intensity, at the $2 v_{3} \mathbf{R}(3)$ methane manifold at atmospheric temperature and pressure

The methane measurement precision at $2 \mathrm{ppm}_{\mathrm{v}}$ (solid bars) was relatively consistent for the SI method among the tested fiber lengths and calibration methods, varying by only slightly more than the methane volume mixing ratio uncertainty from the gas mixing system. By contrast, for the WMS modulation scheme, the methane measurement precision varied greatly among the different signal processing and fiber lengths tested. The drift of the mean measurement (hatched bars) increased with increasing fiber length for both the SI and WMS technique, suggesting that fiber length would need to remain fixed after system commissioning. However, so long as the initial calibration was performed using lengths specific to the installation (or using sufficiently long fibers to accommodate varying optical network configurations), the bias could be eliminated. 


\subsection{Achieved Performance}

\subsubsection{Quantified Precision and Lower Detection Limits}

The most basic system configurations (implementing the optimum laser driving signals, thermal stabilization of the fiber optic components within the base-station, and mechanical jitter of the optical sensor head) used a calibration method without returnintensity normalization, possessed no RAM suppression, estimated the absorption-free intensity using the pre-run calibration, and did not account for wavelength drift using the reference cell absorption or $2 f$ feature. Table 5.5 compares the performance of the most basic SI and WMS system configurations (baseline) to the performance required (introduced in Section 2.1.3) to quantify ambient methane.

Table 5.5: Methane measurement performance of the baseline SI and WMS system configurations compared to the required performance

\begin{tabular}{l|r|c|r|c}
\hline & \multicolumn{2}{|c|}{ Precision } & \multicolumn{2}{c}{ LDL } \\
& {$\left[\mathrm{ppm}_{\mathrm{v}}\right]$} & {$\left[\mathrm{cm}^{-1}\right]$} & {$\left[\mathrm{ppm}_{\mathrm{v}}\right]$} & {$\left[\mathrm{cm}^{-1}\right]$} \\
\hline Required & 2.0 & $8.10 \times 10^{-7}$ & 1.75 & $7.09 \times 10^{-7}$ \\
Baseline SI & 47.8 & $1.94 \times 10^{-5}$ & 39.3 & $1.59 \times 10^{-5}$ \\
Baseline WMS & 10.9 & $4.41 \times 10^{-6}$ & 10.8 & $4.36 \times 10^{-6}$ \\
\hline
\end{tabular}

In an effort to reach the required measurement performance, hardware and software based system improvements were implemented. Figure 5.18 summarizes the incremental improvements in measurement performance for the SI method with each additional system enhancement. 


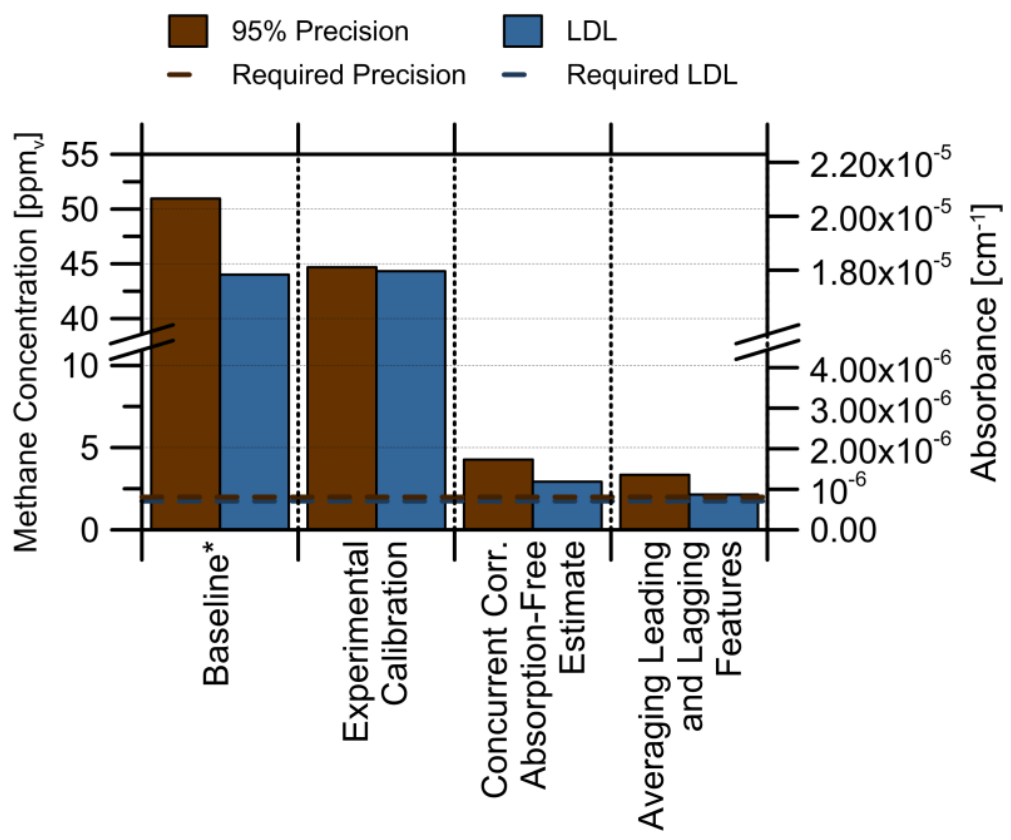

Figure 5.18: Measurement performance improvements for a SI-based system for various hardware and software system enhancements, for the $2 v_{3} R(3)$ methane manifold at ambient temperature and pressure.

Use of an intensity-corrected experimental calibration resulted in marginal improvements in the measurement precision and LDL for the SI method. However, with the addition of the concurrent intensity correction method of estimating the absorptionfree intensity, measurement precision and LDL improved the precision by $89 \%$ and LDL by $96 \%$. Extracting absorption signals from the pair-wise average of the leading and lagging absorption features to reduce the effects of RAM, improved measurement precision and the LDL by $36 \%$ and $29 \%$ respectively; resulting in the final best-case precision and LDL of $3.14 \mathrm{ppm}_{\mathrm{v}}$ and $1.1 \mathrm{ppm}_{\mathrm{v}}\left(1.27 \times 10^{-6} \mathrm{~cm}^{-1}\right.$ and $\left.4.35 \times 10^{-7} \mathrm{~cm}^{-1}\right)$ for the SI method. Unfortunately, even with the system-enhancements presented above, the SI method was not able to achieve the required measurement precision with a 1-s averaging time. Longer averaging times may improve these results further at the expense of slower spatial multiplexing loop-rates.

Figure 5.19 summarizes the incremental improvements in measurement performance for the WMS-based system with each additional system enhancement. The theory-based calibration (which takes in to account ambient temperature and pressure, 
and return intensity) improved measurement precision and LDL over the baseline WMS case by approximately $41 \%$ and $40 \%$ respectively. In addition, estimating the absorptionfree intensity using the concurrent intensity correction approach further improved precision by approximately $72 \%$ (to $1.8 \mathrm{ppm}_{\mathrm{v}}$ ) and the LDL by $73 \%$ (to $1.7 \mathrm{ppm}_{\mathrm{V}}$ ). Finally, by including pair-wise averaging of the leading and lagging $2 f$ values the precision and LDL were improved by an additional $9 \%$ (to $1.62 \mathrm{ppm}_{\mathrm{v}}, 6.57 \times 10^{-7} \mathrm{~cm}^{-1}$ in equivalent absorbance) and $10 \%$ (to $1.51 \mathrm{ppm}_{\mathrm{v}}, 6.13 \times 10^{-7} \mathrm{~cm}^{-1}$ in equivalent absorbance) respectively. Alternatively, when the intensity-corrected experimental calibration method was used (combined with the concurrent intensity correction and pair-wise averaging approaches), the measured methane VMR precision was not as good at $1.9 \mathrm{ppm}_{\mathrm{V}}$ $\left(7.72 \times 10^{-7} \mathrm{~cm}^{-1}\right)$, but resulted in a lower LDL value at $1.36 \mathrm{ppm}_{\mathrm{v}}\left(5.50 \times 10^{-7} \mathrm{~cm}^{-1}\right)$.

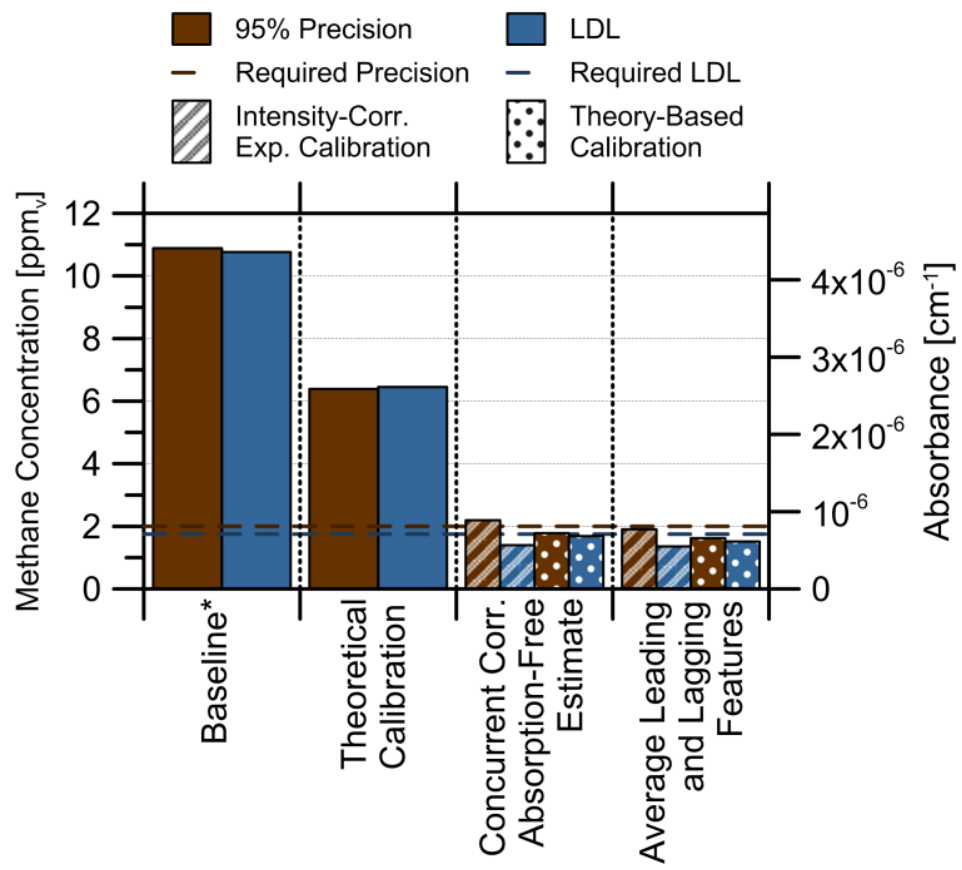

Figure 5.19: Measurement performance improvements for a WMS-based system for various hardware and software system enhancements, for the $2 v_{3} R(3)$ methane manifold at ambient temperature and pressure. 


\subsubsection{Evaluation of Long-term Drift}

Long-term implementation of a fiber coupled open-path optical sensor in the field presents significant albeit surmountable challenges (Stewart et al., 2010). Firstly, contamination of the laser beam-steering surfaces can be minimized by enclosing them within a dust and moisture resistant enclosure, and passing the laser beam through angled windows coated to ensure high transmittance and resistance to scratches, moisture, and dust buildup. Aside from requiring routine cleaning, signal loss due to window contamination is inherently included in the intensity-normalized calibration, and will lead to an increase in the measurement precision values proportional to the decrease in the SNR of the measured $2 f$ value.

In addition to the stability of the sensors and optical network, the long-term stability of the system performance was assessed using datasets acquired 4, 12, and up to 16-months after the initial calibration. Figure 5.20 and Figure 5.21 illustrate the measured precision and drift of the mean values for the SI and WMS methods (calculated as the absolute difference between the measured and requested methane VMR) for methane in air concentrations of $2.0 \mathrm{ppm}_{\mathrm{v}}$ and $50 \mathrm{ppm}_{\mathrm{v}}$, with the $2 v_{3} \mathrm{R}(3)$ methane manifold around atmospheric temperature and pressure. 


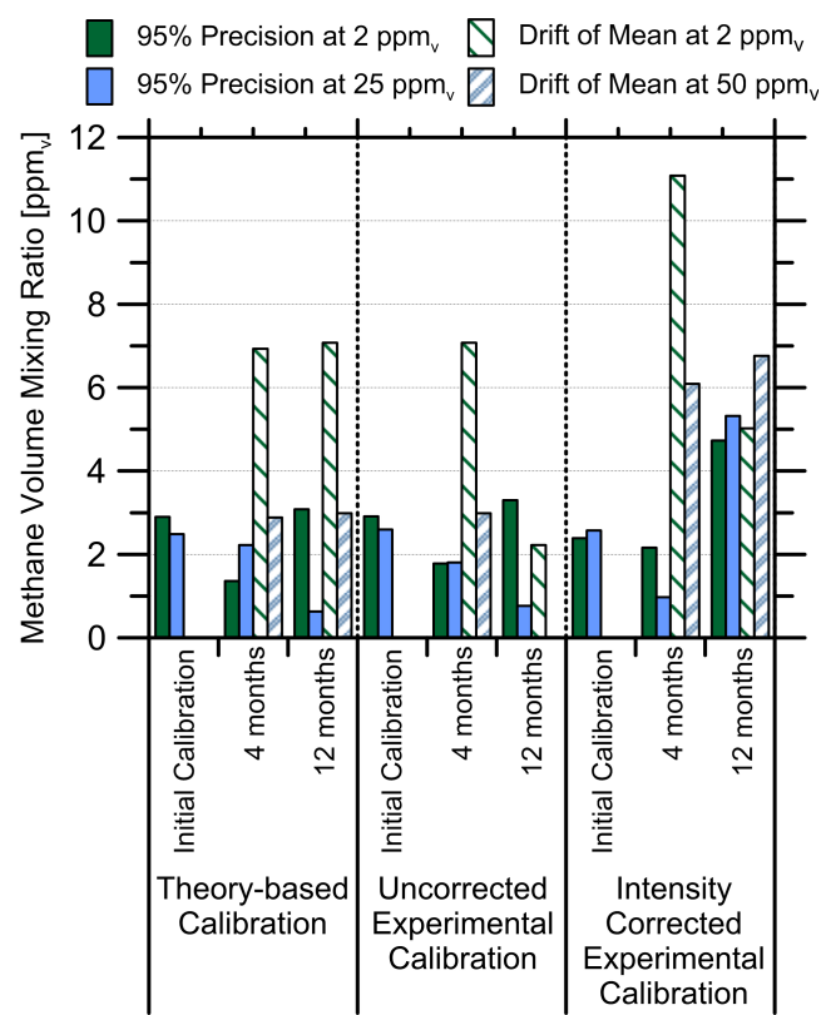

Figure 5.20: Long-term system methane volume mixing ratio measurement precision and mean drift for the SI modulation scheme, using the concurrent method for estimating absorption-free intensity, for the $2 v_{3} \mathrm{R}(3)$ methane manifold at ambient temperature and pressure. 


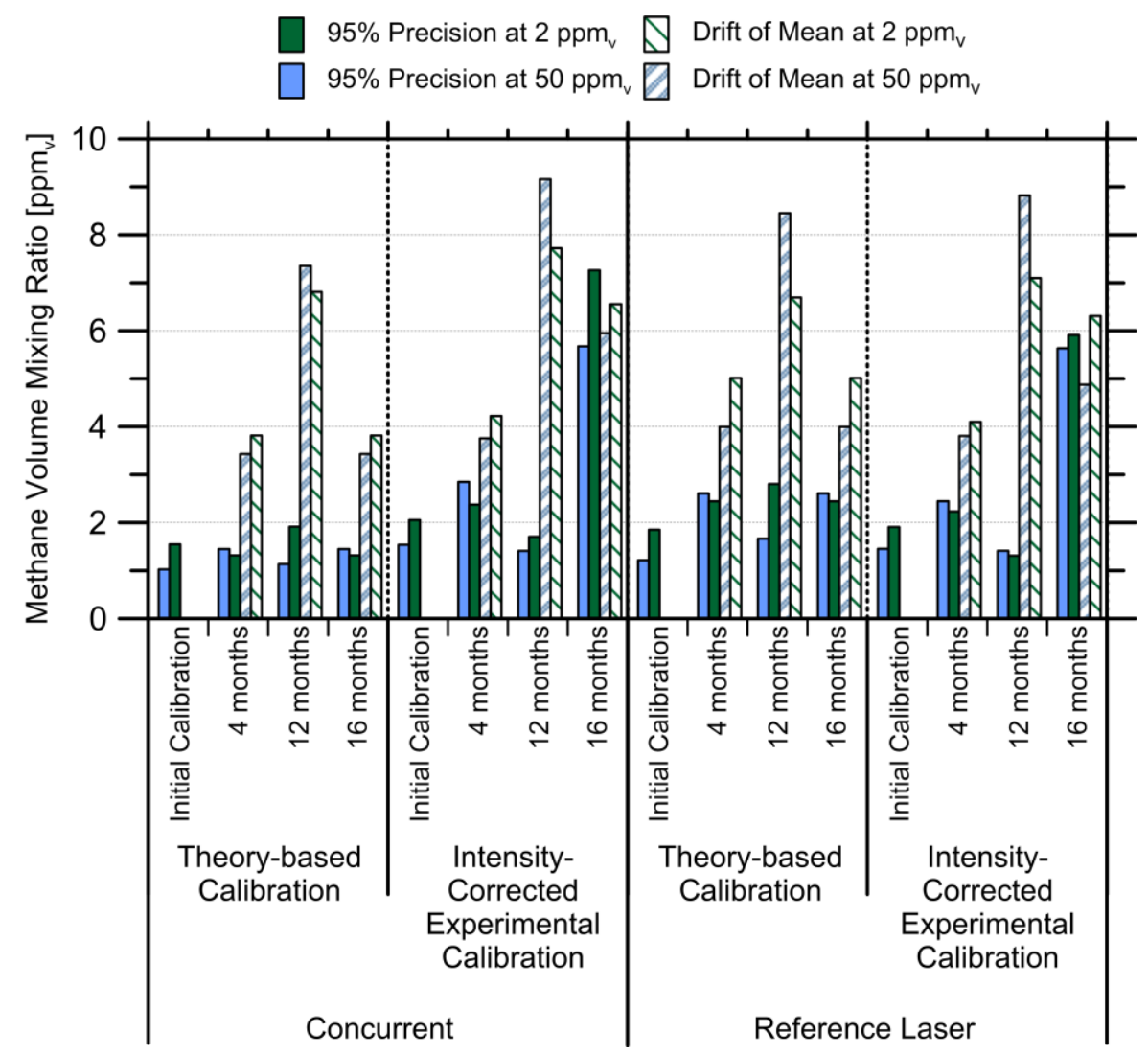

Figure 5.21: Long-term system methane volume mixing ratio measurement precision and mean drift for the WMS modulation scheme, using the concurrent and reference laser methods for estimating absorption-free intensity, for the $2 v_{3} \mathbf{R}(3)$ methane manifold at ambient temperature and pressure.

These results illustrate a minimal variation in measurement precision for the SI and WMS modulation schemes over the tested timeframes. However, over the course of a year, drift in the mean measured methane volume mixing ratio was observed. This drift reached $11.1 \mathrm{ppm}_{\mathrm{v}}$ for the SI method implementing the intensity-corrected experimental calibration, and $7.35 \mathrm{ppm}_{\mathrm{v}}$ for the WMS methods implementing a theory-based calibration combined with concurrent intensity correction methods. The drift of the mean values may be attributable to network fiber replacement between the optical sensor head and base TDLAS system, as well as the cleaning and re-alignment of the reflective surfaces within the optical sensor head. Given that the latter activities would most likely occur during field maintenance, and given that there is also potential for fiber breakage and replacement in an industrial setting, the ambient methane VMR (zero-response) would need to be established regularly to minimize the accumulation drift errors. 
Simulations suggest (Brereton and Johnson, 2012) that an ambient methane VMR estimation should be achievable with software analysis, due to the infrequent interaction of fugitive emission plumes with sensors in a distributed network.

\subsubsection{Final Results and Comparison to Literature}

Table 5.6 compares the achieved WMS results to the target atmospheric methane VMR LDL of $\sim 1.7 \mathrm{ppm}_{\mathrm{v}}$ (or $\sim 6.89 \times 10^{-7} \mathrm{~cm}^{-1}$ ) and measurement precision of $2.0 \mathrm{ppm}_{\mathrm{v}}$ (or $\left.8.10 \times 10^{-7} \mathrm{~cm}^{-1}\right)$, the target set for the purpose of locating fugitive methane sources using trajectory statistical methods (Brereton and Johnson, 2012).

Table 5.6: Performance Characteristics of Fiber-Based TDLAS Systems Including Measurement Results for this System

\begin{tabular}{|c|c|c|c|c|c|}
\hline Reference & $\begin{array}{l}\text { Detection } \\
\text { Technique }^{\dagger}\end{array}$ & $\begin{array}{l}\text { Conditions of } \\
\text { Validation }^{\ddagger}\end{array}$ & $\begin{array}{c}\text { Wavelength of } \\
\text { Calculated*** } \\
\text { Absorption Feature } \\
\text { Maximum* [nm] }\end{array}$ & $\begin{array}{l}\text { VMR Standard } \\
\text { Deviation** } \\
(2 \sigma)\left[p_{\mathrm{ppm}}\right]\end{array}$ & $\begin{array}{l}\text { Absorbance } \\
\text { Standard } \\
\text { Deviation*** } \\
(2 \sigma)\left[\mathrm{cm}^{-1}\right]\end{array}$ \\
\hline He et al. (2014) & CRDS & $9 \mathrm{ppm}_{\mathrm{v}} \mathrm{NH}_{3}$ in $\mathrm{N}_{2}$ & 1531.682 & 0.0216 & $1.1 \times 10^{-8}$ \\
\hline Cao et al. (2013) & $2 f-\mathrm{PA}$ & $1 \% \mathrm{C}_{2} \mathrm{H}_{2}$ in $\mathrm{N}_{2}$ & 1555.836 & 4.3 & $1.3 \times 10^{-8}$ \\
\hline Current System & $2 f-W M S$ & $2 \mathrm{ppm}_{\mathrm{v}} \mathrm{CH}_{4}$ in Air & 1653.730 & 1.62 & $6.5 \times 10^{-7}$ \\
\hline LDL Target & & & & & $6.9 \times 10^{-7}$ \\
\hline Precision Target & & & & & $8.1 \times 10^{-7}$ \\
\hline Tai et al. (1992) & $2 f-W M S$ & $350 \mathrm{ppm}_{\mathrm{v}} \mathrm{CH}_{4}$ in Air & 1665.962 & $5^{b}$ & $4.0 \times 10^{-6}$ \\
\hline Bauer et al. (2014) & $2 f-\mathrm{PA}$ & $100 \mathrm{ppm}_{\mathrm{v}} \mathrm{C}_{2} \mathrm{H}_{2}$ in $\mathrm{N}_{2}$ & 1532.831 & 2.5 & $5.3 \times 10^{-6}$ \\
\hline Stewart et al. (2010) & $2 f-W M S$ & $25 \mathrm{ppm}_{\mathrm{v}} \mathrm{CH}_{4}$ in Air & 1650.959 & $50^{c}$ & $4.8 \times 10^{-5}$ \\
\hline Zhang et al. (2012) & $\mathrm{DA}$ & $1.0 \% \mathrm{CH}_{4}$ in Air & 1645.543 & 100 & $9.9 \times 10^{-5}$ \\
\hline Yu et al. (2001) & $2 f-W M S$ & $1.0 \% \mathrm{C}_{2} \mathrm{H}_{2}$ in Air & 1530.371 & 165 & $3.9 \times 10^{-4}$ \\
\hline Ho et al. (2000) & $2 f-W M S$ & $0.94 \% \mathrm{C}_{2} \mathrm{H}_{2}$ in Air & 1530.371 & $270^{b}$ & $6.3 \times 10^{-4}$ \\
\hline Li (2006) & $2 f-W M S$ & $790 \mathrm{ppm}_{\mathrm{v}} \mathrm{CH}_{4}$ in Air & 1645.543 & $750^{b}$ & $7.4 \times 10^{-4}$ \\
\hline Chan et al. (1985) & DA & $1 \% \mathrm{CH}_{4}$ in Air & 1665.962 & $1315.8^{a}$ & $1.0 \times 10^{-3}$ \\
\hline \multicolumn{6}{|c|}{$\begin{array}{l}\dagger \text { CRDS - Cavity Ring-Down Spectroscopy, DA - Direct Absorption, 2f-WMS Second-harmonic Wavelength Modulation } \\
\text { Spectroscopy, PA-Photo-acoustic }\end{array}$} \\
\hline
\end{tabular}

When compared in terms of an instrument-dependant absorbance, the precision and LDL achieved by this system were lower than published results from comparable alloptical fiber-coupled $2 f$-WMS systems implementing all-optical remote sensors, $4.8 \times 10^{-5} \mathrm{~cm}^{-1}$ by (Stewart et al., 2010) and $4.0 \times 10^{-5} \mathrm{~cm}^{-1}$ by (Tai et al., 1992), and were 
comparable to the performance of an all-optical fiber-coupled photo-acoustic based system for the detection of acetylene which achieved a precision of $5.3 \times 10^{-6} \mathrm{~cm}^{-1}$ (Bauer et al., 2014).

The precision achieved by He et al. (2014) and Cao et al. (2013) using CRDS and $2 f$-PA systems respectively is over 50 times lower than what was achieved by this TDLAS $2 f$-WMS system. However, as previously stated in Chapter 2, these systems may not be particularly suited for the application of detecting ambient methane in an industrial environment due to sensor head fragility and lack of an all-optical solution. 


\section{Chapter 6 Conclusions}

A fiber-optically networked tunable diode laser absorption spectroscopy system was developed for a target application of in situ, multipoint monitoring of ambient methane within an industrial environment such as an oil and gas processing plant. The system was designed to fulfil accuracy, sensitivity, and response-time requirements of a separate modelling scheme to enable continuous location and quantification of unknown fugitive leak sources.

The requirement to detect low concentrations of methane at ambient pressures and temperatures using an array of sensors governed the design of the optical sensor heads. To balance the trade-offs between SNR and optical coupling stability, the custom fibercoupled open-path optical sensor head used in this system employed an arrangement of planar reflective surfaces to route the laser beam through an open interrogation region, achieving a total optical path-length of $5.79 \mathrm{~m}$ in 8 passes.

The base-system utilized fiber-coupled optical components and was designed to quickly switch between the sweep integration (SI) and wavelength modulation spectroscopy (WMS) methods to directly compare methane volume mixing ratio (VMR) measurement performance without reconfiguring hardware. Optimum laser diode driving signals were selected (within the capabilities of the signal generators, laser controllers, and data acquisition hardware) through parametric experiments to maximize the SI absorption and WMS second-harmonic (2f) feature SNR, the change in measured absorption and $2 f$ with change in methane VMR, and the absolute difference between the theory-based and measured absorption and $2 f$ features. Systematic experiments were subsequently performed to quantitatively evaluate the effects of various signal processing and hardware modifications on system drift, lower detection limit, and measurement precision. 
Measurement drift was the most significant limiter of system performance, and the largest contributor to this drift was the thermal stability of the fiber optical components within the base-system. Thermal stabilization and polarization scrambling were both investigated as means of supressing drift effects. Although both approaches offered significant improvements, thermal stabilization alone achieved the best performance results. Further reduction of system drift effects required implementation of methods to accurately estimate the time-varying absorption-free intensity. An automated daily system-calibration was implemented to determine varying laser characteristics and relationships between the absorption-free intensities over multiple photodetectors and laser diodes. In addition, concurrent intensity corrections were investigated including software derivation of the absorption-free intensity from the mean post-absorption intensity as well as the use of a second reference laser (spectrally located near the absorption laser, in a region free of absorption from typical atmospheric gases). Of these, the software-based approach was shown to be the most effective.

The influence of residual amplitude modulation (RAM) of the laser diode intensity was observed in the consistent discrepancy in the magnitude of the absorption or $2 f$ features obtained when the absorption manifold was traversed with an increasing or decreasing wavelength. Mitigation of RAM effects were investigated via a direct signal subtraction technique in post-processing and via pair-wise averaging of the absorption or $2 f$ feature maxima values obtained as wavelength was increasing and decreasing during each sweep period. The latter of these approaches was shown to be most effective.

In this system the volume mixing ratio was derived from measured absorption signals (SI) or $2 f$ signals (WMS) using either a theory-based or experimental calibration. The theory-based calibration relied on spectroscopic theory to calculate predicted methane concentrations from measured absorption or $2 f$ values, estimated absorption-free intensity signals, and ambient temperature and pressure values. This theory-based predicted methane VMR was then related to actual methane VMR via an empirical calibration (which accounted for uncertainties in the spectroscopic theory and available absorption line data) determined via least squares regression to experimental data with known volume mixing ratios. The experimental calibration simply estimated methane 
VMR data directly from measured absorption or $2 f$ values and estimated absorption-free intensity signals via an empirical calibration derived from calibration experiments with known volume mixing ratios. The resultant theory-based and empirical calibration functions and consequent best-fit prediction intervals were used to quantify methane VMR measurement precision, drift, and LDL.

Overall, the best system performance for both the SI and WMS methods was achieved using thermally stabilized optical components without polarization scrambling, automated daily calibration supported by concurrent software-based estimation of the absorption-free intensity, pair-wise averaging of the absorption or $2 f$ feature maxima in each sweep period to suppress effects of RAM. The SI method achieved the best measurement results when the intensity-corrected experimental calibration technique was used, while the WMS method achieved peak performance using a theory-based calibration. The best measurement results from the SI technique were not able to match the performance of the WMS technique, reaching a minimum methane VMR measurement precision of $3.14 \mathrm{ppm}_{\mathrm{v}}$ and LDL of $1.07 \mathrm{ppm}_{\mathrm{v}}$ (equivalent to an absorbance precision of $1.27 \times 10^{-6} \mathrm{~cm}^{-1}$ and LDL of $4.35 \times 10^{-7} \mathrm{~cm}^{-1}$ ). The WMS technique achieved a methane measurement precision of $1.62 \mathrm{ppm}_{\mathrm{v}}$ (absorbance precision of $6.57 \times 10^{-7} \mathrm{~cm}^{-1}$ ) and LDL $1.51 \mathrm{ppm}_{\mathrm{v}}\left(6.13 \times 10^{-7} \mathrm{~cm}^{-1}\right)$, which, notably, was better than the target precision of $2.0 \mathrm{ppm}_{\mathrm{v}}$ (or $8.10 \times 10^{-7} \mathrm{~cm}^{-1}$ ) and LDL of $\sim 1.7 \mathrm{ppm}_{\mathrm{v}}\left(\right.$ or $\sim 6.89 \times 10^{-7} \mathrm{~cm}^{-1}$ ) required to locate fugitive methane sources using statistical trajectory methods (Brereton and Johnson, 2012).

By surpassing relevant precision and LDL targets for quantifying fugitive methane plumes at near ambient concentrations, the fiber optically networked TDLAS system presented here has demonstrated potential for use in the detection of fugitive emissions. While there are systems in the literature that have achieved lower measurement precision values, they have done so using hardware and techniques which may not be well suited for the target application which requires intrinsically safe, remote, in situ, multipoint measurement of atmospheric methane in an industrial environment. Furthermore, when the achieved WMS measurement results are stated in terms of an instrument-dependant absorbance, the measured precision $\left(6.57 \times 10^{-7} \mathrm{~cm}^{-1}\right)$ and LDL 
$\left(6.13 \times 10^{-7} \mathrm{~cm}^{-1}\right)$ values are lower than published results achieved with the few other comparable fiber-coupled $2 f$-WMS systems implementing all-optical remote sensors (Tai et al., 1992; Stewart et al., 2010).

\subsection{Future Considerations}

\subsubsection{Interrogation Cell Changes}

The fiber-coupled methane sensing head in the present system used surface-reflecting 'hollow' reflectors to route the beam through the interrogation volume. Losses due to optical scattering and mechanical misalignment were minimized with the use of high quality surfaces and monolithic reflectors where possible (Chapter 3). Although the performance of this inexpensive optical sensor prototype has been demonstrated in laboratory conditions, the assembled sensor head is large, measuring approximately 70 by 20 by $20 \mathrm{~cm}$. This is larger than would likely be preferred for field-deployment, and the overall dimensions are driven by the sizes of commercially available reflecting elements and the optical path-length required for the sensitive measurement of methane near atmospheric concentrations.

A refined multipass fiber-coupled sensor head has been preliminarily designed that could meet or exceed the current methane measurement performance while also theoretically improving sensor cost, size, manufacturability, and stability. Sensor size could be decreased by increasing the number of interrogation-region passes to reduce overall sensor-head size. However, the trade-offs of such a sensor-head are not clear, and experiments and cost-studies would need to be performed to properly size and select the reflecting elements. Initial estimates indicate that if antireflective coated prisms can be used to achieve an optical path length of $5.8 \mathrm{~m}$ in 12 passes (instead of 8), this new sensor could have a lower estimated manufacturing cost (reducing the overall cost of a system implementing many sensor heads over an industrial facility) and significantly reduced overall dimensions (from $\sim 70 \times 20 \times 20 \mathrm{~cm}$ to $\sim 55 \times 5 \times 5 \mathrm{~cm}$ ). 


\subsubsection{Network Changes}

The demonstrated TDLAS system employs the most technically straightforward fiber optical multiplexing method, i.e. spatial multiplexing via a selectable fiber optic switch (Section 3.1.3). However, the WMS configuration of this system was also designed to permit incorporation of other multiplexing techniques as the application requires. For example, if simultaneous detection of multiple gas species were required, the spectral absorption of multiple molecules could be observed independently by way of optical wavelength-multiplexing and/or modulation frequency multiplexing with very few system modifications. Using the former technique multiple lasers (with sufficient spectral separation) could be coupled into the system to simultaneously observe multiple absorption manifolds, with return signals being split onto multiple photodetectors using spectral gratings. The latter technique would utilize two lasers with similar wavelengths, each modulated at different carrier frequencies, and detected by a single photodetector. The harmonic signals for each laser could then be separated from the resultant combined photodetector signal by demodulating at their respective carrier frequencies. Use of these methods could permit the simultaneous quantification of various gas characteristics such as VMR, pressure, temperature, and bulk-flow velocity for multiple gas species. Similar systems have been constructed for the study of combustion products with promising success (Rieker et al., 2007; Rieker, 2009). By combining these various multiplexing methods, system hardware could be used for more than one purpose, adding overall functionality without drastically increasing hardware cost.

\subsubsection{Amplitude Modulation Attenuation}

This TDLAS system implemented pair-wise averaging of the leading and lagging absorption or $2 f$ feature peak-values to supress the influence of RAM on the measured methane VMR. While this approach was very successful in compensating for RAM, it would not necessarily be applicable in systems implementing the wavelength multiplexing method. For this reason, other methods of RAM suppression would need to be considered. One approach that has been preliminarily investigated is the use of a highspeed variable optical attenuator (VOA) and a field-programmable gate array (FPGA) to 
attenuate the modulated laser output signal in real-time to achieve a RAM-free intensity signal. It is recommended that this approach be more thoroughly investigated in the future.

\subsubsection{Outdoor Testing}

Based on the demonstrated capabilities of the current lab-based prototype, long-term installation of a full field deployable system implementing multiple sensor heads in an exposed outdoor environment is the next logical step in quantifying the measurement performance of this system. Specifically, investigation in to the influence of reflective surface degradation (due to dust, grime, condensation, and scratches), and large temperature fluctuations is paramount. Finally, a comparison of simulated and measured results from a controlled methane-plume release experiment would be invaluable in demonstrating the viability of this system to quantify and locate fugitive methane plume sources in an industrial environment. 


\section{References}

Altmann, J., Baumgart, R., and Weitkamp, D.C., (1981). Two-mirror multipass absorption cell. Appl. Opt. 20:995-999.

Ameer, Q. and Adeloju, S.B., (2005). Polypyrrole-based electronic noses for environmental and industrial analysis. Sensors Actuators B Chem.. 106:541-552.

Andersson, M., Persson, L., Svensson, T., and Svanberg, S., (2007). Flexible lock-in detection system based on synchronized computer plug-in boards applied in sensitive gas spectroscopy. Rev. Sci. Instrum.. 78:1-7.

Bacharach Inc., (2002). HI FLOW Sampler: Technical Specifications. http://www.bacharach-inc.com/PDF/Brochures/4159 Hi Flow.pdf

Baer, D.S., Hanson, R.K., Newfield, M.E., and Gopaul, N.K., (1994). Multiplexed diodelaser sensor system for simultaneous $\mathrm{H} 2 \mathrm{O}, \mathrm{O} 2$, and temperature measurements. Opt. Lett.. 19:1900.

Baer, D.S., Nagali, V., Furlong, E.R., Hanson, R.K., and Newfield, M.E., (1996). Scanned- and fixed-wavelength absorption diagnostics for combustion measurements using multiplexed diode lasers. AIAA J.. 34:489-493.

Bauer, R., Stewart, G., Johnstone, W., Boyd, E., and Lengden, M., (2014). 3D-printed miniature gas cell for photoacoustic spectroscopy of trace gases. Opt. Lett.. 39:4796-9.

Berden, G., Peeters, R., and Meijer, G., (2000). Cavity ring-down spectroscopy: experimental schemes and applications, Int. Rev. Phys. Chem. 19:565-670.

Berden, G., Peeters, R., and Meijer, G., (2000). Cavity ring-down spectroscopy: experimental schemes and applications. Rev. Phys. Chem.. 19:565-670.

Berman, E.S.F., Fladeland, M., Liem, J., Kolyer, R., and Gupta, M., (2012). Greenhouse gas analyzer for measurements of carbon dioxide, methane, and water vapor aboard an unmanned aerial vehicle. Sensors Actuators B Chem.. 169:128-135.

Bomse, D., Stanton, A., and Silver, J., (1992). Frequency modulation and wavelength modulation spectroscopies: Comparison of experimental methods using a lead-salt diode laser. Appl. Opt. 31:718-731.

Brahimi, H., Lacroix, P., and Llopis, O., (2009). Optimisation of a microwave frequency discriminator based on an optical delay line. In Microwave Photonics. Valencia, IEEE, pp.1-4.

Brereton, C.A. and Johnson, M.R., (2012). Identifying sources of fugitive emissions in industrial facilities using trajectory statistical methods. Atmos. Environ. 51:4655. 
Brumfield, B.E., Taubman, M.S., Suter, J.D., and Phillips, M.C., (2015). Characterization of a swept external cavity quantum cascade laser for rapid broadband spectroscopy and sensing. Opt. Express. 23:25553.

Cao, Y., Jin, W., Ho, H.L., and Ma, J., (2013). Miniature fiber-tip photoacoustic spectrometer for trace gas detection. Opt. Lett. 38:434-436.

CAPP, (2007). Best Management Practice: Management of Fugitive Emissions at Upstream Oil and Gas Facilities. 2007-0003, Calgary, AB, Canadian Association of Petroleum Producers (CAPP). Available at: http://www.capp.ca/getdoc.aspx?DocId=116116\&DT=PDF

Carlisle, C.B., Cooper, D.E., and Preier, H., (1989). Quantum noise-limited FM spectroscopy with a lead-salt diode laser. Appl. Opt.. 28:2567-76.

Carras, J.N., Franklin, P.M., Hu, Y., Singh, A.K., Tailakov, O. V., Picard, D., Azhari, F.M.A., Gjerald, E., Nordrum, S., and Yesserkepova, I., (2006). Chapter 4: Fugtive Emissions. In S. Eggleston, L. Buendia, K. Miwa, T. Mgara, \& K. Tanabe, eds. 2006 IPCC Guildelines for National Greenhouse Gas Inventories, Volume 2: Energy. Hayama Japan, Intergovernmental Panel on Climate Change (IPCC), pp.4.1-4.78.

Cassidy, D.T. and Bonnell, L.J., (1988). Trace gas detection with short-external-cavity InGaAsP diode laser transmitter modules operating at 1.58 microm. Appl. Opt. . 27:2688-93.

Cassidy, D.T. and Reid, J., (1982a). High-sensitivity detection of trace gases using sweep integration and tunable diode lasers. Appl. Opt.. 21:2527-2530.

Cassidy, D.T. and Reid, J., (1982b). Atmospheric pressure monitoring of trace gases using tunable diode lasers. Appl. Opt. 21. 21:1185-1190.

Chakraborty, A.L., Ruxton, K., and Johnstone, W., (2010). Influence of the wavelengthdependence of fiber couplers on the background signal in wavelength modulation spectroscopy with RAM-nulling. Opt. Express. 18:3114-3125.

Chambers, A.K., Strosher, M., Wootton, T., Moncrieff, J., and McCready, P., (2008). Direct measurement of fugitive emissions of hydrocarbons from a refinery. J. Air Waste Manag. Assoc. 58:1047-56.

Chambers, P., Austin, E.A.D., and Dakin, J.P., (2004). Theoretical analysis of methane gas detection system, using the complementary source modulation method of correlation spectroscopy. Meas. Sci. Tech. 15:1629-1636.

Chan, K., Ito, H., and Inaba, H., (1984). An Optical-Fiber-Based Gas Sensor for Remote Absorption Measurement of Low-Level CH4 Gas in the Near-Infrared Region. $J$. Light. Technol.. LT-2:234-237.

Chan, K., Ito, H., and Inaba, H., (1984). Remote sensing system for near-infrared differential absorption of $\mathrm{CH}_{-} 4$ gas using low-loss optical fiber link. Appl. Opt.. 23:3415. 
Chan, K., Ito, H., Inaba, H., and Furuya, T., (1985). 10 km-long fibre-optic remote sensing of $\mathrm{CH} 4$ gas by near infrared absorption. Appl. Phys. B. 38:11-15.

Chan, P.K.C., Jin, W., Member, S., and Demokan, M.S., (2000). FMCW Multiplexing of Fiber Bragg Grating Sensors. Quantum. 6:756-763.

Chavali, M., Lin, T., Wu, R., Luk, H., and Hung, S., (2008). Active 433 MHz-W UHF RF-powered chip integrated with a nanocomposite m-MWCNT / polypyrrole sensor for wireless monitoring of volatile anesthetic agent sevoflurane. Sensors And Actuators. 141:109-119.

Chernin, S., (2001). Development of optical multipass matrix systems. J. Mod. Opt. . 48:619-632.

Collett, E., (2003). Polarized Light in Fiber Optics. Bellingham, SPIE Press.

Culshaw, B., (2004). Optical Fiber Sensor Technologies: Opportunities and-PerhapsPitfalls. J. Light. Technol.. 22:39-50.

Cvijin, P.V., Wells, W.K., Gilmore, D.A., Wu, J., Hunten, D.M., and Atkinson, G.H., (1992). Fringe pattern suppression in intracavity laser spectroscopy. Appl. Opt. . $31: 3-8$.

D’Amato, F., (2006). Laser Spectrometers for Atmospheric Analysis B. D. Bartolo \& O. Forte, eds. Springer.

D’Amato, F., Mazzinghi, P., and Castagnoli, F., (2002). Methane analyzer based on TDL's for measurements in the lower stratosphere: design and laboratory tests. Appl. Phys. B Lasers Opt. 75:195-202.

Dahnke, H., Kleine, D., Hering, P., and Mürtz, M., (2001). Rapid communication Realtime monitoring of ethane in human breath using mid-infrared cavity leak-out spectroscopy. . 975:971-975.

Dakin, J.P., (1987). Multiplexed and distributed optical fibre sensor systems. J. Phys. E.. 20:954-967.

De Tommasi, E., Casa, G., and Gianfrani, L., (2007). An Intensity-Stabilized DiodeLaser Spectrometer for Sensitive Detection of NH3. IEEE Trans. Instrum. Meas.. 56:309-312.

Disselkamp, R.S., Kelly, J.F., Sams, R.L., and Anderson, G.A., (2002). Signal-to-noise enhancement techniques for quantum cascade absorption spectrometers employing optimal filtering and other approaches. Appl. Phys. B Lasers Opt.. 75:359-366.

Dlugokencky, E.J., Nisbet, E.G., Fisher, R., and Lowry, D., (2011). Global atmospheric methane: budget, changes and dangers. Philos. Trans. A. Math. Phys. Eng. Sci.. 369:2058-72.

Dong, L., Yin, W., Ma, W., Zhang, L., and Jia, S., (2007). High-sensitivity, large dynamic range, auto-calibration methane optical sensor using a short confocal Fabry-Perot cavity. Sensors Actuators B Chem.. 127:350-357. 
Dubaniewicz, T.H., (2006). Methane-air mixtures ignited by CW laser-heated targets on optical fiber tips: Comparison of targets, optical fibers, and ignition delays. $J$.

Loss Prev. Process Ind. . 19:425-432.

Duffin, K., McGettrick, A.J., Johnstone, W., Stewart, G., and Moodie, D.G., (2007).

Tunable Diode-Laser Spectroscopy With Wavelength Modulation: A CalibrationFree Approach to the Recovery of Absolute Gas Absorption Line Shapes. $J$. Light. Technol.. 25:3114-3125.

Durry, G. and Pouchet, I., (2002). Open multipass absorption cell for in situ monitoring of stratospheric trace gas with telecommunication laser diodes. Appl. Opt. . 41:424-433.

Edwards, H.O. and Dakin, J.P., (1993). Gas sensors using correlation spectroscopy compatible with fibre optic operation.pdf. Sensors Actuators B Chem.. 11:9 - 19.

Engeln, R., Helden, G. Von, Berden, G., and Meijer, G., (1996). Phase shift cavity ring down absorption spectroscopy. Chem. Phys.. 2614.

Environment Canada, (2014). National Inventory Report. Ottawa, ON.

EPA, (1995). Protocol for Equipment Leak Emission Estimates (EPA Publication No. 453-R-95-017). Triangle Park, NC: U.S.A.

Faglia, G., Comini, E., Pardo, M., Taroni, A., Cardinali, G., Nicoletti, S., and Sberveglieri, G., (1999). Micromachined gas sensors for environmental pollutants. Microsyst. Technol.. 6:54-59.

Fernholz, T., Teichert, H., and Ebert, V., (2002). Digital, phase-sensitive detection for in situ diode-laser spectroscopy under rapidly changing transmission conditions. Appl. Phys. B Lasers Opt. 75:229-236.

Fischer, J., (2003). Total internal partition sums for molecular species in the 2000 edition of the HITRAN database. J. Quant. Spectrosc. Radiat. Transf.. 82:401-412.

Fox, R.W., Oates, C.W., and Hollberg, L.W., (2002). Stabilizing Diode Lasers to HighFinesse Cavities. In R. Van Zee \& J. Looney, eds. Cavity-Enhanced Spectroscopies. Boulder, Colorado, National Institude of Standards and Technology, pp.1-46.

Gardiner, T., Mead, M.I., Garcelon, S., Robinson, R., Swann, N., Hansford, G.M., Woods, P.T., and Jones, R.L., (2010). A lightweight near-infrared spectrometer for the detection of trace atmospheric species. Rev. Sci. Instrum. 81:083102.

Gas Measurement Instruments Ltd., (2014). GT Series Portable Detector: Technical Specifications. http://www.gmiuk.com/products/portable-detectors/gt-series

Gasmet, (2015). Multipoint Sampling Units. http:/www.gasmet.com/products/stationaryanalyzer-systems/multipoint-sampling-unit (accessed January 3, 2016)

Gehrtz, M., Bjorklund, G., and Whittaker, E., (1985). Quantum-limited laser frequencymodulation spectroscopy. Opt. Soc. Am. B.. 2:1510-1526. 
GMI, (2010). Global Methane Emissions and Mitigation Opportunities. Available at: https://www.globalmethane.org/documents/analysis_fs_en.pdf

Gordley, L.L., Marshall, B.T., and Allen Chu, D., (1994). Linepak: Algorithms for modeling spectral transmittance and radiance. J. Quant. Spectrosc. Radiat. Transf.. 52:563-580.

Guan, Z., Svanberg, K., Svensson, T., and Lewander, M., (2009). Clinical system for non-invasive in situ monitoring of gases in the human paranasal sinuses. Opt. Express. 17:345-354.

Guan, Z., Zhou, B., Liu, G., He, S., and Member, S., (2007). Quasi-Distributed Absorption Sensing System Based on a Coherent Multiplexing Technique. Technology. 19:792-794.

Gurlit, W., Zimmermann, R., Giesemann, C., Fernholz, T., Ebert, V., Wolfrum, J., Platt, U., and Burrows, J.P., (2005). Lightweight diode laser spectrometer CHILD (Compact High-altitude iN-situ Laser Diode) for balloonborne measurements of water vapor and methane. Appl. Opt.. 44:91-102.

Hastie, D.R. and Miller, M.D., (1985). Balloon-borne tunable diode laser absorption spectrometer for multispecies trace gas measurements in the stratosphere. Appl. Opt. 24:3694-01.

Hayward, J.E., Cassidy, D.T., and Reid, J., (1989). High-sensitivity transient spectroscopy using tunable diode lasers. Appl. Phys. B Photophysics Laser Chem.. 48:25-29.

He, Y., Jin, C., Kan, R., Liu, J., Liu, W., Hill, J., Jamie, I.M., and Orr, B.J., (2014). Remote open-path cavity-ringdown spectroscopic sensing of trace gases in air, based on distributed passive sensors linked by km-long optical fibers. Opt. Express. 22:13170.

He, Y., Kan, R., Englich, F. V, Liu, W., and Orr, B.J., (2010). level sensing of gas mixtures by rapidly swept continuous-wave cavity-ringdown spectroscopy. Opt. Express. 18:20059-20071.

Heath US, (2013). RMLD-IS: Technical Specifications. http://heathus.com/wpcontent/uploads/rmld-is_brochure.pdf

Hecht, E., (2002). Optics 4th ed. A. Black, ed. San Francisco, CA, Pearson Education Limited.

Heriott, D.R., Kogelnik, H., Kompfner, R., and Herriott, D.R., (1964). Off-axis paths in spherical mirror interferometers. Appl. Opt. 3:523-526.

Hinkley, E.D., (1970). High-Resolution Infrared Spectroscopy with a Tunable Diode Laser. Appl. Phys. Lett. 16:351-354.

Hinkley, E.D., Ku, R.T., Nill, K.W., and Butler, J.F., (1976). Long-path monitoring: advanced instrumentation with a tunable diode laser. Appl. Opt.. 15:1653-1655. 
Ho, H.L., Jin, W., Yu, H.B., Chan, K.C., Chan, C.C., and Demokan, M.S., (2000). Experimental demonstration of a fiber-optic gas sensor network addressed by FMCW. IEEE Photonics Technol. Lett. 12:1546-1548.

Hu, T.A., Chappell, E.L., Munley, J.T., and Sharpe, S.W., (1993). Improved multipass optics for diode-laser spectroscopy. Rev. Sci. Instrum.. 64:3380-3383.

Huang, H. and Lehmann, K.K., (2010). Long-term stability in continuous wave cavity ringdown spectroscopy experiments. Appl. Opt. 49:1378 - 1387.

Huang, J.-Q., Zheng, C.-T., Gao, Z.-L., Ye, W.-L., and Wang, Y.-D., (2014). NearInfrared Methane Detection Device Using Wavelength-Modulated Distributed Feedback Diode Laser around 1.654 $\mu \mathrm{m}$. Spectrosc. Lett.. 47:197-205.

Inaba, H., Kobayasi, T., Hirama, M., and Hamaza, M., (1979). Optical-fibre network system for air-pollution monitoring over a wide area by optical absorption method. Electron. Lett.. 15:749-751.

IPCC, (2007). Direct Global Warming Potentials. http://www.ipcc.ch/publications_and_data/ar4/wg1/en/ch2s2-10-2.html

Iseki, T., (2003). Calculation of the ratio between the second and first harmonic signals in wavelength-modulation spectroscopy for absorption measurement. Opt. Rev.. 10:24-30.

Iseki, T., (2004). A portable remote methane detector using an InGaAsP DFB laser. Environ. Geol.. 46:1064-1069.

Jennings, D.E., (1980). Absolute line strengths in $v 4,12 \mathrm{CH} 4$, a dual-beam diode laser spectrometer with sweep integration.pdf. Appl. Opt.. 19:2695-2700.

Jin, W., Cao, Y., Yang, F., and Ho, H.L., (2015). Ultra-sensitive all-fibre photothermal spectroscopy with large dynamic range. Nat. Commun.. 6:6767.

Jin, W., Stewart, G., Philp, W., Culshaw, B., and Demokan, M.S., (1997). Limitation of absorption-based fiber optic gas sensors by coherent reflections. Appl. Opt. 36:6251-6255.

Johnson, L.A., (2006). Laser Diode Burn-in and Reliability Testing. IEEE Communiations

Johnstone, W., McGettrick, A.J., Duffin, K., Cheung, A., and Stewart, G., (2008). Tunable Diode Laser Spectroscopy for Industrial Process Applications: System Characterization in Conventional and New Approaches. IEEE Sens. J.. 8:10791088 .

Joynes, I.M., (2013). Proof-of-Concept Inverse Micro-Scale Dipsersion Modelling for Fugitive Emissions Qantification in Industrial Facilities. Carleton University.

Joynes, I.M. and Johnson, M.R., (2014). Inverse Pollution Transport Modelling for Fugitive Emissions. In AWMA Annual Conference \& Exhibition. Long Beach, Air \& Waste Management Association. 
Kazemi, A.A., Mendoza, E., Goswami, K., and Kempen, L., (2013). Fiber optic oxygen sensor detection system for harsh environments of aerospace applications. In A. A. Kazemi, B. C. Kress, \& S. Thibault, eds. Proceedings of SPIE - The International Society for Optical Engineering. p.872002.

Khan, M. and Kang, S.-W., (2014). A High Sensitivity and Wide Dynamic Range FiberOptic Sensor for Low-Concentration VOC Gas Detection. Sensors. 14:2332123336.

Kirchgessner, D.A., Lott, R.A., Cowgill, R.M., Harrison, M.R., and Shires, T.M., (1997). Estimate of methane emissions from the U.S. natural gas industry. Chemosphere. 35:1365-90.

Kirschke, S., Bousquet, P., Ciais, P., Saunois, M., Canadell, J.G., Dlugokencky, E.J., Bergamaschi, P., Bergmann, D., Blake, D.R., Bruhwiler, L., et al., (2013). Three decades of global methane sources and sinks. Nat. Geosci.. 6:813-823.

Kluczynski, P. and Axner, O., (1999). Theoretical description based on Fourier analysis of wavelength-modulation spectrometry in terms of analytical and background signals. Appl. Opt. 38:5803-5815.

Kluczynski, P., Lindberg, Å.M., and Axner, O., (2001). spectrometry with frequencydoubled diode-laser. Appl. Opt. 40:783-793.

Kosterev, A., Wysocki, G., Bakhirkin, Y., So, S., Lewicki, R., Fraser, M., Tittel, F., and Curl, R.F., (2007). Application of quantum cascade lasers to trace gas analysis. Appl. Phys. B. 90:165-176.

Krzempek, K., Jahjah, M., Lewicki, R., Stefański, P., So, S., Thomazy, D., and Tittel, F.K., (2013). CW DFB RT diode laser-based sensor for trace-gas detection of ethane using a novel compact multipass gas absorption cell. Appl. Phys. B. 112:461-465.

LabView, (2009). Overview of Curve Fitting Models and Methods in LabVIEW. Austin, TX. Available at: http://www.ni.com/white-paper/6954/en/

Lackner, M., (2007). Tunable diode laser absorption spectroscopy ( TDLAS ) in the process industries- a review. Rev. Chem. Eng.. 2:65-147.

Laser Components, (2015a). Extended InGaAs Photodiodes. http://www.lasercomponents.com/fileadmin/user_upload/home/Datasheets/lcdgi/i g19-series.pdf (accessed July 1, 2015)

Laser Components, (2015b). Standard InGaAs Photodiodes. http://www.lasercomponents.com/fileadmin/user_upload/home/Datasheets/lcdgi/i g17-series.pdf (accessed July 1, 2015)

Lawrence, N.S., (2006). Analytical detection methodologies for methane and related hydrocarbons. Talanta. 69:385-392.

Leen, J.B., Yu, X.-Y., Gupta, M., Baer, D.S., Hubbe, J.M., Kluzek, C.D., Tomlinson, J.M., and Hubbell, M.R., (2013). Fast In Situ Airborne Measurement of Ammonia 
Using a Mid-Infrared Off-Axis ICOS Spectrometer. Environ. Sci. Technol. . 47:130823150605002.

Li, H., Rieker, G.B., Liu, X., Jeffries, J.B., and Hanson, R.K., (2006). Extension of wavelength-modulation spectroscopy to large modulation depth for diode laser absorption measurements in high-pressure gases. Appl. Opt.. 45:1052.

Li, S., (2006). Optical fiber gas sensor for remote detection of methane gas in coal mines. (Ph.D. Thesis). Stevens Institute of Technology.

Lindberg, M., U, O.A., and Kluczynski, P., (2001). Wavelength modulation absorption spectrometry - an extensive scrutiny of the generation of signals. At. Spectrosc.

Linnartz, H., (2004). A Remotely Controllable Optical Multi-Pass System. Phys. Scr. . $70: 1-2$.

Linnerud, I., Kaspersen, P., and Jaeger, T., (1998). Gas monitoring in the process industry using diode laser spectroscopy. Appl. Phys. B Lasers Opt. 67:297-305.

Liu, J.T.C., Jeffries, J.B., and Hanson, R.K., (2004a). Wavelength modulation absorption spectroscopy with $2 \mathrm{f}$ detection using multiplexed diode lasers for rapid temperature measurements in gaseous flows. Appl. Phys. B Lasers Opt. . 78:503511.

Liu, J.T.C., Jeffries, J.B., and Hanson, R.K., (2004b). Large-modulation-depth $2 \mathrm{f}$ spectroscopy with diode lasers for rapid temperature and species measurements in gases with blended and broadened spectra. Appl. Opt.. 43:6500-6509.

Liu, K., Wang, L., Tan, T., Wang, G., Zhang, W., Chen, W., and Gao, X., (2015). Highly sensitive detection of methane by near-infrared laser absorption spectroscopy using a compact dense-pattern multipass cell. Sensors Actuators B Chem.. 220:1000-1005.

Llopis, O., Azaizia, S., Saleh, K., Slimane, A.A., and Fernandez, A., (2013). Photodiode I/f noise and other types of less known baseband noises in optical telecommunications devices. In 2013 22nd International Conference on Noise and Fluctuations (ICNF). pp.1-4.

Loock, H.-P. and Wentzell, P.D., (2012). Detection limits of chemical sensors: Applications and misapplications. Sensors Actuators B Chem. 173:157-163.

Lyle, K., (2005). Development of a real-time diode-laser mass flux sensor for simultaneous measurement of density. (Ph.D. Thesis). Stanford University.

Manivannan, S., Saranya, A.M., Renganathan, B., Sastikumar, D., Gobi, G., and Park, K.C., (2012). Single-walled carbon nanotubes wrapped poly-methyl methacrylate fiber optic sensor for ammonia, ethanol and methanol vapors at room temperature. Sensors Actuators B Chem. 171-172:634-638.

Mappé, I., Joly, L., Durry, G., Thomas, X., Decarpenterie, T., Cousin, J., Dumelie, N., Roth, E., Chakir, A., and Grillon, P.G., (2013). A quantum cascade laser absorption spectrometer devoted to the in situ measurement of atmospheric N2O and CH4 emission fluxes. Rev. Sci. Instrum. 84:023103. 
Martan, T., Aubrecht, J., Podrazký, O., Matějec, V., and Kašík, I., (2014). Detection of hydrocarbons using suspended core microstructured optical fiber. Sensors Actuators B Chem. . 202:123-128.

Mohamed, T., Zhu, F., Chen, S., Strohaber, J., Kolomenskii, a a, Bengali, a a, and Schuessler, H. a, (2013). Multipass cell based on confocal mirrors for sensitive broadband laser spectroscopy in the near infrared. Appl. Opt.. 52:7145-51.

Moore, C.B., (1964). Applied Optics Letters to the Editor. Appl. Opt.. 4:252-253.

Myhre, G., Shindell, D., Bréon, F.-M., Collins, W., Fuglestvedt, J., Huang, J., Koch, D., Lamarque, J.-F., Lee, D., Mendoza, B., et al., (2013). Anthropogenic and Natural Radiative Forcing. D. Jacob, A. R. Ravishankara, \& K. P. Shine, eds., Cambridge, United Kingdom and New York, NY, USA, Cambridge University Press.

Nagali, V., Chou, S.I., Baer, D.S., Hanson, R.K., and Segall, J., (1996). Tunable diodelaser absorption measurements of methane at elevated temperatures. Appl. Opt.. 35:4026-32.

Nakaya, T., Kobayashi, K., Akagi, T., Kimura, K., and Tai, H., (2000). Continuous Monitoring of the Methane Concentration in the Atmosphere by IR Spectrometry with a 1 . 66- m Diode Laser. Anal. Chem.. 16:1211-1214.

Nwaboh, J.A., Hald, J., Lyngsø, J.K., Petersen, J.C., and Werhahn, O., (2013). Measurements of CO2 in a multipass cell and in a hollow-core photonic bandgap fiber at $2 \mu \mathrm{m}$. Appl. Phys. B. 110:187-194.

Olesberg, J.T., Liu, L., Van Zee, V., and Arnold, M. a, (2006). In vivo near-infrared spectroscopy of rat skin tissue with varying blood glucose levels. Anal. Chem.. 78:215-23.

Opekar, F. and Stulik, K., (2002). Amperometric Solid-State Gas Sensors: Materials for Their Active Components. Crit. Rev. Anal. Chem. 32:253-259.

Orr, B.J. and He, Y., (2011). Rapidly swept continuous-wave cavity-ringdown spectroscopy. Chem. Phys. Lett. 512:1-20.

Pal, S., Ozanyan, K.B., and McCann, H., (2008). A computational study of tomographic measurement of carbon monoxide at minor concentrations. Meas. Sci. Technol.. 19:094018.

Paldus, B. a and Kachanov, A. a, (2005). An historical overview of cavity-enhanced methods. Can. J. Phys. 83:975-999.

Pavone, F.S. and Inguscio, M., (1993). Frequency- and Wavelength-Modulation Spectroscopies: Comparison of Experimental Methods Using an AlGaAs Diode Laser. Appl. Phys. B. 56:118-122.

Persson, L., Andersson, F., Andersson, M., and Svanberg, S., (2007). Approach to optical interference fringes reduction in diode laser absorption spectroscopy. Appl. Phys. B. 87:523-530.

Phillips, M., (1992). Breath Tests in Medicine. Sci. Am.. 267:74-79. 
Picard, D., Stribrny, M., and Harrison, M.R., (1998). Handbook for Estimating Methane Emissions from Canadian Natural Gas Systems. Don Mills, ON.

Ragnauth, S., Petrusa, J., Alsalam, J., Godwin, D., Creason, J., Li, J., and Beach, R., (2013). Global Mitigation of Non-CO2 Greenhouse Gases.

Reid, J. and Labrie, D., (1981). Applied Physics Second-Harmonic Detection with Tunable Diode Lasers - Comparison of Experiment and Theory. Appl. Phys.. 210:203-210.

Ren, W., Jiang, W., and Tittel, F.K., (2014). Single-QCL-based absorption sensor for simultaneous trace-gas detection of CH4 and N2O. Appl. Phys. B. 117:245-251.

Richard, E.C., Kelly, K.K., Winkler, R.H., Wilson, R., Thompson, T.L., McLaughlin, R.J., Schmeltekopf, A.L., and Tuck, A.F., (2002). A fast-response near-infrared tunable diode laser absorption spectrometer for in situ measurements of $\mathrm{CH} 4$ in the upper troposphere and lower stratosphere. Appl. Phys. B Lasers Opt. . 75:183194.

Richter, D., Lancaster, D.G., and Tittel, F.K., (2000). Development of an automated diode-laser-based multicomponent gas sensor. Appl. Opt. 39:4444-50.

Rieker, G.B., (2009). WMS for measurements of gas temperature and concentration in harsh environments.

Rieker, G.B., Giorgetta, F.R., Swann, W.C., Kofler, J., Zolot, A.M., Sinclair, L.C., Baumann, E., Cromer, C., Petron, G., Sweeney, C., et al., (2014). Frequencycomb-based remote sensing of greenhouse gases over kilometer air paths. Optica. $1: 290$.

Rieker, G.B., Jeffries, J.B., and Hanson, R.K., (2009). Calibration-free wavelengthmodulation spectroscopy for measurements of gas temperature and concentration in harsh environments. Appl. Opt.. 48:5546-60.

Rieker, G.B., Li, H., Liu, X., Liu, J.T.C., Jeffries, J.B., Hanson, R.K., Allen, M.G., Wehe, S.D., Mulhall, P.A., and Kindle, H.S., (2007). Rapid measurements of temperature and $\mathrm{H} 2 \mathrm{O}$ concentration in IC engines with a spark plug-mounted diode laser sensor. Proc. Combust. Inst. . 31:3041-3049.

Riris, H., Carlisle, C.B., Warren, R.E., and Cooper, D.E., (1994). Signal-to-noise ratio enhancement in frequency-modulation spectrometers by digital signal processing. Opt. Lett. 19:144-146.

Roberson, B. and Cook, B., (2011). How to choose an Optical Power meter Detector. http://www.kingfisherfiber.com/Application-Notes/A19-How-Power-Meter.htm

Romanini, D., Kachanov, A., Sadenghi, N., and Stoeckel, F., (1997). CW cavity ring down spectroscopy. Chem. Phys.. 14.

Rothman, L.S., Gordon, I.E., Babikov, Y., Barbe, A., Chris Benner, D., Bernath, P.F., Birk, M., Bizzocchi, L., Boudon, V., Brown, L.R., et al., (2013). The HITRAN2012 molecular spectroscopic database. J. Quant. Spectrosc. Radiat. Transf.. 130:4-50. 
Rothman, L.S., Gordon, I.E., Barbe, A., Benner, D.C., Bernath, P.F., Birk, M., Boudon, V., Brown, L.R., Campargue, A., and Champion, J.-P., (2009). The HITRAN 2008 molecular spectroscopic database. J. Quant. Spectrosc. Radiat. Transf.. 110:533-572.

Rothman, L.S., Jacquemart, D., Barbe, a., Chris Benner, D., Birk, M., Brown, L.R., Carleer, M.R., Chackerian, C., Chance, K., Coudert, L.H., et al., (2005). The HITRAN 2004 molecular spectroscopic database. J. Quant. Spectrosc. Radiat. Transf.. 96:139-204.

Rothman, L.S., Rinsland, C.P., Goldman, A., Massie, S.T., Edwards, D.P., Flaud, J.M., Perrin, A., Camy-Peyret, C., Dana, V., Mandin, J.-Y., et al., (1998). The HITRAN molecular spectroscopic database and HAWKS (HITRAN atmospheric workstation): 1996 edition. J. Quant. Spectrosc. Radiat. Transf.. 60:665-710.

Ruiz-Garcia, L., Lunadei, L., Barreiro, P., and Robla, I., (2009). A Review of Wireless Sensor Technologies and Applications in Agriculture and Food Industry: State of the Art and Current Trends. Sensors. 9:4728-4750.

Saarela, J., Toivonen, J., Manninen, A., Sorvajärvi, T., and Hernberg, R., (2009). Wavelength modulation waveforms in laser photoacoustic spectroscopy. Appl. Opt.. 48:743-747.

Sanders, S.T., Baldwin, J. a., Jenkins, T.P., Baer, D.S., and Hanson, R.K., (2000). Diodelaser sensor for monitoring multiple combustion parameters in pulse detonation engines. Proc. Combust. Inst. 28:587-594.

Schiff, H.I., Hastie, D.R., Mackay, G.I., Iguchi, T., and Ridley, B.A., (1983). Tunable diode laser systems for measuring trace gases in tropospheric air. Environ. Sci. Technol.. 17:352A-364A.

Schilt, S., Thévenaz, L., and Robert, P., (2003). Wavelength modulation spectroscopy: combined frequency and intensity laser modulation. Appl. Opt. 42:6728-6738.

Schoonbaert, S.B. and Johnson, M.R., (2011). Fibre-Based TDLS Sensor Network for Detecting Fugitive Emission Sources. In AWMA Annual Conference \& Exhibition. Orlando, Air \& Waste Management Association, p.6.

Schoonbaert, S.B., Tyner, D.R., and Johnson, M.R., (2014a). Remote ambient methane monitoring using fiber-optically coupled optical sensors. Appl. Phys. B.

Schoonbaert, S.B., Tyner, D.R., and Johnson, M.R., (2014b). Ambient Gas Quantification Using Multipoint Optical Detection. In AWMA Annual Conference \& Exhibition. Long Beach, CA, Air \& Waste Management Association.

Schubert, E.F., (2003). Light-Emitting Diodes 1st ed. New York, Cambridge university Press.

Shemshad, J., (2015). Analysis of inaccuracy induced by intensity variation of a DFB laser in fibre optic multipoint 2f-WMS measurements of methane near $1666 \mathrm{~nm}$. Sensors Actuators A Phys. . 222:96-101. 
Shemshad, J., Aminossadati, S.M., and Kizil, M.S., (2012). A review of developments in near infrared methane detection based on tunable diode laser. Sensors Actuators $B$ Chem.. 171-172:77-92.

Sigrist, M.W., Bartlome, R., Marinov, D., Rey, J.M., Vogler, D.E., and Wächter, H., (2007). Trace gas monitoring with infrared laser-based detection schemes. Appl. Phys. B. 90:289-300.

Silveira, J.P. and Grasdepot, F., (1995). CH4 optical sensor using a $1.31 \mu \mathrm{m}$ DFB laser diode. Sensors Actuators B Chem.. 25:603-606.

Silver, J., (1992). Frequency-modulation spectroscopy for trace species detection: theory and comparison among experimental methods, Appl. Opt. Soc.. 31:707-171.

Silver, J., (2005). Simple dense pattern optical multipass cells. Appl. Opt.. 44:6545-6556.

Silver, J. and Stanton, A., (1988). Optical interference fringe reduction in laser absorption experiments. Appl. Opt. . 27:1914-1916.

Simpson, I.J., Thurtell, G.W., Kidd, G.E., Lin, M., Kanemasu, E.T., Nie, D., Bronson, K.F., and Neue, H.U., (1995). Tunable diode laser measurements of methane fluxes from an irrigated rice paddy field in the Philippines. J. Geophys. Res. . 100:7283-7290.

Smith, C.J., Wang, W., and Wysocki, G., (2013). Real-time calibration of laser absorption spectrometer using spectral correlation performed with an in-line gas cell. Opt. Express. 21:22488.

So, S.G., (2008). Laser Spectroscopic Trace Chemical Sensors for Environmental Sensor Networks and Portable Medical Devices.pdf.

So, S.G., Member, S., Wysocki, G., Frantz, J.P., Tittel, F.K., and Fellow, L., (2006). Development of Digital Signal Processor Controlled Quantum Cascade Laser Based Trace Gas Sensor Technology. October. 6:1057-1067.

Song, K. and Jung, E.C., (2003). Recent Developments in Modulation Spectroscopy for Trace Gas Detection Using Tunable Diode Lasers. Appl. Spectrosc. Rev. . 38:395432.

Spectralcalc, (1994). Calculation of molecular spectra with the Spectral Calculator. http://www.spectralcalc.com/info/CalculatingSpectra.pdf

Stewart, G., Johnstone, W., Thursby, G., and Culshaw, B., (2010). Near Infrared Spectroscopy for Fibre Based Gas Detection. In A. A. Kazemi, B. C. Kress, \& E. Y. Chan, eds. Proc. SPIE 7675, Photonics in the Transportation Industry: Auto to Aerospace III. Orlando, FL, pp.1-9.

Stewart, G., Shields, P., and Culshaw, B., (2004). Development of fibre laser systems for ring-down and intracavity gas spectroscopy in the near-IR. Science (80-. ).. 15:1621-1628.

Stewart, G., Tandy, C., Moodie, D., Morante, M.A., and Dong, F., (1998a). Design of a fibre optic multi-point sensor for gas detection. Sensors Actuators B Chem.. 51:227-232. 
Stewart, G., Tandy, C., Moodie, D., Morante, M.A., and Dong, F., (1998b). Design of a fibre optic multi-point sensor for gas detection. Sensors Actuators B Chem. . $51: 227-232$.

Stutz, J. and Platt, U., (1996). Numerical analysis and estimation of the statistical error of differential optical absorption spectroscopy measurements with least-squares methods. Appl. Opt.. 35:6041-6053.

Sun, H.C. and Whittaker, E.A., (1992). Novel etalon fringe rejection technique for laser absorption spectroscopy. Appl. Opt.. 31:0-4.

Tai, H., Yamamoto, K., Uchida, M., Osawa, S., and Uehara, K., (1992). Long-distance simultaneous detection of methane and acetylene by using diode lasers coupled with optical fibers. IEEE Photonics Technol. Lett.. 4:804-807.

Triki, M., Nguyen Ba, T., and Vicet, A., (2015). Compact sensor for methane detection in the mid infrared region based on Quartz Enhanced Photoacoustic Spectroscopy. Infrared Phys. Technol. 69:74-80.

Tyner, D.R., (2015). Private Communication.

Uehara, K. and Tai, H., (1992). Remote detection of methane using a 1.66 um diode laser. Appl. Opt. 31:pp809-814.

Uehara, K., Tai, H., and Kimura, K., (1997). Real-time monitoring of environmental methane and other gases with semiconductor lasers: a review. Sensors Actuators B Chem. 39:136-140.

UNFCCC, (2014). GHG data from UNFCCC. http://unfccc.int/ghg_data/ghg_data_unfccc/items/4146.php

US EPA, (2006). VOC Fugitive Losses: New Monitors, Emission Losses, and Potential Policy Gaps. 2006 International Workshop, Washington, D.C., United States Environmental Protection Agency (US EPA). Available at: http://www.epa.gov/ttnchie1/efpac/documents/wrkshop_fugvocemissions.pdf

US EPA, (2008). Alternative Work Practice to Detect Leaks from Equipment. 40 CFR Parts 60, 63, and 65; RIN 2060-AL98, Washington, D.C., United States Environmental Protection Agency (US EPA). Available at: http://www.gpo.gov/fdsys/pkg/FR-2008-12-22/pdf/E8-30196.pdf

US EPA, (2015). Method 21 - Determination of Volatile Organic Compound Leaks.

Ventrudo, B.F. and Cassidy, D.T., (1990). Operating characteristics of a tunable diode laser absorption spectrometer using short-external-cavity and DFB laser diodes. Appl. Opt.. 29:5007-5013.

Webster, C.R., (1988). Stratospheric Composition Measurements of Earth and Titan using High-Resolution Tunable Diode Laser Spectroscopy. Jet Propuls.. 40:239248.

Wei, Y., Chang, J., Lian, J., and Liu, T., (2015). A Coal Mine Multi-Point Fiber Ethylene Gas Concentration Sensor. Photonic Sensors. 5:67-71. 
Weiguang, M., In, W.Y., Ong, L.D., Ang, L.W., I, C.L., and Ia, S.J., (2005). HighSensitivity Detection of Methane Near $6106 \mathrm{~cm}-1$ Using Tunable External-Cavity Diode Laser. Jpn. J. Appl. Phys.. 44:1961-1965.

Werle, P., (1998). A review of recent advances in semiconductor laser based gas monitors. Spectrochem. Acta Part A. 54:197 - 236.

Werle, P., Mazzinghi, P., D’Amato, F., De Rosa, M., Maurer, K., and Slemr, F., (2004). Signal processing and calibration procedures for in situ diode-laser absorption spectroscopy. Spectrochim. Acta. A. Mol. Biomol. Spectrosc.. 60:1685-705.

Werle, P., Mucke, R., and Slemr, F., (1993). The limits of signal averaging in atmospheric trace-gas monitoring by tunable diode-laser absorption spectroscopy (TDLAS). Appl. Phys. B. 57:131-139.

Werle, P., Scheumann, B., and Schandal, J., (1994). Real-time signal-processing concepts for trace-gas analysis by diode-laser spectroscopy. Engineering. 33:3093.

Werle, P., Slemr, F., Gehrtz, M., and Bräuchle, C., (1989). Wideband noise characteristics of a lead-salt diode laser: possibility of quantum noise limited TDLAS performance. Appl. Opt.. 28:1638-42.

White, J.U., (1942). Long Optical Paths of Large Aperture. J. Opt. Soc. Am.. 32.

Whitenett, G., Stewart, G., Atherton, K., Culshaw, B., and Johnstone, W., (2003). Optical fibre instrumentation for environmental monitoring applications. J. Opt. A Pure Appl. Opt.. 5:S140-S145.

Wienhold, F., Frahm, H., and Harris, G., (1994). Measurements of N2O fluxes from fertilized grassland using a fast response tunable diode laser spectrometer. $J$. Geophys. Res.. 99:16557-67.

Wysocki, G., Bakhirkin, Y., So, S., Tittel, F.K., Hill, C.J., Yang, R.Q., and Fraser, M.P., (2007). Dual interband cascade laser based trace-gas sensor for environmental monitoring. Appl. Opt.. 46:8202-8210.

Ye, J., Ma, L.-S., and Hall, J.L., (1998). Ultrasensitive detections in atomic and molecular physics: demonstration in molecular overtone spectroscopy. J. Opt. Soc. Am. B. 15:6.

Ye, W.-L., Zheng, C.-T., Cheng, X.-Y., Wu, F.-P., Chen, M.-M., and Wang, Y.-D., (2015). Mid-infrared ppm-level methane detection device using small-size absorption pool and dual-channel lock-in amplifier. Infrared Phys. Technol.. 71:339-346.

Yu, H.B., Jin, W., Ho, H.L., Chan, K.C., Chan, C.C., Demokan, M.S., Stewart, G., Culshaw, B., and Liao, Y.B., (2001). Multiplexing of optical fiber gas sensors with a frequency-modulated continuous-wave technique. Appl. Opt.. 40:10111020 .

Zhang, T., Wang, W., Gao, L., Koscica, T., and Li, D., (2012). Development of eightchannel methane gas optical fiber sensing system. In Proc. SPIE 8417, 6th International Symposium on Advanced Optical Manufacturing and Testing 
Technologies: Optical Test and Measurement Technology and Equipment. pp.16.

Zhang, W., Liu, R., Zhang, W., Jia, H., and Xu, K., (2013). Discussion on the validity of NIR spectral data in non-invasive blood glucose sensing. Biomed. Opt. Express. 4:789-802. 


\section{Appendix A: Test Chamber}

\section{A.1 Gas Mixing System}

The gas mixing system was designed to generate accurate volume mixing ratios of methane at low flow rates to simulate fugitive methane plumes. Depending on the test being conducted, varying concentrations of methane were generated by combining air or nitrogen with pre-mixed calibration gas using pre-calibrated mass-flow controllers (MFCs). Figure A.1shows a schematic of the gas mixing system.

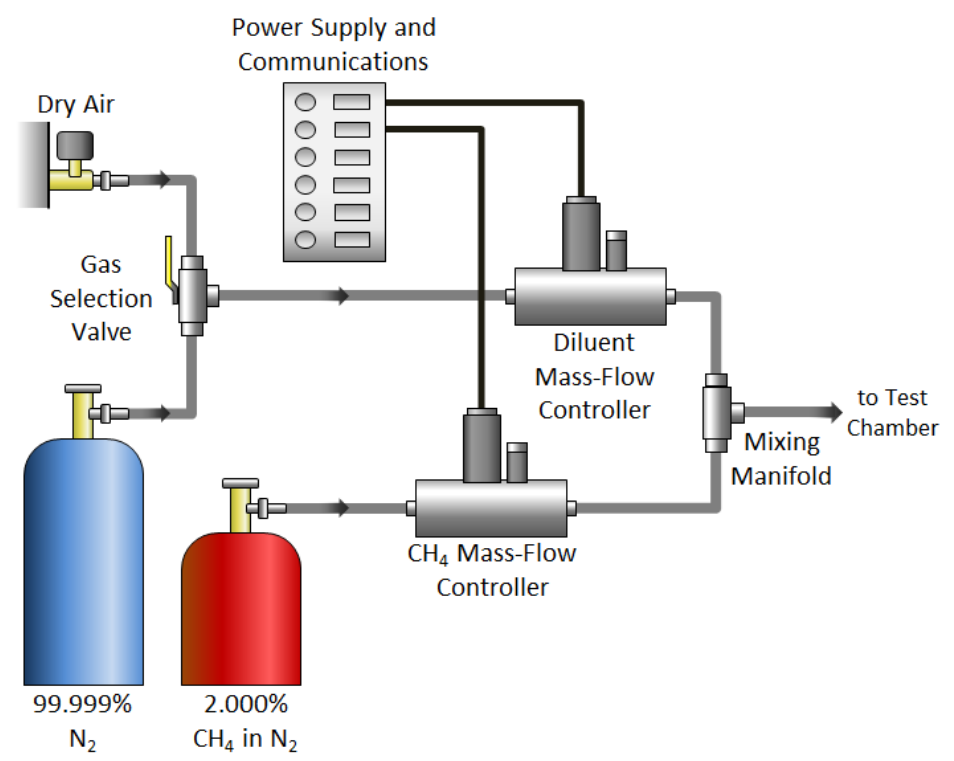

Figure A.1: Gas mixing system schematic

Unless otherwise stated, calibration-grade $2.0 \%$ methane in nitrogen was combined with air from the building compressed air lines for all tests. High-purity, dry, hydrocarbonfree nitrogen was used in place of compressed air for specific absorption-free system calibrations, and to establish critical system performance baselines. Manufacturer's specifications of the $2.0 \%$ methane mix, high-purity nitrogen, and measured data for the building compressed air lines (See Section A.2.1) are presented in Table A.1. 
Table A.1: Bottled gas and compressed air specifications

\begin{tabular}{|c|c|c|c|c|}
\hline Parameter & Units & $\begin{array}{c}100 \% \\
\text { Nitrogen }\end{array}$ & $\mathrm{CH}_{4}$ in $\mathrm{N}_{2}$ & Compressed Air \\
\hline $\mathrm{CH}_{4}$ Concentration & $\mathrm{ppm}_{\mathrm{v}}$ & - & 20,000 & $2.59 \dagger$ \\
\hline Accuracy & - & $\pm 0.001 \%$ & $\pm 0.02 \%$ & $\pm 0.56 \mathrm{ppm}_{\mathrm{v}}^{\dagger}$ \\
\hline
\end{tabular}

Depending on the flow rates and methane concentrations required, five different MFCs were used in the test system as specified in Table A.2. By using different combinations of MFCs and compressed gases, the gas mixing system was able to deliver methane volume mixing ratios between 0.25 and $750 \mathrm{ppm}_{\mathrm{v}}$ at flow rates between 100 and 1000 SLPM, as illustrated in Figure A.2.

Table A.2: Mass-flow controller specifications

\begin{tabular}{|c|c|c|c|c|c|}
\hline Parameter & $\begin{array}{c}1000 \mathrm{SLPM} \\
\mathrm{N}_{2} \\
\end{array}$ & $\begin{array}{c}1000 \text { SLPM } \\
\text { Air } \\
\end{array}$ & $\begin{array}{c}0.5 \mathrm{SLPM}^{2} \% \mathrm{CH}_{4} \\
\end{array}$ & $\begin{array}{c}2.0 \mathrm{SLPM}^{2} \\
2 \% \mathrm{CH}_{4} \\
\end{array}$ & $\begin{array}{c}10.0 \mathrm{SLPM} \\
2 \% \mathrm{CH}_{4} \\
\end{array}$ \\
\hline Model Number & \multirow{2}{*}{\multicolumn{2}{|c|}{$\begin{array}{c}\text { SLA5853S2EAB2A2A1 } \\
0111080470021001\end{array}$}} & SLA5850S & $5850 \mathrm{~S}$ & SLS5850S \\
\hline Serial Number & & & 01B20350472 & 0107060341313 & 0111080470030001 \\
\hline Calibration Gas & Nitrogen & Dry Air & \multicolumn{3}{|c|}{$2.0 \%$ Methane in Nitrogen } \\
\hline Calibration Type & Factory & $\begin{array}{l}\text { Coriolis Flow- } \\
\text { Meter }\end{array}$ & \multicolumn{3}{|c|}{ Bubble Flow-Meter } \\
\hline $\begin{array}{l}\text { Effective Range } \\
\text { (SLPM) }\end{array}$ & $100-1000$ & $100-1000$ & $0.025-0.5$ & $0.1-2.0$ & $0.5-10.0$ \\
\hline Accuracy $(95 \%)$ & $\pm 0.75 \%$ & $\pm 0.80 \%$ & $\pm 1.26 \%$ & $\pm 1.15 \%$ & $\pm 1.25 \%$ \\
\hline
\end{tabular}

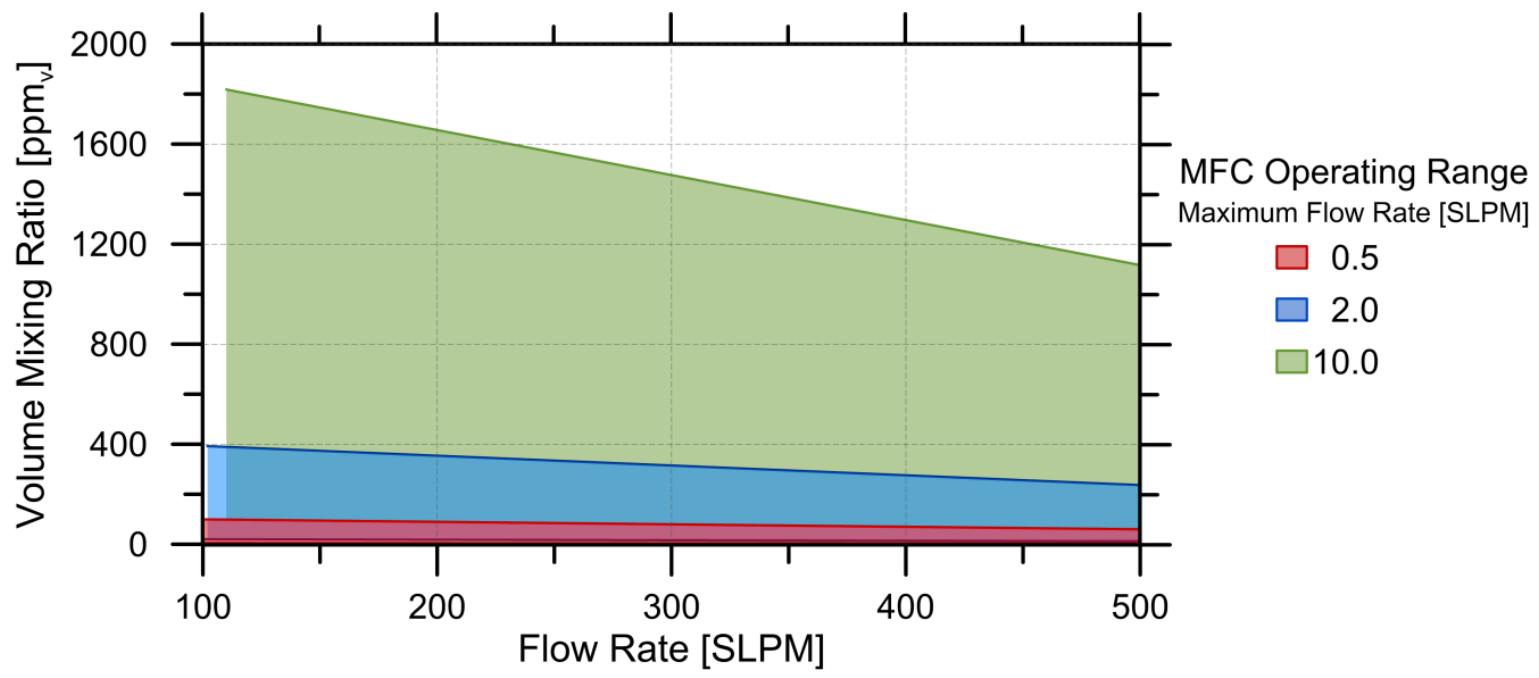

Figure A.2: Gas Mixing System Methane Concentration and Flow Rate Operating Range 
The requested methane volume mixing ratio uncertainty was calculated by combining in quadrature the uncertainties from pre-mixed calibration gas, the diluent, and the MFCs. To minimize the methane concentration uncertainty, the lower range of the MFCs was avoided whenever possible such that the 0.5 SLPM methane MFC was used for volume mixing ratios between 2 and $50 \mathrm{ppm}_{\mathrm{v}}$, and the 2.0 SLPM controller was used between 20 and $200 \mathrm{ppm}_{\mathrm{v}}$. For most experiments presented in this thesis, the gas mixing system was set at 400 SLPM.

\section{A.2 Test System Performance}

\section{A.2.1 Volume Mixing Ratio Uncertainty}

To verify the performance of the gas mixing system, generated methane concentrations were separately checked using a Fourier transform infrared spectroscopy (FTIR) gas analyzer (MKS Multi-Scan 2030). The FTIR methane measurement specifications are listed in Table A.3.

Table A.3: Fourier Transform Infrared Spectroscopy System Specifications

\begin{tabular}{l|c|c}
\hline \multicolumn{1}{c|}{ Parameter } & Units & Value \\
\hline Minimum Detection Limit & $\mathrm{ppm}_{\mathrm{v}}$ & 0.6 \\
Uncertainty (95\%) & $\mathrm{ppm}_{\mathrm{v}}$ & 1.2 \\
Sample Rate & $\mathrm{Hz}$ & 1 \\
Spectral Resolution & $\mathrm{cm}^{-1}$ & 0.5 \\
\hline
\end{tabular}

The FTIR system was first used to quantify the variation of the methane concentration within the building compressed air lines. As reported in Figure A.3, the methane concentration in the compressed air source was analyzed for over 6 hours on three separate days. The time-weighted mean volume fraction of methane in the compressed air was $2.59 \pm 0.56 \mathrm{ppm}_{\mathrm{v}}$ with $95 \%$ confidence. As might be expected in an urban setting, this is slightly higher than the mean ambient methane concentrations of $1.83 \mathrm{ppm}_{\mathrm{v}}$ (as stated by the Department of Earth Sciences at the University of London (Dlugokencky et al., 2011) and NOAA Earth System Research Laboratory in Boulder Colorado (Kirschke et al., 2013)). 


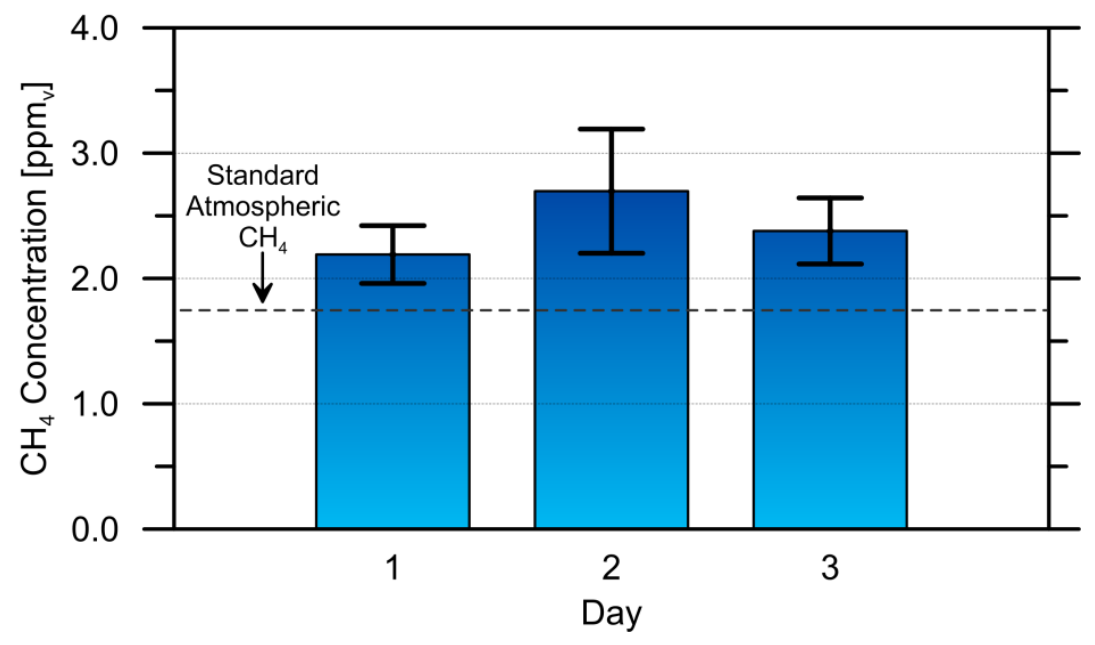

Figure A.3: Compressed air methane concentration observed over three days

The FTIR was also used to check the accuracies of methane volume mixing ratios generated by the gas mixing system. Time-synchronized FTIR and MFC measurements were taken at volume mixing ratios between 0.5 and $100 \mathrm{ppm}_{\mathrm{v}}$, after a settling time of at least 10 minutes for each measurement. Figure A.4 compares the FTIR methane volume mixing ratio (VMR) measurements and the requested methane VMR from the gas mixing system MFCs (averaged for 1 second) at a constant flow rate of 400 SLPM. Figure A.4a shows results using the 0.5 SLPM MFC, whereas Figure A.4b shows results using the 2.0 SLPM MFC. 
a) 0.5 SLPM Maximum Flow Rate MFC

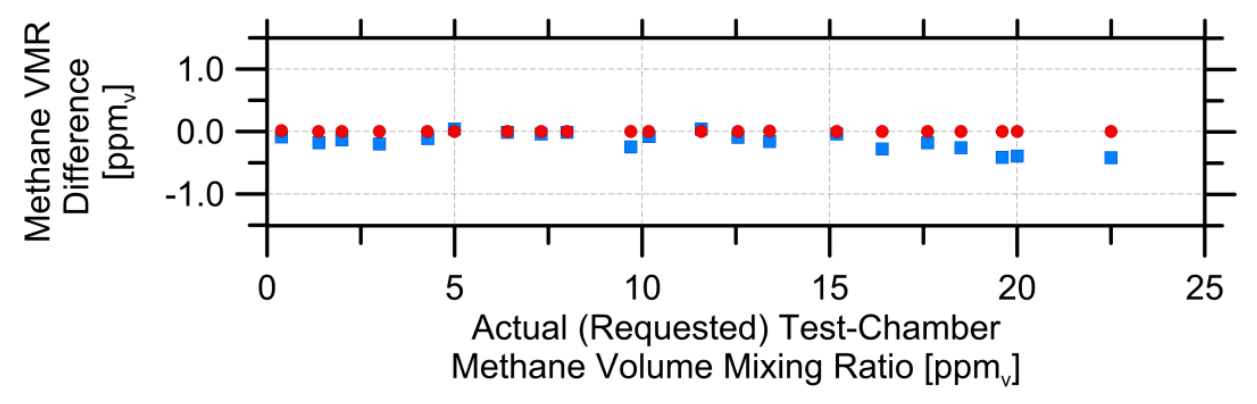

b) 2.0 SLPM Maximum Flow Rate MFC

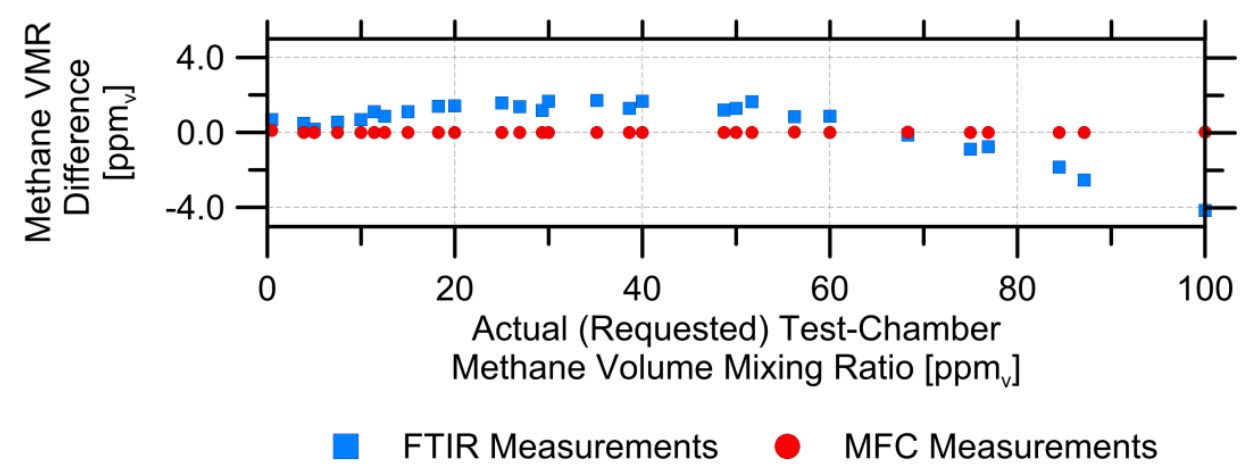

Figure A.4: Residual between FTIR methane volume mixing ratio (VMR) measurements and requested methane VMR from the gas mixing system MFCs (averaged for 1 second) at a constant flow rate of 400 SLPM at ambient temperature and pressure. a) 0.5 SLPM MFC and b) 2.0 SLPM MFC

For experiments using the 0.5 SLPM MFC, the maximum bias between the FTIR and the MFC measurements was $-0.42 \mathrm{ppm}_{\mathrm{v}}$ at $22.5 \mathrm{ppm}_{\mathrm{v}}\left(\right.$ mean bias of $0.16 \mathrm{ppm}_{\mathrm{v}}$ ). For experiments using the 2.0 SLPM MFC, the maximum bias was $-4.18 \mathrm{ppm}_{\mathrm{v}}$ at $100 \mathrm{ppm}_{\mathrm{v}}$ (mean bias of $<0.51 \mathrm{ppm}_{\mathrm{v}}$ ). Based on these results, the calculated gas mixtures provided by the MFCs were deemed sufficiently accurate to be used to test the performance of the TDLAS system. Figure A.5 illustrates the estimated methane volume mixing ratio uncertainties calculated from the MFC specifications, for requested methane volume mixing ratios between 1 and $100 \mathrm{ppm}_{\mathrm{v}}$ at 400 SLPM. Absolute uncertainties (plotted in $\mathrm{ppm}_{\mathrm{V}}$ ) are shown on the left axis whereas relative uncertainties (plotted as a percent of the requested volume mixing ratio) are plotted on the right axis. 


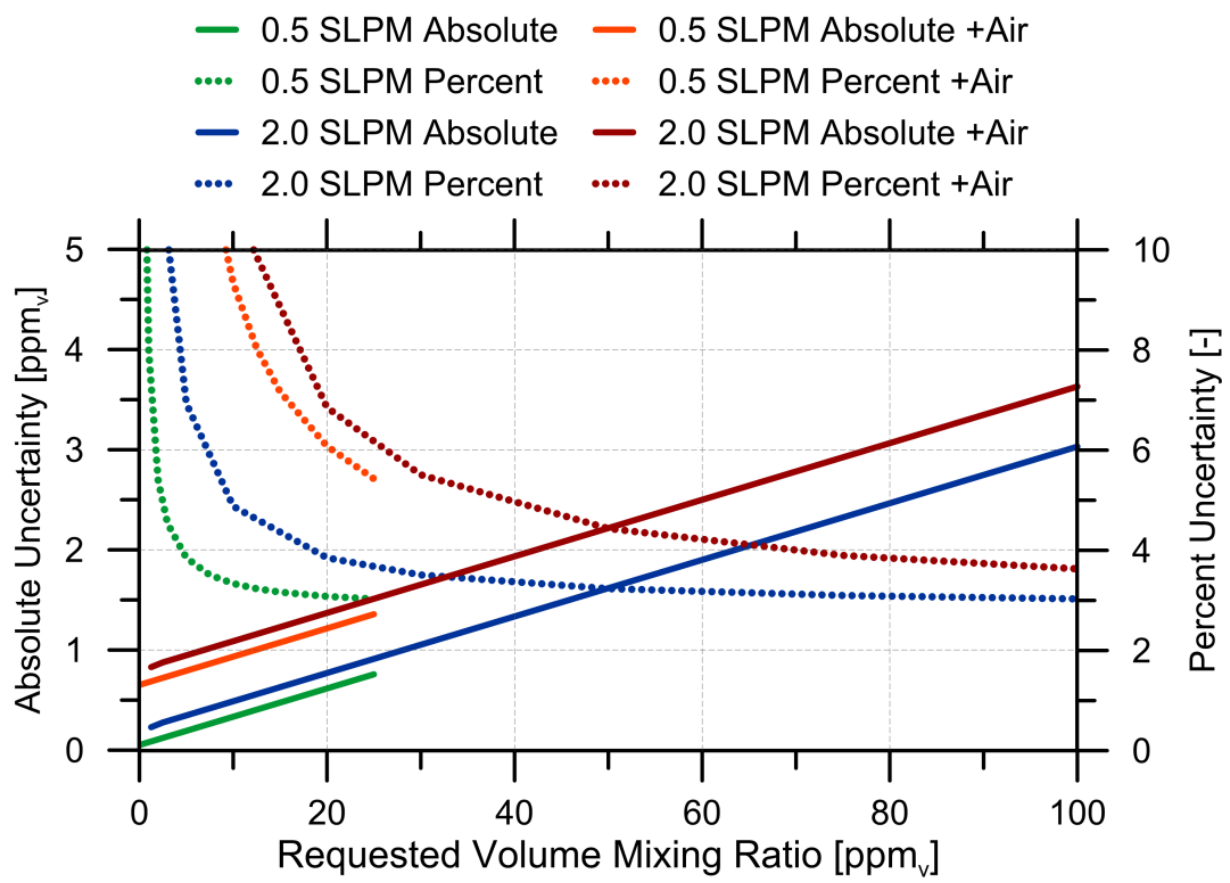

Figure A.5: Mixed gas methane volume mixing ratio uncertainty

When the $2.0 \%$ methane was diluted with nitrogen, the uncertainty in the generated methane volume mixing ratio was governed by the MFC uncertainty, linearly increasing from approximately $0.25 \mathrm{ppm}_{\mathrm{v}}$ to $3.05 \mathrm{ppm}_{\mathrm{v}}$ at requested methane volume mixing ratios of 2 and $100 \mathrm{ppm}_{\mathrm{v}}$ respectively. Alternatively, when the $2.0 \%$ methane was diluted with compressed air, the resultant methane volume mixing ratio uncertainty increased to 0.75 $\mathrm{ppm}_{\mathrm{V}}$ and $3.55 \mathrm{ppm}_{\mathrm{V}}$ at requested concentrations of 2 and $100 \mathrm{ppm}_{\mathrm{V}}$ respectively.

\section{A.2.2 Settling Time}

Once a specific methane volume mixing ratio was requested, there was a time delay before that concentration was observable within the test section. This time delay was dominated by the MFC settling time and the time for bulk gas transport through the piping system and mixing sections of the test chamber. To quantify this settling time, the change in the WMS second-harmonic value was measured for a step-change in requested volume mixing ratio. Figure A.6 plots 1 -s and 15-s averaged measured $2 f$ values for a sample series of requested step changes methane concentration from 2 to $50 \mathrm{ppm}_{\mathrm{v}}$ and 2 
to $100 \mathrm{ppm}_{\mathrm{v}}$. Figure A.7 illustrates the time required for the 10 -second average $2 f$-peak value to settle within $2.5 \%$ of a steady-state value, after a methane concentration change was requested. A constant mass-flow rate of 200 SLPM was selected to conserve calibration gas, and to provide a worst-case settling-time compared to the standard flowrate of 400 SLPM used in most experiments.

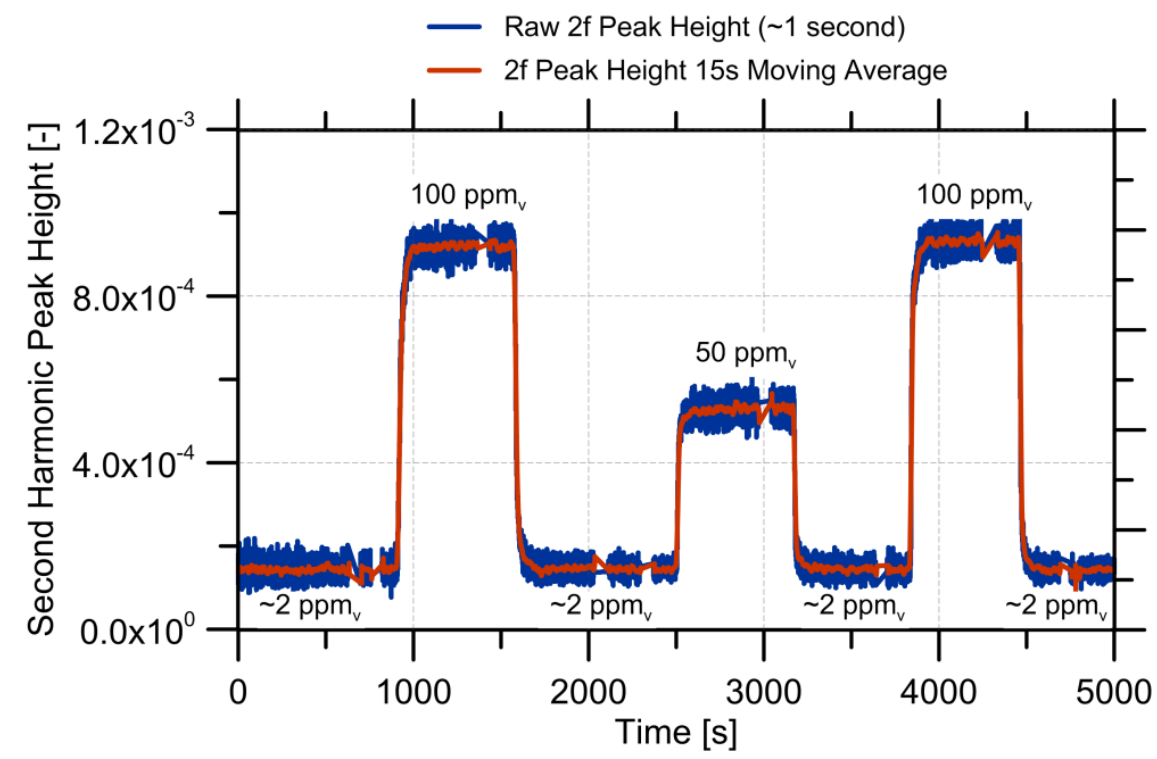

Figure A.6: $2 f$ Peak height response to step changes in requested concentration

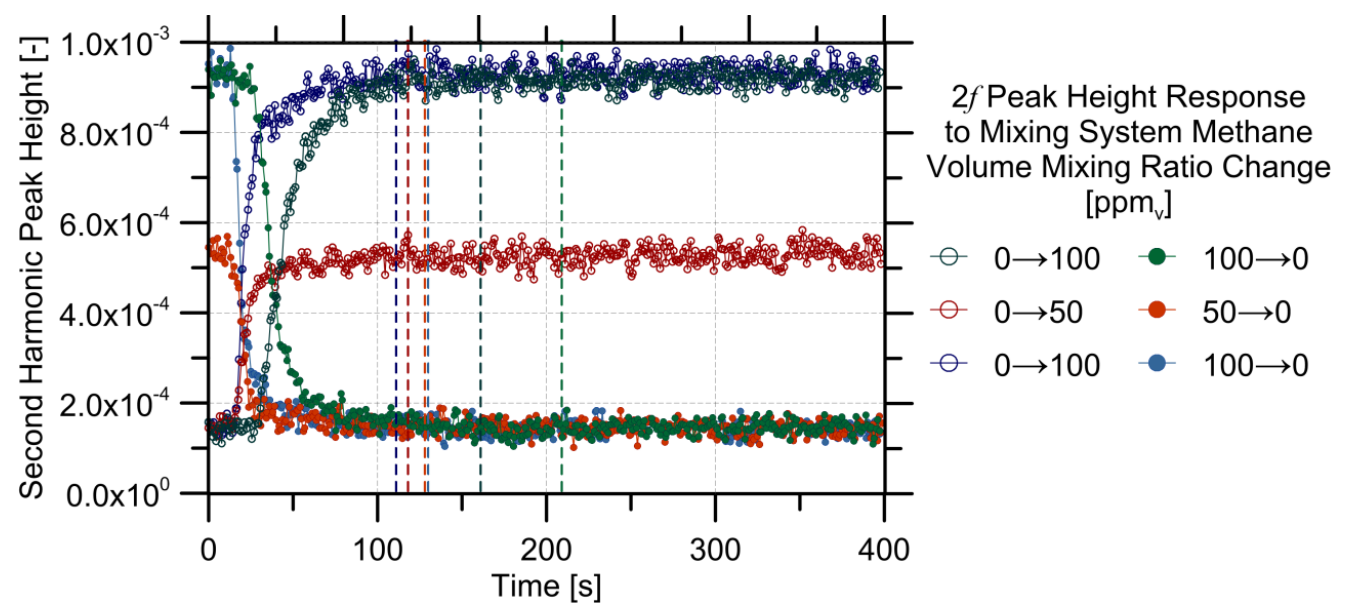

Figure A.7: Measured $2 f$ value settling time for requested concentration step-changes of 100 and $50 \mathrm{ppm}_{\mathrm{v}}$ at $200 \mathrm{SLPM}$ 
At a flow rate of 200 SLPM, the maximum settling time was approximately 3.5 minutes, with an average settling time for the 6 runs of 2.5 minutes. The settling time for decreasing concentrations was slightly longer than for increasing concentrations. Thus, to ensure stable concentrations during experiments, a minimum settling time of 6 minutes was used for all experimental tests with volume flow rates at 200 SLPM, and 3 minutes for volume flow rates greater than or equal to 400 SLPM. Finally, to reduce the influence of air flow disturbances within the laboratory propagating into the test-chamber, a plastic bag was loosely attached to the end of the test chamber. 


\section{Appendix B: Calibration Methods}

As described in Section 4.6, the measured methane volume mixing ratio (VMR), $q$, was derived from laser absorption (SI) or $2 f$ signals (WMS) using either a theory-based estimation of the measureable volume mixing ratios (termed a "theory-based calibration") or an experimental calibration. This appendix summarizes these calibration methods. In each case presented below the measured absorption or $2 f$ values $\left(x_{a b s}\right.$ and $\left.x_{2 f}\right)$ were computed at the center wavenumber of the absorption feature, $v_{p}$, for further details see Section 2.2.

\section{B.1 Theory-Based Calibration Method}

The theory-based calibration relied on spectroscopic theory to calculate methane volume mixing ratios from measured absorption or $2 f$ values, estimated absorption-free intensity signals, and ambient temperature and pressure values. This predicted theory-based SI and WMS methane VMR was then related to actual methane VMR via an empirical calibration, which accounted for uncertainties in the spectroscopic theory and available absorption line data.

\section{B.1.1 Look-up Tables Used in the Theory-Based Calibration}

To permit real-time volume mixing ratio measurement with low computational effort, the theory-based calibration approach was implemented using pre-computed look-up tables, which were used to infer methane VMR from measured absorption or $2 f$ signals at a fixed optical path length $(5.70 \mathrm{~m})$, and a given pressure and temperature. The SI look-up table included theoretical approximations of SI absorption values, which were calculated using Equations 2.15 and 2.16. The WMS look-up table included the first five Fourier coefficients, $\left\{H_{0}, \ldots, H_{4}\right\}$ (Equation 2.24), from which the $2 f$ values were computed. Both the SI and WMS tables spanned the range of conditions listed in Table B.1. The tables enabled estimation of a methane VMR as a function of absorption or $2 f$ value, pressure, and temperature at a fixed optical path-length of $5.7 \mathrm{~m}$. 
Table B.1: Look-up table parameters and resolution

\begin{tabular}{l|c|r|r|r}
\hline \multicolumn{1}{c|}{$\begin{array}{c}\text { Look-up Table } \\
\text { Parameter }\end{array}$} & \multirow{2}{*}{ Units } & \multicolumn{3}{|c}{ Range } \\
\hline Pressure & $\mathrm{kPa}$ & \multicolumn{1}{c}{ Low } & \multicolumn{1}{c}{ High } & Resolution \\
Temperature & $\mathrm{K}$ & 293.0 & 103.0 & 0.25 \\
& & 1.0 & 300.0 & 0.5 \\
& & 30.0 & 25.0 & 1.0 \\
Methane Volume & $\mathrm{ppm}_{\mathrm{v}}$ & 275.0 & 550.0 & 5.0 \\
Mixing Ratio & & 600.0 & 1400.0 & 25.0 \\
& & 1600.0 & 4400.0 & 50.0 \\
& & 5.7 & $\mathrm{n} / \mathrm{a}$ & $\mathrm{n} / \mathrm{a}$ \\
\hline
\end{tabular}

Because of computational and file-size constraints, the resolution of the look-up data was necessarily limited as indicated in Table B.1. The potential error when interpolating values from the SI and WMS look-up tables was estimated by calculating the theoretical ${ }^{4}$ absorption or $2 f$ values for 40,000 random combinations of pressure, temperature, and methane volume mixing ratio equally distributed over the full ranges of each parameter. The maximum absorption value error at methane VMR values between 1 and $200 \mathrm{ppm}_{\mathrm{v}}$ was $0.003 \%$, occurring at a VMR of $\sim 30 \mathrm{ppm}_{\mathrm{v}}$ as illustrated in Figure B.1.

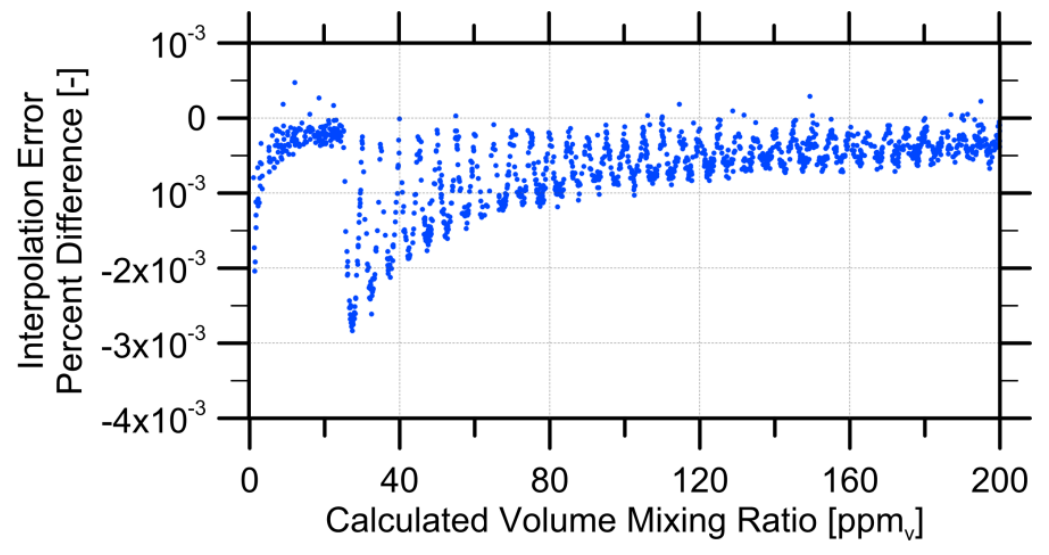

Figure B.1: SI look-up table interpolation error between 1 and 200 ppm $_{\mathrm{v}}$

\footnotetext{
${ }^{4}$ Using the same Lorentzian-based approximations of the absorption and $2 f$ values at the maximum of the $2 v_{3} \mathrm{R}(3)$ methane absorption feature used to originally construct the table
} 
Similarly, as shown in Figure B.2 the maximum interpolation error when extracting $2 f$ values from the look-up table was $0.004 \%$, occurring at a methane VMR of $\sim 107_{\mathrm{v}}^{\mathrm{ppm}}$.

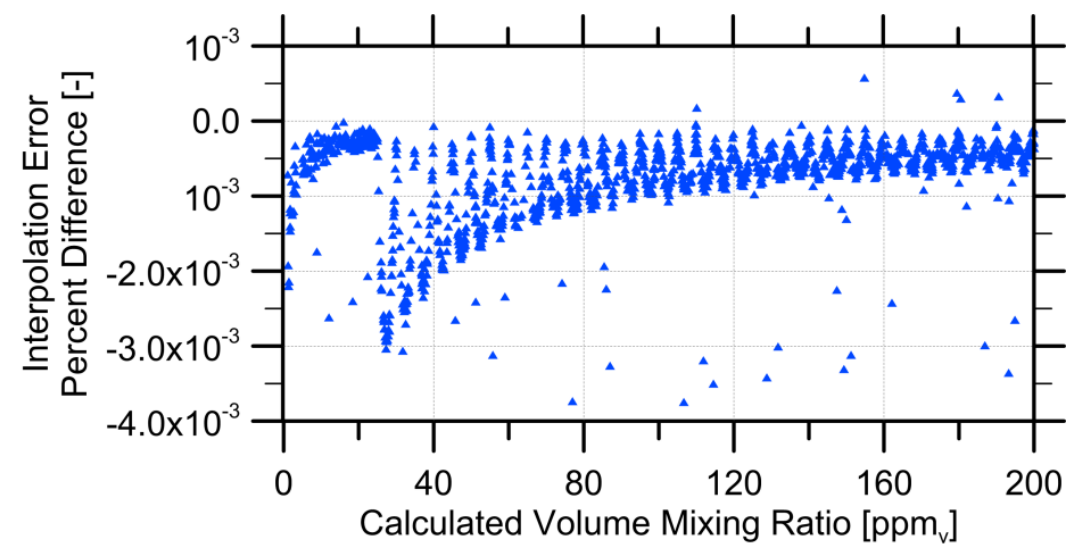

Figure B.2: WMS look-up table interpolation error between 1 and 200 ppm $_{\mathrm{v}}$

Figure B.3 plots predicted SI and WMS methane VMR datasets calculated using the look-up tables as a function of actual (requested) volume mixing ratio in the test chamber. These example datasets was obtained over an 8 hour period, with test chamber pressures between $98 \mathrm{kPa}$ and $103 \mathrm{kPa}$, temperatures between $21^{\circ} \mathrm{C}$ and $25^{\circ} \mathrm{C}$, and with the $5.79 \mathrm{~m}$ optical sensor head coupled to the base station with a 6-m $9 / 125 \mu \mathrm{m}$ fiber optic cable. 


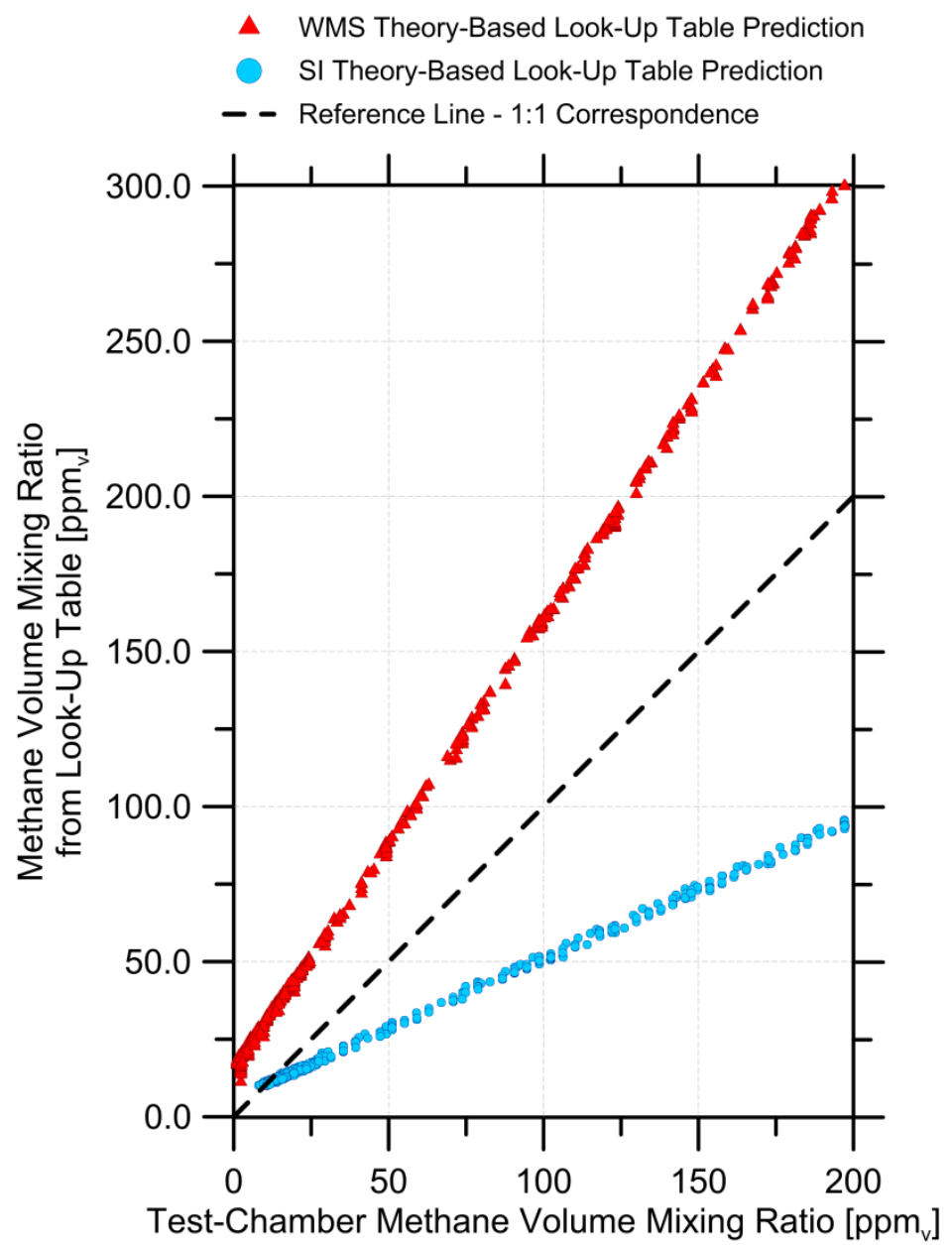

Figure B.3: Comparison of the methane volume mixing ratio predicted using the WMS and SI look-up tables (red and blue points respectively) with actual methane volume mixing ratios in the test chamber. The reference dashed black line is indicates perfect correspondence. Plotted data were obtained with test chamber pressures between $98 \mathrm{kPa}$ and $103 \mathrm{kPa}$, temperatures between $21^{\circ} \mathrm{C}$ and $25^{\circ} \mathrm{C}$, and with the $5.79 \mathrm{~m}$ optical sensor head coupled to the base station with a 6-m 9/125 $\mu \mathrm{m}$ fiber optic cable.

Figure B.3 shows that methane VMR data (for both SI and WMS methods) obtained using the theory-based look-up table are linearly related to the actual testchamber methane VMR, but the correspondence is not perfect. Thus, an empirical correction was subsequently applied (as outlined in Section B.1.2) in the form of a linear least squares fit of the look-up table results to the test-chamber methane VMR data. The need for these empirical corrections is attributable to errors in the spectroscopic data and limits of the theory used to generate the look-up tables 


\section{B.1.2 Empirical Correction to the Theory-Based Look-up Table Output}

As noted in the previous section, to determine the final calibration functions, an empirical correction was applied to the methane volume mixing ratios predicted with the SI or WMS look-up tables. The sample calibration data shown in this section are $1 \mathrm{~s}$ measurements of test chamber volume mixing ratios between $2.0 \mathrm{ppm}_{\mathrm{v}}$ and $200 \mathrm{ppm}_{\mathrm{V}}$ methane in air, collected at the $2 v_{3} \mathrm{R}(3)$ methane absorption manifold. The ambient pressure and temperature in the test chamber was measured between $98 \mathrm{kPa}$ and $103 \mathrm{kPa}$ and $21^{\circ} \mathrm{C}$ and $25^{\circ} \mathrm{C}$, and the thermal enclosure temperature was fixed at $20.1^{\circ} \mathrm{C}$. Data were acquired using the $5.79 \mathrm{~m}$ optical sensor head connected to the base station with a 6-m long, 9/125 $\mu \mathrm{m}$ fiber.

\section{SI Technique}

The absorption value, $x_{\mathrm{abs}}\left(I_{0}, I\right)$, is computed via Equation 2.18, using the estimated absorption-free intensity and known laser intensity and wavelength response characteristics $\left(I_{0}, i_{0}, i_{2}, \psi_{1}, \psi_{2}\right)_{\mathrm{SI}}$ following Equations 2.15 and 2.16. The SI empirical correction function converts the predicted volume mixing ratio from the SI theory-based look-up table, $\operatorname{LUT}_{\mathrm{SI}}\left(x_{a b s}, T_{\mathrm{amb}}, P_{\mathrm{amb}}\right)$, to a final measured methane volume mixing ratio, $q$, using an experimentally determined linear calibration factor, $G$, and zeroresponse, $b$, following:

$$
q=G\left(\operatorname{LUT}_{\mathrm{SI}}\left(x_{\mathrm{abs}}, T_{\mathrm{amb}}, P_{\mathrm{amb}}\right)\right)-b
$$

where $T_{\mathrm{amb}}$ and $P_{\mathrm{amb}}$ are the measured temperature and pressure in the test chamber.

Figure B.5 illustrates the residual between the final SI measured volume mixing ratio (after applying the theory-based lookup table and the empirical correction) and the actual VMR in the test-chamber. The dashed lines show the $95 \%$ confidence intervals and prediction intervals. With the system fully calibrated in this manner, it was then possible to precisely calculate measurement precision and lower detection limit (LDL) as detailed in Appendix C. 


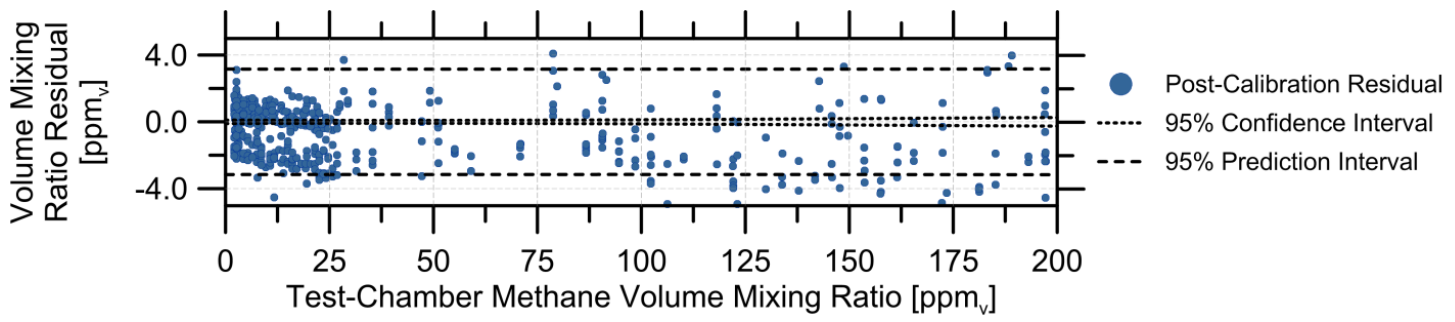

Figure B.4: Calibration residuals for SI method 1-second averaged methane volume mixing ratio values obtained using the theory-based calibration approach (which includes application of the empirical correction). Dashed and dotted lines indicate $95 \%$ prediction and confidence intervals of the final calibration. Plotted data were collected for ambient pressures and temperatures in the test chamber between $98 \mathrm{kPa}$ and $103 \mathrm{kPa}$ and $21^{\circ} \mathrm{C}$ and $25^{\circ} \mathrm{C}$. The thermal enclosure temperature was fixed at $20.1^{\circ} \mathrm{C}$ and data were acquired using the $5.79 \mathrm{~m}$ optical sensor head connected to the base station with a 5-m long, 9/125 $\mu \mathrm{m}$ fiber.

\section{2f-WMS Technique}

The measured $2 f$ value, $x_{2 f}$, is computed from the measured optical sensor head return intensity at the wavenumber of the absorption manifold maximum, $I\left(v_{\breve{c}}\right)$, following Equations 2.29 to 2.31. The WMS empirical correction function converts the predicted volume mixing ratio from the WMS theory-based look-up table, $\operatorname{LUT}_{\mathrm{WmS}}\left(x_{2 f}, T_{\mathrm{amb}}, P_{\mathrm{amb}}\right)$, to a final measured methane volume mixing ratio, $q$, using an experimentally determined linear calibration factor, $G$, and zero-response, $b$ :

$$
q=G\left(\operatorname{LUT}_{\mathrm{WMS}}\left(x_{2 f}, T_{\mathrm{amb}}, P_{\mathrm{amb}}\right)\right)-b
$$

Figure B.5 illustrates the residual between the final WMS-measured, 1-second averaged methane volume mixing ratio (i.e. after applying the theory-based lookup table and the empirical correction) and the actual VMR in the test-chamber. The dashed and dotted lines show the $95 \%$ prediction intervals and confidence intervals. Performance of the TDLAS system when implementing the WMS theory-based calibration is discussed further in Chapter 5. 


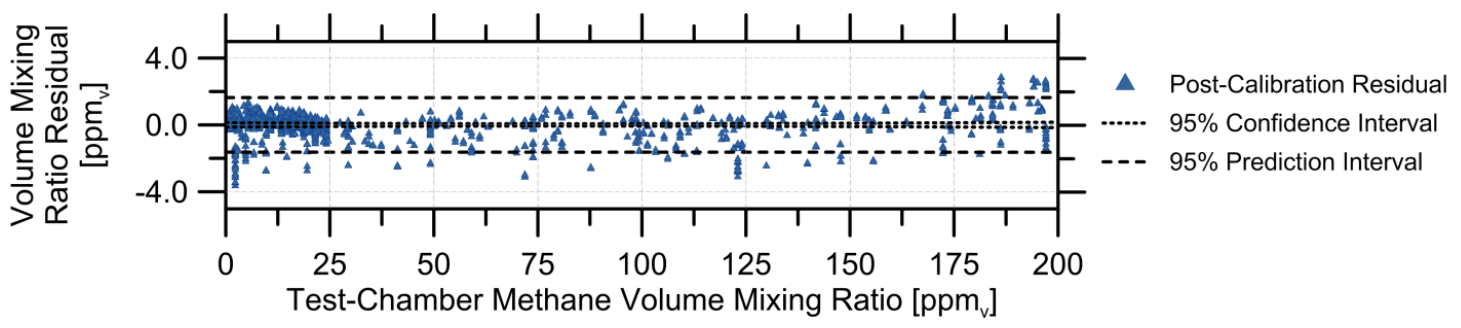

Figure B.5: Calibration residuals for WMS method 1-second averaged methane volume mixing ratio values obtained using the theory-based calibration approach (which includes application of the empirical correction). Dashed and dotted lines indicate $95 \%$ prediction and confidence intervals of the final calibration. Plotted data were collected for ambient pressures and temperatures in the test chamber between $98 \mathrm{kPa}$ and $103 \mathrm{kPa}$ and $21^{\circ} \mathrm{C}$ and

$25^{\circ} \mathrm{C}$. The thermal enclosure temperature was fixed at $20.1^{\circ} \mathrm{C}$ and data were acquired using the $5.79 \mathrm{~m}$ optical sensor head connected to the base station with a 5-m long, 9/125 $\mu \mathrm{m}$ fiber.

\section{B.2 Experimental Calibration Method}

The experimental calibration simply estimated methane volume mixing ratios directly from measured absorption or $2 f$ values via an empirical function derived from experiments with known volume mixing ratios. The effectiveness of this calibration method at yielding realistic methane volume mixing ratios is limited by the ambient conditions over which the calibration data are acquired, which should span the full range of temperature, pressure, and intensity variations to be encountered during subsequent measurements.

The sample calibration data shown in this section are $1 \mathrm{~s}$ measurements of actual test chamber methane-in-air VMRs between $2.0 \mathrm{ppm}_{\mathrm{v}}$ and $200 \mathrm{ppm}_{\mathrm{v}}$, collected at the $2 v_{3}$ $\mathrm{R}(3)$ methane absorption manifold. The ambient pressure and temperature in the test chamber was measured between $98 \mathrm{kPa}$ and $103 \mathrm{kPa}$ and $21^{\circ} \mathrm{C}$ and $25^{\circ} \mathrm{C}$, and the thermal enclosure temperature was fixed at $20.1^{\circ} \mathrm{C}$. Data were acquired using the $5.79 \mathrm{~m}$ optical sensor head connected to the base station with a 6-m long, 9/125 $\mu \mathrm{m}$ fiber. 


\section{B.2.3 Experimental Calibration Example}

\section{SI Technique}

Unlike the theory-based calibration, the SI experimental calibration used here depends only on the measured absorption values, $x_{\mathrm{abs}}\left(I, I_{0}\right)$ returning from the optical sensor head. The transformation of future SI absorption measurements to a methane volume mixing ratio follows:

$$
q=G x_{\mathrm{abs}}-b
$$

Figure B.5 illustrates the residual between the SI measured methane volume mixing ratio using the experimental calibration and the generated volume mixing ratio in the test chamber. As in previous plots, the dashed and dotted lines indicate 95\% prediction and confidence intervals.

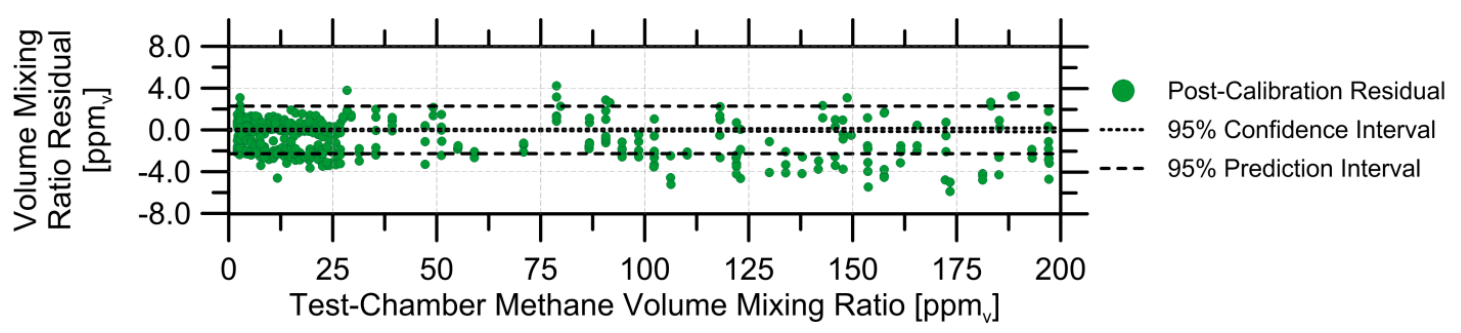

Figure B.6: Calibration residuals for WMS method 1-second averaged methane volume mixing ratio values obtained using the experimental calibration approach. Dashed lines indicate $95 \%$ prediction and confidence intervals of the final calibration function. Plotted data were collected for ambient pressures and temperatures in the test chamber between $98 \mathrm{kPa}$ and $103 \mathrm{kPa}$ and $21^{\circ} \mathrm{C}$ and $25^{\circ} \mathrm{C}$. The thermal enclosure temperature was fixed at $20.1^{\circ} \mathrm{C}$ and data were acquired using the $5.79 \mathrm{~m}$ optical sensor head connected to the base station with a 5-m long, 9/125 $\mu \mathrm{m}$ fiber.

\section{2f-WMS Technique}

The WMS experimental calibration uses an experimentally determined linear calibration factor, $G$, and zero-response, $b$ to directly related measured WMS $2 f$ values to a methane VMR.

$$
q=G x_{2 f}-b
$$


Figure B.7 illustrates the residual between the expected WMS- $2 f$ values and the results obtained post-experimental calibration, and includes the residual $95 \%$ confidence and prediction intervals. The data show a clear trend of increasing scatter in the residual as methane volume mixing ratio increases.

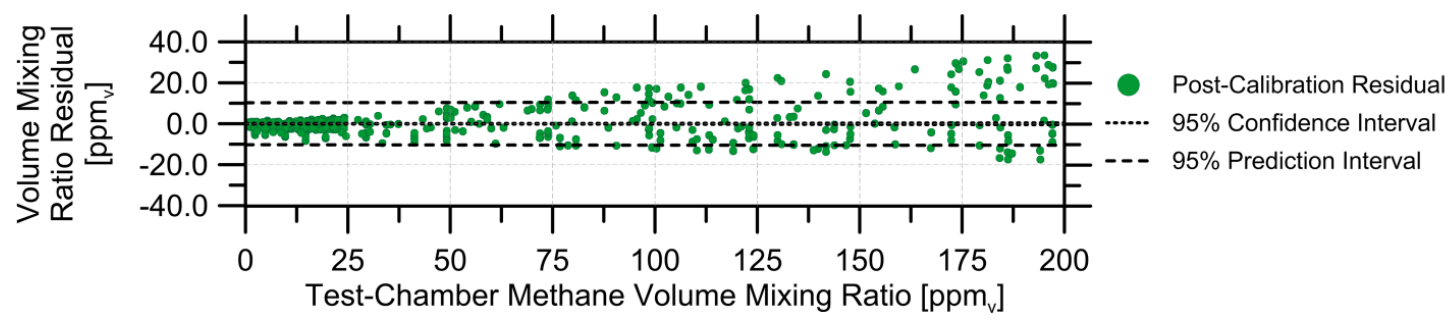

Figure B.7: Calibration residuals for WMS method 1-second averaged methane volume mixing ratio values obtained using the experimental calibration approach. Dashed lines indicate $95 \%$ prediction and confidence intervals of the final calibration function. Plotted data were collected for ambient pressures and temperatures in the test chamber between $98 \mathrm{kPa}$ and $103 \mathrm{kPa}$ and $21^{\circ} \mathrm{C}$ and $25^{\circ} \mathrm{C}$. The thermal enclosure temperature was fixed at $20.1^{\circ} \mathrm{C}$ and data were acquired using the $5.79 \mathrm{~m}$ optical sensor head connected to the base station with a 5-m long, 9/125 $\mu \mathrm{m}$ fiber.

It was observed that measured $2 f$ values, $x_{2 f}$, were correlated with the laser return intensity, $I$. To account for this dependence, a constrained fourth-order polynomial calibration surface (with coefficients, $c_{n}$ (where $n=\{0,1, \ldots, 6,7\}$ ), was fitted to the measured $x_{2 f}$ and $I$ data, which could then be used to calculate $q$.

$$
\begin{array}{r}
q=G\left[\operatorname{CAL}_{\mathrm{WMS}}\left(x_{2 f}, I\right)\right]-b \\
=x_{2 f}\left(c_{3} I^{3}+c_{2} I^{2}+c_{1} I+c_{0}\right)+c_{4} I^{3}+c_{5} I^{2}+c_{6} I+c_{7}
\end{array}
$$

Prior to field implementation, this function would have to be modified to accommodate the full range of conditions over which measurements are intended to be performed. Figure B.8 illustrates the residual between the expected WMS $2 f$ values and the results obtained post-calibration using the intensity-compensating experimental calibration function for the same measured $2 f$ data presented above. The measured mean return intensity used in this calibration falls between $0.20 \mathrm{~V}$ and $0.24 \mathrm{~V}$. 


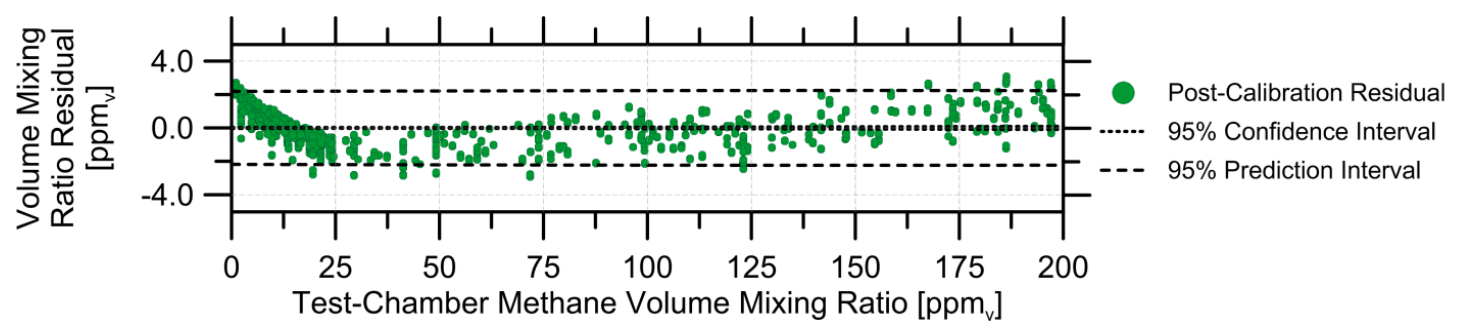

Figure B.8: Calibration residuals for WMS method 1-second averaged methane volume mixing ratio values obtained using the intensity-compensating experimental calibration approach. Dashed lines indicate $95 \%$ prediction and confidence intervals of the final calibration function. Plotted data were collected for ambient pressures and temperatures in the test chamber between $98 \mathrm{kPa}$ and $103 \mathrm{kPa}$ and $21^{\circ} \mathrm{C}$ and $25^{\circ} \mathrm{C}$. The thermal enclosure temperature was fixed at $20.1^{\circ} \mathrm{C}$ and data were acquired using the $5.79 \mathrm{~m}$ optical sensor head connected to the base station with a 5-m long, 9/125 $\mu \mathrm{m}$ fiber.

Compared to the WMS experimental calibration without return intensity compensation, the intensity-compensating experimental calibration more closely fits to the measured data as indicated by the $95 \%$ confidence interval and reduced scatter on the residual. In the thesis, use of the phrase WMS "experimental calibration" refers to the intensity-corrected calibration, unless otherwise noted. Performance of the TDLAS system when implementing WMS experimental calibration, with and without return intensity variation compensation, is discussed further in Chapter 5. 


\section{Appendix C: Precision and LDL}

The examples shown in this appendix are directly applicable to data obtained with both the SI and WMS techniques, and any of the calibration methods presented in Appendix B. This appendix provides the equations used to calculate the methane volume mixing ratio (VMR) measurement precision and lower detection limit (LDL) used to quantify system measurement performance. Both were derived from the $95 \%$ prediction intervals of a linear weighted least squares fit to a calibration dataset.

The measured methane VMR, $q$, has a variance, $s_{q}^{2}$, which is a function of the actual VMR in the test chamber during calibration, $q^{*}$. Figure C.1 shows an example plot of the variance of 1-s averaged VMR values obtained using the $2 f$-WMS method implementing a theory-based calibration. Each data point shows the variance of values obtained at test-chamber methane-in-air volume mixing ratios between 1 and $200 \mathrm{ppm}_{\mathrm{v}}$, at ambient conditions, and with a $6 \mathrm{~m}$ optical fiber coupling the $5.79 \mathrm{~m}$ optical sensor head to the base system. In this example, the measured variance reaches a maximum of $0.69 \mathrm{ppm}_{\mathrm{v}}$ at a volume mixing ratio of $200 \mathrm{ppm}_{\mathrm{v}}$ methane in air, and a minimum of $0.22 \mathrm{ppm}_{\mathrm{v}}$ at $40 \mathrm{ppm}_{\mathrm{v}}$. 


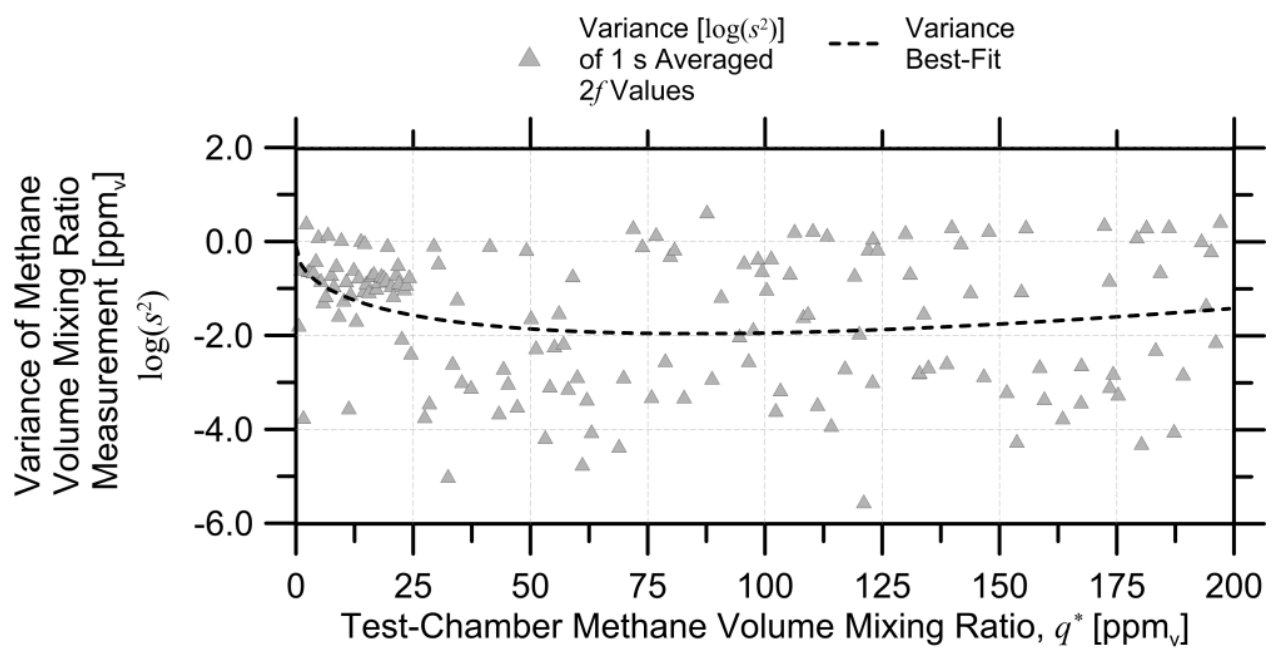

Figure C.1: Measured variance of 1-second averaged values obtained at test-chamber methane-in-air volume mixing ratios between 1 and $200 \mathrm{ppm}_{\mathrm{v}}$, with a polynomial best-fit. Data were acquired using the $2 v_{3} \mathbf{R}(3)$ methane manifold with test chamber pressures between $98 \mathrm{kPa}$ and $103 \mathrm{kPa}$, temperatures between $21^{\circ} \mathrm{C}$ and $25^{\circ} \mathrm{C}$, and with the $5.79 \mathrm{~m}$ optical sensor head coupled to the base station with a 6-m 9/125 $\mu \mathrm{m}$ fiber optic cable.

A third-order polynomial was used to describe the change in variance with $q^{*}$, following:

$$
s^{2}\left(q^{*}\right)=a_{0}+a_{1} \sqrt{q^{*}}+a_{2} q^{*}
$$

where $a_{0}, a_{1}$, and $a_{2}$ are the fit coefficients. The variance function was then used to weight the least squares fit of the linear calibration function to establish the influence of each 1-s average measurement on the final function. The weighting function follows:

$$
w\left(q^{*}\right)=\frac{1}{s^{2}\left(q^{*}\right)}
$$

\section{C.1 Prediction Interval Calculation}

The prediction intervals, $\hat{q}_{\mathrm{PI}}$, and confidence intervals, $\hat{q}_{\mathrm{CI}}$, were computed using the "Prediction Interval.vi" and "Confidence Interval.vi" functions built-in to LabView 2011, version 11.0 (LabView, 2009). The intervals were computed for a linear least-squares fit with the Student $\mathrm{t}$-factor, $t_{v ; 1-\frac{\alpha}{2}}$, with degrees of freedom, $v$ (with a value greater than 
100 for all of the calibration datasets), and probability interval, $\alpha(0.05$, corresponding to a two-sided $95 \%$ interval), following:

$$
\begin{gathered}
\hat{q}_{\mathrm{PI}}\left(q_{j}^{*}\right)=\hat{q}\left(q_{j}^{*}\right) \pm t_{v, 1-\frac{\alpha}{2}} \cdot \sqrt{\frac{\chi^{2}}{w_{j}(n-2)}+\Gamma_{j}} \\
\hat{q}_{\mathrm{CI}}\left(q_{j}^{*}\right)=\hat{q}\left(q_{j}^{*}\right) \pm t_{v, 1-\frac{\alpha}{2}} \cdot \sqrt{\Gamma_{j}}
\end{gathered}
$$

where $\chi$ is the residual between the calibration dataset (comprised of $n 1$-second methane VMR measurements, $q$ ) and the best-fit calibration function with a calibration factor, $G$, and zero-response, $b$ :

$$
\chi^{2}=\sum_{i=1}^{n} w_{i}\left[q_{i}-\left(G q_{i}^{*}+b\right)\right]^{2}
$$

and, $\Gamma_{j}$ is the $j^{t h}$ term of the diagonal of $X C X^{T}$ (written here as $\operatorname{diag}_{j}\left(X C X^{T}\right)$ ), where $X$ is:

$$
X=\left[\begin{array}{cc}
q_{1}^{*} & 1 \\
q_{2}^{*} & 1 \\
\vdots & \vdots \\
q_{n}^{*} & 1
\end{array}\right]
$$

and $C$ is the covariance matrix of the linear calibration function terms $G$ and $b$, following:

$$
C=\frac{\chi^{2}}{(n-2)}\left(X^{T} W X\right)^{-1}
$$

and $W$ is a matrix with weights for each measurement, $w_{i}$, on the diagonal:

$$
W=\left[\begin{array}{cccc}
w_{1} & 0 & \cdots & 0 \\
0 & w_{2} & \ddots & \vdots \\
\vdots & \ddots & \ddots & 0 \\
0 & \cdots & 0 & w_{n}
\end{array}\right]
$$


Expanding $X^{T} W X$

$$
\begin{aligned}
& X^{T} W X=\left[\begin{array}{cccc}
q_{1}^{*} & q_{2}^{*} & \cdots & q_{n}^{*} \\
1 & 1 & \cdots & 1
\end{array}\right]\left[\begin{array}{cccc}
w_{1} & 0 & \cdots & 0 \\
0 & w_{2} & \ddots & \vdots \\
\vdots & \ddots & \ddots & 0 \\
0 & \cdots & 0 & w_{n}
\end{array}\right]\left[\begin{array}{cc}
q_{1}^{*} & 1 \\
q_{2}^{*} & 1 \\
\vdots & \vdots \\
q_{n}^{*} & 1
\end{array}\right] \\
& =\left[\begin{array}{ll}
\sum_{i=1}^{n} w_{i} q_{i}^{* 2} & \sum_{i=1}^{n} w_{i} q_{i}^{*} \\
\sum_{i=1}^{n} w_{i} q_{i}^{*} & \sum_{i=1}^{n} w_{i}
\end{array}\right]
\end{aligned}
$$

permits the expansion of $C$ :

$$
C=\frac{\chi^{2}}{(n-2)} \frac{1}{\sum_{i=1}^{n} w_{i} q_{i}^{* 2} \sum_{i=1}^{n} w_{i}-\left(\sum_{i=1}^{n} w_{i} q_{i}^{*}\right)^{2}}\left[\begin{array}{cc}
\sum_{i=1}^{n} w_{i} & -\sum_{i=1}^{n} w_{i} q_{i}^{*} \\
-\sum_{i=1}^{n} w_{i} q_{i}^{*} & \sum_{i=1}^{n} w_{i} q_{i}^{* 2}
\end{array}\right]
$$

Finally, combining these, $\Gamma_{j}=\operatorname{diag}_{j}\left(X C X^{T}\right)$ can be expanded to:

$$
\begin{aligned}
\Gamma_{j}=\operatorname{diag}_{j}\left(X C X^{T}\right) & \\
& =\frac{1}{\sum_{i=1}^{n} w_{i} q_{i}^{* 2} \sum_{i=1}^{n} w_{i}-\left(\sum_{i=1}^{n} w_{i} q_{i}^{*}\right)^{2}}\left[\sum _ { i = 1 } ^ { n } ( q _ { i } ^ { * } - q _ { j } ^ { * } ) \left(w_{i} q_{i}^{*}\right.\right. \\
& \left.\left.-w_{i} q_{j}^{*}\right)\right]
\end{aligned}
$$

\section{C.2 Precision}

For this system, methane volume mixing ratio measurement precision was described as the maximum value of the absolute $95 \%$ prediction intervals, $\hat{q}_{\mathrm{PI}}$, over a test-chamber methane VMR range. To confirm that the measured data were normally distributed and to qualitatively verify the precision uncertainty calculated for a calibration dataset, a long-run dataset (termed "long-run dataset") comprised of at least 750 one-second measurements at constant test chamber volume mixing ratios of $\sim 2,4.5,9.0,24.0$, and $48.5 \mathrm{ppm}_{\mathrm{v}}$ methane in air was acquired using the WMS method. Histograms of the 1-s 
averaged $2 f$ values $^{5}$ were overlaid on a Gaussian distribution to qualitatively confirm a normal data distribution, and the 2.5 and 97.5 percentiles were then converted to methane volume mixing ratios (using the theory-based calibration method) to enable comparison to the prediction interval from the calibration dataset for each requested methane VMR in Figure C.2.

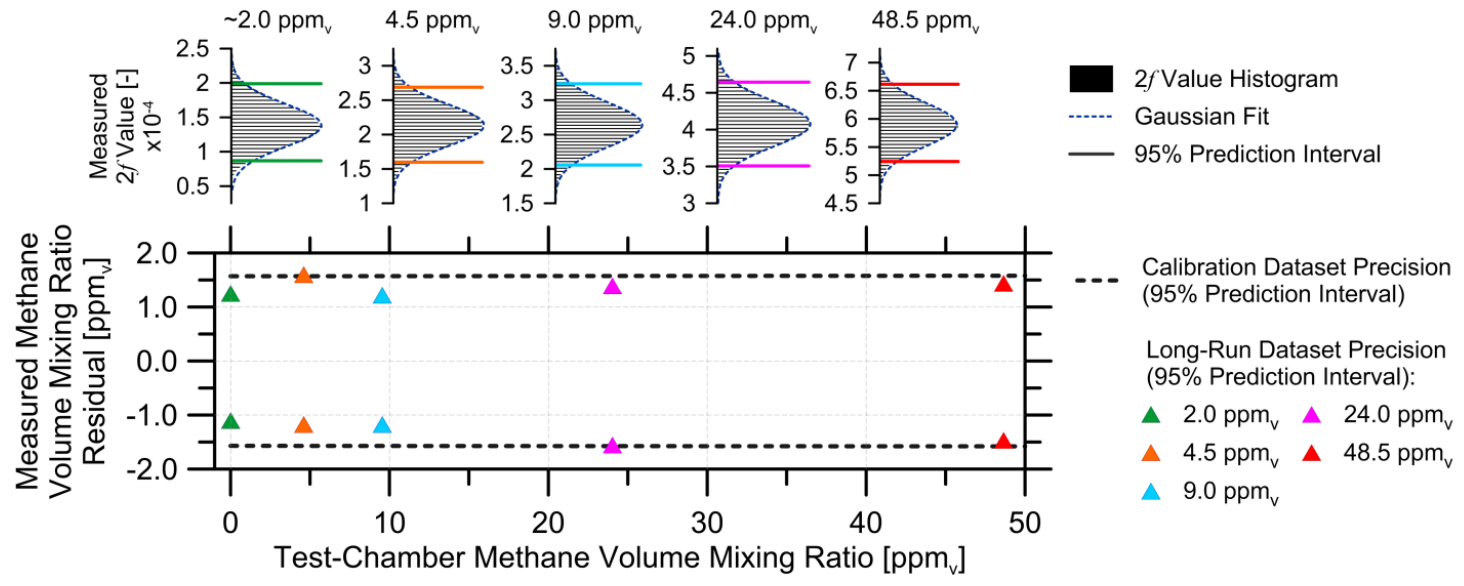

Figure C.2: Comparison of precision as determined by the $95 \%$ prediction interval of methane VMR measurements averaged for $1 \mathrm{~s}$ at test chamber methane volume mixing ratios between 2 and $50 \mathrm{ppm}_{v}$, and long-run $2.5 \%$ and $97.5 \%$ percentiles at constant test chamber volume mixing ratios of $0,4.5,9.0,24.0$, and $48.5 \mathrm{ppm}_{\mathrm{v}}$ methane in air. Data were acquired using the $2 v_{3} R(3)$ methane manifold with test chamber pressures between $98 \mathrm{kPa}$ and $103 \mathrm{kPa}$, temperatures between $21^{\circ} \mathrm{C}$ and $25^{\circ} \mathrm{C}$, and with the $5.79 \mathrm{~m}$ optical sensor head coupled to the base station with a 6-m 9/125 $\mu \mathrm{m}$ fiber optic cable.

The measured methane $2 f$ value histograms are Gaussian. The calculated prediction intervals for the calibration dataset were similar to the prediction intervals obtained from the long-run dataset. The small discrepancy could, in part, be due to the use of a single MFC for the collection of the long-run experimental data (using the 0.5 SLPM MFC, to achieve test chamber methane volume mixing ratios between 2 and $50 \mathrm{ppm}_{\mathrm{v}}$ ) whereas the calibration dataset used two configurations (0.5 SLPM MFC for ranges between 2 and $25 \mathrm{ppm}_{\mathrm{v}}$, and 2.0 SLPM MFC for ranges between 25 and $200 \mathrm{ppm}_{\mathrm{V}}$ ). As discussed in Section A.2, the uncertainties and measurement deviation tended to be higher for the 2.0 SLPM methane MFC.

\footnotetext{
${ }^{5}$ The number of bins within the histogram were based on the square root of the number of data points in the sample set
} 


\section{C.3 Lower Detection Limit}

The lower detection limit (LDL) represents the volume mixing ratio at which a feature is no longer definable above the noise floor. In spectroscopy, the widely-used (and simple) form of the LDL is defined as the VMR that generates absorption or $2 f$ values greater than three times the measurement precision. However, this method is not used here, instead the lower detection limit (LDL) can be more robustly determined following Werle et al. (Werle et al., 2004), and the limit of detection (LOD) can be computed following Loock and Wentzell (Loock and Wentzell, 2012).

The LDL is calculated based on the prediction interval, $\hat{q}_{\mathrm{PI}}$, of the calibration function, $\hat{q}$, at $95 \%$ confidence. The LDL is defined such that the lower bound of the prediction interval at the LDL is equal to the upper bound of the prediction interval at a requested volume mixing ratio of $0 \mathrm{ppm}_{\mathrm{v}}$, as illustrated in Figure C.3. The LOD is computed in a similar fashion using a $95 \%$ confidence interval, $\hat{q}_{\mathrm{CI}}$.

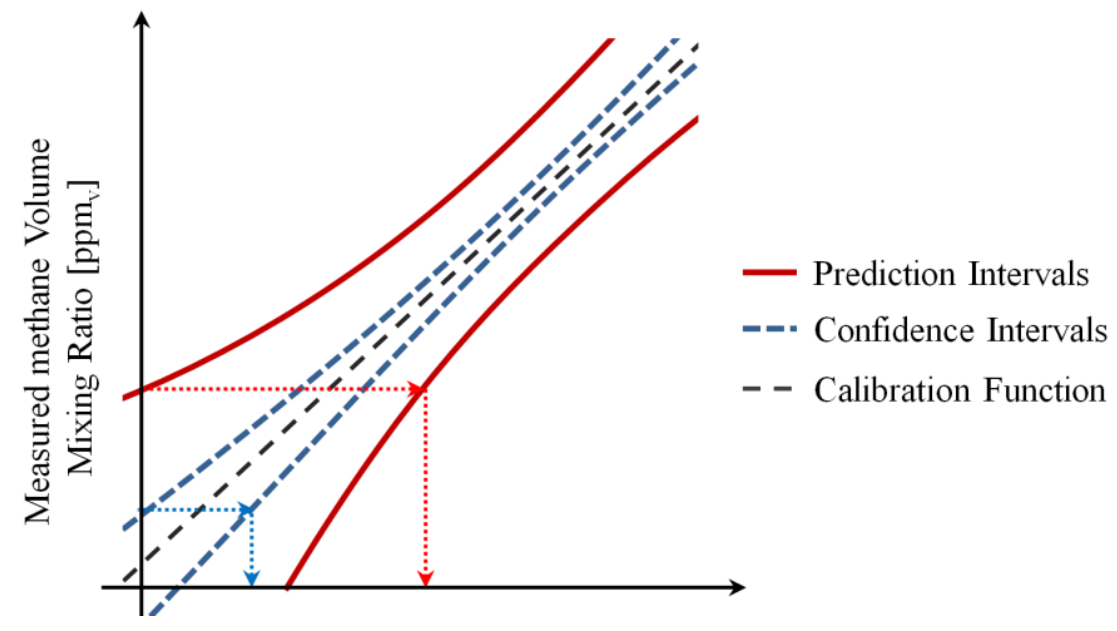

Figure C.3: Schematic illustrating the Lower Detection Limit (LDL) and Limit of Detection (LOD) using the prediction and confidence intervals of a linear calibration function 